\title{
The two-dimensional Coulomb plasma: quasi-free approximation and central limit theorem
}

\author{
Roland Bauerschmidt $^{1} \quad$ Paul Bourgade $^{2} \quad$ Miika Nikula $^{3} \quad$ Horng-Tzer Yau $^{4}$
}

\begin{abstract}
For the two-dimensional one-component Coulomb plasma, we derive an asymptotic expansion of the free energy up to order $N$, the number of particles of the gas, with an effective error bound $N^{1-\kappa}$ for some constant $\kappa>0$. This expansion is based on approximating the Coulomb gas by a quasi-free Yukawa gas. Further, we prove that the fluctuations of the linear statistics are given by a Gaussian free field at any positive temperature. Our proof of this central limit theorem uses a loop equation for the Coulomb gas, the free energy asymptotics, and rigidity bounds on the local density fluctuations of the Coulomb gas, which we obtained in a previous paper.
\end{abstract}

1 Introduction and main results

2 Preliminaries 6

3 Free energy of the torus 12

4 Proof of Theorem 1.1; quasi-free approximation of free energy 26

5 Proof of Proposition 4.5: free energy upper bound 3

6 Decoupling estimate 40

7 Proof of Proposition 4.6: free energy lower bound 51

8 Proof of Theorem 1.2 central limit theorem 59

Appendix A Rigidity estimates for Yukawa gas on torus 7

\begin{tabular}{lll} 
Appendix B Local law for the Yukawa and Coulomb gas & 81 \\
\hline
\end{tabular}

\begin{tabular}{ll} 
Notation index & 97 \\
\hline
\end{tabular}

\begin{tabular}{ll} 
References & $\mathbf{9 9}$ \\
\hline
\end{tabular}

\section{Introduction and main results}

1.1. One-component plasma. The two-dimensional one-component Coulomb plasma (OCP) is a Gibbs measure on the configurations of $N$ charges $\mathbf{z}=\left(z_{1}, \ldots, z_{N}\right) \in \mathbb{C}^{N}$. Given an external potential $V: \mathbb{C} \rightarrow \mathbb{R} \cup\{+\infty\}$, the Hamiltonian of this measure is defined by

$$
H_{N, V}^{G}(\mathbf{z})=N \sum_{j} V\left(z_{j}\right)+\sum_{j \neq k} G\left(z_{j}, z_{k}\right)
$$

\footnotetext{
${ }^{1}$ University of Cambridge, Statistical Laboratory, DPMMS, rb812@cam.ac.uk.

${ }^{2}$ New York University, partially supported by NSF grant DMS-1513587, bourgade@cims.nyu.edu .

${ }^{3}$ Harvard University, Center of Mathematical Sciences and Applications, minikula@cmsa.fas.harvard.edu.

${ }^{4}$ Harvard University, Department of Mathematics, partially supported by NSF grant DMS-1606305, 1855509 and a Simons Investigator award, htyau@math.harvard.edu.
} 
where $G\left(z_{j}, z_{k}\right)=\mathcal{C}\left(z_{j}-z_{k}\right)$ is the two-dimensional Coulomb potential,

$$
\mathcal{C}\left(z_{j}-z_{k}\right)=-\log \left|z_{j}-z_{k}\right|
$$

characterized by $\Delta \log |\cdot|=2 \pi \delta_{0}$ as distributions and $\sum_{i \neq j}=2 \sum_{i<j}$. The Coulomb plasma is our main interest, but throughout the paper we will also consider other symmetric interactions $G\left(z_{j}, z_{k}\right)$. The associated canonical Gibbs measure at the inverse temperature $\beta>0$ is defined by

$$
P_{N, V, \beta}^{G}(\mathrm{~d} \mathbf{z})=\frac{1}{Z_{N, V, \beta}^{G}} \mathrm{e}^{-\beta H_{N, V}^{G}(\mathbf{z})} m^{\otimes N}(\mathrm{~d} \mathbf{z}),
$$

where $m$ denotes the Lebesgue measure on $\mathbb{C}$, and $Z_{N, V, \beta}^{G}$ the normalization constant. Here we have assumed that $V$ has sufficient growth at infinity, so that the latter is well-defined. We will follow the convention that when $G=\mathcal{C}$ then we will omit the superscript $\mathcal{C}$ whenever there is no confusion. Similar conventions apply to other subscripts, i.e., we will often omit $N$ and $\beta$.

Throughout the paper, we will use the terms Coulomb plasma, Coulomb gas, and OCP to refer to the measure $P_{N, V, \beta}$. This model has connections with a variety of models in mathematical physics and probability theory. For $\beta=1$, it describes the eigenvalues density for some measures on non-Hermitian random matrices [16,22]. In particular, for quadratic $V$ the complex vector $\mathbf{z}$ is distributed like the spectrum of a matrix with complex Gaussian entries. Moreover, the properties of this two-dimensional gas are known to be related to the fractional quantum Hall effect: for $\beta=2 s+1$, with $s$ integer, $P_{N, V, \beta}$ is the density obtained from Laughlin's guess for wave functions of fractional fillings of type $(2 s+1)^{-1}[31$. Finally, an important problem is the crystallization of the two-dimensional Coulomb gas for small temperature [2,15].

The Coulomb plasma is a system with two scales: the microscopic scale describing distances comparable to the typical interparticle distance $N^{-1 / 2}$ and the macroscopic scale describing distances of order 1. At the macroscopic scale, the empirical particle measure concentrates around a limiting density that is described by classical potential theory, which we now describe. For potentials $V$ that are lower semicontinuous and satisfy the growth condition

$$
\liminf _{|z| \rightarrow \infty}(V(z)-(2+\varepsilon) \log |z|)>-\infty
$$

for some $\varepsilon>0$, it is well known (see e.g. 43) that there exists a compactly supported equilibrium measure $\mu_{V}$ that is the unique minimizer of the convex energy functional

$$
\mathcal{I}_{V}(\mu)=\iint \log \frac{1}{|z-w|} \mu(\mathrm{d} z) \mu(\mathrm{d} w)+\int V(z) \mu(\mathrm{d} z)
$$

over the set of probability measures on $\mathbb{C}$. The unique minimizer $\mu_{V}$ is supported on a compact set $S_{V}$ and, assuming that $V$ is smooth, it has the density

$$
\rho_{V}=\frac{1}{4 \pi} \Delta V \mathbb{1}_{S_{V}}
$$

with respect to the Lebesgue measure $m$. We write $I_{V}=\mathcal{I}_{V}\left(\mu_{V}\right)$ for the minimum of $\mathcal{I}_{V}$. For $\mathbf{z} \in \mathbb{C}^{N}$, the empirical measure is defined by

$$
\hat{\mu}=\hat{\mu}^{\mathbf{z}}=\frac{1}{N} \sum_{j} \delta_{z_{j}} .
$$

For arbitrary $\beta \in(0, \infty)$, it is well-known that $\hat{\mu} \rightarrow \mu_{V}$ vaguely in probability as $N \rightarrow \infty$, with $\hat{\mu}$ distributed under $P_{N, V, \beta}$. In [6] (see also 32 ) we have proved two stronger estimates for the 
Coulomb gas, which in can be summarized as follows. For $b>0$ and $k \in \mathbb{N}$, we introduce the norms

$$
\|f\|_{\infty, k, b}=\sum_{j=0}^{k} b^{j}\left\|\nabla^{j} f\right\|_{\infty}, \quad\|f\|_{\infty, k}=\|f\|_{\infty, k, 1} .
$$

Note that the boundedness of $\|f\|_{\infty, k, b}$ means that $f$ is smooth at scale $b$. We typically take $b=N^{-s}$ for some $s \in[0,1 / 2)$, and assume that $f$ is supported in a disk of radius of order $b$. The first estimate proved in [6] is a local law that asserts that for any smooth $f$ supported in a disk of radius $b=N^{-s}(s \in[0,1 / 2))$ centered at some point $z_{0}$ in the bulk (i.e., interior) of $S_{V}$ (and the function $f$ supported in the bulk when $s=0$ ), we have

$$
\begin{aligned}
\frac{1}{N} \sum_{j=1}^{N} f\left(z_{j}\right)-\int f(z) \mu_{V}(\mathrm{~d} z) & =\mathrm{O}(\log N)\left(N^{-1-2 s}\|\Delta f\|_{\infty}+N^{-\frac{1}{2}-s}\|\nabla f\|_{L^{2}}\right) \\
& =\mathrm{O}(\log N) N^{-1 / 2-s}\|f\|_{\infty, 2, N^{-s}},
\end{aligned}
$$

where $\|f\|_{L^{2}}=\left(\int|f|^{2} \mathrm{~d} m\right)^{1 / 2}$ is the $L^{2}$-norm of $f$, with very high probability. A stronger estimate, which we shall call rigidity, asserting that

$$
\sum_{j=1}^{N} f\left(z_{j}\right)-N \int f(z) \mu_{V}(\mathrm{~d} z)=\mathrm{O}\left(N^{\varepsilon}\right)\|f\|_{\infty, 4, N^{-s}}
$$

with very high probability, also holds under the same assumptions.

The main result of this paper is the identification of the random error term in the above rigidity estimate. It is given by the Gaussian free field with a nonzero mean.

1.2. Main results. Our main results are the following two theorems. In addition to the condition (1.4), the global potential $V$ is always assumed to satisfy

$V \in \mathscr{C}^{5}$ on a neighborhood of $S_{V}=\operatorname{supp} \mu_{V}, \quad \alpha_{0} \leqslant \Delta V(z) \leqslant \alpha_{0}^{-1}$ for all $z \in S_{V}$

for some constant $\alpha_{0}>0$. We assume that the boundary of $S_{V}$ is piecewise $\mathscr{C}^{1}$, or more precisely that $\partial S_{V}$ is a finite union of $\mathscr{C}^{1}$ curves. The prototypical example is $V(z)=|z|^{2}$ in which case $S_{V}$ is a disk, and the convergence $\hat{\mu} \rightarrow \mu_{V}$ is known as the circular law in random matrix theory.

Theorem 1.1. There exists a constant $\zeta_{\beta}^{\mathcal{C}} \in \mathbb{R}$ such that, for any external potential $V$ satisfying the conditions (1.4) and (1.10), for any $\kappa<1 / 24$,

$$
\frac{1}{\beta N} \log \int \mathrm{e}^{-\beta H_{V}} m^{\otimes N}(\mathrm{~d} \mathbf{z})=-N I_{V}+\frac{1}{2} \log N+\zeta_{\beta}^{\mathcal{C}}+\left(\frac{1}{2}-\frac{1}{\beta}\right) \int \rho_{V} \log \rho_{V} \mathrm{~d} m+\mathrm{O}\left(N^{-\kappa}\right) .
$$

A similar result, as a limiting statement instead of a quantitative error bound, and with $\zeta_{\beta}^{\mathcal{C}}$ characterized via a large deviation principle, was previously proved in 33. For our application to the proof of Theorem 1.2 below, a quantitative error bound is essential. In addition, we will provide a physical interpretation of $\zeta_{\beta}^{\mathcal{C}}$ as the residual free energy of the Coulomb (or technically a long-range Yukawa) gas on the torus; see Theorems 3.1 and 4.1 .

For the statement of Theorem 1.2 , we require the following additional definitions. For any function $f$ with support in $S_{V}$, let

$$
\begin{aligned}
& X_{V}^{f}=\sum_{j} f\left(z_{j}\right)-N \int f \mathrm{~d} \mu_{V} \\
& Y_{V}^{f}=\frac{1}{4 \pi} \int \Delta f \log \Delta V \mathrm{~d} m=\frac{1}{4 \pi} \int \Delta f(z) \log \rho_{V}(z) m(\mathrm{~d} z) .
\end{aligned}
$$


In the following theorem, $f: \mathbb{C} \rightarrow \mathbb{R}$ is supported on a disk with radius $b=N^{-s}$ for a fixed scale $s \in[0,1 / 2)$, and $\|f\|_{\infty, 5, b} \leqslant C<\infty$ uniformly in $N$. We also assume that the support of $f$ satisfies $\operatorname{dist}\left(\operatorname{supp}(f), S_{V}^{c}\right)>\varepsilon$ for some $\varepsilon>0$ uniformly in $N$. (Indeed, the last condition can be relaxed to $\varepsilon=N^{-1 / 4+c}$ for arbitrarily small $c$, i.e., $f$ still supported in the bulk).

Theorem 1.2. Suppose that $V$ satisfies the condition (1.4) and 1.10 , and that $f$ has support in a ball of radius $b=N^{-s}$ with the above conditions. Then there exists $\tau_{0}=\tau_{0}(s)>0$ such that for any $0<\tau<\tau_{0}$ and $0<\lambda \ll\left(N b^{2}\right)^{1-2 \tau}$, we have

$$
\frac{1}{\beta \lambda} \log \mathbb{E}\left(\mathrm{e}^{-\beta \lambda\left(X_{V}^{f}-\left(\frac{1}{\beta}-\frac{1}{2}\right) Y_{V}^{f}\right)}\right)=\frac{\lambda}{8 \pi} \int|\nabla f(z)|^{2} m(\mathrm{~d} z)+\mathrm{O}\left(\left(N b^{2}\right)^{-\tau}\right) .
$$

Here the expectation is with respect to $P_{N, V, \beta}^{\mathcal{C}}$.

Note that $\lambda$ is allowed to be very large in this theorem; this provides strong error estimates for the Gaussian convergence. This central limit theorem is noteworthy due to the absence of normalization: fluctuations of $X_{V}^{f}$ are only of order one, due to repulsion, but still Gaussian. For the purpose of establishing the central limit theorem for $X_{V}^{f}$, it suffices to take $\lambda$ to be of order one (independent of $N$ ).

Finally, a result similar to Theorem 1.2 was obtained simultaneously and independently in 34 .

1.3. Related results. The study of one- and two-dimensional Coulomb and log-gases has attracted considerable attention recently, see e.g. 21 for many aspects of these probability measures in connection with statistical physics. The subject of our work, abnormally small Gaussian charge fluctuations of the one-component plasma, was first predicted in the late 1970s (see [26] and the references therein).

In dimension two, in the special case $\beta=1$, the central limit theorem was first proved for the Ginibre ensemble, i.e. for quadratic external potential $V$ 39, 40. These results were extended to more general $V$ by combining tools from determinantal point processes and the loop equation approach [4,5]. In particular, in the latter works the determinantal structure was used to prove local isotropy of the point process, an important a priori estimate necessary to the loop equation approach. For general inverse temperature $\beta$, the determinantal structure does not hold; nevertheless an expansion of the partition function and correlation functions was predicted in [48 50]. The expansion of the partition function up to order $N$ was rigorously obtained in 33 (along with a corresponding large deviation principle for a tagged point process); see also the related earlier works 41, 44, 45]; in addition, see also [23]. Still for the two-dimensional Coulomb gas at any temperature, a local density $6,3,32,36]$ was recently proved, together with abnormally small charge fluctuations in the sense of rigidity [6], see [1.9). Other recent results in this direction include $3,37,38,42]$.

For the log-gas on the line, much more is known. Indeed, in dimension one the Selberg integrals are often a good starting point to evaluate partition functions, and anisotropy does not cause any trouble in the analysis of loop equations. For general $\beta$ and $V$, full expansions of the partition function and correlators were predicted in [19], proved at first orders in [46] and at all orders in [8,9]. A natural analogue of the rigidity (1.9) is also known to hold for log-gases on the real line 10. Still for the log-gas in dimension 1, the central limit theorem was first discovered on the circle for $\beta=2$ in [27, and on the real line for any $\beta$ in 28. For test functions supported on a mesoscopic scale, the local central limit theorem was proved on the circle for some compact groups in [4], for general $\beta$ ensembles with quadratic $V$ in [11] and for general $V$ in [7]. 
For expansions at high temperatures, and exponential decay of microscopic correlations, in closely related models of Coulomb gases, see [13,25]. For results on crystallization in the onedimensional one-component Coulomb plasma, see [1, 12,30]. Further results on Coulomb systems in statistical mechanics are reviewed in [14,21].

1.4. Proof sketch. In Section 3, we first prove that an extended version of Theorem 1.1 holds for Yukawa gases on a torus. The essence is to show that the constant $\zeta_{\beta}^{\mathcal{C}}$, to be called the residual free energy, can be identified independently of the range of the Yukawa interaction. This fact is then used in Section 4 to establish an expansion of the free energy of the Coulomb gas up to order $N^{1-\kappa}$. The main idea is to approximate the Coulomb gas first by a short-range Yukawa gas, and then by a quasi-free Yukawa gas. Roughly speaking, a Yukawa gas with range $\ell \ll 1$ can be viewed, for the purpose of computing free energy, as an ideal gas consisting of independent squares of size $b$ satisfying $1 \gg b \gg \ell$ and with the gas inside each square being a periodic Yukawa gas with range $\ell$. Since this gas is an ideal gas over a distance longer than a mesoscopic scale $b$, we call it a quasi-free approximation.

The Yukawa approximation to the Coulomb gas is a well-known tool in the study of the quantum Coulomb gas, see, e.g., 17,18. However, the precision needed here is far beyond the previous results. Following the traditional approaches in free energy estimates, we will prove the free energy expansion of the Coulomb gas by establishing a lower and an upper bound. The proof of the upper bound, contained in Section 5, consists of the standard argument of counting two-body Yukawa interactions in neighboring squares and uses only that the density of Coulomb gas is bounded for all scales $\geq N^{-1 / 2+\varepsilon}$ by [6]. The lower bound turns out to be much more difficult than the upper bound. The Yukawa gas used in the approximation of the Coulomb gas is constructed from the Yukawa gas on periodic squares, so the resulting Yukawa gas on the plane breaks the translational and rotational invariances of the Coulomb gas. The translational invariance is easy to restore by averaging over the "grid" of the squares. The rotational invariance, however, is hard to recover and the effects of breaking it has to be estimated precisely. We remark that in the quantum Coulomb gas, the lower bound of the free energy was proved [35] by carefully maintaining the Coulomb rotational invariance. This was possible due to the use of the "Swiss cheese" approximation. In our setting, we are forced to maintain the square approximation since the limits of the residual free energy were established only for squares. The key estimate which allows us to control the breaking of the rotational invariance is contained in Section 6, where we estimate the energy distortion resulting from embedding torus into the Euclidean space. This estimate uses the rigidity estimate of the periodic Yukawa gas, a parallel version of the rigidity estimate established in 6 for the Coulomb gas. Using the estimates from Section 6, we complete the proof of the lower bound of the free energy in Section 7 .

Another difficulty in establishing Theorem 1.1 is the surface energy of a Coulomb gas. The typical inter-particle distance of this gas is $N^{-1 / 2}$, therefore the total Coulomb energy for particles within a distance $N^{-1 / 2}$ to the boundary of the support of the equilibrium measure is of order $N$. To see this, note that the number of surface particles, i.e., particles with distance of order $N^{-1 / 2}$ to the boundary, is of order $\sqrt{N}$. Thus their Coulomb interaction energy is of order $N$. Theorem 1.1 requires to capture these interaction energies up to order $N^{1-\kappa}$. In other words, the leading term in the energy associated with the charges near the boundary of the support of the Coulomb gas has to be identified. Our idea is to use an ideal gas approximation for a boundary layer and then switch to a Yukawa approximation for interior particles. We will explain this idea in Section 4 .

In Section 8, we first prove that the central limit theorem holds after subtracting a random 
term, the local angle term. From this result and the asymptotic expansion of the free energy for the Coulomb gas, Theorem 1.1, we obtain that the angle term does in fact vanish in a large deviation sense. We thus prove Theorem 1.2 for a test function $f$ with macroscopic support. For test functions with support on a mesoscopic scale $b$, we proceed via conditioning to a disk of radius $2 b$. This conditioning procedure was used in [6]; it has the advantage of reformulating the question into a problem on the natural scale $b$.

Throughout the paper, we will extensively use the local density and rigidity estimates for the Yukawa gas and Coulomb gas with additional angular interaction, in a form similar to (1.8) and (1.9). In Appendices $\mathrm{A}, \mathrm{B}$, we therefore extend the estimates of $[6]$ to the Yukawa gas and the Coulomb gas with angle term. The rigidity estimates, to be proved in Appendix A, use estimates of the local laws in Appendix B. We reverse the logic order because the proofs of the local laws in Appendix $\mathrm{B}$ are technical and use extensively conventions from 6 .

1.5. Notation. We use the usual Landau $\mathrm{O}$ and o symbols. For $N$-dependent quantities $A, B \geqslant$ 0 , we write $A \ll B$ when there exists $\varepsilon>0$ and $N_{0} \geqslant 0$ such that $A \leqslant N^{-\varepsilon} B$ for $N \geqslant N_{0}$. For an event $E$, we say that $E$ holds with high probability if for all $D>0$ there is $N_{D}$ such that $\mathbb{P}(E) \geqslant 1-N^{-D}$ for $N \geqslant N_{D}$. For random variables $A$ and $B$, we write $A \prec B$ if for any $\varepsilon>0$ the event $|A| \leqslant N^{\varepsilon}|B|$ holds with high probability. We use the notation $A=\mathrm{O}\left(N^{-\infty}\right)$ to denote that $A$ is subpolynomially small: for every $D>0$ one has $|A| \leqslant N^{-D}$ for all $N \geqslant N_{D}$ with probability at least $1-N^{-D}$ (if $A$ is a random variable).

\section{Preliminaries}

We begin with the definitions of the Coulomb and Yukawa gas ensembles, and we give a summary of the potential theory that we require, as well as of the estimates on the local density.

2.1. Coulomb and Yukawa potentials. We will identify $\mathbb{R}^{2}$ and $\mathbb{C}$ and usually write $z$ and $w$ for its elements. The two-dimensional Coulomb potential is $C(z)=-\log |z|$, satisfying $-\Delta C=2 \pi \delta_{0}$ as distributions. The Yukawa potential with range $\ell>0$ is the solution to $\left(-\Delta+1 / \ell^{2}\right) Y^{\ell}=2 \pi \delta_{0}$. Explicitly, the two-dimensional Yukawa potential is given by the formula

$$
Y^{\ell}(z):=\frac{1}{4 \pi} \int_{\mathbb{R}^{2}} \mathrm{e}^{-\mathrm{i} p \cdot z} \int_{0}^{\infty} \mathrm{e}^{-t\left(p^{2}+1 / \ell^{2}\right) / 2} \mathrm{~d} t \mathrm{~d} p=\int_{1}^{\infty} \mathrm{e}^{-a(s+1 / s)} \frac{\mathrm{d} s}{s}=: g(a), \quad a=\frac{|z|}{2 \ell},
$$

where $p \cdot z$ denotes the Euclidean inner product on $\mathbb{R}^{2}$. From this formula, note that $Y^{\ell}(z)$ is pointwise positive and positive definite, and that there is an absolute constant $Y_{0}$ such that

$$
Y^{\ell}(z) \begin{cases}\sim-\log |z|+\log \ell+Y_{0}+\mathrm{O}(|z| / \ell) & \text { if }|z| / \ell \leqslant 1 \\ \leq C_{1} \mathrm{e}^{-C_{2}|z| / \ell} & \text { if }|z| / \ell \geq 1 .\end{cases}
$$

Indeed, the asymptotic relation can be checked with constant $Y_{0}=\log 2+\vartheta$ from

$$
g(a)=\vartheta-\log a+\mathrm{O}(a), \quad \vartheta=\int_{0}^{\infty}\left(\mathrm{e}^{-s}-1_{s<1}\right) \frac{\mathrm{d} s}{s},
$$

where $g$ was defined in (2.1). In particular, up to the constant $Y_{0}+\log \ell$, the two-dimensional Coulomb potential $-\log |z|$ is the limit $\ell \rightarrow \infty$ of $Y^{\ell}(z)$. We denote by $\mathbb{T}$ the two-dimensional unit torus $\mathbb{C} / \mathbb{Z}^{2}$. For $\ell>0$, the Yukawa interaction of range $\ell$ on $\mathbb{T}$ is defined by

$$
U^{\ell}(z)=\sum_{n \in \mathbb{Z}^{2}} Y^{\ell}(z+n)
$$


2.2. Ensembles. We now define the Coulomb gas and its perturbed versions on the plane $\mathbb{C}$, and the Yukawa gas on the torus $\mathbb{T}$.

Coulomb (and Yukawa) gas on the plane. Remember that for a one-particle potential $V: \mathbb{C} \rightarrow$ $\mathbb{R} \cup\{+\infty\}$ and the two-particle interaction $G: \mathbb{C} \times \mathbb{C} \backslash \triangle \rightarrow \mathbb{R}$ on $\mathbb{C}$, where $\triangle=\left\{(z, w) \in \mathbb{C}^{2}\right.$ : $z=w\}$, we define the $N$-particle Hamiltonian by

$$
H_{N, V}^{G}(\mathbf{z})=N \sum_{j} V\left(z_{j}\right)+\sum_{j \neq k} G\left(z_{j}, z_{k}\right), \quad\left(\mathbf{z} \in \mathbb{C}^{N}\right)
$$

and the corresponding Gibbs measures at inverse temperature $\beta>0$ by

$$
P_{N, V, \beta}^{G}(\mathrm{~d} \mathbf{z})=\frac{1}{Z_{N, V, \beta}^{G}} \mathrm{e}^{-\beta H_{N, V}^{G}(\mathbf{z})} m^{\otimes N}(\mathrm{~d} \mathbf{z}),
$$

where $Z_{N, V, \beta}^{G}$ is the partition function. The Coulomb interaction is obtained by taking $G(z, w)=$ $\mathcal{C}(z-w)$ to be the Coulomb potential and we omit the argument $G$ in that case; the Yukawa interaction of range $\ell$ is obtained with $G(z, w)=Y^{\ell}(z-w)$ and we then write $\ell$ instead of $Y^{\ell}$ in the superscript. For the Coulomb case, we sometimes use the convention $\ell=\infty$.

On the plane, we only use the Yukawa potential as a regularization of the Coulomb potential, with $\ell \geqslant N^{2}$, in which case it is for all of our purposes equivalent to a Coulomb potential.

Yukawa gas on the torus. Similarly, for $\ell>0$ and for a potential $V: \mathbb{T} \rightarrow \mathbb{R}$, the $N$-particle Hamiltonian of the periodic Yukawa gas on $\mathbb{T}$ is defined by

$$
H_{N, V}^{\ell}(\mathbf{z})=N \sum_{j} V\left(z_{j}\right)+\sum_{j \neq k} U^{\ell}\left(z_{j}-z_{k}\right), \quad\left(\mathbf{z} \in \mathbb{T}^{N}\right)
$$

where $U^{\ell}$ was defined in (2.4) and we here use the abbreviation $H_{N}^{\ell}=H_{N, 0}^{\ell}$ The corresponding probability measures are again defined as in (2.6), with $m$ now the Lebesgue measure on $\mathbb{T}$.

On the torus, we use the Yukawa potential with short range compared to the side length of the torus (but still large with respect to the interparticle spacing), i.e., $N^{-1 / 2} \ll \ell \ll 1$.

Perturbed Coulomb gas on the plane. We will also consider perturbations of the Coulomb gas on the plane, for which the two-particle interaction takes the form

$$
G(z, w)=\mathcal{C}(z-w)+t \tilde{G}(z, w)
$$

with $t \in \mathbb{R}$, and where we assume that the perturbation $\tilde{G}$ satisfies, for some $\theta>0$,

$$
|\tilde{G}(z, w)| \leqslant 1, \quad|\tilde{G}(z, w)| \leqslant \mathrm{e}^{-|z-w|^{2} /\left(2 \theta^{2}\right)} .
$$

The perturbed Coulomb gas will be used only in Section 8. We therefore suggest the reader to skip this material until it is used in Section 8 .

2.3. Potential theory. We define variational functionals for the Yukawa potential with external potential $V$ on probability measures $\mu$ on $\mathbb{C}$ by

$$
\mathcal{I}_{V}^{\ell}(\mu)=\int V(z) \mu(\mathrm{d} z)+\int Y^{\ell}(z-w) \mu(\mathrm{d} z) \mu(\mathrm{d} w) .
$$

In the definition of the variational functional for the Coulomb interaction, the Yukawa potential $Y^{\ell}$ is replaced by the Coulomb potential $C$, and we then again omit the superscript $\ell$. Moreover, we use the analogous definition for the variational functional of the Yukawa gas on the torus $\mathbb{T}$, where $Y^{\ell}$ is replaced by $U^{\ell}$. We always make the following assumptions: 
(i) The set $\Sigma_{V}=\{z: V(z)<\infty\}$ has positive logarithmic capacity; see [43, Section I.1].

(ii) The potential $V$ is locally in $C^{1,1}$ and, for the full plane, it satisfies the growth condition

$$
\liminf _{|z| \rightarrow \infty}(V(z)-\varepsilon \log |z|)>-\infty
$$

In the Yukawa case, we assume $\varepsilon>0$, whereas in the Coulomb gas we assume that $\varepsilon>2$. In the case of the torus, the growth assumption is trivial.

For a probability measure $\mu$ on $\mathbb{C}$ respectively $\mathbb{T}$, define the Yukawa potential by

$$
Y_{\mu}^{\ell}(z)=\int Y^{\ell}(z-w) \mu(\mathrm{d} w), \quad \text { respectively } \quad U_{\mu}^{\ell}(z)=\int U^{\ell}(z-w) \mu(\mathrm{d} w),
$$

and again we use analogous notation in the Coulomb case. The following standard result gives the existence and uniqueness of the equilibrium measure for the Yukawa and Coulomb gas. Let $P\left(\Sigma_{V}\right)$ be the set of probability measures supported in $\Sigma_{V}$. We write $m=1 / \ell$ and use the convention $m^{2}=0$ for the Coulomb case.

Theorem 2.1. Consider the Yukawa potential of range $\ell$ on $\mathbb{C}$ or the Coulomb potential on $\mathbb{C}$ (with the convention $\ell=\infty$ ). Suppose satisfies assumption (i)-(ii) above. Then there exists a unique $\mu_{V}^{\ell} \in P\left(\Sigma_{V}\right)$ such that

$$
\mathcal{I}_{V}^{\ell}\left(\mu_{V}^{\ell}\right)=\inf \left\{\mathcal{I}_{V}^{\ell}(\mu): \mu \in P\left(\Sigma_{V}\right)\right\}
$$

The support $S_{V}^{\ell}=\operatorname{supp} \mu_{V}^{\ell}$ is bounded (uniformly in $\ell$ ) and of positive capacity, and $\mathcal{I}_{V}^{\ell}\left(\mu_{V}^{\ell}\right)<$ $\infty$. Furthermore, the energy-minimizing measure $\mu_{V}^{\ell}$ may be characterized as the unique element $\mu$ of $P\left(\Sigma_{V}\right)$ for which there exists a constant $c_{V} \in \mathbb{R}$ such that Euler-Lagrange equation

$$
\begin{array}{ll}
Y_{\mu}^{\ell}+\frac{1}{2} V=c_{V} & \text { q.e. in } S_{V}^{\ell} \quad \text { and } \\
Y_{\mu}^{\ell}+\frac{1}{2} V \geqslant c_{V} & \text { q.e. in } \mathbb{C}
\end{array}
$$

holds. The equilibrium measure $\mu_{V}^{\ell}$ in the set $S_{V}^{\ell}$ is given by

$$
\mu_{V}^{\ell}=\frac{1}{4 \pi}\left(\Delta V+m^{2}\left(2 c_{V}-V\right)\right)=\frac{1}{4 \pi}\left(\left(\Delta-m^{2}\right) V+2 m^{2} c_{V}\right)
$$

where the Laplacian is understood in the distributional sense. The same statement holds for the Yukawa potential on the torus $\mathbb{T}$ with $Y_{\mu}^{\ell}$ replaced by $U_{\mu}^{\ell}$.

The proof is identical to that of the Coulomb case; see e.g. 43. Also, by the same argument, under the assumption that $V$ satisfies (1.4), the support of $\mu_{V}^{\ell}$ is compact uniformly in $\ell$.

In the case of the Yukawa gas on the torus with $V=0$, by translation-invariance, the unique minimizer in 2.12 is the uniform probability measure on $\mathbb{T}$. Hence the minimum energy of the variational functional for the Yukawa gas on the unit torus is simply given by

$$
\inf _{\mu \in P(\mathbb{T})} \int U^{\ell}(z-w) \mu(\mathrm{d} z) \mu(\mathrm{d} w)=2 \pi \ell^{2} .
$$

We will use this fact in Section 3 . 
2.4. Local density estimates. From now on, we always assume that $V$ satisfies the assumptions of Theorem 2.1. The local density estimates stated in the following theorems imply that, for any disk $B$ of radius $r \gg N^{-1 / 2}$ (and the respective support assumptions), the number of particles in $B$ is of order $r^{2}$ with high probability under the respective ensemble. For their statements, given a test function $f: \mathbb{C} \rightarrow \mathbb{R}$, we denote the linear statistic centered by the equilibrium measure by

$$
X_{f}=\sum f\left(z_{k}\right)-N \int f(z) \mu_{V}^{\ell}(\mathrm{d} z)=N \int f(z) \tilde{\mu}(\mathrm{d} z)
$$

where $\hat{\mu}=\frac{1}{N} \sum_{j} \delta_{z_{j}}$ denotes the empirical measure, and

$$
\tilde{\mu}=\hat{\mu}-\mu_{V}^{\ell}
$$

The following two theorems will be proved in the Appendix B.

Theorem 2.2 (Local density for the torus). Consider the Yukawa gas on the torus $\mathbb{T}$ with Hamiltonian (2.7) and assume the potential $V$ satisfies (i) above and $\operatorname{supp}\left(\mu_{V}\right)=\mathbb{T}$. For any $f: \mathbb{T} \rightarrow \mathbb{R}$ supported in a disk of radius $b \gg N^{-1 / 2}$,

$$
\left|X_{f}\right| \prec \sqrt{N b^{2}\left(f,\left(-\Delta+m^{2}\right) f\right)}+b^{2}\|\Delta f\|_{\infty} .
$$

In particular, for any disk $B \subset \mathbb{T}$ with radius $b \gg N^{-1 / 2}$, with high probability, we have

$$
N \hat{\mu}(B)=\mathrm{O}\left(N b^{2}\right)
$$

Theorem 2.3 (Local density estimate on the plane). Suppose that $V$ satisfies the conditions (1.4) and (1.10). Consider either the Coulomb gas on $\mathbb{C}$ with potential $V$ and Hamiltonian (2.5), the perturbed Coulomb gas in $(2.9)$ with $|t| \theta^{2} N \leqslant 1$, or the Yukawa gas with range $\ell \geqslant N^{2}$. Then for any $f: \mathbb{C} \rightarrow \mathbb{R}$ supported in a disk of radius $b \gg N^{-1 / 2}$ that is contained in $S_{V}$ and has a distance $\gg N^{-1 / 4}+t^{1 / 4}$ to $\partial S_{V}$,

$$
\left|X_{f}\right| \prec \sqrt{N b^{2}(f,-\Delta f)}+b^{2}\left\|\nabla^{2} f\right\|_{\infty}=\mathrm{O}\left(\sqrt{N b^{2}}\right)\|f\|_{\infty, 2, b} .
$$

In particular, for any disk $B \subset S_{V}$ with radius $b \gg N^{-1 / 2}$ and distance $\gg N^{-1 / 4}+t^{1 / 4}$ to $\partial S_{V}$, with high probability,

$$
N \hat{\mu}(B)=\mathrm{O}\left(N b^{2}\right) \text {. }
$$

Moreover, if $D=\left\{z \in S_{V}: \operatorname{dist}\left(z, \partial S_{V}\right) \leqslant b^{\prime}\right\}$ with $b^{\prime} \gg N^{-1 / 4}$ then, with high probability,

$$
N \hat{\mu}(D)=\mathrm{O}\left(N b^{\prime}\right)
$$

2.5. Rigidity estimates. In addition to the local density estimates of the previous subsection, for the Yukawa gas on the torus, we also need the stronger rigidity estimates given by the following theorems. These theorems are proved in Appendix A, again following the method of [6].

Theorem 2.4 (Rigidity estimate for Yukawa gas on the torus). Consider the Yukawa gas on the unit torus of range $\ell$. Let $s \in\left(0, \frac{1}{2}\right)$, and assume that $N^{-1 / 2} \ll \ell \ll 1$ and that $V=0$. For any sufficiently smooth $f: \mathbb{T} \rightarrow \mathbb{R}$ supported in a ball of radius $b=N^{-s}$,

$$
\left|X_{f}\right| \prec\left(\frac{b}{\ell}+1\right)^{2}\|f\|_{\infty, 3, b} .
$$


In the regime that $b / \ell \leq 1$, this estimate improves the previous local density estimate by about a factor $1 /(\sqrt{N} b)$ with a price of taking one more derivative in the test function $f$.

As a corollary, we obtain the following proposition which estimates functions of two points. The proposition, proved in Appendix $\mathrm{A}$, is a direct application of the rigidity estimate just stated and Taylor expansion. To state the estimate, for any sufficiently smooth function $g: \mathbb{T} \times \mathbb{T} \rightarrow \mathbb{R}$, we denote

$$
g_{\mathrm{B}_{t}}^{(j)}(z, w)=\sup _{(x, y) \in \mathrm{B}_{t}(z) \times \mathrm{B}_{t}(w)}\left|\nabla^{j} g(x, y)\right|,
$$

where $\mathrm{B}_{t}(z)$ is the Euclidean ball of radius $t$ centered at $z$ and $\left|\nabla^{j} g(x, y)\right|$ is the maximum over all partial derivatives of $g$ of order $j$.

Proposition 2.5. Consider the Yukawa gas on the unit torus of range $\ell$. Assume that $N^{-1 / 2} \ll$ $\ell \ll 1$ and that $V=0$. Fix $N^{-1 / 2} \ll s \ll 1$. Then for any smooth function $g$ on $\mathbb{T} \times \mathbb{T}$ and any fixed $p \in \mathbb{N}$,

$$
N^{2} \iint g(z, w) \tilde{\mu}(\mathrm{d} z) \tilde{\mu}(\mathrm{d} w) \prec\left(\frac{1}{s^{4}}+\frac{1}{\ell^{4}}\right) \sum_{j=0}^{p-1} s^{j}\left\|\nabla^{j} g\right\|_{1} N^{2} s^{p}\left\|g_{\mathrm{B}_{s}}^{(p)}\right\|_{1}
$$

where $\|\cdot\|_{1}$ is the $L^{1}$-norm on $\mathbb{T} \times \mathbb{T}$ and $\left\|\nabla^{j} g\right\|_{1}=\left\|\mid \nabla^{j} g\right\|_{1}$.

Notice that, besides explicit factors, $t$ only appears in the error term $g_{\mathrm{B}_{t}}^{(p)}$. We usually choose $t$ to be slightly smaller than the scale that the function $g$ is smooth on.

2.6. Conditioned local density estimates. To prove the mesoscopic versions of the central limit theorem, in addition to the above local density estimates, we need conditioned versions of these. These and can be skipped on the first reading.

To state the conditioned estimates, we first recall the local conditioning from [6, Section 5]. We first focus on the Coulomb gas on the plane and comment on the changes for the Yukawa gas on the torus afterwards. Let $B \subset \mathbb{C}$ be a disk of radius $b$ contained in $S_{V}$, and consider the Coulomb gas obtained by conditioning on all of the particles outside $B$. More precisely, for a particle configuration $z \in \mathbb{C}^{N}$, let $M=M(z)$ denote the number of particles in $B$, let $\left(\tilde{z}_{1}, \tilde{z}_{2}, \ldots, \tilde{z}_{M}\right)$ denote the collection of particles inside $B$, and let $\left(\hat{z}_{1}, \hat{z}_{2}, \ldots, \hat{z}_{N-M}\right)$ denote the particles outside $B$. The Hamiltonian $H_{N, V}$ may then be written as

$$
H_{N, V}(z)=\sum_{j \neq k} \log \frac{1}{\left|\tilde{z}_{j}-\tilde{z}_{k}\right|}+N \sum_{j}\left(V\left(\tilde{z}_{j}\right)-V_{o}\left(\tilde{z}_{j} \mid \hat{z}\right)\right)+E(\hat{z})
$$

where

$$
V_{o}(w \mid \hat{z})=-\frac{2}{N} \sum_{k} \log \frac{1}{\left|w-\hat{z}_{k}\right|}, \quad E(\hat{z})=\sum_{j \neq k} \log \frac{1}{\left|\hat{z}_{j}-\hat{z}_{k}\right|}+N \sum_{j} V\left(\hat{z}_{j}\right) .
$$

The term $E(\hat{z})$ is independent of the particles in $B$ and is thus irrelevant for the conditioned measure. For any configuration of external particles $\hat{z} \in(\mathbb{C} \backslash B)^{N-M}$ and $z \in \mathbb{C}$, we write

$$
\begin{aligned}
W(w \mid \hat{z}) & = \begin{cases}\frac{N}{M}\left(V(w)-V_{o}(w \mid \hat{z})\right) & (w \in B), \\
+\infty & (w \notin B),\end{cases} \\
P_{N, V, \beta}(\mathrm{d} w \mid \hat{z}) & =P_{M(\hat{z}), W(\cdot \mid \hat{z}), \beta}(\mathrm{d} w) .
\end{aligned}
$$


The Coulomb gas given by the potential $W(\cdot \mid \hat{z})$ is the conditional gas inside $B$, given the external configuration $\hat{z}$. Here we have used the convention of the measure $P_{N, V, \beta}(\mathrm{d} w \mid \hat{z})$ in $(1.3)$; this convention also explains the normalization factor $N / M$ in (2.28). In [6], it was proven that under our assumptions on $V$ the conditional potential satisfies the following properties. First, since $V_{o}(\cdot \mid \hat{z})$ is harmonic in $B$ we have

$$
\mu_{W}=\frac{\Delta W(z)}{4 \pi}=\frac{N}{M} \mu_{V}
$$

in the interior of the support $S_{W} \subset B$ (where $\mu_{W}$ and its support $S_{W}$ are defined by minimization of the Coulomb version of $(2.10)$ ). For any function $f$ that has compact support in $S_{W}$, we thus have

$$
M \int f \mathrm{~d} \mu_{W}=N \int f \mathrm{~d} \mu_{V}
$$

Finally, from 6 , Sections 5-6], we know that the measure $\mathrm{d} \mu_{W}$ may be expressed as $\frac{N}{M} \mathbb{1}_{S_{W}} \mathrm{~d} \mu_{V}+$ $v \mathrm{~d} s$, where $\mathrm{d} s$ is the length measure on $\partial B, v \in L^{\infty}(\partial B)$, and that the following properties hold. These properties are verified in the proof of [6, Theorem 6.1].

The same definitions and properties apply in the Yukawa case when the Coulomb potential is replaced by the Yukawa potential (the analogues of [6, Sections 5-6] are proved in Appendix B], when (2.30) is replaced by the Yukawa density of the form (2.14), and when (2.31) is restricted to test functions with $\int f \mathrm{~d} m=0$.

Lemma 2.6. Consider the perturbed Coulomb gas on the plane as in Theorem 2.3 or the Yukawa gas on the torus as in Theorem 2.2. For any $s \in\left(0, \frac{1}{2}\right)$, there exists a constant $\tau>0$ such that the following statements hold with probability at least $1-\mathrm{e}^{-N r^{2}}$ for $r=N^{-s}$ :

$$
\begin{aligned}
& M=N \mu_{V}(B)\left(1+\mathrm{O}\left(M^{-\tau}\right)\right), \\
& S_{W} \supset\left\{z \in B: d(z, \partial B)>M^{-\tau} r\right\} \\
& \mu_{W}(\partial B)=\int v \mathrm{~d} s \leqslant M^{-\tau}, \\
& \|v\|_{\infty} \leqslant \mathrm{O}(1 / r) .
\end{aligned}
$$

In particular, any disk $B$ in the lemma satisfies the following good boundary conditions:

Definition 2.7 (Good boundary conditions). Fix a scale $N^{-1 / 2+\varepsilon} \leq r \ll 1$. Let $B$ be a disk of radius $r$, let $P(\cdot \mid \hat{z})$ be the conditional law (with the particles $\hat{z}$ outside $B$ fixed) of the Coulomb gas induced on $z \in B^{M(\hat{z})}$ where $M(\hat{z})$ is the number of particles contained in $B$, and let $W(\cdot \mid \hat{z})$ be the corresponding potential (with $W(\cdot \mid \hat{z})=+\infty$ outside $B$ ). We say that the boundary condition $\hat{z}$ of the conditional law are good boundary conditions if the following properties hold. The equilibrium measure associated to $W=W(\cdot \mid \hat{z})$ of the conditional measure can be decomposed as $\mu_{W}(\mathrm{~d} z)=\rho_{W}(z) m(\mathrm{~d} z)+v(z) \mathrm{d} s$, where $\mathrm{d} s$ is the length measure on $\partial S_{W}$ and $S_{W} \subset B$. Furthermore, there exists a disk $\Omega$ of radius $r\left(1-N^{-\tau}\right)$ for some $\tau>0$ such that the equilibrium measure satisfies the bounds

$$
\sum_{k=0}^{3} r^{k}\left\|\left(\nabla^{k} \rho_{W}\right) 1_{\Omega}\right\|_{\infty} \leqslant K, \quad \frac{1}{\left|S_{W}\right|} \int_{\Omega} \rho_{W}(z) m(\mathrm{~d} z) \geqslant 1-M^{-a}, \quad\|v\|_{\infty} \leqslant M^{A}
$$

for some constants $a>0, A \geqslant 0, K>0$. 
Theorem 2.8. In the setting of Theorem 2.2, let $B$ be a disk of radius $r$ with good boundary conditions, and write $n=N r^{2}$. Then good boundary conditions in the sense of Definition 2.7 hold with high probability under the original measure. Furthermore, for any disk $B^{\prime} \subset S_{W}$ with radius $N^{-1 / 2} \ll b \ll r$ and distance er to $\partial S_{W}$, with high probability under the conditioned measure, the conditioned version of (2.18) holds (where $X_{f}$ is defined with respect to $V$ ):

$$
\left|X_{f}\right| \prec \sqrt{N b^{2}\left(f,\left(-\Delta+m^{2}\right) f\right)}+b^{2}\|\Delta f\|_{\infty} .
$$

Theorem 2.9. In the setting of Theorem 2.3, let $B$ be a disk of radius $r$ with good boundary conditions, and write $n=N r^{2}$ and $t=N^{-2 \sigma}$. Then good boundary conditions in the sense of Definition 2.7 hold with high probability under the original measure. Furthermore, for any disk $B^{\prime} \subset S_{W}$ with radius $N^{-1 / 2} \ll b \ll r$ and distance $\gg\left(n^{-1 / 4}+n^{-\sigma / 2}\right) r$ to $\partial S_{W}$, with high probability under the conditioned measure, the conditioned version of (2.20) holds:

$$
\left|X_{f}\right| \prec \sqrt{N b^{2}(f,-\Delta f)}+b^{2}\left\|\nabla^{2} f\right\|_{\infty} .
$$

\section{$3 \quad$ Free energy of the torus}

We start with proving a version of Theorem 1.1 for the Yukawa gas on the torus. This outlines the strategy for the proof of Theorem 1.1 in a simplified context and also constructs the constant $\zeta$ in Theorem 1.1 .

3.1. Main result. Recall the definition of the Yukawa gas on the unit torus from Section 2.2 and also that the minimum energy of the variational functional for the Yukawa gas on the unit torus is given by $2 \pi \ell^{2}$ by (2.15). We denote the $N$-particle partition function of the Yukawa gas on the unit torus with range $\ell$ by

$$
Z_{N}^{(\ell)}=\int_{\mathbb{T}^{N}} \mathrm{e}^{-\beta H_{N}^{\ell}(\mathbf{z})} m(\mathrm{~d} \mathbf{z})
$$

where $H_{N}^{\ell}$ was defined in 2.7). The main result of this section is the following theorem, namely a version of Theorem 1.1 for the Yukawa gas on the torus.

Theorem 3.1. There exists a $\beta$ dependent constant $\zeta$, the residual free energy of the Yukawa gas on the torus, such that for any $\sigma>0$ there is $\kappa>0$ such that if $N^{-1 / 2+\sigma} \leqslant \ell \ll 1$,

$$
\frac{1}{\beta} \log Z_{N}^{(\ell)}=-2 \pi \ell^{2} N^{2}+N \log \ell+\frac{1}{2} N \log N+N \zeta+\mathrm{O}\left(N^{1-\kappa}\right) .
$$

More precisely, $\mathrm{O}\left(N^{1-\kappa}\right)$ is $N^{\varepsilon} \mathrm{O}\left(N^{7 / 8}+N^{1-2 \sigma}\right)$.

Remark 3.2. The above statement holds without the assumption $\ell \ll 1$. Since this generalization is not needed for our application, we restrict to this slightly simplified case.

To prove Theorem 3.1, we define the specific residual free energy in a system of $N$ particles with interaction range $\ell$ by

$$
\zeta^{(\ell)}(N)=\frac{1}{N} \xi^{(\ell)}(N)-\frac{1}{2} \log N, \quad \xi^{(\ell)}(N)=\frac{1}{\beta} \log Z_{N}^{(\ell)}+2 \pi \ell^{2} N^{2}-N \log \ell .
$$

In this notation, Theorem 3.1 asserts that $\zeta^{\ell}(N)=\zeta+\mathrm{O}\left(N^{1-\kappa}\right)$ whenever $\ell \geqslant N^{-1 / 2+\sigma}$.

Along this section and in Section 4 , we will repeatedly use the Jensen inequality in the form

$$
\log \int \mathrm{e}^{-B}+\mathbb{E}^{B}(B-A) \leq \log \int \mathrm{e}^{-A} \leq \log \int \mathrm{e}^{-B}+\mathbb{E}^{A}(B-A),
$$

where $\mathbb{E}^{A} X=\frac{\int \mathrm{e}^{-A} X}{\int \mathrm{e}^{-A}}$ and integration is with respect to a fixed measure. 
3.2. Continuity of the residual free energy. In the following Lemmas 3.3 and 3.5 , it is proved that $\zeta^{(\ell)}(N)$ is almost independent of the range $\ell$ provided that $\ell \gg N^{-1 / 2}$, and that $\zeta^{(\ell)}(N)$ depends only weakly on the number of particles $N$.

Lemma 3.3. For any $\sigma \in\left(0, \frac{1}{2}\right)$ and $\nu$ and $\omega$ such that $N^{-1 / 2+\sigma} \leqslant \nu \leqslant \omega \ll 1$, the following inequality holds:

$$
\mathrm{O}\left(N^{-2 \sigma+\varepsilon}\right) \leqslant \zeta^{(\omega)}(N)-\zeta^{(\nu)}(N) \leqslant \mathrm{O}\left(N^{-\infty}\right),
$$

where the notation $\mathrm{O}\left(N^{-\infty}\right)$ was defined at the end of Section 1 .

Proof. We start with the upper bound on $\zeta^{(\omega)}-\zeta^{(\nu)}$. By Jensen's inequality,

$$
\frac{1}{\beta} \log \int \mathrm{e}^{-\beta H_{N}^{\omega}(\mathbf{z})} m(\mathrm{~d} \mathbf{z}) \leq \frac{1}{\beta} \log \int \mathrm{e}^{-\beta H_{N}^{\nu}(\mathbf{z})} m(\mathrm{~d} \mathbf{z})-\mathbb{E}^{H_{N}^{\omega}}\left[H_{N}^{\omega}-H_{N}^{\nu}\right] .
$$

Let $L_{\omega}^{\nu}(z)=U^{\omega}(z)-U^{\nu}(z)$. Then $L_{\omega}^{\nu}(0)=\log (\omega / \nu)+\mathrm{O}\left(N^{-\infty}\right)$ since $U^{\ell}(0)=Y^{\ell}(0)+O\left(e^{-c / \ell}\right)$ and $\nu \leqslant \omega \ll 1$. Since $L_{\omega}^{\nu}$ is positive definite, as can be verified by representing it in Fourier space, we also have

$$
\mathbf{L}_{\omega}^{\nu}=\int L_{\omega}^{\nu}(z-w) \tilde{\mu}(\mathrm{d} w) \tilde{\mu}(\mathrm{d} z) \geqslant 0, \quad \text { for } \nu \leqslant \omega \ll 1 .
$$

Together with $\int U^{\ell}(z) m(\mathrm{~d} z)=2 \pi \ell^{2}$ by 2.15$)$, we have the estimate

$$
H_{N}^{\omega}-H_{N}^{\nu}=\sum_{j \neq k} L_{\omega}^{\nu}\left(z_{j}-z_{k}\right)=2 \pi\left(\omega^{2}-\nu^{2}\right) N^{2}-N \log (\omega / \nu)+N^{2} \mathbf{L}_{\omega}^{\nu}+\mathrm{O}\left(N^{-\infty}\right) .
$$

By the definition $(3.2)$, this proves that $\zeta^{(\omega)}(N)-\zeta^{(\nu)}(N) \leqslant \mathrm{O}\left(N^{-\infty}\right)$.

For the lower bound, we use the Jensen inequality and (3.7) to obtain

$$
\begin{aligned}
& \frac{1}{\beta} \log \int \mathrm{e}^{-\beta H_{N}^{\omega}(\mathbf{z})} m(\mathrm{~d} \mathbf{z}) \\
& \quad \geq \frac{1}{\beta} \log \int \mathrm{e}^{-\beta H_{N}^{\nu}(\mathbf{z})} m(\mathrm{~d} \mathbf{z})-2 \pi\left(\omega^{2}-\nu^{2}\right) N^{2}+N \log (\omega / \nu)-N^{2} \mathbb{E}^{H_{N}^{\nu}} \mathbf{L}_{\omega}^{\nu}+\mathrm{O}\left(N^{-\infty}\right) .
\end{aligned}
$$

We apply the two-point rigidity estimate 2.25) with $g(z, w)=L_{\omega}^{\nu}(z, w), \ell=\nu, t=\nu N^{-\varepsilon}$ and $p=2 / \varepsilon$. Note that this choice of $g$ satisfies

$$
\begin{gathered}
t^{j}\left\|\nabla^{j} g\right\|_{1} \leq C_{j} t^{j} \nu^{-j} \omega^{2} \leq C_{j} \omega^{2}, \\
t^{p}\left\|g_{\mathrm{B}_{t}}^{(p)}\right\|_{1} \leq C_{p} t^{p} \nu^{-p} \omega^{2} \leq C_{p} N^{-p \varepsilon} \omega^{2} \leq C_{p} N^{-2} .
\end{gathered}
$$

Therefore 2.25) gives

$$
N^{2} \mathbb{E}^{H_{N}^{\nu}} \mathbf{L}_{\omega}^{\nu} \leqslant N^{4 \varepsilon} \mathrm{O}\left(\omega^{2} \nu^{-4}\right)
$$

Replacing $\varepsilon$ by $\varepsilon / 4$, we have thus proved that

$$
\zeta^{(\omega)}(N)-\zeta^{(\nu)}(N) \geqslant N^{\varepsilon} \mathrm{O}\left(\omega^{2} \nu^{-4}\right) .
$$

This estimate can be improved to give the lower bound stated in (3.4) as follows. For any fixed $\varepsilon>0$ small, we choose $\left(\nu_{i}\right)_{i=1}^{k}$ such that $\nu_{1}=\omega, \nu_{k}=\nu$ and $1 \leqslant \nu_{j} / \nu_{j+1} \leqslant N^{\varepsilon}$. Since $\omega \leqslant 1$ by assumption, there exists an admissible choice of $k$ depending on $\varepsilon$ but not on $N$. Then (3.10) with $(\omega, \nu)$ replaced by $\left(\nu_{j}, \nu_{j+1}\right)$ gives

$$
\zeta^{(\omega)}(N)-\zeta^{(\nu)}(N)=\sum_{j=1}^{k-1}\left(\zeta^{\left(\nu_{j}\right)}(N)-\zeta^{\left(\nu_{j+1}\right)}(N)\right)=\sum_{j=1}^{k-1} N^{\varepsilon} \mathrm{O}\left(\nu_{j}^{2} \nu_{j+1}^{-4}\right)=N^{\varepsilon} \mathrm{O}\left(\nu^{-2}\right) .
$$

Since $\nu^{-2} \leqslant N^{1-2 \sigma}$ by assumption, this completes the proof of the lemma. 
We record the following rough bound on the partition function.

Lemma 3.4. The torus residual free energy satisfies

$$
\xi^{(\gamma)}(n)=\mathrm{O}(n \log n)
$$

Proof. This bound follows by smearing out the point charges into charge densities and using the positive definiteness for the upper bound and Jensen's inequality for the lower bound. This is a standard argument and therefore we omit the details. The interested reader can look into [6, Proposition 4.1] or B.46.

Using the above bound on the partition function, we obtain the following estimate for its dependence on $n$.

Lemma 3.5. The torus residual free energy satisfies

$$
\zeta^{(\gamma)}(n)-\zeta^{(\gamma)}(m)=\mathrm{O}\left(|m-n| \frac{\log (n+m)}{n+m}\right) .
$$

Proof. We now prove the following more precise version of (3.12):

$$
\xi^{(\gamma)}(n)+2 \pi \gamma^{2}-\log \gamma \leq \xi^{(\gamma)}(n+1) \leq \xi^{(\gamma)}(n)+\mathrm{O}(\log n) .
$$

In particular, $\xi^{(\gamma)}(n+1)-\xi^{(\gamma)}(n)=\mathrm{O}(\log n)$. This implies the claim as follows. If $n \leq m \leq 2 n$, then

$$
\zeta^{(\gamma)}(m)-\zeta^{(\gamma)}(n)=\sum_{k=n}^{m} \mathrm{O}\left(\frac{\log k}{k}\right)=\mathrm{O}\left(|m-n| \frac{\log m}{n}\right)=\mathrm{O}\left(|m-n| \frac{\log (n+m)}{n+m}\right) .
$$

On the other hand, if $m \geq 2 n$, then already 3.12 implies

$$
\zeta^{(\gamma)}(m)-\zeta^{(\gamma)}(n)=\mathrm{O}(\log n)+\mathrm{O}(\log m)=\mathrm{O}\left(|m-n| \frac{\log (n+m)}{n+m}\right) .
$$

This proves the claim for $n \leqslant m$. The case $n>m$ follows by exchanging the roles of $n$ and $m$.

It remains to prove (3.14). We start with the lower bound. By Jensen's inequality,

$$
\log \frac{\int e^{-\beta \sum_{i \neq j ; i, j=1}^{n+1} U^{\gamma}\left(z_{i}-z_{j}\right)} m^{n}(\mathrm{~d} \mathbf{z})}{\int \mathrm{e}^{-\beta \sum_{i \neq j ; i, j=1}^{n} U^{\gamma}\left(z_{i}-z_{j}\right)} m^{n}(\mathrm{~d} \mathbf{z})} \geq-2 \beta \mathbb{E}_{n}^{\gamma} \sum_{j=1}^{n} U^{\gamma}\left(z_{n+1}-z_{j}\right),
$$

where $m^{n}(\mathrm{~d} \mathbf{z})=\prod_{j=1}^{n} m\left(\mathrm{~d} z_{j}\right)$. In the following, we omit the superscript whenever it is obvious. Integrating both sides over $z_{n+1}$, and again using Jensen's inequality, we get

$$
\log Z_{n+1} \geq \int m\left(\mathrm{~d} z_{n+1}\right) \log \int \mathrm{e}^{-\beta \sum_{i \neq j ; i, j=1}^{n+1} U^{\gamma}\left(z_{i}-z_{j}\right)} m(\mathrm{~d} \mathbf{z}) \geq \log Z_{n}-(2 n \beta)\left(2 \pi \gamma^{2}\right) .
$$

By the definition of $\xi^{(\gamma)}(n)$, it follows that

$$
\begin{aligned}
\xi^{(\gamma)}(n+1) & =2 \pi \gamma^{2}(n+1)^{2}-(n+1) \log \gamma+\frac{1}{\beta} \log Z_{n+1}(\beta) \\
& \geq 2 \pi \gamma^{2}(n+1)^{2}-2 n\left(2 \pi \gamma^{2}\right)+\frac{1}{\beta} \log Z_{n}(\beta)-(n+1) \log \gamma=\xi^{(\gamma)}(n)+2 \pi \gamma^{2}-\log \gamma
\end{aligned}
$$


This gives the lower bound in (3.14).

For the upper bound, we set $H_{k}=\sum_{i \neq j, i, j \neq k}^{n+1} U^{\gamma}\left(z_{i}-z_{j}\right)$. Then, by Hölder's inequality, it follows that

$$
Z_{n+1}(\beta)=\int \exp \left[-\frac{\beta}{n-1} \sum_{k=1}^{n+1} \hat{H}_{k}\right] m(\mathrm{~d} \mathbf{z}) \leq \int \mathrm{e}^{-\beta \frac{n+1}{n-1} \hat{H}_{k}} m(\mathrm{~d} \mathbf{z})=Z_{n}\left(\beta \frac{n+1}{n-1}\right) .
$$

Since $\xi^{(\gamma)}(n)=\mathrm{O}(n \log n)$, we have

$$
\frac{1}{\beta} \log \int \mathrm{e}^{-\beta H_{n}} m(\mathrm{~d} \mathbf{z})=-2 \pi \gamma^{2} n^{2}+\mathrm{O}(n \log n), \quad H_{n}=\sum_{i \neq j}^{n} U^{\gamma}\left(z_{i}-z_{j}\right) .
$$

Using this estimate and the convexity of the function $t \rightarrow \log \int \mathrm{e}^{-t H_{n}} m(\mathrm{~d} \mathbf{z})$, we have

$$
-\mathbb{E}_{n}^{\gamma, \beta} H_{n} \leq \log \int \mathrm{e}^{-(\beta+1) H_{n}} m(\mathrm{~d} \mathbf{z})-\log \int \mathrm{e}^{-\beta H_{n}} m(\mathrm{~d} \mathbf{z}) \leq-2 \pi \gamma^{2} n^{2}+\mathrm{O}(n \log n) .
$$

Using (3.17) and integrating the relation $\partial_{\beta} \log Z_{n}(\beta)=-\mathbb{E}_{n}^{\gamma, \beta} H_{n}$, we therefore get

$$
\begin{aligned}
\log Z_{n+1}(\beta) \leq \log Z_{n}\left(\beta \frac{n+1}{n-1}\right) & =\log Z_{n}(\beta)-\int_{\beta}^{\beta \frac{n+1}{n-1}} \mathbb{E}_{n}^{\gamma, s} H_{n} \mathrm{~d} s \\
& \leq \log Z_{n}(\beta)-2 \pi \gamma^{2} \frac{2 n^{2} \beta}{n-1}+\mathrm{O}(\log n)
\end{aligned}
$$

In summary, we have proved that

$$
\begin{aligned}
\xi^{(\gamma)}(n+1) & =2 \pi \gamma^{2}(n+1)^{2}-(n+1) \log \gamma+\frac{1}{\beta} \log Z_{n+1}(\beta) \\
& \leq 2 \pi \gamma^{2}(n+1)^{2}+\frac{1}{\beta} \log Z_{n}(\beta)-2 \pi \gamma^{2} \frac{2 n^{2}}{n-1}-n \log \gamma-\log \gamma+\mathrm{O}(\log n) \\
& =\xi^{(\gamma)}(n)+\mathrm{O}(\log n),
\end{aligned}
$$

which is the upper bound in (3.14).

3.3. Scaling relation. In the remainder of this section, we will often consider the Yukawa gas on a torus of side length $b$. Let $\mathbb{T}^{(b)}$ denote the torus of side length $b$, i.e., the square $[b / 2, b / 2)^{2}$ with horizontal respectively vertical sides identified. The Yukawa potential on $\mathbb{T}^{(b)}$, under this identification to the square $[b / 2, b / 2)^{2}$, is defined by

$$
U_{b}^{\ell}(z)=U_{b}^{(\gamma)}(z)=\sum_{n \in(b \mathbb{Z})^{2}} Y^{\ell}(z+n),
$$

where $Y^{\ell}$ is the full plane Yukawa potential defined in (2.1) and $\gamma=\ell / b$ denotes the relative interaction range from now on. We denote the corresponding partition function of the $n$-particle Yukawa gas by $Z_{b, n}^{(\gamma)}$, and set

$$
\xi_{b}^{(\gamma)}(n)=\frac{1}{\beta} \log Z_{b, n}^{(\gamma)}+2 \pi \gamma^{2} n^{2}-n \log \ell, \quad Z_{b, n}^{(\gamma)}=\int_{\left(\mathbb{T}^{(b)}\right)^{n}} \mathrm{e}^{-\beta \sum_{i \neq j} U_{b}^{\ell}\left(w_{i}-w_{j}\right)} m(\mathrm{~d} \mathbf{w}) .
$$

From now on, we adopt the following convention for $z-w$ in $\mathbb{T}^{(b)}$ including the case $b=1$. 
Definition 3.6. For $z, w \in \mathbb{T}^{(b)}$, we always choose the representative for $z-w$ (which is only defined modulo $\left.(b \mathbb{Z})^{2}\right)$ to be in $[-b / 2, b / 2)^{2}$.

For later use, we record the following scaling relation.

Lemma 3.7. For any $K>0$,

$$
\xi_{K b}^{(\gamma)}(n)=\left(\frac{1}{\beta}-\frac{1}{2}\right) n \log K^{2}+\xi_{b}^{(\gamma)}(n)
$$

In particular, by choosing $K=b^{-1}$, with the definition of $\zeta$ from (3.2),

$$
\xi_{b}^{(\gamma)}(n)=n \zeta^{(\gamma)}(n)+\frac{n}{2} \log n+n\left(\frac{1}{2}-\frac{1}{\beta}\right) \log b^{-2} .
$$

Proof. By definition of the Yukawa potential (3.18), we have $U_{K b}^{K \ell}(K r)=U_{b}^{\ell}(r)$. Therefore, by changing variables to $z=w K$,

$$
\begin{aligned}
\frac{1}{\beta} Z_{n, K b}^{(\gamma)}= & \frac{1}{\beta} \log \int_{\left(\mathbb{T}^{(K b)}\right)^{n}} \mathrm{e}^{-\beta \sum_{i \neq j}^{n} U_{K b}^{K \ell}\left(z_{i}-z_{j}\right)} m(\mathrm{~d} \mathbf{z}) \\
& =\frac{1}{\beta} \log \int_{\left(\mathbb{T}^{(b)}\right)^{n}} \mathrm{e}^{-\beta \sum_{i \neq j} U_{b}^{\ell}\left(w_{i}-w_{j}\right)} m(\mathrm{~d} \mathbf{w})+\frac{1}{\beta} n \log K^{2}=\frac{1}{\beta} \log Z_{n, b}^{(\gamma)}+\frac{1}{\beta} n \log K^{2},
\end{aligned}
$$

where the term with $\log K^{2}$ comes from the scaling factor in the Jacobian. With $\gamma=\ell / b$ and using the definition 3.19 of $\xi$, we have the rescaling identity

$$
\xi_{K b}^{(\gamma)}(n)=2 \pi \gamma^{2} n^{2}-n \log K \ell+\frac{1}{\beta} n \log K^{2}+\frac{1}{\beta} \log Z_{n, b}^{(\gamma)}=\left(\frac{1}{\beta}-\frac{1}{2}\right) n \log K^{2}+\xi_{b}^{(\gamma)}(n)
$$

as claimed.

3.4. Quasi-free approximation. To prove Theorem 3.1 we first replace the interaction range $\ell$ by $N^{-1 / 2+\sigma}$ for an arbitrary fixed $\sigma>0$. By Lemma 3.3, this replacement contributes an error $N^{\varepsilon} O\left(N^{1-2 \sigma}\right)$ to $(3.1)$. From now on, we therefore assume that $\ell=N^{-1 / 2+\sigma}$.

In the following, we always parametrize the unit torus $\mathbb{T}$ by the square $[-1 / 2,1 / 2)^{2}$. For a parameter $b \ll 1$ such that $1 / b$ and $N b^{2}$ are both integers, we then divide the unit torus into a grid of (small) squares $\alpha$ of side length $b$. To be concrete, we center the grid such that the small square containing $0 \in[1 / 2,1 / 2)^{2}$ has 0 as its center. We denote the set of these squares by $\mathbf{S}_{0}$.

For $\ell \ll b \ll 1$, the quasi-free Yukawa interaction is obtained from the Yukawa interaction by, roughly speaking, removing the interaction between particles in a small square with particles outside that square and replacing the interaction between particles in the same square by a periodic one. More precisely, we denote by $\mathbf{n}=\left(n_{\alpha}\right)$ a particle profile, by which we mean an assignment of a number of particles $n_{\alpha} \in \mathbb{N}$ to each square $\alpha$, with the constraint $\sum_{\alpha} n_{\alpha}=N$ where sums over $\alpha$ are always over $\alpha \in \mathbf{S}_{0}$. We associate a torus $\mathbb{T}_{\alpha}$ of side length $b$ to each square $\alpha$. The tori $\mathbb{T}_{\alpha}$ are of course all identical and equal to $\mathbb{T}^{(b)}$, but we keep the index $\alpha$ to emphasize the connection with the square it is associated to, and label elements in $\mathbb{T}_{\alpha}$ by $(\alpha, z)$ with $z \in \mathbb{T}^{(b)}$. For $v=(\alpha, z) \in \mathbb{T}_{\alpha}$ we write $U_{b}^{\ell}(v)=U_{b}^{\ell}(z)$ where $U_{b}^{\ell}(v)$ is the periodic Yukawa interaction on $\mathbb{T}^{(b)}$ defined in (3.18).

For $n_{\alpha} \in \mathbb{N}$, we define

$$
\hat{H}_{\alpha}(\mathbf{v})=\sum_{i \neq j} U_{b}^{\ell}\left(v_{i}-v_{j}\right) \quad\left(\mathbf{v} \in \mathbb{T}_{\alpha}^{n_{\alpha}}\right)
$$


Given a particle profile $\mathbf{n}$, the quasi-free free energy with particle profile $\mathbf{n}$ is defined by

$$
F(\mathbf{n})=\frac{1}{\beta} \log \left(\begin{array}{l}
N \\
\mathbf{n}
\end{array}\right)+\frac{1}{\beta} \sum_{\alpha} \log \int_{\mathbb{T}_{\alpha}^{n_{\alpha}}} \mathrm{e}^{-\beta \hat{H}_{\alpha}(\mathbf{u})} m(\mathrm{~d} \mathbf{u})
$$

where the term $\left(\begin{array}{l}N \\ \mathbf{n}\end{array}\right)=\frac{N !}{\prod_{\alpha} n_{\alpha} !}$ arises as the number of ways to distribute $N$ particles into groups of sizes $\left(n_{\alpha}\right)$ with $\sum_{\alpha} n_{\alpha}=N$.

The name quasi-free is motivated by the fact that particles in different squares do not interact, i.e., their contribution is additive. The following two propositions show that its free energy is a good approximation to that of the original Yukawa gas. To state the second proposition, denote by $\overline{\mathbf{n}}=\left(\bar{n}_{\alpha}\right)$ with $\bar{n}_{\alpha}=\bar{n}=N b^{2}$ the expected number of particles in the square $\alpha$.

Proposition 3.8 (Upper Bound). Assume that $\ell \ll b \ll 1$. Then

$$
\frac{1}{\beta} \log \int \mathrm{e}^{-\beta H^{\ell}(\mathbf{z})} m(\mathrm{~d} \mathbf{z}) \leqslant \frac{1}{\beta} \log \sum_{\mathbf{n}} \mathrm{e}^{\beta F(\mathbf{n})}+N^{\varepsilon} \mathrm{O}\left(N^{2} \ell^{3} b^{-1}\right) .
$$

Proposition 3.9 (Lower Bound). Assume that $\ell \ll b \ll 1$. Then

$$
\frac{1}{\beta} \log \int \mathrm{e}^{-\beta H^{\ell}(\mathbf{z})} m(\mathrm{~d} \mathbf{z}) \geq F(\overline{\mathbf{n}})+N^{\varepsilon} \mathrm{O}\left(N^{2} \ell^{3} b^{-1}\right) .
$$

We will prove the two propositions in the next two sections.

3.5. Upper bound: proof of Proposition 3.8, By translation invariance, instead of working with the grid of squares $\mathbf{S}_{0}$ centered at 0 , we can equivalently consider the shifted grid consisting of square $u+\alpha$ with $\alpha \in \mathbf{S}_{0}$ and center $u \in[-b / 2, b / 2)^{2}$. The center of $u+\alpha$ is $c(u+\alpha)=u+c(\alpha)$. Given this choice of origin $u$ and a square $\alpha \in \mathbf{S}_{0}$, we define

$$
\Phi_{\alpha} \equiv \Phi_{\alpha}^{u}: u+\alpha \rightarrow \mathbb{T}^{(b)} \text { the natural embedding from the square } u+\alpha \text { into the torus } \mathbb{T}^{(b)},
$$

mapping the boundary of $u+\alpha$ to a vertical and a horizontal line in $\mathbb{T}^{(b)}$. More precisely, using the coordinates $c(u+\alpha)+[-b / 2, b / 2)^{2}$ on the square $u+\alpha \subset \mathbb{T}$ and the coordinates $[-b / 2, b / 2)^{2}$ on the torus $\mathbb{T}^{(b)}$, we set

$$
\Phi_{\alpha}^{u}(z)=z-c(u+\alpha) .
$$

For $z, w$ in the original unit torus $\mathbb{T}$, we define the quasi-free pair interaction through the embeddings $\Phi_{\alpha}$ by

$$
\tilde{Y}_{u}^{\ell}(z, w)=\sum_{\alpha \in \mathbf{S}_{0}} U_{b}^{\ell}\left(\Phi_{\alpha}^{u}(z)-\Phi_{\alpha}^{u}(w)\right) \mathbb{1}_{z \in u+\alpha} \mathbb{1}_{w \in u+\alpha} \quad(z, w \in \mathbb{T}) .
$$

The interaction $\tilde{Y}_{u}^{\ell}$ is in fact very simple: we divide the unit torus into a grid of cubes of side length $b$ with the grid centered at $u$. Then for two particles in the same small square $\alpha$, we view them as two points on the torus $\mathbb{T}^{(b)}$ interacting via the torus Yukawa potential $U_{b}^{\ell}$. For two particles in different small squares, the interaction vanishes.

The corresponding Hamiltonian $\tilde{H}_{u}^{\ell}$ with pair interaction $\tilde{Y}_{u}^{\ell}$ is

$$
\tilde{H}_{u}^{\ell}(\mathbf{z})=\sum_{i \neq j} \tilde{Y}_{u}^{\ell}\left(z_{i}, z_{j}\right) \quad\left(\mathbf{z} \in \mathbb{T}^{N}\right)
$$


The choice of origin $u \in[-b / 2, b / 2)^{2}$ was arbitrary and we will eventually average of this choice. We set $\mathbb{E}^{u} f(u)=\frac{1}{b^{2}} \int_{[-b / 2, b / 2)^{2}} \mathrm{~d} u f(u)$ and define the function $\bar{Y}$ by

$$
\bar{Y}(z, w)=\mathbb{E}^{u} \tilde{Y}_{u}^{\ell}(z, w) \quad(z, w \in \mathbb{T}) .
$$

By Jensen's inequality and then averaging over $u$,

$$
\frac{1}{\beta} \log \int \mathrm{e}^{-\beta H^{\ell}(\mathbf{z})} m(\mathrm{~d} \mathbf{z}) \leq \frac{1}{\beta} \mathbb{E}^{u} \log \int \mathrm{e}^{-\beta \tilde{H}_{u}^{\ell}(\mathbf{z})} m(\mathrm{~d} \mathbf{z})+\mathbb{E}^{u} \mathbb{E}^{H^{\ell}}\left(\tilde{H}_{u}^{\ell}-H^{\ell}\right) .
$$

The second term on the right-hand side is $\mathbb{E}^{H^{\ell}} \sum_{i \neq j}\left[\bar{Y}\left(z_{i}, z_{j}\right)-U^{\ell}\left(z_{i}-z_{j}\right)\right]$. This expression is estimated in Lemma 3.11 below. Its proof follows by counting particles using the local density estimate.

In preparation for the proof, we first state some estimates on $\bar{Y}$. These estimates are stated in terms of the function $g: \mathbb{C} \rightarrow \mathbb{R}$ defined with $z=x+\mathrm{i} y$ by

$$
g(z)=\frac{(b-|x|)_{+}(b-|y|)_{+}}{b^{2}} Y^{\ell}\left(\sqrt{|x|_{b}^{2}+|y|_{b}^{2}}\right), \quad|x|_{b}=|x| \wedge(b-|x|)_{+},
$$

where we write $Y(r)$ for $Y(z)$ with $|z|=r$. By definition, $g$ is supported in $[-b, b]^{2}$. Assuming an identification of $\mathbb{T}$ with $[-1 / 2,1 / 2)^{2}, g$ can be extended to a function on $\mathbb{T}$. Thus $g(z-w)$ is well-defined as a function on $\mathbb{T} \times \mathbb{T}$.

Lemma 3.10. Assume that $\ell \ll b$. Then

$$
\bar{Y}(z, w)=g(z-w)+\mathrm{O}\left(\mathrm{e}^{-c b / \ell}\right) \quad(z, w \in \mathbb{T})
$$

and

$$
U^{\ell}(z-w)=g(z-w)+\mathrm{O}(\ell / b) \quad(z, w \in \mathbb{T}) .
$$

Proof. We first verify (3.33), i.e., we evaluate $\bar{Y}(z, w)$. If $|z-w|_{\infty}>b$ then the points $z$ and $w$ are necessarily in two different squares and $\tilde{Y}_{u}^{\ell}(z, w)=0$ by (3.28). Thus we can assume that $|z-w|_{\infty} \leqslant b$. We write $z-w=x+\mathrm{i} y$ with $x, y \in[-b, b]$

For fixed such $z, w$, the probability under the average over $u$ that $z$ and $w$ lie in the same square is given by $(b-|x|) / b \times(b-|y|) / b$. For $z, w$ in the same square, we have $U_{b}^{\ell}(\Phi(z)-\Phi(w))=$ $Y^{\ell}\left(\sqrt{x^{2}+y^{2}}\right)+\mathrm{O}\left(\mathrm{e}^{-c b / \ell}\right)$ where the error is from the sum over the periods in the definition of $U_{b}^{\ell}$. We have thus proved (3.33), i.e.,

$$
\bar{Y}(z, w)=\mathbb{E}^{u} \tilde{Y}_{u}^{\ell}(z, w)=\frac{(b-|x|)(b-|y|)}{b^{2}} Y^{\ell}\left(\sqrt{x^{2}+y^{2}}\right)+\mathrm{O}\left(\mathrm{e}^{-c / \gamma}\right) .
$$

To verify (3.34), first assume that $|x| \vee|y| \leqslant b$. Then, by the definition (3.32) and using the exponential decay $Y^{\ell}(z-w)=O\left(\mathrm{e}^{-c|z-w| / \ell}\right)$,

$$
g(z-w)-Y^{\ell}(z-w)=O(|z-w| / b) Y^{\ell}(z-w)=O(\ell / b) .
$$

On the other hand, if $|x| \vee|y| \geqslant b$ then $g(x, y)=0$ and the claim follows from

$$
\left|g(z-w)-Y^{\ell}(z-w)\right|=Y^{\ell}(z-w)=O\left(\mathrm{e}^{-c|z-w| / \ell}\right) \leqslant O\left(\mathrm{e}^{-c b / \ell}\right) \leqslant O(\ell / b),
$$

using the assumption $\ell \ll b$. This completes the proof. 
Lemma 3.11. Assume that $\ell \ll b \ll 1$. Then

$$
\mathbb{E}^{H^{\ell}} \sum_{i, j}\left[\bar{Y}\left(z_{i}-z_{j}\right)-U^{\ell}\left(z_{i}-z_{j}\right)\right]=N^{\varepsilon} \mathrm{O}\left(N^{2} \ell^{3} b^{-1}\right) .
$$

Proof. We use the local density for the Yukawa gas, Theorem 2.2, implying that any square in $\mathbb{T}$ of diameter $r \gg N^{-1 / 2}$ contains $\mathrm{O}\left(N r^{2}\right)$ particles with high probability. In addition, $Y^{\ell}\left(z_{i}-z_{j}\right) \leqslant \mathrm{e}^{-c N^{\varepsilon}}$ if $\left|z_{i}-z_{j}\right| \geq \ell N^{\varepsilon}$. Thus $\bar{Y}\left(z_{i}-z_{j}\right)+U^{\ell}\left(z_{i}-z_{j}\right) \leqslant \mathrm{O}\left(N^{-\infty}\right)$ in this case and the sum over the contributions of these terms in (3.37) is again of order $\mathrm{O}\left(N^{-\infty}\right)$. Therefore, we can assume that $\left|z_{i}-z_{j}\right| \leq \ell N^{\varepsilon}$ for all $i, j$ from now on. By using (3.33), we can replace $\bar{Y}$ by $g$. As a consequence,

$$
\mathbb{E}^{H^{\ell}} \sum_{i, j}\left[g\left(z_{i}-z_{j}\right)-U^{\ell}\left(z_{i}-z_{j}\right)\right]=\mathrm{O}\left(N^{\varepsilon} N\left(N \ell^{2}\right)(\ell / b)\right)
$$

since each of the at most $N$ particles $z_{i}$ interacts with $\mathrm{O}\left(N^{\varepsilon} N \ell^{2}\right)$ particles $z_{j}$, and the difference $U^{\ell}(z-w)-g(z-w)$ is of order $\ell / b$ by Lemma 3.10. This proves (3.37).

Proof of Proposition 3.8. By (3.31) and (3.37),

$$
\frac{1}{\beta} \log \int \mathrm{e}^{-\beta H^{\ell}(\mathbf{z})} m(\mathrm{~d} \mathbf{z}) \leq \frac{1}{\beta} \mathbb{E}^{u} \log \int \mathrm{e}^{-\beta \tilde{H}_{u}^{\ell}(\mathbf{z})} m(\mathrm{~d} \mathbf{z})+O\left(N^{\varepsilon} N^{2} \ell^{3} b^{-1}\right) .
$$

By the definitions (3.28)-(3.29), after partitioning in the number of particles in each square, the integral in the first term on the right-hand side factorizes over the squares and therefore is

$$
\int \mathrm{e}^{-\beta \tilde{H}_{u}^{\ell}(\mathbf{z})} m(\mathrm{~d} \mathbf{z})=\sum_{\mathbf{n}}\left(\begin{array}{l}
N \\
\mathbf{n}
\end{array}\right) \prod_{\alpha} \int_{\mathbb{T}_{\alpha}^{n_{\alpha}}} \mathrm{e}^{-\beta \hat{H}_{\alpha}(\mathbf{u})} m(\mathrm{~d} \mathbf{u})=\sum_{\mathbf{n}} \mathrm{e}^{\beta F(\mathbf{n})},
$$

where we used the definition (3.23). This completes the proof.

3.6. Lower bound: proof of Proposition 3.9. To obtain a lower bound on the partition function, we use the coordinates $[-1 / 2,1 / 2)^{2}$ for $\mathbb{T}$ and the grid $\mathbf{S}$ centered at 0 . We can then restrict the particle numbers in all squares $\alpha$ to their mean $\bar{n}=\bar{n}_{\alpha}=N b^{2}$. (Although $\bar{n}_{\alpha}$ is independent of $\alpha$, we often keep the index for analogy with Section 4.) Thus we define the indicator function

$$
\hat{\chi}(\mathbf{z})=\prod_{\alpha} \mathbf{1}\left(n_{\alpha}(\mathbf{z})=\bar{n}_{\alpha}\right),
$$

where, for a particle configuration $\mathbf{z} \in \mathbb{T}^{N}$, we define $\mathbf{n}(\mathbf{z})=\left(n_{\alpha}(\mathbf{z})\right)$ to be the particle profile associated to the configuration $\mathbf{z}$, i.e., $n_{\alpha}(\mathbf{z})$ is the number of particles $z_{j} \in \alpha$. Trivially,

$$
\frac{1}{\beta} \log \int \mathrm{e}^{-\beta H^{\ell}(\mathbf{z})} m(\mathrm{~d} \mathbf{z}) \geq \frac{1}{\beta} \log \int \mathrm{e}^{-\beta H^{\ell}(\mathbf{z})} \hat{\chi}(\mathbf{z}) m(\mathrm{~d} \mathbf{z}) .
$$

Ordering the squares in $\mathbf{S}$ arbitrarily as $\alpha_{1}, \alpha_{2}, \ldots$, we write $\tilde{\chi}(\mathbf{z})$ for $\hat{\chi}(\mathbf{z})$ multiplied by the indicator function of the event in which the particles $z_{1}, \ldots, z_{\bar{n}}$ are in $\alpha_{1}$, the particles $z_{\bar{n}+1}, \ldots, z_{2 \bar{n}}$ are in $\alpha_{2}$, and so forth. Then

$$
\frac{1}{\beta} \log \int \mathrm{e}^{-\beta H^{\ell}(\mathbf{z})} \hat{\chi}(\mathbf{z}) m(\mathrm{~d} \mathbf{z})=\frac{1}{\beta} \log \left(\begin{array}{c}
N \\
\mathbf{n}
\end{array}\right)+\frac{1}{\beta} \log \int \mathrm{e}^{-\beta H^{\ell}(\mathbf{z})} \tilde{\chi}(\mathbf{z}) m(\mathrm{~d} \mathbf{z}),
$$

where $\left(\begin{array}{l}N \\ \mathbf{n}\end{array}\right)$ is the combinatorial factor for dividing $N$ particles into small cubes of size $n_{1}, n_{2}, \ldots$ 
To estimate the integral on the right-hand side, we choose maps

$$
\Psi_{\alpha}: \mathbb{T}^{(b)} \rightarrow \alpha
$$

that embed the torus $\mathbb{T}^{(b)}$ injectively into the square $\alpha$. Note that the maps $\Psi_{\alpha}$ go in the opposite direction of the maps $\Phi_{\alpha}$ in (3.26) used in the upper bound. Such an embedding is necessarily discontinuous along a horizontal and a vertical line in the image. We will choose the maps $\Psi_{\alpha}$ randomly by averaging over the positions of the discontinuity lines. The center where the two discontinuity lines meet can be parametrized by a point $u \in[-b / 2, b / 2)^{2}$. Using the coordinates $[1 / 2,1 / 2)^{2}$ on the unit torus and recalling that $\mathbf{S}$ is the grid of size $b$ with center 0 , we can parametrize the square $\alpha \in \mathbf{S}$ by $c(\alpha)+[-b / 2, b / 2)^{2}$ where $c(\alpha) \in(b \mathbb{Z})^{2}$ is the center of $\alpha$. Using $[-b / 2, b / 2)^{2}$ as coordinates for $\mathbb{T}^{(b)}$, we define $\Psi_{\alpha}^{u}$ by

$$
\Psi_{\alpha}^{u}(w)=c(\alpha)+[u+w], \quad\left(w \in[-b / 2, b / 2)^{2}\right),
$$

where $[z]$ the representative of $z \in \mathbb{C}$ in $[-b / 2, b / 2)^{2}$ modulo $(b \mathbb{Z})^{2}$. Clearly, the maps $\Psi_{\alpha}^{u}$ have Jacobian $\left|\mathrm{d} \Psi_{\alpha}^{u}\right|=1$. We write $\mathbb{E}^{\Psi}$ for the average over $u$.

Given the particle profile $\mathbf{n}=\overline{\mathbf{n}}=\left(\bar{n}_{\alpha}\right)$ and $\hat{H}_{\alpha}$ defined in 3.22 , set

$$
\hat{H}(\mathbf{v})=\sum_{\alpha} \hat{H}_{\alpha}\left(\mathbf{v}^{\alpha}\right)
$$

Let $\omega_{\alpha}$ be the probability measure of the Yukawa gas on $\left(\mathbb{T}^{(b)}\right)^{n_{\alpha}}$ and $\boldsymbol{\omega}$ their product:

$$
\omega_{\alpha}\left(\mathrm{d} \mathbf{v}^{\alpha}\right)=\frac{1}{Z_{\alpha}} \mathrm{e}^{-\beta \hat{H}_{\alpha}\left(\mathbf{v}^{\alpha}\right)} m\left(\mathrm{~d} \mathbf{v}^{\alpha}\right), \quad \boldsymbol{\omega}=\prod_{\alpha} \omega_{\alpha} .
$$

Moreover, given the maps $\Psi_{\alpha}$, define $\Psi$ by

$$
\Psi: \prod_{\alpha}\left(\mathbb{T}^{(b)}\right)^{n_{\alpha}} \rightarrow \mathbb{T}^{N}, \quad \Psi(\{\mathbf{v}\})=\left(\left\{\Psi_{\alpha}\left(\mathbf{v}^{\alpha}\right)\right\}\right) \in \mathbb{T}^{N},
$$

where configurations in the image of $\Psi$ have $n_{\alpha}=\bar{n}_{\alpha}$ particles in the square $\alpha$ (in some fixed order that is irrelevant), and $\Psi^{*} \boldsymbol{\omega}=\prod_{\alpha} \Psi_{\alpha}^{*} \boldsymbol{\omega}_{\alpha}$ is a measure on such configurations of $N$ particles in $\mathbb{T}$.

For such a configuration $\mathbf{z}$, we write $\mathbf{z}^{\alpha}$ for the vector of particles in the square $\alpha$. Then defining $\hat{H}_{\Psi}(\mathbf{z})=\sum_{\alpha} \hat{H}_{\alpha} \circ \Psi_{\alpha}^{-1}\left(\mathbf{z}^{\alpha}\right)$, we have by Jensen's inequality,

$$
\frac{1}{\beta} \log \int \mathrm{e}^{-\beta H^{\ell}(\mathbf{z})} \tilde{\chi}(\mathbf{z}) m(\mathrm{~d} \mathbf{z}) \geq \frac{1}{\beta} \log \int \mathrm{e}^{-\beta \hat{H}_{\Psi}(\mathbf{z})} \tilde{\chi}(\mathbf{z}) m(\mathrm{~d} \mathbf{z})+\mathbb{E}^{\Psi^{*} \boldsymbol{\omega}}\left(\hat{H}_{\Psi}-H^{\ell}\right) .
$$

Reversing the change of variables and averaging over the distribution of maps $\Psi$ with $|\mathrm{d} \Psi|=1$, whose expectation is denoted by $\mathbb{E}^{\Psi}$, we have

$$
\frac{1}{\beta} \log \int \mathrm{e}^{-\beta H^{\ell}} \tilde{\chi} \geq \frac{1}{\beta} \log \int \mathrm{e}^{-\beta \hat{H}_{\alpha}\left(\mathbf{v}^{\alpha}\right)} \prod_{\alpha} d \mathbf{v}^{\alpha}+\mathbb{E}^{\Psi} \mathbb{E}^{\omega}\left(\hat{H}(\mathbf{v})-H^{\ell}(\Psi(\mathbf{v}))\right) .
$$

It remains to estimate the second term on right-hand side of (3.48). Let $\mu_{\alpha}$ denote either the normalized uniform measure on the square $\alpha$ or the associated torus $\mathbb{T}_{\alpha}$ (the distinction will be clear from the context). We write $\tilde{\mu}_{\alpha}=\hat{\mu}_{\alpha}-\mu_{\alpha}$ where

$$
\hat{\mu}_{\alpha}(\mathrm{d} v)=\frac{1}{n_{\alpha}} \sum_{j: v_{j} \in \mathbb{T}_{\alpha}} \delta_{v_{j}}(\mathrm{~d} v) .
$$


Note that $\int \mathrm{d} \hat{\mu}_{\alpha}=1$. Define

$$
E=\sum_{\alpha} \bar{n}_{\alpha}^{2} \mathbb{E}^{\Psi} \mathbb{E}^{\omega} \iint_{\mathbb{T}_{\alpha} \times \mathbb{T}_{\alpha}}\left(U_{b}^{\ell}(v-w)-Y^{\ell}\left(\Psi_{\alpha}(v)-\Psi_{\alpha}(w)\right)\right) \tilde{\mu}_{\alpha}(\mathrm{d} v) \tilde{\mu}_{\alpha}(\mathrm{d} w) .
$$

The following two lemmas, which we will prove in the remainder of this section, estimate the second term on right-hand side of (3.48).

Lemma 3.12. Assume $\ell \ll b \ll 1$. Then

$$
\mathbb{E}^{\Psi} \mathbb{E}^{\boldsymbol{\omega}}\left(\hat{H}(\mathbf{v})-H^{\ell}(\Psi(\mathbf{v}))\right)=E+\mathrm{O}\left(N^{-\infty}\right) .
$$

Lemma 3.13. Assume $\ell \ll b \ll 1$. Then

$$
E=N^{\varepsilon} \mathrm{O}\left(N^{2} \ell^{3} b^{-1}\right) .
$$

Given Lemmas 3.123 .13 , the proof of Proposition 3.9 is completed as follows.

Proof of Proposition 3.9. By combining (3.40)-(3.52), we have

$$
\frac{1}{\beta} \log \int \mathrm{e}^{-\beta H^{\ell}(\mathbf{z})} m(\mathrm{~d} \mathbf{z}) \geqslant \frac{1}{\beta} \log \left(\begin{array}{c}
N \\
\mathbf{n}
\end{array}\right)+\sum_{\alpha} \frac{1}{\beta} \log \int \mathrm{e}^{-\beta \hat{H}_{\alpha}\left(\mathbf{v}^{\alpha}\right)} d \mathbf{v}^{\alpha}+N^{\varepsilon} \mathrm{O}\left(N^{2} \ell^{3} b^{-1}\right) .
$$

The first two terms on the right-hand side of $(3.53)$ together equal $F(\overline{\mathbf{n}})$, completing the proof.

To complete the proof of Proposition 3.9, it still remains to prove Lemmas 3.123 .13 . Proof of Lemma 3.12. We must prove that

$$
\sum_{\alpha, \beta} \mathbb{E}^{\Psi} \mathbb{E}^{\boldsymbol{\omega}}\left[\sum_{i \neq j} \mathbf{1}_{v_{i} \in \mathbb{T}_{\alpha}} \mathbf{1}_{v_{j} \in \mathbb{T}_{\beta}}\left(U_{b}^{\ell}\left(v_{i}-v_{j}\right) \mathbb{1}_{\alpha=\beta}-Y^{\ell}\left(\Psi_{\alpha}\left(v_{i}\right)-\Psi_{\beta}\left(v_{j}\right)\right)\right)\right]=E+\mathrm{O}\left(N^{-\infty}\right) .
$$

We first note that the contribution of the nonadjacent squares on the left-hand side is bounded by $\mathrm{O}\left(\mathrm{e}^{-c \ell / b}\right)=\mathrm{O}\left(N^{-\infty}\right)$, by $(3.18)$ and $(2.2)$. For any $\alpha, \beta$ (including $\alpha=\beta$ ), define

$$
\bar{Y}_{\alpha \beta}=\iint_{\mathbb{T}_{\alpha} \times \mathbb{T}_{\beta}} Y^{\ell}\left(\Psi_{\alpha}(v)-\Psi_{\beta}(w)\right) \mu_{\alpha}(\mathrm{d} v) \mu_{\beta}(\mathrm{d} w)=\iint_{\alpha \times \beta} Y^{\ell}(v-w) \mu_{\alpha}(\mathrm{d} v) \mu_{\beta}(\mathrm{d} w),
$$

where the equality follows from $\mathbb{E}^{\Psi} \mathbb{E}^{\omega} \hat{\mu}_{\alpha}(\mathrm{d} v)=\mu_{\alpha}(\mathrm{d} v)$ and $\left|\mathrm{d} \Psi_{\alpha}\right|=\left|\mathrm{d} \Psi_{\beta}\right|=1$. Denoting by $\alpha \sim \beta$ that the squares $\alpha$ and $\beta$ are adjacent, therefore

$$
\begin{aligned}
& \sum_{\alpha, \beta} \mathbb{E}^{\Psi} \mathbb{E}^{\omega} {\left[\sum_{i \neq j} \mathbf{1}_{v_{i} \in \mathbb{T}_{\alpha}} \mathbf{1}_{v_{j} \in \mathbb{T}_{\beta}}\left(U_{b}^{\ell}\left(v_{i}-v_{j}\right) \mathbb{1}_{\alpha=\beta}-Y^{\ell}\left(\Psi_{\alpha}\left(v_{i}\right)-\Psi_{\beta}\left(v_{j}\right)\right)\right)\right] } \\
&=\sum_{\alpha} \bar{n}_{\alpha}^{2} \mathbb{E}^{\Psi} \mathbb{E}^{\omega}\left[\iint_{\mathbb{T}_{\alpha} \times \mathbb{T}_{\alpha}}\left(U_{b}^{\ell}(v-w)-Y^{\ell}\left(\Psi_{\alpha}(v)-\Psi_{\alpha}(w)\right)\right) \hat{\mu}_{\alpha}(\mathrm{d} v) \hat{\mu}_{\alpha}(\mathrm{d} w)\right] \\
& \quad-\sum_{\alpha \sim \beta} \bar{n}_{\alpha} \bar{n}_{\beta} \bar{Y}_{\alpha \beta}+\mathrm{O}\left(N^{-\infty}\right) .
\end{aligned}
$$

Using again $\left|\mathrm{d} \Psi_{\alpha}\right|=1$ and $\mathbb{E}^{\Psi} \mathbb{E}^{\omega} \tilde{\mu}_{\alpha}=0$, we can rewrite the difference of the first term in the last line and $E$ as

$$
\sum_{\alpha} \bar{n}_{\alpha}^{2}\left[\iint_{\mathbb{T}_{\alpha}^{2}} U_{b}^{\ell}(v-w) \mu_{\alpha}(\mathrm{d} v) \mu_{\alpha}(\mathrm{d} w)-\iint_{\alpha^{2}} Y^{\ell}(v-w) \mu_{\alpha}(\mathrm{d} v) \mu_{\alpha}(\mathrm{d} w)\right] .
$$


We then apply the cancellation (3.58) below to this term and the last term on the right-hand side of 3.56, i.e., $-\sum_{\alpha \sim \beta} \bar{n}_{\alpha} \bar{n}_{\beta} \bar{Y}_{\alpha \beta}$. Finally, we sum over the squares $\alpha$ of which there are $\mathrm{O}\left(b^{-2}\right)$ many. Using $\mathrm{O}\left(b^{-2}\right) \mathrm{O}\left(N^{-\infty}\right)=\mathrm{O}\left(N^{-\infty}\right)$ and the definition of $E$, the claim follows.

Lemma 3.14. For any square $\alpha$ of side length $b \gg \ell$ fixed,

$$
\begin{aligned}
\bar{n}_{\alpha}^{2}\left[\iint_{\mathbb{T}_{\alpha}^{2}} U_{b}^{\ell}(v-w) \mu_{\alpha}(\mathrm{d} v) \mu_{\alpha}(\mathrm{d} w)-\iint_{\alpha^{2}} Y^{\ell}(v-w) \mu_{\alpha}(\mathrm{d} v) \mu_{\alpha}(\mathrm{d} w)\right] & \\
& -\sum_{\beta: \beta \sim \alpha} \bar{n}_{\alpha} \bar{n}_{\beta} \bar{Y}_{\alpha \beta}=\mathrm{O}\left(N^{-\infty}\right) .
\end{aligned}
$$

Proof. Using that contributions of pairs with distance $\gg \ell / b$ are negligible in $U_{b}^{\ell}$ and unfolding the periodization in the definition of $U_{b}^{\ell}$, we have

$$
\iint_{\mathbb{T}_{\alpha}^{2}} U_{b}^{\ell}(u-v) m(\mathrm{~d} v) m(\mathrm{~d} w)=\int_{\alpha} \int_{\left(\cup_{\beta \sim \alpha} \beta\right) \cup \alpha} Y^{\ell}(z-w) m(\mathrm{~d} z) m(\mathrm{~d} w)+\mathrm{O}\left(N^{-\infty}\right) .
$$

Thus the first two terms on the left-hand side of (3.58) are given by

$$
\sum_{\beta \sim \alpha} \iint_{\alpha \times \beta} Y^{\ell}(z-w) \mu_{\alpha}(\mathrm{d} z) \mu_{\beta}(\mathrm{d} w)+\mathrm{O}\left(N^{-\infty}\right)
$$

i.e., the left-hand side of (3.58) equals

$$
\bar{n}_{\alpha} \sum_{\beta \sim \alpha}\left(\bar{n}_{\alpha}-\bar{n}_{\beta}\right) \iint_{\alpha \times \beta} Y^{\ell}(z-w) \mu_{\alpha}(\mathrm{d} z) \mu_{\beta}(\mathrm{d} w)+\mathrm{O}\left(\mathrm{e}^{-N^{\varepsilon}}\right)=\mathrm{O}\left(N^{-\infty}\right)
$$

as needed.

Proof of Lemma 3.13. By the definition 3.50, $\bar{n}_{\alpha}=N b^{2}$, and since there are $b^{-2}$ squares $\alpha$, we must bound

$$
E=b^{-2}\left(N b^{2}\right)^{2} \mathbb{E}^{\Psi} \mathbb{E}^{\omega} \iint_{\mathbb{T}^{(b)} \times \mathbb{T}^{(b)}}\left(U_{b}^{\ell}(v-w)-Y^{\ell}\left(\Psi_{\alpha}(v)-\Psi_{\alpha}(w)\right)\right) \tilde{\mu}_{\alpha}(\mathrm{d} v) \tilde{\mu}_{\alpha}(\mathrm{d} w),
$$

where $\alpha$ is any of the squares in $\mathbf{S}$. Recall that the expectation $\mathbb{E}^{\Psi} \mathbb{E}^{\omega}$ averages over the parameter $u \in[-b / 2, b / 2)^{2}$ in the definition of the embedding $\Psi_{\alpha}^{u}$ (see Section 3.4 ) and over the Yukawa gas in $\mathbb{T}_{\alpha}$. We estimate this expectation in two steps.

Step 1. For $v, w \in \mathbb{T}^{(b)}$, we write $v-w=(x, y)$ in the sense that $(x, y) \in \mathbb{R}^{2}$ is a representative for $v-w \in \mathbb{T}^{(b)}$ chosen such that $|x| \leqslant b / 2$ and $|y| \leqslant b / 2$. We claim that

$$
\mathbb{E}^{\Psi}\left(U_{b}^{\ell}(v-w)-Y^{\ell}\left(\Psi_{\alpha}(v)-\Psi_{\alpha}(w)\right)\right)=\frac{b|x|+b|y|-|x y|}{b^{2}} U_{b}^{\ell}(v-w)+\mathrm{O}\left(\mathrm{e}^{-c b / \ell}\right) .
$$

The proof of 3.60 uses exactly the same reasoning as that of Lemma 3.10. Namely, by the definitions of $\Psi_{\alpha}$ and $U_{b}^{\ell}$, the difference $U_{b}^{\ell}(v-w)-Y^{\ell}\left(\Psi_{\alpha}(v)-\Psi_{\alpha}(w)\right)$ is $\mathrm{O}\left(\mathrm{e}^{-c b / \ell}\right)$ unless $v$ and $w$ have periodic distance of order $\ell$ and $\Psi_{\alpha}(v)$ and $\Psi_{\alpha}(w)$ have Euclidean distance order $b$ (i.e., $\Psi_{\alpha}(v)$ and $\Psi_{\alpha}(w)$ are on opposite sides of the square $\alpha$ ). Assuming that the difference is not $\mathrm{O}\left(e^{-c b / \ell}\right)$, the $Y$ term itself is $\mathrm{O}\left(\mathrm{e}^{-c b / \ell}\right)$, and only the $U$ term contributes. The prefactor $(b|x|+b|y|-|x y|) / b^{2}$ on the right-hand side of $(3.60)$ is the probability that $\Psi_{\alpha}(v)$ and $\Psi_{\alpha}(w)$ fall on opposite sides of the torus under the randomness of $\mathbb{E}^{\Psi}$, i.e., when the center of the square $\alpha$ is chosen uniformly. 
Step 2. We claim that

$$
\mathbb{E}^{\boldsymbol{\omega}} \iint \frac{b|x|+b|y|-|x y|}{b^{2}} U_{b}^{\ell}(v-w) \tilde{\mu}(\mathrm{d} v) \tilde{\mu}(\mathrm{d} w)=n^{\varepsilon} \mathrm{O}(\ell / b)^{3} .
$$

This estimate does not use any cancellations due to the difference in the definition of $\tilde{\mu}_{\alpha}$ as $\hat{\mu}_{\alpha}-\tilde{\mu}_{\alpha}$. We may therefore replace $\tilde{\mu}$ by $\hat{\mu}$; the terms involving $\mu_{V}$ obtained when expanding $\tilde{\mu}$ are analogous. Moreover, since the left-hand side of (3.61) does not depend on the position of the center of the square $\alpha$, the expectation $\mathbb{E}^{\Psi} \mathbb{E}^{\omega}$ can be replaced by the expectation of the Yukawa gas on the torus $\mathbb{T}^{(b)}$. Furthermore, by rescaling it suffices to assume that $b=1$, i.e., that the torus $\mathbb{T}^{(b)}$ is the unit torus $\mathbb{T}$. With $\gamma=\ell / b$ and denoting by $\mathbb{E}^{\gamma}$ the expectation of the Yukawa gas on the unit torus with $n$ particles and range $\gamma$, it is then sufficient to to show that

$$
n^{2} \mathbb{E}^{\gamma} \iint|v-w| U^{\gamma}(v-w) \hat{\mu}(\mathrm{d} v) \hat{\mu}(\mathrm{d} w)=n^{\varepsilon} \mathrm{O}\left(n^{2} \gamma^{3}\right)
$$

Note that $\mathrm{O}\left(n^{2} \gamma^{3}\right)$ is the order of the left-hand side when we replace $\hat{\mu}$ by the uniform measure. So the proof of the last bound can be understood by the simple heuristic that the density of the measure $\hat{\mu}$ is bounded by uniform measure at the scale $\gamma$ provided by the regularization of the interaction $U^{\gamma}$. We now give the formal proof by using the local density bound for $\hat{\mu}$ stated in Theorem 2.2. More precisely, dividing the unit torus into squares of length $\tilde{b}=n^{\varepsilon} \gamma$, by the local density estimate, each square contains $\mathrm{O}\left(n \tilde{b}^{2}\right)$ particles, with high probability. Thus, denoting the squares by $\alpha$ and $\beta$, the left-hand side of (3.61) is bounded by

$$
n^{2} \mathbb{E}^{\gamma} \sum_{\alpha, \beta} \iint_{\alpha \times \beta}|v-w| U^{\gamma}(v-w) \hat{\mu}(\mathrm{d} v) \hat{\mu}(\mathrm{d} w) .
$$

Using the exponential decay of $U^{\gamma}(v-w)$, up to an error of order $\mathrm{O}\left(\mathrm{e}^{-c n^{\varepsilon}}\right)$, only the neighboring or equal pairs of squares $\alpha, \beta$ contribute to this sum. For each such pair, the contribution is $\mathrm{O}\left(n^{2} \tilde{b}^{5}\right)$ with two factors of $\tilde{b}^{2}$ arising from the integrals over $z$ and $w$ and one from the factor $|z-w|$. Summing over the $\mathrm{O}\left(\tilde{b}^{-2}\right)$ terms and using that $\tilde{b}=n^{\varepsilon} \gamma$, the left-hand side of (3.62) is bounded by $\mathrm{O}\left(n^{2} \tilde{b}^{3}\right)=\mathrm{O}\left(n^{2+3 \varepsilon} \gamma^{3}\right)$. Finally, replacing $3 \varepsilon$ by $\varepsilon$, the estimate 3.62 follows.

3.7. Consequence of quasi-free approximation. The main consequence of the quasi-free approximation for the torus is Proposition 3.17 below. In preparation, we need two elementary lemmas. The quasi-free approximation upper bound (3.24) and the lower bound (3.25) are slightly different in that the upper bound is summed over all possible particle numbers in every small tori while the lower bound contains only the term that the number of particle in every small tori is identically its mean. Due to the convexity of free energy, it is not difficult to show that the fluctuations of number of particles can be estimated and they will be of lower order. This is the content of the next two lemmas. Once this is achieved, the quasi-free approximation upper and lower bound match up to a lower order terms. This establishes the additivity of the free energy up to negligible errors except that the range of Yukawa interactions are different for the gas in the original torus and the smaller one. However, the scaling of the free energy is given in Lemma 3.7 and the error due to the change of Yukawa range is easy to estimate. Thus we obtain that the existence of the specific free energy with effective error estimate in Proposition 3.17 .

Lemma 3.15. Let

$$
h_{\alpha}(\mathbf{n})=2 \pi \gamma^{2}\left(n_{\alpha}-\bar{n}\right)^{2}-n_{\alpha} \zeta^{(\gamma)}\left(n_{\alpha}\right)-\frac{1}{2} n_{\alpha} \log n_{\alpha}-\left(\frac{1}{2}-\frac{1}{\beta}\right) n_{\alpha} \log b^{-2} .
$$


Then the quasi-free free energy with particle profile $\mathbf{n}$ defined in (3.23) can be written as

$$
F(\mathbf{n})=\frac{1}{\beta} \log \left(\begin{array}{l}
N \\
\mathbf{n}
\end{array}\right)+2 \pi \ell^{2} N^{2}+\sum_{\alpha} h_{\alpha}(\mathbf{n})-N \log \ell .
$$

Proof. From 3.23) and 3.19 , recall that $F(\mathbf{n})=\frac{1}{\beta} \log \left(\begin{array}{l}N \\ \mathbf{n}\end{array}\right)-\sum_{\alpha} T_{\alpha}\left(n_{\alpha}\right)$, where

$$
T_{\alpha}\left(n_{\alpha}\right):=-\frac{1}{\beta} \log \int_{\mathbb{T}_{\alpha}^{n_{\alpha}}} \mathrm{e}^{-\beta \sum_{j \neq k} U_{\alpha}^{\ell}\left(z_{j}-z_{k}\right)} m(\mathrm{~d} \mathbf{z})=2 \pi \gamma^{2} n_{\alpha}^{2}-n_{\alpha} \log \ell-\xi_{b}^{(\gamma)}\left(n_{\alpha}\right) .
$$

By the scaling relation (3.21), we also have $h_{\alpha}(\mathbf{n})=2 \pi \gamma^{2}\left(n_{\alpha}-\bar{n}\right)^{2}-\xi_{b}^{(\gamma)}\left(n_{\alpha}\right)$. The equality

$$
\sum_{\alpha} 2 \pi \gamma^{2} n_{\alpha}^{2}=2 \pi \gamma^{2} \sum_{\alpha}\left(n_{\alpha}-\bar{n}\right)^{2}+2 \pi \ell^{2} N^{2}
$$

therefore implies

$$
\sum_{\alpha} T_{\alpha}\left(n_{\alpha}\right)=2 \pi \ell^{2} N^{2}+\sum_{\alpha} h_{\alpha}(\mathbf{n})-N \log \ell
$$

This completes the proof.

Lemma 3.16. For any functions $\mathcal{E}_{\alpha}: \mathbb{N} \rightarrow \mathbb{R}$ satisfying $\left|\mathcal{E}_{\alpha}(n)-\mathcal{E}_{\alpha}(m)\right| \leqslant \mathrm{O}\left(|n-m|(n+m)^{\varepsilon}\right)$, with $\gamma=\ell / b \geqslant N^{-C}$, we have

$$
\frac{1}{\beta} \log \sum_{\mathbf{n}} \mathrm{e}^{\beta \mathcal{E}(\mathbf{n})} \leq \mathcal{E}(\overline{\mathbf{n}})+N^{\mathrm{O}(\varepsilon)} \mathrm{O}\left(\ell^{-2}\right), \quad \mathcal{E}(\mathbf{n}):=\sum_{\alpha}\left[-2 \pi \gamma^{2}\left(n_{\alpha}-\bar{n}_{\alpha}\right)^{2}+\mathcal{E}_{\alpha}\left(n_{\alpha}\right)\right]
$$

Proof. By definition,

$$
\begin{aligned}
\frac{1}{\beta} \log \sum_{\mathbf{n}} \mathrm{e}^{\beta \mathcal{E}(\mathbf{n})}-\mathcal{E}(\overline{\mathbf{n}}) & =\frac{1}{\beta} \log \sum_{\mathbf{n}} \mathrm{e}^{\beta(\mathcal{E}(\mathbf{n})-\mathcal{E}(\overline{\mathbf{n}}))} \\
= & \frac{1}{\beta} \log \sum_{\mathbf{n}} \exp \left[\sum_{\alpha} \beta\left[-2 \pi \gamma^{2}\left(n_{\alpha}-\bar{n}_{\alpha}\right)^{2}+\left(\mathcal{E}_{\alpha}\left(n_{\alpha}\right)-\mathcal{E}_{\alpha}\left(\bar{n}_{\alpha}\right)\right]\right] .\right.
\end{aligned}
$$

To get an upper bound, we drop the constraint $\sum_{\alpha} n_{\alpha}=N$ on $\mathbf{n}$, and sum each $n_{\alpha}$ independently. Using the assumption $\left|\mathcal{E}_{\alpha}(n)-\mathcal{E}_{\alpha}(m)\right| \leq \mathrm{O}\left(|n-m|(n+m)^{\varepsilon}\right)$, the elementary inequality that for any positive fixed numbers $C, c>0$ and all integers $m \geq 0$,

$$
\sum_{n=0}^{\infty} \exp \left[C|n-m|(n+m)^{\varepsilon}-c \gamma^{2}(n-m)^{2}\right] \leq \mathrm{O}\left(\gamma^{-1}\right)\left(m+\gamma^{-2}\right)^{2 \varepsilon} \mathrm{e}^{\mathrm{O}\left(\gamma^{-2}\right)\left(m+\gamma^{-2}\right)^{2 \varepsilon}},
$$

and that $\bar{n}_{\alpha}=N b^{2}$, the left-hand side of 3.67 is bounded by

$$
\mathrm{O}(\log N) \sum_{\alpha} \gamma^{-2}\left(\bar{n}_{\alpha}+\gamma^{-2}\right)^{2 \varepsilon} \leq N^{\mathrm{O}(\varepsilon)} \mathrm{O}\left(\ell^{-2}\right)
$$

This completes the proof of the lemma.

Proposition 3.17. For any $\sigma>0$, there is $\tau>0$ such that if $\ell \geqslant N^{-1 / 2+\sigma}$ and $1 \geqslant b \geqslant N^{1+\sigma} \ell^{3}$,

$$
\zeta^{(\ell)}(N)=\zeta^{(\ell / b)}\left(N b^{2}\right)+\mathrm{O}\left(N^{-\tau}\right)
$$

More precisely, $\mathrm{O}\left(N^{-\tau}\right)$ is $N^{\varepsilon} \mathrm{O}\left(N \ell^{3} / b+1 /\left(N \ell^{2}\right)\right)$. 
Proof. The assumptions on $\ell$ and $b$ imply that the error terms in (3.24), 3.25) are $\mathrm{O}\left(N^{1-\tau}\right)$. By Propositions 3.8, 3.9, together with Lemma 3.15, therefore

$$
\begin{aligned}
& \frac{1}{\beta} \log \int \mathrm{e}^{-\beta H^{\ell}(\mathbf{z})} m(\mathrm{~d} \mathbf{z}) \geqslant-2 \pi \ell^{2} N^{2}+N \log \ell+\frac{1}{\beta} \log \left(\begin{array}{c}
N \\
\overline{\mathbf{n}}
\end{array}\right) \mathrm{e}^{-\beta \sum_{\alpha} h_{\alpha}(\overline{\mathbf{n}})}-\mathrm{O}\left(N^{1-\tau}\right), \\
& \frac{1}{\beta} \log \int \mathrm{e}^{-\beta H^{\ell}(\mathbf{z})} m(\mathrm{~d} \mathbf{z}) \leqslant-2 \pi \ell^{2} N^{2}+N \log \ell+\frac{1}{\beta} \log \sum_{\mathbf{n}}\left(\begin{array}{c}
N \\
\mathbf{n}
\end{array}\right) \mathrm{e}^{-\beta \sum_{\alpha} h_{\alpha}(\mathbf{n})}+\mathrm{O}\left(N^{1-\tau}\right) .
\end{aligned}
$$

We compute the sums on the right-hand sides of (3.70), (3.71). By Stirling's formula,

$$
\log \left(\begin{array}{l}
N \\
\mathbf{n}
\end{array}\right)=N \log N-\sum_{\alpha} n_{\alpha} \log n_{\alpha}+\mathrm{O}(\log N) .
$$

With the definition of $h$ from (3.64), and $\mathcal{E}_{\alpha}\left(n_{\alpha}\right)=\left(\frac{1}{2}-\frac{1}{\beta}\right) n_{\alpha} \log \left(n_{\alpha} b^{-2}\right)+n_{\alpha} \zeta^{(\gamma)}\left(n_{\alpha}\right)$ and $\mathcal{E}$ of (3.66), we rewrite (3.70), 3.71) as

$$
\begin{aligned}
& \frac{1}{\beta} \log \int \mathrm{e}^{-\beta H^{\ell}(\mathbf{z})} m(\mathrm{~d} \mathbf{z})+2 \pi \ell^{2} N^{2}-N \log \ell \geqslant \mathcal{E}(\overline{\mathbf{n}})+\frac{1}{\beta} N \log N+\mathrm{O}\left(N^{1-\tau}\right), \\
& \frac{1}{\beta} \log \int \mathrm{e}^{-\beta H^{\ell}(\mathbf{z})} m(\mathrm{~d} \mathbf{z})+2 \pi \ell^{2} N^{2}-N \log \ell \leqslant \frac{1}{\beta} \log \sum_{\mathbf{n}} \mathrm{e}^{\beta \mathcal{E}(\mathbf{n})}+\frac{1}{\beta} N \log N+\mathrm{O}\left(N^{1-\tau}\right) .
\end{aligned}
$$

By Lemma 3.5, $\mathcal{E}_{\alpha}$ satisfies the assumption of Lemma 3.16. Lemma 3.16 then shows that the sum over $\mathbf{n}$ can be estimated by its dominant term $\overline{\mathbf{n}}$ with error $N^{\varepsilon} \mathrm{O}\left(\ell^{-2}\right)=\mathrm{O}\left(N^{1-\tau}\right)$. Since

$$
\mathcal{E}(\overline{\mathbf{n}})=\left(\frac{1}{2}-\frac{1}{\beta}\right) \sum_{\alpha} \bar{n} \log \left(\bar{n} b^{-2}\right)+\sum_{\alpha} \bar{n} \zeta^{(\gamma)}(\bar{n})=\left(\frac{1}{2}-\frac{1}{\beta}\right) N \log N+N \zeta^{(\gamma)}\left(N b^{2}\right),
$$

this replacement yields

$$
\frac{1}{\beta} \log \int \mathrm{e}^{-\beta H_{V}^{\ell}(\mathbf{z})} m(\mathrm{~d} \mathbf{z})+2 \pi \ell^{2} N^{2}-N \log \ell=\frac{1}{2} N \log N+N \zeta^{(\gamma)}\left(N b^{2}\right)+\mathrm{O}\left(N^{1-\tau}\right),
$$

which completes the proof of 3.69 .

3.8. Existence of torus residual free energy: proof of Theorem 3.1. We now prove Theorem 3.1 The main ingredient is the next lemma which combines the previously proven estimates.

Lemma 3.18. For any $\sigma>0$ there exists $\tau>0$ such that for $\nu$ with $n^{-1 / 2+\sigma} \leqslant \nu \leqslant n^{-1 / 3-\sigma}$,

$$
\max _{\tilde{n} \in[n, 2 n]}\left|\zeta^{(\nu)}(n)-\zeta^{(\nu)}(\tilde{n})\right|=\mathrm{O}\left(n^{-\tau}\right) .
$$

More precisely, $\mathrm{O}\left(n^{-\tau}\right)$ is $n^{\varepsilon} \mathrm{O}\left(n \nu^{3}+1 /(\nu \sqrt{n})\right)$.

Proof. We will find $u_{0}(\sigma)>0$ such that the following statements hold. Given $n \in \mathbb{N}$, let $\tilde{n} \in[n, 2 n] \cap \mathbb{N}$. Choose $0 \leqslant u, \tilde{u} \leqslant u_{0}(\sigma)$ such that $B=n^{u}$ and $\tilde{B}=\tilde{n}^{\tilde{u}}$ are both integers and that $|\tilde{B} \sqrt{M}-B| \leqslant 1$ where $M=\tilde{n} / n \in[1,2]$. We also set $\ell=n^{-u} \nu$ and $\tilde{\ell}=\tilde{n}^{-\tilde{u}} \nu$. We claim that the following statements hold:

$$
\begin{aligned}
& \zeta^{(\ell)}\left(B^{2} n\right)=\zeta^{(\nu)}(n)+\mathrm{O}\left(n^{-\tau}\right), \\
& \zeta^{(\tilde{\ell})}\left(\tilde{B}^{2} \tilde{n}\right)=\zeta^{(\nu)}(\tilde{n})+\mathrm{O}\left(n^{-\tau}\right), \\
& \zeta^{(\tilde{\ell})}\left(\tilde{B}^{2} \tilde{n}\right)-\zeta^{(\ell)}\left(\tilde{B}^{2} \tilde{n}\right)=\mathrm{O}\left(n^{-\tau}\right), \\
& \zeta^{(\ell)}\left(B^{2} n\right)-\zeta^{(\ell)}\left(\tilde{B}^{2} \tilde{n}\right)=\mathrm{O}\left(n^{-\tau}\right) .
\end{aligned}
$$


By combining the estimates (3.74), 3.75, 3.76), (3.77), we obtain (3.73).

To prove (3.74), we apply Proposition 3.17 with $N=B^{2} n$ and $b=1 / B$. For $u$ sufficiently small, the assumptions of this lemma imply that the assumptions of Proposition 3.17 are satisfied. Thus the resulting error estimate of Proposition 3.17 becomes $N^{\varepsilon} \mathrm{O}\left(N \ell^{3} / b+1 /\left(N \ell^{2}\right)\right)=$ $n^{2 \varepsilon} \mathrm{O}\left(n^{1-2 u} \nu^{3}+1 /\left(n \nu^{2}\right)\right) \leqslant n^{2 \varepsilon} \mathrm{O}\left(n^{1-2 u} \nu^{3}+1 /\left(n^{1 / 2+\sigma} \nu\right)\right)=\mathrm{O}\left(n^{-\tau}\right)$. This completes the proof of (3.74). The proof of (3.75) can be done analogously.

To prove (3.76), we apply $(3.4)$ with $N=\tilde{B}^{2} \tilde{n}$. This gives the needed bound since $1 /\left(N \ell^{2}\right) \leqslant$ $n^{2(u-\tilde{u})-1} \nu^{-2}=\mathrm{O}\left(n^{2(u-\tilde{u})-\sigma} /(\sqrt{n} \nu)\right)=\mathrm{O}(1 /(\sqrt{n} \nu))$ when $u$ and $\tilde{u}$ are small depending on $\sigma$.

To prove (3.77), we apply (3.13). Since $\left|\tilde{B}^{2} M-B^{2}\right| \leq \mathrm{O}(B)$, (3.13) implies

$$
\left|\zeta^{(\ell)}\left(B^{2} n\right)-\zeta^{(\ell)}\left(\tilde{B}^{2} \tilde{n}\right)\right|=\mathrm{O}\left(\frac{\left|\tilde{B}^{2} \tilde{n}-B^{2} n\right|}{\left|\tilde{B}^{2} \tilde{n}+B^{2} n\right|^{1-\varepsilon}}\right)=\mathrm{O}\left(\frac{\left|\tilde{B}^{2} M-B^{2}\right| n^{\varepsilon}}{\left|\tilde{B}^{2} M+B^{2}\right|^{1-\varepsilon}}\right) \leq \frac{\mathrm{O}\left(n^{\varepsilon}\right)}{B^{1-2 \varepsilon}}=\mathrm{O}\left(n^{-\tau}\right),
$$

where the last inequality follows from $n^{\varepsilon} \mathrm{O}(1 / B)=n^{\varepsilon} \mathrm{O}(1 /(\sqrt{n} \nu))=\mathrm{O}\left(n^{-\tau}\right)$.

Proof of Theorem 3.1. Recall $\zeta$ is defined in (3.2). We set a constant $c=3 / 8$ and for $j \in \mathbb{N}$, define the sequences $n_{j}=2^{j}, \nu_{j}=2^{-c j}$ and $\zeta_{j}=\zeta^{\left(\nu_{j}\right)}\left(n_{j}\right)$. For $k \geqslant i$, we then write

$$
\left|\zeta_{i}-\zeta_{k}\right| \leqslant \sum_{j=i}^{k-1}\left|\zeta^{\left(\nu_{j}\right)}\left(n_{j}\right)-\zeta^{\left(\nu_{j}\right)}\left(n_{j+1}\right)\right|+\sum_{j=i}^{k-1}\left|\zeta^{\left(\nu_{j}\right)}\left(n_{j+1}\right)-\zeta^{\left(\nu_{j+1}\right)}\left(n_{j+1}\right)\right| .
$$

We estimate the first sum in $(3.79$ by using Lemma 3.18. Note that since $c=3 / 8 \in(1 / 3,1 / 2)$, the assumptions of Lemma 3.18 are satisfied with $n=n_{j}$ and $\nu=\nu_{j}$. Thus the first sum can be bounded by $\sum_{j=i}^{k-1} n_{j}^{\varepsilon} \mathrm{O}\left(n_{j} \nu_{j}^{3}+1 /\left(\nu_{j} \sqrt{n_{j}}\right)\right)=\mathrm{O}\left(2^{-(1 / 8-\varepsilon) i}\right)$. For the second sum in 3.79), we use (3.4) and obtain the estimate $\sum_{j=i}^{k-1} \mathrm{O}\left(n_{j}^{-1+\varepsilon} \nu_{j}^{-2}\right)=\mathrm{O}\left(2^{-(1 / 4-\varepsilon) i}\right)$.

In summary, with $\tau=1 / 8-\varepsilon$, we have shown that $\zeta_{k}=\zeta_{i}+\mathrm{O}\left(2^{-i \tau}\right)$ for $k \geqslant i$ sufficiently

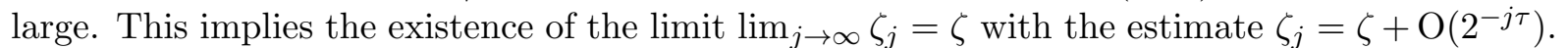

Finally, it remains to pass from the limit along the dyadic sequence above to that for general $N$ and $\ell$ as in the statement of the theorem. Given $N$ large, we let $j_{N}$ be the smallest integer $j$ such that $2^{j} \geqslant N$. By (3.4) respectively (3.73), then

$$
\begin{aligned}
\zeta^{(\ell)}(N) & =\zeta^{\left(\nu_{j_{N}}\right)}(N)+\mathrm{O}\left(N^{-2 \sigma+\varepsilon}\right), \\
\zeta^{\left(\nu_{j_{N}}\right)}(N) & =\zeta_{j_{N}}+\mathrm{O}\left(N^{-1 / 8+\varepsilon}\right) .
\end{aligned}
$$

Combining these two inequalities then gives the claim with $N^{\varepsilon} \mathrm{O}\left(N^{-1 / 8}+N^{-2 \sigma}\right)=\mathrm{O}\left(N^{-\kappa}\right)$.

\section{Proof of Theorem 1.1: quasi-free approximation of free energy}

In this and the following three sections, we prove Theorem 1.1 and its local version Theorem 4.2 . The proofs of both theorems will be parallel and we will give detailed arguments for the proof of Theorem 1.1 and remark along the way the modifications needed for the proof of its local version.

We follow the strategy of quasi-free approximation analogously as for the torus in Section 3 . The main differences are that the equilibrium measure can have a non-constant density and that its support has a boundary. In this section, we present the set-up of the quasi-free approximation, and give the proof of Theorem 1.1 assuming using Propositions 4.54 .6 which are proved subsequently in Sections 5 7. In Section 5, we prove the upper bound on the partition function. As in the torus case, this upper bound can essentially be established using the Jensen inequality 
and the positive definiteness of the Coulomb potential. In Section 7, we prove the lower bound. The lower bound involves estimating the Coulomb energy near the boundary of the support of the equilibrium measure and is the main difficulty of the proof.

4.1. Main result. We recall the definition of the two-dimensional Yukawa gas with range $R$ and external potential $V$ as well as the related potential theory from Section 2, In particular,

$$
H_{V}^{R}(\mathbf{z})=N \sum_{j} V\left(z_{j}\right)+\sum_{j \neq k} Y^{R}\left(z_{j}-z_{k}\right)
$$

is the corresponding Hamiltonian, $\mu_{V}^{R}$ is the equilibrium measure, $\rho_{V}^{R}$ denotes the density of its absolutely continuous part, and $I_{V}^{R}$ is the minimizing energy of the variational functional.

Theorem 4.1. Assume that $V$ satisfies the assumptions of Theorem 1.1 or more generally those stated in Theorem 4.2 below. Then for any $\sigma>0$, there exists a constant $\kappa>0$ such that, for all $R \geqslant N^{2}$,

$\frac{1}{\beta N} \log \int_{\mathbb{C}^{N}} \mathrm{e}^{-\beta H_{V}^{R}(\mathbf{z})} m(\mathrm{~d} \mathbf{z})=-N I_{V}^{R}+\log R+\frac{1}{2} \log N+\zeta+\left(\frac{1}{2}-\frac{1}{\beta}\right) \int_{\mathbb{C}} \rho_{V}^{R} \log \rho_{V}^{R} \mathrm{~d} m+\mathrm{O}\left(N^{-\kappa}\right)$,

where $\zeta$ is the residual torus free energy of Theorem 3.1. For $R \geqslant 1$, any $\kappa<1 / 24$ is admissible.

The remainder of Section 4 is devoted to the proof of Theorem 4.1, which is concluded in Section 4.5, subject to the proofs of Propositions 4.5 4.6, which will be proved in Sections 5 and 7. Theorem 1.1 for the Coulomb gas is then a direct consequence, by taking $R \rightarrow \infty$, which we do in detail in Section 4.6.

Throughout this section, we make the standing assumptions $R \geqslant N^{2}$, and that $V$ satisfies the assumptions of Theorem 1.1, i.e., the asymptotic condition $(1.4)$ and $(1.10)$, or more generally that the conditions of the remark below hold. We denote the empirical measure by $\hat{\mu}$ and its difference with equilibrium measure by $\tilde{\mu}=\tilde{\mu}_{V}^{R}$, i.e.,

$$
\hat{\mu}=N^{-1} \sum_{j} \delta_{z_{j}}, \quad \tilde{\mu}=\tilde{\mu}_{V}^{R}=\hat{\mu}-\mu_{V}^{R}
$$

We denote the expectation of the Coulomb gas with density $\mathrm{e}^{-\beta H_{V}^{R}}$ by $\mathbb{E}_{V}^{R}$. The following Theorem is a local version of Theorem 1.1. A more precise statement with precise scaling and notation will be given in Theorem 8.15. We choose to present it in the following way so that it is easier to digest in the first reading.

Theorem 4.2 (A local version of Theorem 1.1). Consider the setting of Theorem 1.1. Then the good boundary conditions hold with high probability. Furthermore, Theorem 1.1 hold with respect to the conditional measure with the error term $\mathrm{O}\left(N^{-\kappa}\right)$ replaced by

$$
C(\Omega, A)\left(1+K^{2}\right)\left(N r^{2}\right)^{-\left(\kappa \wedge a^{\prime}\right)}
$$

for any $a^{\prime}<a$, where $a$ is the constant in 2.32 .

4.2. Short-range Yukawa approximation. The first step is a decomposition of the Yukawa potential into a short-range and a long-range part. This is similar to our strategy in the proof of Lemma 3.3 for the torus. However, due to the presence of a boundary of the support of the equilibrium measure and lack of rigidity estimates there, we cannot prove an analog of Lemma 3.3 . Therefore we subsequently cannot drop the long-range part of the interaction near the boundary. 
Given $0<\ell<R$, we decompose the Yukawa potential as $Y^{R}=Y^{\ell}(z)+L_{R}^{\ell}(z)$. The formula (2.1) shows that the Fourier transform of $L_{R}^{\ell}$ is positive and thus that $L_{R}^{\ell}$ is a positive definite function on $\mathbb{C}$. The next lemma expresses the long-range contribution to the interaction in terms of a effective potential $Q$ and an error. We set

$$
\begin{array}{r}
Q(z)=Q_{R}^{\ell}(z)=V(z)+2 \int L_{R}^{\ell}(z-w) \mu_{V}^{R}(\mathrm{~d} w), \\
\mathbf{L}=\mathbf{L}_{R}^{\ell}=\int L_{R}^{\ell}(z-w) \tilde{\mu}_{V}^{R}(\mathrm{~d} w) \tilde{\mu}_{V}^{R}(\mathrm{~d} z), \\
K=K_{R}^{\ell}=\int L_{R}^{\ell}(z-w) \mu_{V}^{R}(\mathrm{~d} w) \mu_{V}^{R}(\mathrm{~d} z),
\end{array}
$$

where $K_{R}^{\ell}$ is the equilibrium interaction energy of the potential difference $L_{R}^{\ell}=Y^{R}-Y^{\ell}$.

Lemma 4.3. Let $0<\ell<R$, and let $Q, \mathbf{L}$, and $K$ be as above. Then we have the identity

$$
\sum_{j \neq k} L_{R}^{\ell}\left(z_{j}-z_{k}\right)+N \sum_{j} V\left(z_{j}\right)=N \sum_{j} Q_{R}^{\ell}\left(z_{j}\right)+N^{2} \mathbf{L}_{R}^{\ell}-N \log (R / \ell)-N^{2} K_{R}^{\ell}
$$

and in particular

$$
H_{Q}^{\ell}(\mathbf{z})=H_{V}^{R}(\mathbf{z})-N^{2} \mathbf{L}_{R}^{\ell}+N \log (R / \ell)+N^{2} K_{R}^{\ell}
$$

Moreover, the minimizers of the variational functionals $\mathcal{I}_{Q}^{\ell}$ and $\mathcal{I}_{V}^{R}$ coincide, i.e., $\mu_{Q}^{\ell}=\mu_{V}^{R}$, and their energies satisfy $I_{Q}^{\ell}=I_{V}^{R}+K_{R}^{\ell}$. The Euler-Lagrange equation for the measure $\mu_{Q}^{\ell}$ is

$$
\begin{array}{ll}
\int Y^{\ell}(z-w) \mu_{Q}^{\ell}(\mathrm{d} w)+\frac{1}{2} Q(z)=c_{V} & \text { q.e. in } S_{V}^{R} \quad \text { and } \\
\int Y^{\ell}(z-w) \mu_{Q}^{\ell}(\mathrm{d} w)+\frac{1}{2} Q(z) \geqslant c_{V} & \text { q.e. in } \mathbb{C},
\end{array}
$$

with the same constant $c_{V}$ as in the Euler-Lagrange equation for $\mu_{V}^{R}$.

Proof. The proof of 4.6$)$ is a direct calculation. Indeed, using that $L_{R}^{\ell}(0)=\log (R / \ell)$ by $(2.2)$, it follows that

$$
\int_{z \neq w} L_{R}^{\ell}(z-w) \tilde{\mu}_{V}^{R}(\mathrm{~d} w) \tilde{\mu}_{V}^{R}(\mathrm{~d} z)=\int L_{R}^{\ell}(z-w) \tilde{\mu}_{V}^{R}(\mathrm{~d} w) \tilde{\mu}_{V}^{R}(\mathrm{~d} z)-\frac{1}{N} \log (R / \ell) .
$$

The equilibrium measures (minimizers) of $\mathcal{I}_{V}^{R}$ and $\mathcal{I}_{Q}^{\ell}$ are characterized by the Euler-Lagrange equations (2.13), which state that in the supports of the measures, the equalities

$$
\frac{1}{2} V+Y^{R} * \mu_{V}^{R}=c_{V}^{R}, \quad \frac{1}{2} Q+Y^{\ell} * \mu_{Q}^{\ell}=c_{Q}^{\ell}
$$

hold, and that equality is replaced by inequality outside the supports of the equilibrium measures. By definition of $Q$ and the Euler-Lagrange equation for $\mu_{V}^{R}$, the solution $\mu_{Q}^{\ell}$ satisfies (4.8). By the uniqueness of the minimizers, we thus conclude that $\mu_{Q}^{\ell}=\mu_{V}^{R}$ and $S_{V}^{R}=S_{Q}^{\ell}$, i.e., the two minimizers coincide. Moreover, a simple computation yields that $I_{V}^{R}=I_{Q}^{\ell}+K_{R}^{\ell}$.

In view of the above lemma, we write $\mu_{V}$ instead of $\mu_{V}^{R}=\mu_{Q}^{\ell}$ from now on, we write $\rho_{V}$ for the density of the absolutely continuous part of $\mu_{V}$, and $S_{V}$ for its support. The next lemma gives an elementary estimate on $Q$ that will be useful later. 
Lemma 4.4. For $z \in S_{V}$ with distance $\gg \ell$ to the complement of $S_{V}$,

$$
Q(z)=2 c_{V}-4 \pi \ell^{2} \rho_{V}(z)+N^{\varepsilon} \mathrm{O}\left(\ell^{4}\right)\left\|\nabla^{2} \rho_{V}\right\|_{\infty}+\mathrm{O}\left(N^{-\infty}\right) .
$$

Proof. By Lemma 4.3, for $z \in S_{V}$, we have

$$
\begin{aligned}
Q(z) & =2 c_{V}-2 \int Y^{\ell}(z-w) \mu_{V}(\mathrm{~d} w) \\
& =2 c_{V}-2 \rho_{V}(z) \int Y^{\ell}(z-w) m(\mathrm{~d} w)+N^{\varepsilon} \mathrm{O}\left(\ell^{4}\right)\left\|\nabla^{2} \rho_{V}\right\|_{\infty}+\mathrm{O}\left(N^{-\infty}\right) .
\end{aligned}
$$

In the second equality, we used that, by the exponential decay of $Y^{\ell}$, we may restrict the integral over $w$ a disk of radius $\mathrm{O}\left(\ell N^{\varepsilon}\right)$ around $z$, up to an error $\mathrm{O}\left(N^{-\infty}\right)$. Moreover, since $z$ is in the support of the absolutely continuous part of $\mu_{V}$ with distance $\gg \ell$ to its complement, we may Taylor expand the equilibrium density to second order and use that the first-order term vanishes after integration. The definition of the Yukawa potential (2.1) implies $\int Y^{\ell}(z-w) m(\mathrm{~d} w)=2 \pi \ell^{2}$. This implies 4.9 .

4.3. Quasi-free approximation. In this and the next subsections, we approximate the partition function of the (long-range) Yukawa gas in terms of the quasi-free Yukawa approximation, which we now define. The idea is the same as in Section 3.4, with the additional element that now the boundary requires a special treatment.

Given parameters which we will chose later on with the constraint

$$
N^{-1 / 2+\sigma} \ll \ell \ll b \leqslant b^{\prime} \ll 1, \quad \ell<R,
$$

we divide $\mathbb{C}$ into a grid of squares $\alpha$ of side length $b$ with centers $c(\alpha) \in(b \mathbb{Z})^{2} \subset \mathbb{C}$. The last constraint 4.10 will be assumed through out this paper. It will also be useful to also consider the shifted grid, in which all squares are translated by $u \in[-b / 2, b / 2)^{2}$ so that their centers are $u+c(\alpha)$. We write $\mathbf{S}_{u}$ for the set of squares partitioning $\mathbb{C}$ such that the square containing 0 has $u$ as its center. We say that the square $\alpha \in \mathbf{S}_{0}$ is in the bulk if it and its translates by $u \in[-b / 2, b / 2)^{2}$ have distance at least $b^{\prime}$ to the complement of $S_{V}$ (respectively $\Omega$ in the situation of Theorem 4.2). Denote by $D_{0}$ the union of the bulk squares in $\mathbf{S}_{0}$ and by $B_{0}=S_{V} \backslash D_{0}$ the remaining boundary region. For a square $\alpha \in \mathbf{S}_{u}$ we define that $\alpha$ is in the bulk if $\alpha-u \in \mathbf{S}_{0}$ is the bulk. Similarly, we define $D_{u}$ the union of the bulk squares satisfying the previous condition and denote by $B_{u}=S_{V} \backslash D_{u}$ the boundary region in this case. We will use the notation $\alpha \in D_{u}$ (or $\alpha \subset D_{u}$ ) to denote that $\alpha$ is a bulk cube. Throughout Section 4 . we assume in addition to 4.10 the following condtion:

$$
b^{\prime} \gg N^{-1 / 4} \text {. }
$$

In the context of Theorem 4.2 , we assume that $b^{\prime} \geqslant N^{-a}$ instead of $b^{\prime} \gg N^{-1 / 4}$.

Given parameters as above, we consider the quasi-free Yukawa gas obtained by removing the interaction between particles in a bulk square with particles outside that square, and replacing the interactions between particles in the same square by a periodic one inside each bulk square. For the particles in the boundary region, we will use independent particle approximation with density given by the equilibrium density $\rho_{V}$ near the boundary. Since the boundary region $B$ has an area of smaller order when compared with the interior domain $D$, the independent particle approximation is already sufficient to approximate the $\log$ partition function to order $N^{1-c}$.

Fix $u \in[-b / 2, b / 2)^{2}$. The following definitions depend on $u$, but we do not make this explicit in the notation. Firstly, we write $\overline{\mathbf{S}}=\overline{\mathbf{S}}_{u}$ for the set

$$
\overline{\mathbf{S}}_{u}=\left\{u+\alpha: \alpha \in \mathbf{S}_{0}, \alpha \subset D_{u}\right\} \cup\left\{B_{u}\right\},
$$


i.e. the set of bulk squares together with the boundary region. Let $\mathbf{n}=\left(n_{\alpha}\right)$ be a particle profile with $\sum_{\alpha} n_{\alpha}=N$. Similarly as in (3.23), we define the quasi-free free energy for particle profile $\mathbf{n}$ by

$$
F(\mathbf{n})=\frac{1}{\beta} \log \left(\begin{array}{l}
N \\
\mathbf{n}
\end{array}\right)+\frac{1}{\beta} \sum_{\alpha \subset D} \log \int_{\mathbb{T}_{\alpha}^{n_{\alpha}}} \mathrm{e}^{-\beta \hat{H}_{\alpha}(\mathbf{v})} m(\mathrm{~d} \mathbf{v})-\hat{H}_{B},
$$

with

$$
\hat{H}_{\alpha}(\mathbf{v})=\sum_{i \neq j} U_{\alpha}^{\ell}\left(v_{i}-v_{j}\right)+N n_{\alpha} Q(\alpha), \quad \hat{H}_{B}=N^{2} I_{Q, B}+2 c_{V} N\left(n_{B}-N \mu_{V}(B)\right),
$$

where $Q(\alpha):=Q(c(\alpha))$ and $I_{Q, B}$ is a constant defined in (5.13) below. We denote by $\overline{\mathbf{n}}=\left(\bar{n}_{\alpha}\right)$ the approximate mean number of particles in $\alpha$, where $\alpha$ is either a square or the boundary region. More precisely, we choose $\bar{n}_{\alpha}$ to be an integer at distance at most 1 to $N \mu_{V}(\alpha)$; we assume that this rounded choice is such that $\sum_{\alpha} \bar{n}_{\alpha}=N$. The precise choice of $\bar{n}_{\alpha}$ is not important as long as it is within 1 distance to $n_{\alpha}$ and $\sum_{\alpha} \bar{n}_{\alpha}=N$. We also impose the convention that sums over $\mathbf{n}$ will always be over all particle profiles with $\sum_{\alpha} n_{\alpha}=N$.

We will prove the following upper and lower bounds on the partition function in terms of the quasifree free energy.

Proposition 4.5 (Upper Bound). Assume that the parameters $b, b^{\prime}$ satisfy (4.10) and (4.11). Then there exists $u \in[-b / 2, b / 2)^{2}$ such that

$$
\begin{aligned}
\frac{1}{\beta} \log \int \mathrm{e}^{-\beta H_{V}^{R}(\mathbf{z})} m(\mathrm{~d} \mathbf{z})-N \log (R / \ell)- & N^{2} K_{R}^{\ell} \leqslant \frac{1}{\beta} \log \sum_{\mathbf{n}} \mathrm{e}^{\beta F(\mathbf{n})} \\
& +N^{\varepsilon} \mathrm{O}\left(N^{2} \ell^{3} b^{-1}+N^{2} \ell^{2} b\right)\left\|\rho_{V}\right\|_{\infty, 2}+\mathrm{O}\left(n_{B} \log N\right),
\end{aligned}
$$

where $\left\|\rho_{V}\right\|_{\infty, 2}$ is defined in 1.7 .

The error terms in 4.15) can be understood as follows. The error $N^{2} \ell^{3} b^{-1}=\left(N \ell^{2}\right)\left(N^{2} \ell b^{-1}\right)$ is the number of pair interactions via a Yukawa gas of range $\ell$ for particles in neighboring squares; the error $N^{2} \ell^{2} b$ is the variation of the effective potential $Q$ over a square of size $b$. The error terms in the following lower bound cannot be obtained by a simple counting as the bound relies on higher order cancellations which we will explain later on.

Proposition 4.6 (Lower Bound). Assume $N^{-1 / 2+\sigma} \ll \ell \ll b \ll N^{-2 c / 5}$ for some small $c>0$, $\ell<R$, and $1 \gg \ell / b \gg\left(N b^{2}\right)^{-1 / 4}$. Then, with $\tau=2 \sigma / 5$, for all $u \in[-b / 2, b / 2)^{2}$,

$$
\begin{aligned}
& \frac{1}{\beta} \log \int \mathrm{e}^{-\beta H_{V}^{R}(\mathbf{z})} m(\mathrm{~d} \mathbf{z})-N \log (R / \ell)-N^{2} K_{R}^{\ell} \geq F(\overline{\mathbf{n}})+N^{\varepsilon} \mathrm{O}\left(N^{1-\tau}+b^{2} \ell^{-4}\right) \\
&+\mathrm{O}\left(N^{2}\left(b^{4}+\ell^{2} b\right)\right)\left(\left\|\rho_{V}\right\|_{\infty, 3}+\left\|\rho_{V}\right\|_{\infty, 3}^{2}\right)+\mathrm{O}\left(b^{-2} \log N+\bar{n}_{B} \log N\right) .
\end{aligned}
$$

More precisely, $\mathrm{O}\left(N^{1-\tau}\right)$ is $N^{\varepsilon} \mathrm{O}\left(N^{4 / 5} \ell^{-2 / 5}+N b\right)$.

Propositions 4.5 and 4.6 will be proved in Sections 5 7. In the remainder of Section 4, we complete the proof of Theorem 4.1 assuming these propositions. They assert that the free energy of a Yukawa gas with (long) range $R$ can be approximated by that of the quasi-free Yukawa gases with range $\ell \ll R$, for appropriate choices of the parameters $b, b^{\prime}$ and $\ell$. These propositions are analogous to Propositions 3.8 and 3.9 , with the additional treatment of the boundary and taking into account that the density of the equilibrium measure is in general not constant.

We end this subsection by recording the following simple estimates for the bulk and boundary regions. In the following, we usually omit the parameter $u$ from $D_{u}$ (the union of bulk squares) and write $B=S_{V} \backslash D$ to denote the boundary region. We write $\bigcap D=\bigcap_{u} D_{u}$ and $\bigcup D=\bigcup_{u} D_{u}$. 
Lemma 4.7. The following bounds hold uniformly in the shift parameter $u \in[-b / 2, b / 2)^{2}$. The number of bulk squares (which is independent of $u$ ) is $\mathrm{O}\left(b^{-2}\right)$, the number of bulk squares touching the boundary region is $\mathrm{O}\left(b^{-1}\right)$, and the equilibrium mass covered by the bulk squares is $\mu_{V}(\cap D) \geqslant$ $1-\mathrm{O}\left(b^{\prime}\right)$. In addition, for any $\alpha \subset D$,

$$
\bar{n}_{\alpha}=\mathrm{O}\left(N b^{2}\right)\left\|\rho_{V}\right\|_{\infty}, \quad \bar{n}_{\alpha}=N b^{2} \rho(\alpha)+\mathrm{O}\left(N b^{3}\right)\left\|\nabla \rho_{V}\right\|_{\infty}, \quad \bar{n}_{B}=\mathrm{O}\left(N b^{\prime}\right) .
$$

Proof. The claim about the number of bulk squares follows immediately from the fact that the support of $S_{V}$ has diameter of order 1 . The statements about the number of squares touching the boundary region and the mass not covered by the squares follow from the assumption that the the boundary of $S_{V}$ is piecewise $C^{1}$. In the more general situation of Theorem 4.2 , the estimates hold by the assumption stated in the remark. Finally, 4.17) follows immediately from the fact that, by construction, $\rho_{V}$ is $C^{1}$ on the squares $\alpha$.

4.4. Consequence of quasi-free approximation. With the upper and lower bounds established in Propositions 4.5 and 4.6 , the remainder of the proof is similar to that for the torus. In fact, the following proof is simpler since the limit of the torus free energy has already been established.

First, analogously to (3.64), we define

$$
h_{\alpha}(\mathbf{n})=2 \pi \gamma^{2}\left(n_{\alpha}-\bar{n}_{\alpha}\right)^{2}-n_{\alpha} \zeta-\frac{1}{2} n_{\alpha} \log n_{\alpha}-\left(\frac{1}{2}-\frac{1}{\beta}\right) n_{\alpha} \log b^{-2}, \quad \text { where } \gamma=\ell / b .
$$

Then, similarly to Lemma 3.15 , we have the following estimate for $F(\mathbf{n})$ defined in 4.13 .

Lemma 4.8. Assume that $b$ satisfies (4.10). There exists $\tau>0$ such that

$F(\mathbf{n})+N \log \ell-N^{2} K_{R}^{\ell}-N^{2} I_{V}^{R}=\frac{1}{\beta} \log \left(\begin{array}{l}N \\ \mathbf{n}\end{array}\right)-\sum_{\alpha \subset D} h_{\alpha}(\mathbf{n})+\mathrm{O}\left(N^{2} \ell^{2} b\right)\left(1+\left\|\rho_{V}\right\|_{\infty, 1}\right)^{2}+\mathrm{O}\left(N^{1-\tau}\right)$

where $\left\|\rho_{V}\right\|_{\infty, 1}$ is defined in 1.7). More precisely, the error $\mathrm{O}\left(N^{1-\tau}\right)$ is $N^{\varepsilon} \mathrm{O}\left(N^{7 / 8} / b^{1 / 4}+\ell^{-2}\right)$.

Proof. From (4.13), recall that

$$
F(\mathbf{n})=\frac{1}{\beta} \log \left(\begin{array}{l}
N \\
\mathbf{n}
\end{array}\right)-\sum_{\alpha} T_{\alpha}\left(n_{\alpha}\right)-\hat{H}_{B}, \quad T_{\alpha}\left(n_{\alpha}\right):=-\frac{1}{\beta} \log \int_{\mathbb{T}_{\alpha}^{n_{\alpha}}} \mathrm{e}^{-\beta \hat{H}_{\alpha}(\mathbf{z})} m(\mathrm{~d} \mathbf{z}),
$$

where here and in the rest of this proof, all summations over $\alpha$ are over $\alpha \subset D$. Recall the defintion of $\hat{H}_{B}$ from (4.14), hence Lemma 4.8 follows from

$$
\begin{aligned}
\sum_{\alpha} T_{\alpha}\left(n_{\alpha}\right)+N^{2} I_{Q, B} & +2 c_{V} N\left(n_{B}-N \mu_{V}(B)\right)-N^{2} K_{R}^{\ell} \\
= & N^{2} I_{V}^{R}-N \log \ell+\sum_{\alpha \subset D} h_{\alpha}(\mathbf{n})+\mathrm{O}\left(N^{2} \ell^{2} b\left(1+\left\|\rho_{V}\right\|_{\infty, 1}\right)^{2}\right)+\mathrm{O}\left(N^{1-\tau}\right),
\end{aligned}
$$

which we now prove. By definition of $T_{\alpha}$,

$$
\begin{aligned}
T_{\alpha}\left(n_{\alpha}\right) & =N n_{\alpha} Q(\alpha)+2 \pi \gamma^{2} n_{\alpha}^{2}-n_{\alpha} \log \ell-\xi_{b}^{(\gamma)}\left(n_{\alpha}\right) \\
& =N n_{\alpha} Q(\alpha)+2 \pi \gamma^{2} n_{\alpha}^{2}-n_{\alpha} \log \ell-n_{\alpha} \zeta^{(\gamma)}\left(n_{\alpha}\right)-\frac{1}{2} n_{\alpha} \log n_{\alpha}-\left(\frac{1}{2}-\frac{1}{\beta}\right) n_{\alpha} \log b^{-2}
\end{aligned}
$$


By Theorem 3.1, $n_{\alpha} \zeta^{(\gamma)}\left(n_{\alpha}\right)=n_{\alpha} \zeta+N^{\varepsilon} \mathrm{O}\left(n_{\alpha}^{7 / 8}+1 / \gamma^{2}\right)$ so that $\sum_{\alpha} n_{\alpha} \zeta^{(\gamma)}\left(n_{\alpha}\right)=N \zeta+$ $b^{-2} N^{\varepsilon} \mathrm{O}\left(\left(N b^{2}\right)^{7 / 8}+(\ell / b)^{-2}\right)=N \zeta+N^{\varepsilon} \mathrm{O}\left(N^{7 / 8} / b^{1 / 4}+\ell^{-2}\right)$. Therefore

$$
\begin{aligned}
\sum_{\alpha} T_{\alpha}\left(n_{\alpha}\right)=\sum_{\alpha}\left(N n_{\alpha} Q(\alpha)+2 \pi \gamma^{2} n_{\alpha}^{2}-\frac{1}{2} n_{\alpha} \log n_{\alpha}-\left(\frac{1}{2}-\frac{1}{\beta}\right) n_{\alpha} \log b^{-2}\right) \\
-N \log \ell-N \zeta+N^{\varepsilon} \mathrm{O}\left(N^{7 / 8} / b^{1 / 4}+\ell^{-2}\right) .
\end{aligned}
$$

By definition of $h_{\alpha}(\mathbf{n})$ in 4.18 and since

$$
\sum_{\alpha} 2 \pi \gamma^{2} n_{\alpha}^{2}=2 \pi \gamma^{2} \sum_{\alpha}\left(n_{\alpha}-\bar{n}_{\alpha}\right)^{2}+4 \pi \gamma^{2} \sum_{\alpha} n_{\alpha} \bar{n}_{\alpha}-2 \pi \gamma^{2} \sum_{\alpha} \bar{n}_{\alpha}^{2}
$$

we obtain

$\sum_{\alpha} T_{\alpha}\left(n_{\alpha}\right)-\sum_{\alpha} h_{\alpha}\left(n_{\alpha}\right)+N \log \ell=\sum_{\alpha}\left(N n_{\alpha} Q(\alpha)+4 \pi \gamma^{2} n_{\alpha} \bar{n}_{\alpha}-2 \pi \gamma^{2} \bar{n}_{\alpha}^{2}\right)+\mathrm{O}\left(N^{\frac{7}{8}+\varepsilon} / b^{\frac{1}{4}}+\ell^{-2}\right)$.

We now compute the right-hand side of the last equation. Using that $\gamma=\ell / b$, that $2 \pi \ell^{2}=$ $\int Y^{\ell}(z) m(\mathrm{~d} z)=\int_{\alpha} Y^{\ell}(z) m(\mathrm{~d} z)+\mathrm{O}\left(N^{-\infty}\right)$, and that $\bar{n}_{\alpha}=N b^{2} \rho_{V}(z)+\mathrm{O}\left(N b^{3}\right)\left\|\nabla \rho_{V}\right\|_{\infty}=$ $N \int_{\alpha} \rho_{V}(w) m(\mathrm{~d} w)+\mathrm{O}\left(N b^{3}\right)\left\|\nabla \rho_{V}\right\|_{\infty}$ for any $z \in \alpha$, we obtain

$$
\begin{aligned}
2 \pi \gamma^{2} \sum_{\alpha} \bar{n}_{\alpha}^{2} & =N^{2} \sum_{\alpha} \iint_{D \times \alpha} Y^{\ell}(z-w) \rho_{V}(z) \rho_{V}(w) m(\mathrm{~d} z) m(\mathrm{~d} w)+\mathrm{O}\left(N^{2} \ell^{2} b\right)\left\|\rho_{V}\right\|_{\infty}\left\|\nabla \rho_{V}\right\|_{\infty} \\
& =N^{2} \iint_{D \times D} Y^{\ell}(z-w) \mu_{V}(\mathrm{~d} z) \mu_{V}(\mathrm{~d} w)+\mathrm{O}\left(N^{2} \ell^{2} b\right)\left\|\rho_{V}\right\|_{\infty}\left\|\nabla \rho_{V}\right\|_{\infty} .
\end{aligned}
$$

Analogously, we have $4 \pi \gamma^{2} \bar{n}_{\alpha}=2 N \int Y^{\ell}(\alpha-z) \rho_{V}(z) m(\mathrm{~d} z)+\mathrm{O}\left(N \ell^{2} b\right)\left\|\nabla \rho_{V}\right\|_{\infty}$. It follows that

$$
\begin{aligned}
\sum_{\alpha}\left[N n_{\alpha} Q(\alpha)+4 \pi \gamma^{2} n_{\alpha} \bar{n}_{\alpha}\right]= & N \sum_{\alpha} n_{\alpha}\left[Q(\alpha)+2 \int Y^{\ell}(\alpha-z) \mu_{V}(\mathrm{~d} z)\right]+\mathrm{O}\left(N^{2} \ell^{2} b\right)\left\|\nabla \rho_{V}\right\|_{\infty} \\
& +2 N \sum_{\alpha} n_{\alpha} \int Y^{\ell}(\alpha-z)\left[\rho_{V}(z) m(\mathrm{~d} z)-\mu_{V}(\mathrm{~d} z)\right] \\
= & 2 c_{V} N\left(N-n_{B}\right)+\mathrm{O}\left(N^{2} \ell^{2} b\right)\left\|\nabla \rho_{V}\right\|_{\infty} \\
& -2 N^{2} \iint_{D \times B} Y^{\ell}(z-w) \mu_{V}(\mathrm{~d} z) \mu_{V}(\mathrm{~d} w)+\mathrm{O}\left(N^{2} \ell^{3}\left\|\rho_{V}\right\|_{\infty}^{2}\right),
\end{aligned}
$$

where the second equality follows from the Euler-Lagrange equation 4.8 and $\sum_{\alpha} n_{\alpha}=N-n_{B}$, and using that in the computation of $\iint_{D \times B} Y^{\ell}(z-w) \mu_{V}(\mathrm{~d} z) \mu_{V}(\mathrm{~d} w)$, the contribution of the absolutely continuous part of $\mu_{V}$ in $B$ is of order $N^{2} \ell^{2}\left\|\rho_{V}\right\|_{\infty}^{2}$. Using also that $I_{Q, B}-2 c_{V} \mu_{V}(B)=$ $-\iint_{B \times B} Y^{\ell}(z-w) \mu_{V}(\mathrm{~d} z) \mu_{V}(\mathrm{~d} w)$ by 5.13 , in summary, we have proved

$$
\begin{aligned}
\sum_{\alpha} T_{\alpha}\left(n_{\alpha}\right) & +N^{2} I_{Q, B}+2 c_{V} N\left(n_{B}-N \mu_{V}(B)\right)-\sum_{\alpha} h_{\alpha}\left(n_{\alpha}\right)+N \log \ell \\
= & 2 c_{V} N^{2}-N^{2} \iint_{\mathbb{C}^{2}} Y^{\ell}(z-w) \mu_{V}(\mathrm{~d} z) \mu_{V}(\mathrm{~d} w)+\mathrm{O}\left(N^{2} \ell^{2} b\right)\left(\left\|\rho_{V}\right\|_{\infty}+\left\|\nabla \rho_{V}\right\|_{\infty}\right)^{2} .
\end{aligned}
$$

Lemma 4.8 now follows from the Euler-Lagrange equation 4.8, which implies

$$
\begin{aligned}
2 c_{V} & =2 \iint Y^{R}(z-w) \mu_{V}(\mathrm{~d} z) \mu_{V}(\mathrm{~d} w)+\int V(z) \mu_{V}(\mathrm{~d} z) \\
& =\iint Y^{R}(z-w) \mu_{V}(\mathrm{~d} z) \mu_{V}(\mathrm{~d} w)+I_{V}^{R}=\iint Y^{\ell}(z-w) \mu_{V}(\mathrm{~d} z) \mu_{V}(\mathrm{~d} w)+K_{R}^{\ell}+I_{V}^{R} .
\end{aligned}
$$

This completes the proof. 
We need the following bound showing that in the sum over $\mathbf{n}$ the dominant term is $\mathbf{n}=\overline{\mathbf{n}}$. The torus version of this lemma was given in Lemma 3.16 .

Lemma 4.9. Recall the condition (4.10). Suppose that we have a collection of functions $\mathcal{E}_{\alpha}: \mathbb{N} \rightarrow$ $\mathbb{R}$ satisfying $\left|\mathcal{E}_{\alpha}(n)-\mathcal{E}_{\alpha}(m)\right| \leqslant \mathrm{O}\left(|n-m|(n+m)^{\varepsilon}\right)$. Define

$$
\mathcal{E}(\mathbf{n})=\sum_{\alpha \subset D}\left[-2 \pi \gamma^{2}\left(n_{\alpha}-\bar{n}_{\alpha}\right)^{2}+\mathcal{E}_{\alpha}\left(n_{\alpha}\right)\right] .
$$

Assume that $\overline{\mathbf{n}}$ satisfies (4.17) and that $\gamma=\ell / b \geqslant N^{-C}$. Then

$$
\frac{1}{\beta} \log \sum_{\mathbf{n}} \mathrm{e}^{\beta \mathcal{E}(\mathbf{n})+\beta \mathrm{O}\left(n_{B} \log N\right)} \leqslant \mathcal{E}(\overline{\mathbf{n}})+N^{\varepsilon} \mathrm{O}\left(N b^{\prime}+\ell^{-2}\left\|\rho_{V}\right\|_{\infty}\right)
$$

where the sum on $\mathbf{n}$ is under the constraint $N=\sum_{\alpha} n_{\alpha}=n_{B}+\sum_{\alpha \subset D} n_{\alpha}$. Notice that $\mathcal{E}$ contains only contribution from the squares in the bulk.

Proof. By definition,

$$
\begin{aligned}
\frac{1}{\beta} \log \sum_{\mathbf{n}} \mathrm{e}^{\beta \mathcal{E}(\mathbf{n})+\beta \mathrm{O}\left(n_{B} \log N\right)}-\mathcal{E}(\overline{\mathbf{n}})=\frac{1}{\beta} \log \sum_{\mathbf{n}} \mathrm{e}^{\beta(\mathcal{E}(\mathbf{n})-\mathcal{E}(\overline{\mathbf{n}}))+\beta \mathrm{O}\left(n_{B} \log N\right)} \\
\quad=\frac{1}{\beta} \log \sum_{\mathbf{n}} \exp \left[\sum_{\alpha} \beta\left[-2 \pi \gamma^{2}\left(n_{\alpha}-\bar{n}_{\alpha}\right)^{2}+\left(\mathcal{E}_{\alpha}\left(n_{\alpha}\right)-\mathcal{E}_{\alpha}\left(\bar{n}_{\alpha}\right)\right]+\mathrm{O}\left(\beta n_{B} \log N\right)\right] .\right.
\end{aligned}
$$

By the constraint $N=\sum_{\alpha} n_{\alpha}=n_{B}+\sum_{\alpha \subset D} n_{\alpha}$, we can add the factor

$$
\begin{aligned}
\mathbb{1}\left(n_{B}-\bar{n}_{B}=\sum_{\alpha \subset D}\left(\bar{n}_{\alpha}-n_{\alpha}\right)\right) & \leqslant \mathbb{1}\left(\left|n_{B}-\bar{n}_{B}\right| \leqslant \sum_{\alpha \subset D}\left|\bar{n}_{\alpha}-n_{\alpha}\right|\right) \\
& \leqslant \exp \left[-\frac{\beta 2 \pi \gamma^{2}}{2 \#\{\alpha \subset D\}}\left(n_{B}-\bar{n}_{B}\right)^{2}+\frac{\beta 2 \pi \gamma^{2}}{2} \sum_{\alpha \subset D}\left(\bar{n}_{\alpha}-n_{\alpha}\right)^{2}\right],
\end{aligned}
$$

where we used $\mathbb{1}(a \leqslant b) \leqslant \mathrm{e}^{-A a^{2}+A b^{2}}$ for any constant $A>0$ and $\left(\sum_{\alpha \subset D} x_{\alpha}\right)^{2} \leqslant \#\{\alpha \subset$ $D\} \sum_{\alpha \subset D} x_{\alpha}^{2}$ where $\#\{\alpha \subset D\}=\mathrm{O}\left(b^{-2}\right)$ is the number of squares. Thus, at the cost of replacing $2 \pi \gamma^{2}$ by $\pi \gamma^{2}$ in 4.22 , we can add the following factor to the right hand side of (4.22):

$$
\exp \left[-c \beta b^{2} \gamma^{2}\left(n_{B}-\bar{n}_{B}\right)^{2}\right]=\exp \left[-\beta c \ell^{2}\left(n_{B}-\bar{n}_{B}\right)^{2}\right],
$$

where $c$ is a constant of order one. With this preparation, to get an upper bound, we now drop the constraint $\sum_{\alpha} n_{\alpha}=N$ on $\mathbf{n}$, and sum each $n_{\alpha}$ independently. For the bulk squares, we use $\left|\mathcal{E}_{\alpha}(n)-\mathcal{E}_{\alpha}(m)\right| \leq \mathrm{O}\left(|n-m|(n+m)^{\varepsilon}\right)$ and the elementary inequality (3.68), as in the torus case. For the boundary layer $B$, we similarly use

$$
\sum_{n=0}^{\infty} \exp \left[C n \log N-c \ell^{2}(n-m)^{2}\right] \leqslant \mathrm{O}\left(\ell^{-1}\right) \mathrm{e}^{\mathrm{O}\left(m+\ell^{-2}\right)(\log N)^{2}} .
$$

In summary, using $\bar{n}_{\alpha}=\mathrm{O}\left(N b^{2}\right)\left\|\rho_{V}\right\|_{\infty}$ for $\alpha \subset D$ and $\bar{n}_{B}=\mathrm{O}\left(N b^{\prime}\right)$ by (4.17), the left-hand side of 4.22 is of order

$(\log N) \sum_{\alpha \subset D} \gamma^{-2}\left(\bar{n}_{\alpha}+\gamma^{-2}\right)^{2 \varepsilon}\left\|\rho_{V}\right\|_{\infty}+(\log N)^{2}\left(\bar{n}_{B}+\ell^{-2}\right) \leq \mathrm{O}\left(N^{2 \varepsilon} \ell^{-2}\right)\left\|\rho_{V}\right\|_{\infty}+\mathrm{O}\left(N b^{\prime}(\log N)^{2}\right)$.

This completes the proof of the lemma. 
4.5. Existence of free energy of Yukawa gas: proof of Theorem 4.1. The proof of Theorem 4.1 below is analogous to that of Proposition 3.17 .

Proof of Theorem 4.1. We first show that if $1 \geqslant R \geqslant N^{-1 / 2+\sigma}$ there is some $\kappa=\kappa(\sigma)>0$ such that (4.1) holds. Subsequently we will observe that any $\kappa<1 / 24$ is admissable if $R \geqslant 1$. To do this, we apply Propositions 4.5, 4.6 and consider the different error terms.

First, for any choice $N^{-1 / 4} \ll b^{\prime} \ll 1$ the error terms involving $b^{\prime}$ are $N^{\varepsilon} \mathrm{O}\left(n_{B}\right)=N^{\varepsilon} \mathrm{O}\left(N b^{\prime}\right)$ using (4.17). In particular, in the situation of Theorem 1.1. we can choose $b^{\prime}<N^{-\kappa}$ as needed. In the situation of Theorem 4.2 , this error term is $N^{\varepsilon} \mathrm{O}\left(N^{-a}\right)$ as claimed in the remark.

Next we emphasize that, in the upper and lower bounds (Propositions 4.5 and 4.6), the range parameter $\ell$ is not required to be the same, but we always require $\ell \leqslant R$. We denote the value of $\ell$ by $\ell_{+}$for the upper bound and by $\ell_{-}$for the lower bound.

We first consider the case $N^{-1 / 2+\sigma} \leqslant R \leqslant 1$. Take $b=N^{-1 / 2+\sigma / 10}$. For $\ell_{+}=N^{-1 / 2+\sigma / 100}$, the error terms in 4.15 are bounded by $N^{1-\sigma / 1000}$. For $\ell_{-}=N^{-1 / 2+9 \sigma / 100}$, the error terms in 4.16) are also bounded by $N^{1-\sigma / 1000}$ (we used $\bar{n}_{B}=\mathrm{O}\left(N b^{\prime}\right)$ ).

With Lemma 4.8 , for some $\kappa=\kappa(\sigma)>0$ we therefore obtain

$$
\begin{aligned}
& \frac{1}{\beta} \log \int \mathrm{e}^{-\beta H_{V}^{R}(\mathbf{z})} m(\mathrm{~d} \mathbf{z}) \geqslant-N^{2} I_{V}^{R}+N \log R+\frac{1}{\beta} \log \left(\begin{array}{c}
N \\
\overline{\mathbf{n}}
\end{array}\right) \mathrm{e}^{-\beta \sum_{\alpha} h_{\alpha}(\overline{\mathbf{n}})}-\mathrm{O}\left(N^{1-\kappa}\right), \\
& \frac{1}{\beta} \log \int \mathrm{e}^{-\beta H_{V}^{R}(\mathbf{z})} m(\mathrm{~d} \mathbf{z}) \leqslant-N^{2} I_{V}^{R}+N \log R+\frac{1}{\beta} \log \sum_{\mathbf{n}}\left(\begin{array}{c}
N \\
\mathbf{n}
\end{array}\right) \mathrm{e}^{-\beta \sum_{\alpha} h_{\alpha}(\mathbf{n})}+\mathrm{O}\left(N^{1-\kappa}\right) .
\end{aligned}
$$

For the rest of this proof, all summations of $\alpha$ are over $\alpha \subset D$.

By Stirling's formula as in (3.72), and using the definitions of $h$ in (4.18) and of $\mathcal{E}$ in (4.20) with $\mathcal{E}_{\alpha}\left(n_{\alpha}\right)=\left(\frac{1}{2}-\frac{1}{\beta}\right) n_{\alpha} \log \left(n_{\alpha} b^{-2}\right)$, we can rewrite 4.23), 4.24) as

$$
\begin{aligned}
& \frac{1}{\beta} \log \int \mathrm{e}^{-\beta H_{V}^{R}(\mathbf{z})} m(\mathrm{~d} \mathbf{z})+N^{2} I_{V}^{R} \geqslant \mathcal{E}(\overline{\mathbf{n}})+\zeta+N \log R+\frac{1}{\beta} N \log N+\mathrm{O}\left(N^{1-\kappa}\right), \\
& \frac{1}{\beta} \log \int \mathrm{e}^{-\beta H_{V}^{R}(\mathbf{z})} m(\mathrm{~d} \mathbf{z})+N^{2} I_{V}^{R} \leqslant \frac{1}{\beta} \log \sum_{\mathbf{n}} \mathrm{e}^{\beta \mathcal{E}(\mathbf{n})}+\zeta+N \log R+\frac{1}{\beta} N \log N+\mathrm{O}\left(N^{1-\kappa}\right) .
\end{aligned}
$$

By Lemma 4.9, we can replace the sum over $\mathbf{n}$ in 4.26 by the dominant term $\overline{\mathbf{n}}$ with error smaller than $\mathrm{O}\left(N^{1-\kappa}\right)$. By a Riemann sum approximation using that $\rho_{V}$ is $C^{1}$ in $D$,

$$
\begin{aligned}
\mathcal{E}(\overline{\mathbf{n}}) & =\left(\frac{1}{2}-\frac{1}{\beta}\right) \sum_{\alpha} \bar{n}_{\alpha} \log \left(\bar{n}_{\alpha} b^{-2}\right) \\
& =\left(\frac{1}{2}-\frac{1}{\beta}\right) N \int \rho_{V}(z) \log \rho_{V}(z) m(\mathrm{~d} z)+\left(\frac{1}{2}-\frac{1}{\beta}\right) N \log N+\mathrm{O}\left(N\left(b+b^{\prime}\right)\right)\left\|\rho_{V}\right\|_{\infty, 1}
\end{aligned}
$$

This completes the proof of (4.1) when $N^{-1 / 2+\sigma} \leqslant R \leqslant 1$.

To show that if $R \geqslant 1$ then any $\kappa<1 / 24$ is admissible, we consider all error terms in details. In the upper bound (4.15), the error is

$$
\mathrm{O}\left(N^{\varepsilon}\right)\left[N^{2} \ell_{+}^{3} b^{-1}+N^{2} \ell_{+}^{2} b\right]
$$

while, in the lower bound (4.16), it is of order

$$
\mathrm{O}\left(N^{\varepsilon}\right)\left[N^{2} b^{4}+N^{2} \ell_{-}^{2} b+N b+\left(b^{2} \ell_{-}^{-4}+N^{4 / 5} / \ell_{-}^{2 / 5}\right)\right] .
$$


Lemma 4.8 gives analogues of 4.23 and 4.24 with an error term

$$
\mathrm{O}\left(N^{\varepsilon}\right)\left[N^{7 / 8} / b^{1 / 4}+1 / \ell_{-}^{2}+1 / \ell_{+}^{2}\right] \text {. }
$$

Optimizing the parameters yields $b=N^{-1 / 3}, \ell_{+}=N^{-23 / 48}, \ell_{-}=N^{-7 / 18}$. Note that this choice of parameters satisfies the hypothesis $\ell_{-} / b \gg\left(N b^{2}\right)^{-1 / 4}$ and $\ell_{ \pm} \leqslant R$. The common error then becomes $\mathrm{O}\left(N^{23 / 24+\varepsilon}\right)$ for arbitrarily small $\varepsilon>0$. The rest of the proof is unchanged.

4.6. Existence of free energy of Coulomb gas: proof of Theorem 1.1. We now choose $R=N^{2}$ to deduce Theorem 1.1 from Theorem 4.1 .

Proof of Theorem 1.1. The equilibrium measure $\mu_{V}$ of the Coulomb gas in Theorem 1.1 is characterized by the Euler-Lagrange equation

$$
U^{\mu_{V}}+\frac{1}{2} V=c_{V}
$$

in its support $S_{V}$ and inequality in all of $\mathbb{C}$. Define the potential $V_{R}$ via the equation

$$
V_{R}(z)=V(z)+2 \int\left(\log \frac{1}{|z-w|}-Y^{R}(z-w)+Y_{0}+\log R\right) \mu_{V}(\mathrm{~d} w) .
$$

Explicitly, one can check that in $S_{V}$,

$$
U_{R}^{\mu_{V}}+\frac{1}{2} V_{R}=c_{V}^{R}, \quad c_{V}^{R}=c_{V}+Y_{0}+\log R,
$$

holds and with the inequality $\geq c_{V}^{R}$ outside the support of $S_{V}$. Thus $\mu_{V}$ is also the equilibrium measure with respect to the Yukawa interaction and external potential $V_{R}$. Moreover, by (2.2),

$$
\begin{aligned}
I_{V_{R}}^{R} & =\int U_{R}^{\mu_{V}}(z) \mu_{V}(\mathrm{~d} z)+\int V_{R}(z) \mu_{V}(\mathrm{~d} z) \\
& =\int U_{R}^{\mu_{V}}(z) \mu_{V}(\mathrm{~d} z)+\int V(z) \mu_{V}(\mathrm{~d} z)+2 \int\left(U^{\mu_{V}}(z)-U_{R}^{\mu_{V}}(z)+Y_{0}+\log R\right) \\
& =I_{V}^{\mathcal{C}}+\left(Y_{0}+\log R\right)+\mathrm{O}\left(\frac{1}{R}\right) .
\end{aligned}
$$

Thus we have

$$
\frac{1}{\beta} \log \int \mathrm{e}^{-\beta H_{V_{R}}^{Y^{R}}} m(\mathrm{~d} \mathbf{z})=\frac{1}{\beta} \log \int \mathrm{e}^{-\beta H_{V}^{\mathcal{C}}} m(\mathrm{~d} \mathbf{z})-N(N-1)\left(Y_{0}+\log R\right)+\mathrm{O}\left(\frac{N^{2}}{R}\right)
$$

Moreover, (2.2) and an analogous estimate for derivatives of (2.1) imply

$$
\max _{k \leqslant 5}\left\|\nabla^{k}\left(V_{R}-V\right)\right\|_{\infty}=\mathrm{O}\left(\frac{1}{R}\right)
$$

Thus, we may apply Theorem 4.1 with $V$ replaced by $V_{R}$ and with $R=N^{2}$, and Theorem 1.1 then follows with $\zeta_{\beta}^{\mathcal{C}}=\zeta-Y_{0}$. 


\section{Proof of Proposition 4.5: free energy upper bound}

5.1. Upper bound: proof of Proposition 4.5. In this section, the condition $R \geqslant N^{2}$ is imposed. Recall from the proof of Proposition 3.8 that, to each square $u+\alpha$, we associate a map $\Phi_{\alpha}^{u}$ : $u+\alpha \rightarrow \mathbb{T}^{(b)}$ defined by $(3.26)-(3.27)$. Analogously to $(3.28)$, we define a two-body potential

$$
\tilde{Y}_{u}^{\ell}(z, w)=\sum_{\alpha \in \mathbf{S}_{0}} U_{b}^{\ell}\left(\Phi_{\alpha}^{u}(z)-\Phi_{\alpha}^{u}(w)\right) \mathbb{1}_{z \in u+\alpha} \mathbb{1}_{w \in u+\alpha}+Y^{\ell}(z-w) \mathbb{1}_{z \notin D_{u}, w \notin D_{u}}
$$

and $\tilde{Q}_{u}$ by replacing $Q$ in the bulk squares $u+\alpha \subset D_{u}$ by its value at the centers of the squares, and outside $D_{u}$ by adding the equilibrium contribution from the pair interaction with the bulk, i.e.,

$$
\tilde{Q}_{u}(z)=\sum_{\alpha \in \mathbf{S}_{0}} Q(c(u+\alpha)) \mathbb{1}_{z \in u+\alpha}+\left(Q(z)+2 N \int_{D_{u}} Y^{\ell}(z-w) \mu_{V}(\mathrm{~d} w)\right) \mathbb{1}_{z \notin D_{u}}
$$

Denote by $\tilde{H}_{u}^{\ell}$ the corresponding Hamiltonian on $\mathbb{C}^{N}$ :

$$
\tilde{H}_{u}^{\ell}(\mathbf{z})=N \sum_{j} \tilde{Q}_{u}\left(z_{j}\right)+\sum_{i \neq j} \tilde{Y}_{u}^{\ell}\left(z_{i}, z_{j}\right)
$$

The main work towards Proposition 4.5 is contained in the proof of Proposition 5.1 below.

Proposition 5.1. Under the assumptions of Proposition 4.5, there exists $u \in[-b / 2, b / 2)^{2}$ such that (the constant $K_{R}^{\ell}$ is defined in (4.5))

$$
\begin{aligned}
\frac{1}{\beta} \log \int \mathrm{e}^{-\beta H_{V}^{R}(\mathbf{z})} m(\mathrm{~d} \mathbf{z}) \leqslant \frac{1}{\beta} \log \int \mathrm{e}^{-\beta \tilde{H}_{u}^{\ell}(\mathbf{z})} m(\mathrm{~d} \mathbf{z}) & +N \log (R / \ell)+N^{2} K_{R}^{\ell} \\
& +N^{\varepsilon} \mathrm{O}\left(N^{2} \ell^{3} b^{-1}+N^{2} \ell^{2} b\right)\left\|\rho_{V}\right\|_{\infty, 2} .
\end{aligned}
$$

In preparation of the proof, we collect some notation and bounds. We write $\mathbb{E}^{u} f(u)=$ $b^{-2} \int_{[-b / 2, b / 2)^{2}} \mathrm{~d} u f(u)$ for the average over $u$, and analogously to 3.30 , we denote

$$
\bar{Y}(z, w)=\mathbb{E}^{u} \tilde{Y}_{u}^{\ell}(z, w), \quad \bar{Q}(z)=\mathbb{E}^{u} \tilde{Q}_{u}(z) .
$$

The following lemma provides estimates on $\bar{Y}$, extending the analogous Lemma 3.10 for the torus. The estimates are stated in terms of the function $g$ defined in (3.32).

Lemma 5.2. Assume that $\ell \ll b$. Then

(i) Inside the bulk, i.e., for $z, w \in \bigcap D$, we have $\bar{Y}(z, w)=g(z-w)+\mathrm{O}\left(\mathrm{e}^{-c b / \ell}\right)$ and $g(z-$ $w)-Y^{\ell}(z-w)=\mathrm{O}(\ell / b)$.

(ii) Away from the bulk, i.e., for $z, w \notin \bigcup D$, by definition we have $\bar{Y}(z, w)=Y^{\ell}(z-w)$.

(iii) In general, and in particular near the boundary, we have the inequalities

$$
g(z-w)+\mathrm{O}\left(\mathrm{e}^{-c b / \ell}\right) \leqslant \bar{Y}(z, w) \leqslant Y^{\ell}(z-w)+\mathrm{O}\left(\mathrm{e}^{-c b / \ell}\right) \quad \text { if }|z-w|_{\infty} \leqslant b / 2 .
$$

Proof. (i) This case is exactly the same as Lemma 3.10 .

(ii) In this case, since $z, w \notin D_{u}$, by the definition (5.1) we directly have $\bar{Y}(z, w)=Y^{\ell}(z, w)$. 
(iii) By the exponential decay of $Y^{\ell}$, the definition (5.1) and using that $U^{\ell}$ is the periodization of $Y^{\ell}$, we have the bound $\bar{Y}(z, w) \leqslant Y^{\ell}(z-w)+\mathrm{O}\left(\mathrm{e}^{-c b / \ell}\right)$ for $|z-w|_{\infty} \leqslant b / 2$.

For the lower bound on $\bar{Y}$ for $|z-w|_{\infty} \leqslant b / 2$, we notice that $\tilde{Y}_{u}^{\ell}(z, w)=Y^{\ell}(z-w)+\mathrm{O}\left(\mathrm{e}^{-c b / \ell}\right)$ if and only if either $z$ and $w$ belong to the same square $\alpha \subset D_{u}$ or $z, w \notin D_{u}$, and in other cases $\tilde{Y}_{u}^{\ell}(z, w)=0$. The probability of first event, with respect to the $u$-average, is bounded below by that of the event that $z$ and $w$ are both in the same square, irregardless of whether the square is in $D_{u}$ or not. This probability is $(b-x)(b-y) / b^{2}$, and therefore

$$
\tilde{Y}(z, w) \geqslant \frac{(b-x)(b-y)}{b^{2}} Y^{\ell}(z-w)+\mathrm{O}\left(\mathrm{e}^{-c b / \ell}\right)=g(z-w)+\mathrm{O}\left(\mathrm{e}^{-c b / \ell}\right) .
$$

This completes the proof.

Proof of Proposition 5.1. By Jensen's inequality,

$$
\frac{1}{\beta} \log \int \mathrm{e}^{-\beta H_{V}^{R}(\mathbf{z})} m(\mathrm{~d} \mathbf{z}) \leq \frac{1}{\beta} \log \int \mathrm{e}^{-\beta \tilde{H}_{u}^{\ell}(\mathbf{z})} m(\mathrm{~d} \mathbf{z})+\mathbb{E}_{V}^{R}\left(\tilde{H}_{u}^{\ell}-H_{V}^{R}\right),
$$

where we recall that $\mathbb{E}_{V}^{R}$ denotes the expectation of the probability measure with density $\mathrm{e}^{-\beta H_{V}^{R}}$. The last term can be rewritten as

$$
\mathbb{E}_{V}^{R}\left(\tilde{H}_{u}^{\ell}-H_{V}^{R}\right)=\mathbb{E}_{V}^{R}\left(\tilde{H}_{u}^{\ell}-H_{V}^{\ell}\right)+\mathbb{E}_{V}^{R}\left(H_{V}^{\ell}-H_{V}^{R}\right)
$$

Using that $L_{R}^{\ell}$ is positive definite, $\mathbf{L}_{R}^{\ell} \geqslant 0$, and by (4.7), the last term in 5.8 is bounded by

$$
\mathbb{E}_{V}^{R}\left(H_{V}^{\ell}-H_{V}^{R}\right)=-N^{2} \mathbb{E}_{V}^{R} \mathbf{L}_{R}^{\ell}+N \log (R / \ell)+N^{2} K_{R}^{\ell} \leq N \log (R / \ell)+N^{2} K_{R}^{\ell} .
$$

To bound the first term in (5.8) for some $u$, it suffices to bound the average of (5.7) over $u$ in the square $[-b / 2, b / 2]^{2}$. Indeed, by the mean-value theorem for continuous functions, there then exists a choice of $u$ that achieves the bound of the average. By the definition of $\bar{Y}$ and $\bar{Q}$ in (5.5), we have

$\frac{1}{b^{2}} \int_{[-b / 2, b / 2]^{2}} \mathrm{~d} u \mathbb{E}_{V}^{R}\left(\tilde{H}_{u}^{\ell}-H_{V}^{\ell}\right)=\mathbb{E}_{V}^{R}\left[N \sum_{j}\left(\bar{Q}\left(z_{j}\right)-Q\left(z_{j}\right)\right)\right]+\mathbb{E}_{V}^{R}\left[\sum_{i \neq j}\left(\bar{Y}\left(z_{i}, z_{j}\right)-Y^{\ell}\left(z_{i}-z_{j}\right)\right)\right]$.

For the particles in the bulk, the term involving $Q$ is bounded using (4.9). Indeed, the term $2 c_{V}$ in (4.9) cancels and using that $\ell \leqslant b$ and $N^{\varepsilon} \ell^{4} \leqslant \ell^{2} b$ the difference of the other two terms in $(4.9)$ is estimated by

$$
N \sum_{j}\left(Q\left(z_{j}\right)-\bar{Q}\left(z_{j}\right)\right) \mathbb{1}_{z_{j} \in D}=\mathrm{O}\left(N^{2} \ell^{2} b\right)\left(\left\|\nabla \rho_{V}\right\|_{\infty}+\left\|\nabla^{2} \rho_{V}\right\|_{\infty}\right)
$$

For the particles outside the bulk, the difference of $Q$ and $\tilde{Q}$ is by the definition 5.2 equal to

$$
2 N \int_{D} Y^{\ell}\left(z_{j}-w\right) \mu_{V}(\mathrm{~d} w)=\mathrm{O}\left(N \ell^{2}\right)\left\|\rho_{V}\right\|_{\infty}
$$

By the decay of the Yukawa potential, only particles $z_{j}$ within distance $N^{\varepsilon} \ell$ to $D$ give a nonnegligible contribution to this term. By the local density estimate, Theorem 2.3 , there are $\mathrm{O}\left(N^{1+\varepsilon} \ell\right)$ such particles, so that the sum of the last expression over the particles $z_{j}$ in the boundary region is bounded by $N^{2+\varepsilon} \ell^{3} \leqslant N^{2} \ell^{2} b$. 
Similarly, dividing the sum over $i \neq j$ for the pair interaction in 5.9 into bulk and boundary contribution, Lemma 5.3 below implies

$$
\mathbb{E}_{V}^{R} \sum_{i \neq j}\left[\bar{Y}\left(z_{i}, z_{j}\right)-Y^{\ell}\left(z_{i}-z_{j}\right)\right]=N^{\varepsilon} \mathrm{O}\left(N^{2} \ell^{3} b^{-1}\right) .
$$

Here we used that the contribution for the above sum where both $z_{i}$ and $z_{j}$ are outside $\bigcup D$ vanishes since then $\bar{Y}\left(z_{i}, z_{j}\right)=Y^{\ell}\left(z_{i}-z_{j}\right)$. Moreover, for the contributions where at least one of the particles is in the bulk, we may assume with negligible error that the other particle is at most distance $N^{\varepsilon} \ell$ from it and thus also far from the boundary so that the local density estimate is applicable. This completes the proof.

The following lemma is analogous to Lemma 3.11 for the torus.

Lemma 5.3. For any $u$,

$$
\begin{aligned}
\mathbb{E}_{V}^{R} \sum_{i, j} \mathbb{1}_{z_{i}, z_{j} \in \cap D}\left[g\left(z_{i}-z_{j}\right)-Y^{\ell}\left(z_{i}-z_{j}\right)\right] & =\mathrm{O}\left(N^{\varepsilon} N^{2} \ell^{3} b^{-1}\right), \\
\mathbb{E}_{V}^{R} \sum_{i, j} \mathbb{1}_{z_{i} \in B, z_{j} \in \cup D}\left[\tilde{Y}\left(z_{i}, z_{j}\right)-Y^{\ell}\left(z_{i}-z_{j}\right)\right] & =\mathrm{O}\left(N^{\varepsilon} N^{2} \ell^{3}\right) .
\end{aligned}
$$

Proof. We use the local density for the Yukawa gas, Theorem 2.3, stating that balls of radius $r \gg N^{-1 / 2}$ contain $\mathrm{O}\left(N r^{2}\right)$ particles with high probability (provided that the distance to the boundary is at least $\left.b^{\prime} \gg N^{-1 / 4}\right)$. In addition, we use that for $\left|z_{i}-z_{j}\right| \geqslant \ell N^{\varepsilon}$ we have $Y^{\ell}\left(z_{i}-z_{j}\right) \leqslant \mathrm{e}^{-c N^{\varepsilon}}$ so that contributions to the corresponding contributions to the double sums in the statement contribute lower order errors. As a consequence, exactly as in the proof of 3.37 ,

$$
\mathbb{E}_{V}^{R} \sum_{i, j}\left[g\left(z_{i}-z_{j}\right)-Y^{\ell}\left(z_{i}-z_{j}\right)\right]=\mathrm{O}\left(N^{\varepsilon} N\left(N \ell^{2}\right)(\ell / b)\right)
$$

since each of the at most $N$ particles $z_{i}$ interacts with $\mathrm{O}\left(N^{\varepsilon} N \ell^{2}\right)$. particles $z_{j}$, and the difference $g-Y^{\ell}$ is of order $\ell / b$ by Lemma 5.2 (i). This proves (5.11).

The estimate for the boundary layer $(5.12)$ is analogous. Indeed, by definition, the boundary layer has distance at least $b^{\prime}$ to the boundary of the support of the equilibrium measure, so that the local density estimate can still be applied. Then we similarly have

$$
\mathbb{E}_{V}^{R} \sum_{i, j} \mathbb{1}_{z_{i} \in B, z_{j} \in \bigcup D}\left[\tilde{Y}\left(z_{i}, z_{j}\right)-Y^{\ell}\left(z_{i}-z_{j}\right)\right]=\mathrm{O}\left((N \ell)\left(N \ell^{2}\right)\right) .
$$

To see that this inequality holds, we note that, up to exponentially small errors, the only pairs we need to consider are that one particle is in the boundary and the other one is in the bulk with the distance of these two particles of order $\ell$. Since, by the local density estimate, with high probability the total number of particle near boundary corridor of width $\ell$ is $N \ell$ and each particle interacts with $N \ell^{2}$ particles, the left side of the last inequality is of order $N \ell N \ell^{2}$.

To bound the boundary contribution, we will need the following estimate. For $z \in S_{V} \backslash D$, recall from (5.2) and the Euler-Lagrange equation (4.8) that

$$
\tilde{Q}(z)=Q(z)+2 \int_{D} Y^{\ell}(z-w) \mu_{V}(\mathrm{~d} w)=2 c_{V}-2 \int_{B} Y^{\ell}(z-w) \mu_{V}(\mathrm{~d} w),
$$


and define the constant

$$
\begin{aligned}
I_{Q, B} & =\int_{B} \tilde{Q}(z) \mu_{V}(\mathrm{~d} z)+\iint_{B^{2}} Y^{\ell}(z-w) \mu_{V}(\mathrm{~d} z) \mu_{V}(\mathrm{~d} w) \\
& =2 c_{V} \mu_{V}(B)-\iint_{B^{2}} Y^{\ell}(z-w) \mu_{V}(\mathrm{~d} z) \mu_{V}(\mathrm{~d} w) .
\end{aligned}
$$

Proposition 5.4. For any $u$,

$$
\begin{aligned}
\frac{1}{\beta} \log \int_{\left(\mathbb{C} \backslash D_{u}\right)^{n} B} \mathrm{e}^{-\beta N \sum_{j} \tilde{Q}\left(z_{j}\right)-\beta \sum_{j \neq k} Y^{\ell}\left(z_{j}-z_{k}\right)} m(\mathrm{~d} \mathbf{z}) & \\
& \leqslant-N^{2} I_{Q, B}-2 c_{V} N\left(n_{B}-N \mu_{V}\left(B_{u}\right)\right)+\mathrm{O}\left(n_{B} \log N\right) .
\end{aligned}
$$

Proof. We fix $u$ and abbreviate $D=D_{u}$ and $B=B_{u}$ throughout the proof. Let

$$
E\left(n_{B}\right)=\inf _{\int \omega=n_{B}}\left[N \int_{\mathbb{C} \backslash D} \tilde{Q}(z) \omega(\mathrm{d} z)+\iint_{(\mathbb{C} \backslash D)^{2}} Y^{\ell}(z-w) \omega(\mathrm{d} z) \omega(\mathrm{d} w)\right],
$$

where $\omega$ is a positive measure of total mass $n_{B}$ supported on $\mathbb{C} \backslash D$. Using the standard technique to replace point particle by a smooth distribution of radius $1 / N$, the left-hand side of (5.14) is bounded above by

$$
-E\left(n_{B}\right)+\mathrm{O}\left(n_{B} \log N\right) .
$$

(A more sophisticated form of this method will be presented in the proof of Proposition B.8 where the regularity of the equilibrium measure was used only in the proof of the lower bound of the partition function.)

It thus suffices to show that

$$
E\left(n_{B}\right)-N^{2} I_{Q, B} \geqslant 2 c_{V} N\left(n_{B}-N \mu_{V}(B)\right) .
$$

To do so, with $\tilde{\omega}=\omega-N \mu_{V}$ inside the infimum, we write

$$
\begin{aligned}
& E\left(n_{B}\right)-N^{2} I_{Q, B} \\
& =\inf _{\int \omega=n_{B}}\left[N \int_{D^{c}} \tilde{Q}(z) \omega(\mathrm{d} z)+\iint_{\left(D^{c}\right)^{2}} Y^{\ell}(z-w) \omega(\mathrm{d} z) \omega(\mathrm{d} w)\right] \\
& \quad-N^{2} \int_{D^{c}} \tilde{Q}(z) \mu_{V}(\mathrm{~d} z)-N^{2} \iint_{\left(D^{c}\right)^{2}} Y^{\ell}(z-w) \mu_{V}(\mathrm{~d} z) \mu_{V}(\mathrm{~d} w) \\
& =\inf _{\int \omega=n_{B}}\left[N \int_{D^{c}} \tilde{\omega}(\mathrm{d} z)\left[\tilde{Q}(z)+2 \int_{D^{c}} Y^{\ell}(z-w) \mu_{V}(\mathrm{~d} w)\right]+\int_{D^{c}} Y^{\ell}(z-w) \tilde{\omega}(\mathrm{d} z) \tilde{\omega}(\mathrm{d} w)\right] .
\end{aligned}
$$

The last term on the right-hand side is nonnegative, and can therefore be dropped. By definition of $\tilde{Q}$ and the Euler-Lagrange equation 4.8 , also

$$
\tilde{Q}(z)+2 \int_{D^{c}} Y^{\ell}(z-w) \mu_{V}(\mathrm{~d} w)=Q(z)+2 \int Y^{\ell}(z-w) \mu_{V}(\mathrm{~d} w) \geqslant 2 c_{V} .
$$

Since the same relation holds with equality on the support of $\mu_{V}$, therefore

$$
E\left(n_{B}\right)-N^{2} I_{Q, B} \geqslant 2 c_{V} N \int_{D^{c}} \tilde{\omega}(\mathrm{d} z)=2 c_{V} N\left(n_{B}-N \mu_{V}(B)\right) .
$$

This completes the proof. 
Proof of Proposition 4.5. Summing over the possible particle profiles, we have

$$
\int \mathrm{e}^{-\beta \tilde{H}_{u}^{\ell}(\mathbf{z})} m(\mathrm{~d} \mathbf{z})=\sum_{\mathbf{n}}\left(\begin{array}{l}
N \\
\mathbf{n}
\end{array}\right) \int \mathrm{e}^{-\beta \tilde{H}_{u}^{\ell}(\mathbf{z})} \mathbb{1}_{\mathbf{n}(\mathbf{z})=\mathbf{n}} m(\mathrm{~d} \mathbf{z})
$$

where $\mathbf{n}(\mathbf{z})$ is the particle profile of the configuration $\mathbf{z} \in \mathbb{C}^{N}$. By definition of $\tilde{H}$, for any $u$, the integral on the right-hand side factorizes as

$$
\left(\prod_{\alpha \subset D} \int_{\alpha^{n} n^{n}} \mathrm{e}^{-\beta \hat{H}_{\alpha}(\mathbf{z})} m(\mathrm{~d} z)\right) \times\left(\int_{(\mathbb{C} \backslash D)^{n_{B}}} \mathrm{e}^{-\beta N \sum_{j} \tilde{Q}\left(z_{j}\right)-\beta \sum_{j \neq k} Y^{\ell}\left(z_{j}-z_{k}\right)} m(\mathrm{~d} \mathbf{z})\right) .
$$

The claim now follows from Propositions 5.1 and 5.4 .

5.2. Summary. We summarize some of the key facts used in the proof of the upper bound of the partition function of the Yukawa gas:

(i) The local densities are bounded at the scale $\ell$ of the interaction.

(ii) The solution of the ground state is regular in terms of derivatives of $\rho_{V}$; this is reflected in the estimate (5.4).

(iii) We used the independent particle approximation for particles within distance $b^{\prime}$ to the boundary of the support of the equilibrium measure. In order to control the error due to the interactions between boundary particles and bulk particles, we used that the local density at the scale $\ell$ for particles at a distance of order $b^{\prime}$ to the boundary is bounded.

\section{Decoupling estimate}

The proof for the lower bound on the partition function, Proposition 4.6, will be presented in Section 7. This proof is based as on a trial state similar to the one used in Section 3.6 for the torus case. Notice that the Yukawa potential has range $R$ in the current setting instead of $\ell$ in the torus case. Since our grid size $b$ satisfies $\ell \ll b \ll R$, many error terms which are negligible in the torus case need now to be estimated carefully. In particular, the embedding map $\Psi$ has to be chosen differently from the simple average used in (3.42)-(3.43). In preparation of Section 7 . we construct this choice in Proposition 6.1 below. We call it a decoupling estimate because it allows us to pass from the original Yukawa gas to the quasi-free Yukawa gas in which cubes are decoupled. By rescaling, we state the estimates for the Yukawa gas on the unit torus.

More precisely, the next proposition asserts the existence of a random choice of maps

$$
\Psi: \mathbb{T} \rightarrow[-1 / 2,1 / 2)^{2} \subset \mathbb{R}^{2} \quad \text { with Jacobian }|\mathrm{d} \Psi|=1,
$$

such that the estimates stated in the proposition hold. Here $\mathbb{T}$ is the unit torus. The expectation corresponding to the randomness defining the maps $\Psi$ is denoted by $\mathbb{E}^{\Psi}$ (and is independent of everything else). In the statement of the estimate in the following proposition, $U^{\gamma}$ is the Yukawa potential on the unit torus (2.4), $Y^{\gamma}$ is the Yukawa potential on the plane (2.1), but $\mathbb{E}^{\gamma}$ denotes the expectation of the Yukawa gas with $N$ particles and range $\gamma$ on the unit torus. As usually, we also denote $\hat{\mu}$ the empirical measure and $\tilde{\mu}=\hat{\mu}-m$ where $m$ is the uniform probability measure on $\mathbb{T}$. In the statement below and this section, it is understood that all double integrals are evaluated on $\{z \neq w\}$. 
Proposition 6.1. Assume that $N^{-1 / 4} \ll \gamma \ll 1$ and $\gamma \leqslant R$. Let $\mathbb{E}^{\gamma}$ denote the expectation of the $N$-particle Yukawa gas of range $\gamma$ on the unit torus $\mathbb{T}$. There is a random choice of $\Psi: \mathbb{T} \rightarrow[-1 / 2,1 / 2)^{2}$ with $|\mathrm{d} \Psi|=1$ such that

$$
\begin{array}{r}
N^{2} \mathbb{E}^{\Psi} \mathbb{E}^{\gamma} \iint_{\mathbb{T} \times \mathbb{T}}\left(U^{\gamma}(v-w)-Y^{\gamma}(\Psi(v)-\Psi(w))\right) \tilde{\mu}(\mathrm{d} v) \tilde{\mu}(\mathrm{d} w)=N^{\varepsilon} \mathrm{O}\left(N^{4 / 5} / \gamma^{2 / 5}+\gamma^{-4}\right), \\
N^{2} \mathbb{E}^{\Psi} \mathbb{E}^{\gamma} \iint_{\mathbb{T} \times \mathbb{T}}\left(Y^{R}(\Psi(v)-\Psi(w))-Y^{\gamma}(\Psi(v)-\Psi(w))\right) \tilde{\mu}(\mathrm{d} v) \tilde{\mu}(\mathrm{d} w)=N^{\varepsilon} \mathrm{O}\left(N^{4 / 5} / \gamma^{2 / 5}+\gamma^{-4}\right) .
\end{array}
$$

The remainder of this section is devoted to the proof of this proposition. The main reason to introduce randomness into $\Psi$ is to resolve the issue that the torus distance and Euclidean distance are incompatible. The range of the Yukawa interaction $\ell$, appearing in the quasi-free gas, is small. On the other hand, we wish to use it to approximate the Coulomb energy which corresponds to $Y^{R}$ with $R \gg 1$. The Coulomb interaction will be pushed back to the torus; this creates discontinuities since the torus is periodic. The naive embedding of the square onto the torus used in Section 3.4 is discontinuous along a horizontal and a vertical line. This discontinuity can be averaged out using the translational invariance of the torus, but the resulting interaction on the torus is still not smooth enough to apply the rigidity estimate. Therefore we now choose $\Psi$ to involve a more sophisticated average than the simple mean over the discontinuity lines so as to have a smooth interaction after pushing back the Coulomb interaction to the torus.

In Section 7, we will apply this estimate with the unit torus $\mathbb{T}$ rescaled to the torus $\mathbb{T}^{(b)}$ of side length $b$. For later reference, we state the rescaled version below.

Corollary 6.2. Let $\ell \ll b \ll 1, \ell \leqslant R$, and assume that $\gamma:=\ell / b$ satisfies $n^{-1 / 4} \ll \gamma \ll 1$. Let $\mathbb{E}_{b}^{\ell}$ denote the expectation of the n-particle Yukawa gas of range $\ell$ on the torus $\mathbb{T}^{(b)}$. Then there is a random choice of $\Psi=\Psi^{(b)}: \mathbb{T}^{(b)} \rightarrow[-b / 2, b / 2)^{2}$ with $|\mathrm{d} \Psi|=1$ such that

$$
\begin{array}{r}
n^{2} \mathbb{E}^{\Psi} \mathbb{E}_{b}^{\ell} \iint_{\mathbb{T}^{(b)} \times \mathbb{T}^{(b)}}\left(U_{b}^{\ell}(v-w)-Y^{\ell}(\Psi(v)-\Psi(w))\right) \tilde{\mu}(\mathrm{d} v) \tilde{\mu}(\mathrm{d} w)=n^{\varepsilon} \mathrm{O}\left(n^{4 / 5} / \gamma^{2 / 5}+\gamma^{-4}\right), \\
n^{2} \mathbb{E}^{\Psi} \mathbb{E}_{b}^{\ell} \iint_{\mathbb{T}^{(b)} \times \mathbb{T}^{(b)}}\left(Y^{R}(\Psi(v)-\Psi(w))-Y^{\ell}(\Psi(v)-\Psi(w))\right) \tilde{\mu}(\mathrm{d} v) \tilde{\mu}(\mathrm{d} w)=n^{\varepsilon} \mathrm{O}\left(n^{4 / 5} / \gamma^{2 / 5}+\gamma^{-4}\right) .
\end{array}
$$

Proof. The corollary is immediate from Proposition 6.1 by rescaling.

6.1. Choice of the maps $\Psi_{\alpha}$. To define the maps $\Psi$, we define $[u]$ through

$$
-\frac{1}{2} \leqslant[u]<\frac{1}{2}, u-[u] \in \mathbb{Z} \quad \text { for } u \in \mathbb{R}, \quad[z]=\left(\left[z_{1}\right],\left[z_{2}\right]\right) \in \mathbb{T} \quad \text { for } z \in \mathbb{C} \cong \mathbb{R}^{2} .
$$

Then we define maps $\Phi_{1}, \Phi_{2}: \mathbb{T} \rightarrow \mathbb{T}$ by

$$
\Phi_{1}(z)=\left(\left[z_{1}+m_{1} s\left(z_{2}\right)\right], z_{2}\right), \quad \Phi_{2}(z)=\left(z_{1},\left[z_{2}+m_{2} s\left(z_{1}\right)\right]\right),
$$

where we will choose $s(z)=\sin (2 \pi x)$ (or any smooth periodic function with similar oscillation). Let $\Phi=\Phi_{1} \circ \Phi_{2}$. We choose $m_{1}, m_{2}$ as independent random variables with the distribution of 
$t X$ with $X$ a random variable with smooth and compactly supported density, $\mathbb{E}(X)=0$, and $N^{-1 / 2} \ll t \ll 1$ is some mesoscopic scale. Eventually, we will choose

$$
t=N^{-1 / 4} \text {. }
$$

Finally, let $\Psi_{z}=\left[\Phi(z)+\left(a_{1}, a_{2}\right)\right]$, where $\left(a_{1}, a_{2}\right)$ is a random shift, with $a_{1}$ and $a_{2}$ independent and uniform on $[-1 / 2,1 / 2)$. Note that $\Phi$ and $\Psi$ are smooth function on the torus and they preserve volumes:

$$
|\mathrm{d} \Phi|=|\mathrm{d} \Psi|=1 .
$$

6.2. From euclidean to periodic interaction. All terms we need to bound can be written as in the left-hand side of 2.25, so Proposition 2.5 will be our main tool. However, these terms involve interactions for the Euclidean distance on the square while Proposition 6.1 applies to the unit torus. We therefore first need the next Lemma 6.4 to turn the Euclidean interaction into a periodic one; subsequently, we decompose the resulting singularities carefully. For the lemma, we first need the following definition of an average of interaction over translations.

Definition 6.3. For any $G: \mathbb{T}^{2} \rightarrow \mathbb{R}$ and $h \in \mathbb{C}$, we define

$$
\mathcal{T}_{G}(h)=\int_{\mathbb{T}} G(z,[z+h]) m(\mathrm{~d} z),
$$

where $m$ is the Lebesgue measure on $\mathbb{T}$ and we used the notation (6.6).

If $G(z, w)=g(|z-w|)$ is a function of the Euclidean distance, $\widetilde{\mathcal{T}}_{G}$ will also be denoted by $\mathcal{T}_{g}$ (and is obviously equal to $\left.\mathcal{T}_{g}(h)=\int_{\mathbb{T}} g(|[z+h]-z|) m(\mathrm{~d} z)\right)$.

We remark that in the above definition and below, $z-w$ for $(z, w) \in \mathbb{T} \times \mathbb{T}$ is defined as the difference of two elements in $\mathbb{C}^{2}$ through the identification of $\mathbb{T}=[-1 / 2,1 / 2)^{2}$.

Lemma 6.4. Consider a Yukawa gas on the unit torus $\mathbb{T}, G: \mathbb{T}^{2} \rightarrow \mathbb{R}$, and assume all integrands below are integrable. The following holds:

$$
\mathbb{E} \iint_{z \neq w} G(z, w) \hat{\mu}(\mathrm{d} z) \hat{\mu}(\mathrm{d} w)=\mathbb{E} \iint_{z \neq w} \mathcal{T}_{G}([z-w]) \hat{\mu}(\mathrm{d} z) \hat{\mu}(\mathrm{d} w) .
$$

Moreover, if $G(z, w)=g(|z-w|)$ is a function of the Euclidean distance, for any $h=\left(h_{1}, h_{2}\right) \in \mathbb{T}$ we have

$$
\begin{gathered}
\mathcal{T}_{g}(h)=\left(1-\left|h_{1}\right|\right)\left(1-\left|h_{2}\right|\right) g_{1}(h)+\left|h_{1}\right|\left(1-\left|h_{2}\right|\right) g_{2}(h) \\
+\left|h_{2}\right|\left(1-\left|h_{1}\right|\right) g_{3}(h)+\left|h_{1}\right|\left|h_{2}\right| g_{4}(h),
\end{gathered}
$$

where

$$
\begin{array}{ll}
g_{1}(h):=g\left(\sqrt{\left|h_{1}\right|^{2}+\left|h_{2}\right|^{2}}\right), & g_{2}(h):=g\left(\sqrt{\left(1-\left|h_{1}\right|\right)^{2}+\left|h_{2}\right|^{2}}\right), \\
g_{3}(h):=g\left(\sqrt{\left|h_{1}\right|^{2}+\left(1-\left|h_{2}\right|\right)^{2}}\right), & g_{4}(h):=g\left(\sqrt{\left(1-\left|h_{1}\right|\right)^{2}+\left(1-\left|h_{2}\right|\right)^{2}}\right) .
\end{array}
$$

Remark 6.5. The above calculation is stated for $h \in \mathbb{T}$, and it shows that $\mathcal{T}_{G}=\mathcal{T}_{g}$ is not smooth for $h_{1}=0$ or $h_{2}=0$. This non-smoothness prevents us from using the rigidity estimate (2.23) and is the main source of difficulty we will address in this section. In addition to the non-smoothness for $h_{1}=0$ or $h_{2}=0$, one may wonder if $\mathcal{T}_{G}$ has additional singularities (i.e., non-smoothness) at $h_{1}= \pm 1 / 2$ or $h_{2}= \pm 1 / 2$, as a function on the torus. It has not, as shown by the following argument. Assume $-1 / 2 \leqslant h_{2}<1 / 2$ is fixed. The right-hand side of (6.11) admits an obvious smooth extension to $h_{1} \in(0,1)$, called $\tilde{\mathcal{T}}_{G}$. One readily sees that for such $h_{1} \in(0,1)$, we have $\tilde{\mathcal{T}}_{G}\left(h_{1}, h_{2}\right)=\tilde{\mathcal{T}}_{G}\left(1-h_{1}, h_{2}\right): \tilde{\mathcal{T}}_{G}$ is smooth and symmetric with respect to $h_{1}=1 / 2$, so all its odd derivatives vanish there, meaning $\mathcal{T}_{G}$ is smooth at $h_{1}= \pm 1 / 2$. The same reasoning applies on $h_{2}= \pm 1 / 2$. 
Proof. Recall that $\rho_{2}$ is the two point correlation function for the Yukawa gas on $\mathbb{T}$. By translation invariance of the distribution of the Yukawa gas, we have

$$
\begin{aligned}
& \mathbb{E} \iint_{z \neq w} G(z, w) \hat{\mu}(\mathrm{d} z) \hat{\mu}(\mathrm{d} w)=\iint_{z \neq w} G(z, w) \rho_{2}([z-w]) m(\mathrm{~d} z) m(\mathrm{~d} w) \\
& =\iint G(z,[z+h]) \rho_{2}(h) m(\mathrm{~d} z) m(\mathrm{~d} h)=\int \rho_{2}(h)\left(\int G(z,[z+h]) m(\mathrm{~d} z)\right) m(\mathrm{~d} h) \\
& =\int \rho_{2}(h) \mathcal{T}_{G}(h) m(\mathrm{~d} h)=\iint \rho_{2}(h) \mathcal{T}_{G}(h) m(\mathrm{~d} h) m(\mathrm{~d} \tilde{z})=\mathbb{E} \iint_{z \neq w} \mathcal{T}_{G}([\tilde{z}-\tilde{w}]) \hat{\mu}(\mathrm{d} \tilde{z}) \hat{\mu}(\mathrm{d} \tilde{w}) .
\end{aligned}
$$

In the case $G(z, w)=g(|z-w|)$, the assertion follows from a direct calculation of $\mathcal{T}_{G}(h)=$ $\int_{\mathbb{T}} g(|[z+h]-z|) m(\mathrm{~d} z)$.

Denote by $\mathbb{E}_{(a, b)}$ integration with respect to the shift $(a, b)$ of $\Psi$, and write

$$
\Delta_{z}^{w}=\left[\Phi_{z}-\Phi_{w}\right]
$$

Then the functional $\mathcal{T}$ from Definition 6.3 naturally appears in the following calculation:

$$
\mathbb{E}_{(a, b)}\left(G\left(\Psi_{z}, \Psi_{w}\right)\right)=\int_{\mathbb{T}} G\left(\left[\Psi_{z}+\tilde{z}\right],\left[\Psi_{w}+\tilde{z}\right]\right) m(\mathrm{~d} \tilde{z})=\int_{\mathbb{T}} G\left(\left[\tilde{z}+\Delta_{z}^{w}\right], \tilde{z}\right) m(\mathrm{~d} \tilde{z})=\mathcal{T}_{G}\left(\Delta_{z}^{w}\right) .
$$

In particular,

$$
\mathbb{E}_{(a, b)}\left(g\left(\left|\Psi_{z}-\Psi_{w}\right|\right)\right)=\mathcal{T}_{g}\left(\Delta_{z}^{w}\right) .
$$

This will be useful in the following proof of Proposition 6.1.

6.3. Proof of estimate 6.2). First note that, by $(2.4)$ we have $U^{\gamma}(z-w)=Y^{\gamma}([z-w])+\mathrm{O}\left(\mathrm{e}^{-N^{c}}\right)$, so that it will be sufficient to prove both of the following estimates:

$$
\begin{aligned}
\mathbb{E}^{\Psi} \mathbb{E}^{\gamma} N^{2} \iint\left(Y^{\gamma}([z-w])-Y^{\gamma}\left(\left[\Psi_{z}-\Psi_{w}\right]\right)\right) \tilde{\mu}(\mathrm{d} z) \tilde{\mu}(\mathrm{d} w) & =\mathrm{O}\left(N^{1+\varepsilon} t\right), \\
\mathbb{E}^{\Psi} \mathbb{E}^{\gamma} N^{2} \iint\left(Y^{\gamma}\left(\left[\Psi_{z}-\Psi_{w}\right]\right)-Y^{\gamma}\left(\Psi_{z}-\Psi_{w}\right)\right) \tilde{\mu}(\mathrm{d} z) \tilde{\mu}(\mathrm{d} w) & =\mathrm{O}\left(N^{\varepsilon}\right)\left(\frac{1}{\gamma^{4}}+\frac{\sqrt{N}}{t}\right) .
\end{aligned}
$$

From (6.8) and the hypothesis $\gamma \ll 1$, the sum of both error terms above is dominated by the right hand side of $(6.3)$.

For the proof of 6.15 , let $N^{-1 / 2} \ll r \ll \gamma$ be some intermediate scale. Let $\chi: \mathbb{R}_{+} \rightarrow[0,1]$ be a smooth function such that $\chi(z)=1$ on $[0,1], \chi(z)=0$ on $[2, \infty)$ and define $q=Y^{\gamma}$, $\widetilde{q}(z)=q(z) \chi(|z| / r)$. The proof of (6.15) will consist of the following two estimates (note that $\left.\left[\Psi_{z}-\Psi_{w}\right]=\left[\Phi_{z}-\Phi_{w}\right]\right)$ :

$$
\begin{array}{r}
\mathbb{E}^{\Phi} \mathbb{E}^{\gamma} N^{2} \iint\left(\widetilde{q}\left(\left[\Phi_{z}-\Phi_{w}\right]\right)-\widetilde{q}([z-w])\right) \widetilde{\mu}(\mathrm{d} z) \widetilde{\mu}(\mathrm{d} w)=N^{\varepsilon} \mathrm{O}\left(N^{2} t^{2} r^{2}\right), \\
\mathbb{E}^{\Phi} \mathbb{E}^{\gamma} N^{2} \iint\left((q-\widetilde{q})\left(\left[\Phi_{z}-\Phi_{w}\right]\right)-(q-\widetilde{q})([z-w])\right) \widetilde{\mu}(\mathrm{d} z) \widetilde{\mu}(\mathrm{d} w)=N^{\varepsilon} \mathrm{O}\left(\frac{1}{r^{2}}\right) .
\end{array}
$$

Optimization over $r$ shows that 6.15 holds (note that the optimum $r^{*}=(N t)^{-1 / 2}$ is smaller than $\gamma$ because $t=N^{-\frac{1}{4}}<\gamma$.

For (6.17), we proceeds by Taylor expansion around $|z-w+a|<N^{\varepsilon} r$, where $a= \pm 1, \pm \mathrm{i}$. We treat the case $a=0$, the other ones being identical. As $\tilde{q}$ is supported on $|x|<N^{\varepsilon} r$, for all 
$z, w$ contributing to 6.17 we have $[z-w]=z-w$ and $\left[\Phi_{z}-\Phi_{w}\right]=\Phi_{z}-\Phi_{w}$. For such $z, w$, from the definition $\Phi=\Phi_{1} \circ \Phi_{2}$ with 6.7 , we have

$$
\left[\Phi_{w}-\Phi_{z}\right]=[w-z]+\left(\begin{array}{l}
m_{1}\left(s\left(w_{2}+m_{2} s\left(w_{1}\right)\right)-s\left(z_{2}+m_{2} s\left(z_{1}\right)\right)\right) \\
m_{2}\left(s\left(w_{1}\right)-s\left(z_{1}\right)\right)
\end{array}\right) .
$$

Expanding 6.19,

$$
\begin{aligned}
& \frac{\left(\left[\Phi_{w}-\Phi_{z}\right]\right)_{2}-([w-z])_{2}}{m_{2}}=s^{\prime}\left(w_{1}\right)\left(w_{1}-z_{1}\right)+\mathrm{O}\left(|w-z|^{2}\right), \\
& \frac{\left(\left[\Phi_{w}-\Phi_{z}\right]\right)_{1}-([w-z])_{1}}{m_{1}}=s^{\prime}\left(w_{2}\right)\left(w_{2}-z_{2}\right)+\mathrm{O}\left(|w-z|^{2}\right)+m_{2} \mathrm{O}(|w-z|)+m_{2}^{2} \mathrm{O}\left(|w-z|^{2}\right),
\end{aligned}
$$

where, here and in the following, the O error terms are non-random, namely, they do not depend on $m_{1}, m_{2}$. Denoting

$$
\Delta=\left(\begin{array}{l}
m_{1}\left(s^{\prime}\left(w_{2}\right)\left(w_{2}-z_{2}\right)+\mathrm{O}\left(|w-z|^{2}\right)+m_{2} \mathrm{O}(|w-z|)+m_{2}^{2} \mathrm{O}\left(|w-z|^{2}\right)\right) \\
m_{2}\left(s^{\prime}\left(w_{1}\right)\left(w_{1}-z_{1}\right)+\mathrm{O}\left(|z-w|^{2}\right)\right)
\end{array}\right)
$$

we have

$$
\widetilde{q}\left(\left[\Phi_{z}-\Phi_{w}\right]\right)-\widetilde{q}([z-w])=\nabla \widetilde{q}([z-w]) \cdot \Delta+\mathrm{O}\left(\sup _{[|z-w| / 2,2|z-w|]}\left|\nabla^{2} \widetilde{q}\right|\right)|\Delta|^{2} .
$$

As $m_{1}, m_{2}$ are centered (under the random choice of $\Phi$ ), the linear terms vanish under expectation. This gives, for any fixed small $\varepsilon>0$,

$$
\mathbb{E}^{\Phi}\left(\widetilde{q}\left(\left[\Phi_{z}-\Phi_{w}\right]\right)-\widetilde{q}([z-w])\right)=\mathbb{E}\left(m^{2}\right)\left|\left(\nabla^{2} \widetilde{q}\right)(z-w)\right| \mathrm{O}\left(|z-w|^{2}\right)=\mathrm{O}\left(N^{2} t^{2}\right) \mathbb{1}_{|z-w| \leqslant N^{\varepsilon} r}
$$

We have therefore proved that

$\mathbb{E}^{\Phi} \mathbb{E}^{\gamma} N^{2} \iint\left(\widetilde{q}\left(\left[\Phi_{z}-\Phi_{w}\right]\right)-\widetilde{q}([z-w])\right) \widetilde{\mu}(\mathrm{d} z) \widetilde{\mu}(\mathrm{d} w) \leqslant \mathrm{O}\left(N^{2} t^{2}\right) \mathbb{E}\left(\sup _{z \in \mathbb{T}} \hat{\mu}\left(\left\{w:|[z-w]| \leqslant N^{\varepsilon} r\right\}\right)\right)$.

From the local density estimate for the Yukawa gas on the torus implied by Theorem 2.2, the above parenthesis is bounded by $\left(N^{\varepsilon} r\right)^{2}$ with high probability. We have therefore proved (6.17).

Equation (6.18) is a consequence of Proposition 2.5. Indeed, let $\left(\chi_{k}\right)_{k \geqslant 1}$ be a partition of unity in the sense that $\sum_{k} \chi_{k}(x)=1$ for any $x>r, \chi_{k}$ is supported on $\left[2^{k-1} r, 2^{k+1} r\right]$, and $\left\|\chi_{k}^{(n)}\right\|_{\infty} \leqslant C_{n}\left(2^{k} r\right)^{-n}$. We denote $f=q-\widetilde{q}$ and apply Proposition 2.5 to $G_{k}(z, w)=$ $f([z-w]) \chi_{k}(|[z-w]|)$ and $s=s_{k}=N^{-\varepsilon} 2^{k} r$, for some fixed small $\varepsilon>0$. For any $k$ such that $2^{k} r<\gamma N^{\varepsilon}$, we have

$$
\left|\nabla^{j} G_{k}(x, y)\right|=\mathrm{O}\left(|[x-y]|^{-j} \mathbb{1}_{|[x-y]| \in\left[2^{k-2} r, 2^{k+2} r\right]}\right)
$$

and the same estimate holds for $\left(G_{k}\right)_{\mathrm{B}_{s}}^{(j)}(x, y)$, defined in 2.24 . Proposition 2.5 gives

$$
N^{2} \iint G_{k}(z, w) \tilde{\mu}(\mathrm{d} z) \tilde{\mu}(\mathrm{d} w)=\mathrm{O}\left(N^{\varepsilon}\right)\left(\frac{1}{s_{k}^{4}} \sum_{j=0}^{p-1} s_{k}^{j}\left(s_{k} N^{\varepsilon}\right)^{2-j}+N^{2} s_{k}^{p}\left(s_{k} N^{\varepsilon}\right)^{2-p}\right)=\mathrm{O}\left(\frac{N^{3 \varepsilon}}{s_{k}^{2}}\right)
$$

where we chose $p=\lfloor 10 / \varepsilon\rfloor$. Summation of the above estimate over $1 \leqslant k \leqslant \log N$ gives

$$
N^{2} \iint G(z, w) \tilde{\mu}(\mathrm{d} z) \tilde{\mu}(\mathrm{d} w)=\mathrm{O}\left(\frac{N^{5 \varepsilon}}{r^{2}}\right)
$$


which proves $(6.18)$ for the term involving $f([z-w])$. The same estimate holds for the integral of $f\left(\left[\Phi_{z}-\Phi_{w}\right]\right)$, because for any fixed $m_{1}, m_{2}=\mathrm{O}(t)$ the function $(z, w) \mapsto f\left(\left[\Phi_{z}-\Phi_{w}\right]\right)$ has the same regularity properties as $(z, w) \mapsto f([z-w])(\Phi=\mathrm{Id}+t \phi$ for some function $\phi$ smooth on a scale 1$)$. This concludes the proof of 6.15 .

For equation (6.16), we first consider the averaging in the shift $(a, b)$ from $\Psi$ : denoting $|h|=\left(\left|h_{1}\right|,\left|h_{2}\right|\right)$, for any $h \in \mathbb{T}$ we have

$$
\begin{aligned}
\mathbb{E}_{(a, b)}\left(Y^{\gamma}([[h+(a, b)]-(a, b)])\right) & =Y^{\gamma}(|h|), \\
\mathbb{E}_{(a, b)}\left(Y^{\gamma}([h+(a, b)]-(a, b))\right) & =\left(1-\left|h_{1}\right|\right)\left(1-\left|h_{2}\right|\right) Y^{\gamma}(|h|)+\mathrm{O}\left(\mathrm{e}^{-N^{c}}\right),
\end{aligned}
$$

where the second equation comes from (6.14) and the fact that $Y^{\gamma}$ is essentially supported on $|x|<N^{\varepsilon} \gamma$. Equation (6.16) is therefore equivalent to

$\mathbb{E}^{\Phi} \mathbb{E}^{\gamma} N^{2} \iint\left(\left|\left(\Delta_{z}^{w}\right)_{1}+\right|\left(\Delta_{z}^{w}\right)_{2}|-|\left(\Delta_{z}^{w}\right)_{1}||\left(\Delta_{z}^{w}\right)_{2} \mid\right) Y^{\gamma}\left(\left|\Delta_{z}^{w}\right|\right) \tilde{\mu}(\mathrm{d} z) \tilde{\mu}(\mathrm{d} w)=\mathrm{O}\left(N^{\varepsilon}\right)\left(\frac{1}{\gamma^{4}}+\frac{\sqrt{N}}{t}\right)$

On the left side of the above equation, we would like to calculate the interaction after the $\Phi$ averaging. However, this expression is not a function of $[z-w]$, which would be convenient for our proof. We therefore perform an additional averaging over the torus: the above equation is equivalent to

$$
\mathbb{E}^{\gamma} N^{2} \iint K^{t}([z-w]) \tilde{\mu}(\mathrm{d} z) \tilde{\mu}(\mathrm{d} w)=\mathrm{O}\left(N^{\varepsilon}\right)\left(\frac{1}{\gamma^{4}}+\frac{\sqrt{N}}{t}\right)
$$

where $K^{t}(h)=\mathbb{E}^{\Phi} \int f\left(\Delta_{v}^{[v+h]}\right) m(\mathrm{~d} v)$ and $f(h)=\left(\left|h_{1}\right|+\left|h_{2}\right|-\left|h_{1} h_{2}\right|\right) Y^{\gamma}(h)$.

The proof of 6.22 is delicate, so before giving the technical details, we list below the main difficulties and ingredients.

(i) The function $K^{0}$ is smooth on $\mathbb{T}$ except on $h_{1}=0$ or $h_{2}=0$, as explained in Remark 6.5. This prevents a direct application of Proposition 2.5 and is the motivation for our averaging over $\Phi$.

(ii) The function $K^{t}$ now gained some smoothness in neighborhoods of $h_{1}=0$ and $h_{2}=0$ thanks to the convolution with the distribution of $t X$. For example, around $h_{1}=0, K^{t}$ is smooth on a scale $\left|t h_{2}\right|:$ for $k \geqslant 1, \partial_{h_{1}}^{k} K^{t}(h)=\mathrm{O}\left(\left|h_{1}\right|\left|t h_{2}\right|^{-k+1}\right)$, thanks to the definition of $\Phi_{1}$ in (6.7) and the asymptotics $t X s\left(h_{2}\right) \sim 2 \pi t X h_{2}$. Proposition 2.5 can now be applied for the function $K^{t}$, after some surgery removing some small singular set corresponding to the cross $\left\{\left|t h_{2}\right| \leqslant N^{-1 / 2}, h_{1} \leqslant N^{-1 / 2}\right\} \cup\left\{\left|t h_{1}\right| \leqslant N^{-1 / 2}, h_{2} \leqslant N^{-1 / 2}\right\}$.

We now implement the above outline. The function $K^{t}$ is a linear combination of the terms

$$
F_{j}^{t}(h)=\mathbb{E}^{\Phi} \int f_{j}\left(\Delta_{v}^{[v+h]}\right) m(\mathrm{~d} v)
$$

where $\Delta_{v}^{[v+h]}$ is defined in 6.13 and

$$
f_{1}(h)=\left|h_{1}\right| Y^{\gamma}(h), \quad f_{2}(h)=\left|h_{2}\right| Y^{\gamma}(h), f_{3}(h)=\left|h_{1} h_{2}\right| Y^{\gamma}(h) .
$$



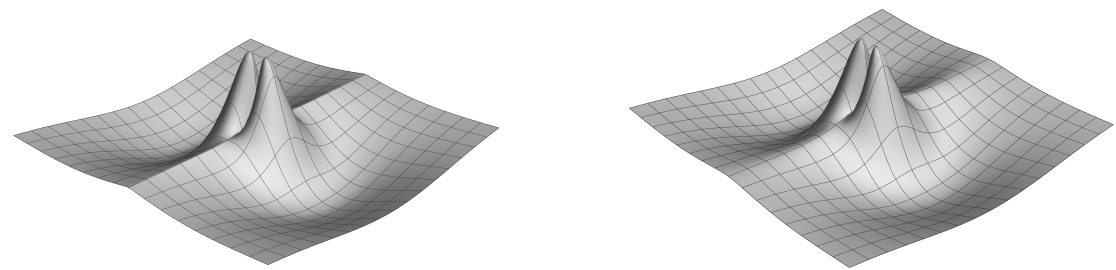

Figure 6.1. The functions $F_{1}^{0}$ (left) and $F_{1}^{t}$ (right) in $[-1 / 5,1 / 5]^{2}, \gamma=t=1 / 20$

We first bound the contribution from $F_{1}^{t}$ (and therefore $F_{2}^{t}$ by a similar argument), by exploiting its smoothness properties. Thus a calculation on $\left\{h_{1} \neq 0\right\}$ gives

$$
\begin{aligned}
& \left|\partial_{h_{1}} F_{1}^{0}(h)\right| \leqslant C\left(\frac{\left|h_{1}\right|}{|h|}+\left|Y^{\gamma}(h)\right|\right), \\
& \left|\partial_{h_{2}} F_{1}^{0}(h)\right| \leqslant C \frac{\left|h_{1}\right|}{|h|}, \\
& \left|\partial_{h_{1}}^{k_{1}} \partial_{h_{2}}^{k_{2}} F_{1}^{0}(h)\right| \leqslant \frac{C_{k_{1}, k_{2}}}{|h|^{k_{1}+k_{2}-1}}, \quad \text { if } k_{1}+k_{2} \geqslant 2 .
\end{aligned}
$$

Here we have used $\left|\partial_{h_{1}}^{k_{1}} \partial_{h_{2}}^{k_{1}} Y^{\gamma}(h)\right| \leqslant C_{k_{1}, k_{2}}|h|^{-\left(k_{1}+k_{2}\right)}$ and $\left[\Phi_{w}-\Phi_{z}\right]=[w-z]$ when $t=0$.

Recall that $\Phi$ is defined in (6.7) with $m_{1}, m_{2}$ given by independent random variables with distribution $t X$ with $X$ a compactly supported random variable of order one. Thus $\Delta_{v}^{[v+h]} \sim h$ with a distortion of order $t$, which makes the function $\left|\left(\Delta_{v}^{[v+h]}\right)_{1}\right|$ differentiable on $\left\{h_{1}=0\right\}$. With this in mind and explicitly writing $F_{1}^{t}$ with the definition 6.19 , a simple calculation and the Taylor expansion extends the estimate $(6.25)$ to

$$
\begin{aligned}
& \left|\partial_{h_{1}} F_{1}^{t}(h)\right| \leqslant C\left(\frac{\left|h_{1}\right|+\left|t h_{2}\right|}{|h|}+\left|Y^{\gamma}(h)\right|\right), \\
& \left|\partial_{h_{2}} F_{1}^{t}(h)\right| \leqslant C \frac{\left|h_{1}\right|+\left|t h_{2}\right|}{|h|}, \\
& \left|\partial_{h_{1}}^{k_{1}} \partial_{h_{2}}^{k_{2}} F_{1}^{t}(h)\right| \leqslant C_{k_{1}, k_{2}}\left(\frac{1}{|h|^{k_{1}+k_{2}-1}}+\frac{1}{\left|t h_{2}\right|^{k_{1}-1}\left|h_{2}\right|^{k_{2}}} \mathbb{1}_{\left|h_{1}\right|<t\left|h_{2}\right|, k_{1} \geqslant 1}\right)\left(k_{1}+k_{2} \geqslant 2\right) .
\end{aligned}
$$

For some mesoscopic scale $N^{-1 / 2+c} \leqslant r \ll t$, define a partition of unity $\mathbb{1}_{[0,1]}(x)=\sum_{i=0}^{n} \tilde{\chi}_{i}$ where $n$ is of order $\log N, \tilde{\chi}_{0}$ is supported on $[0,2 r], \chi_{i}$ is supported on $\left[2^{i-1} r, 2^{i+1} r\right]$, and $\left\|\chi_{i}^{(m)}\right\|_{\infty} \leqslant C_{m}\left(2^{i} r\right)^{-m}$. We define $F_{i j}^{t}(h)=F_{1}^{t}(h) \widetilde{\chi}_{|i|}\left(\left|h_{1}\right|\right) \widetilde{\chi}_{|j|}\left(\left|h_{2}\right|\right)$ for $|i|,|j| \leqslant n$. By symmetry, we only need to bound each $\iint F_{i j}^{t}$ in one quadrant: we now assume $0 \leqslant i, j \leqslant n$.

First, for $i=j=0$ (in fact for $i+j$ bounded), the local density estimate and $\left\|F_{i j}^{t}\right\|_{\infty}=$ $\mathrm{O}\left(r^{1+\varepsilon}\right)$ give

$$
N^{2} \mathbb{E}^{\gamma} \iint F_{i j}^{t}([z-w]) \tilde{\mu}(\mathrm{d} z) \tilde{\mu}(\mathrm{d} w)=\mathrm{O}\left(N^{\varepsilon}\right) N^{2} r^{3} .
$$

We now assume $i+j>0$.

For $2^{i}>t 2^{j}$ (in other words $\left|h_{1}\right|>t\left|h_{2}\right|$ ), (6.26) yields

$$
\left|\partial_{h_{1}}^{k_{1}} \partial_{h_{2}}^{k_{2}} F_{i j}^{t}(h)\right| \leqslant C_{k_{1}, k_{2}} \frac{1}{\max \left(2^{i} r, 2^{j} r\right)^{k_{1}+k_{2}-1}} .
$$


The area of the support of $F_{i j}$ is $\mathrm{O}\left(r^{2} 2^{i+j}\right)$. We proceed as in the proof of 6.21). We use Proposition 2.5 with the parameter $s$ in the Proposition chosen to be $\max \left(2^{i} r, 2^{j} r\right) N^{-\varepsilon}$. Thus (2.25) gives

$$
N^{2} \mathbb{E}^{\gamma} \iint F_{i j}^{t}([z-w]) \tilde{\mu}(\mathrm{d} z) \tilde{\mu}(\mathrm{d} w)=\mathrm{O}\left(N^{\varepsilon}\right)\left(\frac{1}{\gamma^{4}}+\frac{1}{\left(\max \left(2^{i} r, 2^{j} r\right)\right)^{4}}\right) \max \left(2^{i} r, 2^{j} r\right)\left(r^{2} 2^{i+j}\right) .
$$

Notice that the error term in 2.25) is negligible here by choosing $p \varepsilon>10$, say. We will often use this argument and from now no we will not repeat it in details.

After summation over $i, j$, we obtain

$$
\sum_{2^{i}>t 2^{j}} N^{2} \mathbb{E}^{\gamma} \iint F_{i j}^{t}([z-w]) \tilde{\mu}(\mathrm{d} z) \tilde{\mu}(\mathrm{d} w)=\mathrm{O}\left(N^{\varepsilon}\right)\left(\frac{1}{\gamma^{4}}+\frac{1}{r}\right) .
$$

For $i>0$ and $2^{i}<t 2^{j}\left(\left|h_{1}\right|<t\left|h_{2}\right|\right)$, from $(6.26)$ we have

$$
\left|\partial_{h_{1}}^{k_{1}} \partial_{h_{2}}^{k_{2}} F_{i j}^{t}(h)\right| \leqslant C_{k_{1}, k_{2}} \frac{1}{\left(t 2^{j} r\right)^{k_{1}+k_{2}-1}} .
$$

The area of the support of $F_{i j}$ is still of order $r^{2} 2^{i+j}$, so that Proposition 2.5 now yields

$$
N^{2} \mathbb{E}^{\gamma} \iint F_{i j}^{t}([z-w]) \tilde{\mu}(\mathrm{d} z) \tilde{\mu}(\mathrm{d} w)=\mathrm{O}\left(N^{\varepsilon}\right)\left(\frac{1}{\gamma^{4}}+\frac{1}{\left(t 2^{j} r\right)^{4}}\right)\left(t 2^{j} r\right)\left(r^{2} 2^{i+j}\right) .
$$

The contribution of such terms is therefore

$$
\sum_{2^{i}<t 2^{j}, i>0} N^{2} \mathbb{E}^{\gamma} \iint F_{i j}^{t}([z-w]) \tilde{\mu}(\mathrm{d} z) \tilde{\mu}(\mathrm{d} w)=\mathrm{O}\left(N^{\varepsilon}\right)\left(\frac{t^{2}}{\gamma^{4}}+\frac{1}{r t}\right) .
$$

For $i=0$ and $t 2^{j} r \geqslant N^{-1 / 2+\varepsilon}\left(\left|t h_{2}\right| \geqslant N^{-1 / 2+\varepsilon}\right)$, we have from 6.26

$$
\left|\partial_{h_{1}}^{k_{1}} \partial_{h_{2}}^{k_{2}} F_{i j}^{t}(h)\right| \leqslant C_{k_{1}, k_{2}} \frac{1}{\left(t 2^{j} r\right)^{k_{1}+k_{2}-1}}
$$

so that Proposition 2.5 gives

$$
N^{2} \mathbb{E}^{\gamma} \iint F_{0 j}^{t}([z-w]) \tilde{\mu}(\mathrm{d} z) \tilde{\mu}(\mathrm{d} w)=\mathrm{O}\left(N^{\varepsilon}\right)\left(\frac{1}{\gamma^{4}}+\frac{1}{\left(t 2^{j} r\right)^{4}}\right)\left(t 2^{j} r\right)\left(r^{2} 2^{j}\right),
$$

and therefore

$$
\sum_{N^{-1 / 2+\varepsilon}<t 2^{j} r} N^{2} \mathbb{E}^{\gamma} \iint F_{0 j}^{t}([z-w]) \tilde{\mu}(\mathrm{d} z) \tilde{\mu}(\mathrm{d} w)=\mathrm{O}\left(N^{\varepsilon}\right)\left(\frac{r t}{\gamma^{4}}+\frac{r N}{t}\right) .
$$

For the terms corresponding to $i=0, j>0$ and $t 2^{j} r \leqslant N^{-1 / 2+\varepsilon}$, we need one more decomposition. Let $r^{\prime}=N^{-1 / 2+\varepsilon^{\prime}} \ll r$, and decompose $F_{0 j}^{t}=A_{j}+B_{j}$ with

(i) $A_{j}$ supported on $\left\{\left|h_{1}\right|<2 r^{\prime}\right\} \cap\left\{2^{j-1} r<h_{2}<2^{j+1} r\right\},\left\|A_{j}\right\|_{\infty} \leqslant r^{\prime}$,

(ii) $B_{j}$ smooth, supported on $\left\{\left|h_{1}\right|<2 r\right\} \cap\left\{2^{j-1} r<h_{2}<2^{j+1} r\right\}$, satisfying $\left\|B_{j}\right\|_{\infty} \leqslant r$, and

$$
\left|\partial_{h_{1}}^{k_{1}} \partial_{h_{2}}^{k_{2}} B_{j}(h)\right| \leqslant C_{k_{1}, k_{2}}\left(\frac{r}{\left|h_{2}\right|^{k_{1}+k_{2}}}+\frac{r}{\left(r^{\prime}\right)^{k_{1}+k_{2}}} \mathbb{1}_{\left|h_{1}\right|<r^{\prime}}\right) .
$$


More explicitly, $A_{j}$ and $B_{j}$ can be constructed from $F_{0 j}$ as follows. Let $a \geqslant 0$ be smooth on $\mathbb{R}_{+}$, $a=1$ on $[0,1], a>0$ on $[1,2]$ and $a=0$ on $[2, \infty)$. Let $g \geqslant 0$ be a smooth, compactly supported function on $\mathbb{C}$ with $\int g=1$. Define

$$
g_{\eta}(z)=\frac{1}{\left(r^{\prime} a(|\eta|)\right)^{2}} g\left(\frac{z}{r^{\prime} a(|\eta|)}\right),
$$

with the convention $g_{\eta}=\delta_{0}$ when $|\eta|>2$, and

$$
B_{j}(z)=\left(F_{0 j} * g_{h_{2} / r^{\prime}}\right)(z), A_{j}=F_{0 j}-B_{j} .
$$

Then the functions $A_{j}$ and $B_{j}$ satisfy (i) and (ii): for example, note that that in the region $\left\{r^{\prime}<h_{1}<r\right\} \cap\left\{2^{j-1} r<h_{2}<2^{j+1} r\right\}, B \sim h_{1} Y^{\gamma}(h)$, and this function satisfies the estimates in (ii).

The function $A_{j}$ is supported on a domain of area $\mathrm{O}\left(2^{j} r r^{\prime}\right)$ and $\|A\|_{\infty} \leqslant r^{\prime}$, and the local density implied by Theorem 2.2 gives

$$
N^{2} \mathbb{E}^{\gamma} \iint A_{j}([z-w]) \tilde{\mu}(\mathrm{d} z) \tilde{\mu}(\mathrm{d} w)=\mathrm{O}\left(N^{1+2 \varepsilon} 2^{j} r\right)
$$

The contribution of all $A_{j}$ terms is therefore

$$
\sum_{j>0: t 2^{j} r \leqslant N^{-1 / 2+\varepsilon}} N^{2} \mathbb{E}^{\gamma} \iint A_{j}([z-w]) \tilde{\mu}(\mathrm{d} z) \tilde{\mu}(\mathrm{d} w)=\mathrm{O}\left(\frac{N^{1 / 2+3 \varepsilon}}{t}\right) .
$$

For the contribution from $B_{j}$, consider the following partition of $\mathbb{T}: 1=\sum_{-1 /(2 r) \leqslant a, b \leqslant 1 /(2 r)} \chi_{a b}$ where $\chi_{a b}$ is supported on a disk of radius $10 r$ around $(a r, b r)$, and $\left\|\chi_{a b}^{(n)}\right\|_{\infty} \leqslant C_{n} r^{-n}$. The contribution of $B_{j}$ is of order at most

$$
r^{-2} N^{2} \mathbb{E}^{\gamma} \iint \sum_{|a| \leqslant 5,2^{j-1} \leqslant b \leqslant 2^{j+1}} B_{j}([z-w]) \chi_{00}(z) \chi_{a b}(w) \tilde{\mu}(\mathrm{d} z) \tilde{\mu}(\mathrm{d} w) .
$$

Let $E$ be the event that all particles at distance $4 r$ from 0 are known. Then

$$
N^{2} \mathbb{E}^{\gamma} \iint B_{j}([z-w]) \chi_{00}(z) \chi_{a b}(w) \tilde{\mu}(\mathrm{d} z) \tilde{\mu}(\mathrm{d} w)=\mathbb{E}^{\gamma} \mathbb{E}^{\gamma}\left(\int f(z) \chi_{00}(z) N \tilde{\mu}(\mathrm{d} z) \mid E\right)
$$

where $f(z)=\int B\left([z-w] \chi_{a b}(w) N \tilde{\mu}(\mathrm{d} w)\right.$. By the local law Theorem 2.2, the set of $E$ such that

$$
\begin{aligned}
& f(z)=\mathrm{O}\left(N r^{3}\right), \\
& \nabla f(z)=\int \nabla\left(B\left([z-w] \chi_{a b}(w)\right) N \tilde{\mu}(\mathrm{d} w)=\mathrm{O}\left(N r^{2}\right),\right. \\
& \Delta f(z)=\int \Delta\left(B([z-w]) \chi_{a b}(w)\right) N \tilde{\mu}(\mathrm{d} w)=\mathrm{O}(N r),
\end{aligned}
$$

has measure at least $1-N^{-100}$. Using the (conditioned version of the) local law, Theorem 2.8 for $E$ in such a good set we therefore have

$$
\begin{aligned}
& \left|\mathbb{E}^{\gamma}\left(\int f(z) \chi_{00}(z) N \tilde{\mu}(\mathrm{d} z) \mid E\right)\right| \\
& \quad \leqslant\left(N r^{2}\left(\int\left|\nabla\left(f \chi_{00}\right)\right|^{2}+\frac{1}{\gamma^{2}} \int\left(f \chi_{00}\right)^{2}\right)\right)^{1 / 2}+N^{\varepsilon} r^{2}\left\|\Delta\left(f \chi_{00}\right)\right\|_{\infty}=\mathrm{O}\left(N^{3 / 2} r^{4}\right) .
\end{aligned}
$$


Hence the contribution of $B_{j}$ is at most

$$
N^{2} \mathbb{E}^{\gamma} \iint B_{j}([z-w]) \tilde{\mu}(\mathrm{d} z) \tilde{\mu}(\mathrm{d} w)=\mathrm{O}\left(N^{\varepsilon}\right) 2^{j} N^{3 / 2} r^{2}
$$

All $B_{j}$ terms are therefore bounded by

$$
\sum_{j>0: t 2^{j} r \leqslant N^{-1 / 2+\varepsilon}} N^{2} \mathbb{E}^{\gamma} \iint B_{j}([z-w]) \tilde{\mu}(\mathrm{d} z) \tilde{\mu}(\mathrm{d} w)=\mathrm{O}\left(N^{\varepsilon}\right) \frac{N r}{t} .
$$

Equations 6.27, 6.28, 6.29, 6.30, 6.31 and 6.32 show that $r=N^{-1 / 2+c}$ for arbitrarily small $c$ is the best choice. We therefore proved

$$
N^{2} \mathbb{E}^{\gamma} \iint F_{1}^{t}([z-w]) \tilde{\mu}(\mathrm{d} z) \tilde{\mu}(\mathrm{d} w)=\mathrm{O}\left(N^{\varepsilon}\right)\left(\frac{1}{\gamma^{4}}+\frac{\sqrt{N}}{t}\right) .
$$

The contribution from $F_{3}^{t}$ can be bounded following the same method, and the resulting estimate is smaller due to the extra small $\left|h_{2}\right|$ factor in $f_{3}$. Inserting the estimate 6.33 for $F_{i}^{t}, i=1,2,3$ into 6.23), we have completed the proof of 6.22 and thus 6.2.

6.4. Proof of estimate 6.3. By 6.14 we need to bound

$$
N^{2} \mathbb{E}^{\Phi} \mathbb{E}^{\gamma} \iint \mathcal{T}_{L}\left(\left[\Delta_{z}^{w}\right]\right) \tilde{\mu}(\mathrm{d} z) \tilde{\mu}(\mathrm{d} w), \quad L=L_{R}^{\gamma}+c
$$

where $c$ is an arbitrary constant. Without loss of generality, we choose $c=\log R-\log \gamma$, so that from 2.2 we have

$$
L(z)=\mathrm{O}(|z| / \gamma)
$$

for $|z| \ll \gamma$. Equation (6.34) is equivalent to

$$
N^{2} \mathbb{E}^{\gamma} \iint D^{t}([z-w]) \tilde{\mu}(\mathrm{d} z) \tilde{\mu}(\mathrm{d} w), \quad \text { where } D^{t}(h)=\mathbb{E}^{\Phi} \int \mathcal{T}_{L}\left(\Delta_{v}^{[v+h]}\right) m(\mathrm{~d} v),
$$

and we remind the reader that $\Phi$ depends on $t$ (note that we introduced an additional averaging over $v$ for the same reasons as in 6.22 ).

Our estimate of (6.36) is similar to 6.22), up to two differences. First, it is easier to bound 6.36 when the contributions from $g_{1}, g_{2}, g_{3}, g_{4}$ from 6.12 are isolated, but this cannot be performed directly: smoothness of $D_{t}$ across $h_{1}, h_{2}= \pm 1 / 2$ requires the combination of these four terms. In the first step, we therefore prove that the long-range contribution of $D_{t}$, which we will denote by $E_{t}$, is negligible (this problem was not present for $K^{t}$, which is essentially supported in a small neighborhood of 0 ).

Second, the most delicate decompositions of $K_{t}$ are not necessary for $D_{t}$ as we have the additional small factor 6.35 for the interaction at small distance.

First step. In this paragraph we prove that the contribution of the long range part in $D^{t}$ is of order

$$
N^{2} \mathbb{E}^{\gamma} \iint E^{t}([z-w]) \tilde{\mu}(\mathrm{d} z) \tilde{\mu}(\mathrm{d} w)=\mathrm{O}\left(N^{\varepsilon}\right)\left(\frac{1}{t^{3}}+\frac{1}{\gamma^{4}}\right)
$$

where $E^{t}(h)=\mathbb{E}^{\Phi} \int \mathcal{T}_{L}\left(\Delta_{v}^{[v+h]}\right)(1-\chi)\left(\Delta_{v}^{[v+h]}\right) m(\mathrm{~d} v)$ and $\chi$ is a smooth cutoff function equal to 1 on $\left|h_{1}\right|+\left|h_{2}\right| \leqslant 1 / 10,0$ on $\left|h_{1}\right|+\left|h_{2}\right|>1 / 5$. 
The function $\mathcal{T}_{L}(h)$ has discontinuous derivative on $\left\{h_{1}=0\right\} \cup\left\{h_{2}=0\right\}$, which imposes a detailed analysis around these axes. We first gain some order of magnitude of $\mathcal{T}_{L}(1-\chi)$ around these singularities by removing the following function,

$$
A(h)=\left(\tilde{\chi}\left(h_{1}\right)\left[\left(1-\left|h_{2}\right|\right) g_{1}(h)+\left|h_{2}\right| g_{3}(h)\right]+\tilde{\chi}\left(h_{2}\right)\left[\left(1-\left|h_{1}\right|\right) g_{1}(h)+\left|h_{1}\right| g_{2}(h)\right]\right)(1-\chi(h)),
$$

where $\tilde{\chi}$ is a smooth cutoff equal to 1 on $[0,1 / 200]$ and vanishing outside [0,1/100]. The function $A$ is smooth on $\mathbb{T}$ for the following two reasons. First, the function is smooth on $h_{1}=0$ because the following three estimates cannot be simultaneously satisfied: $\left|h_{1}\right|<1 / 1000, \tilde{\chi}\left(h_{2}\right) \neq 0$ and $(1-\chi)(h) \neq 0$. Similarly, the function is smooth on $h_{2}=0$. Second, $A$ is smooth on $h_{1}= \pm 1 / 2$ and $h_{2}= \pm 1 / 2$. Indeed, assume $-1 / 2 \leqslant h_{2}<1 / 2$ is fixed. Then $\left(1-\left|h_{1}\right|\right)\left((1-\chi) g_{1}\right)(h)+$ $\left|h_{1}\right|\left((1-\chi) g_{2}\right)(h)$ admits an obvious smooth extension to $h_{1} \in(0,1)$, and this extension is symmetric in a neighborhood of $h_{1}=1 / 2$, hence all its odd derivatives vanish there, so that $A$ is smooth at $h_{1}= \pm 1 / 2$. The same reasoning applies to $h_{2}= \pm 1 / 2$.

We define $\mathcal{A}(h)=\mathbb{E}^{\Phi} \int A\left(\Delta_{v}^{[v+h]}\right) m(\mathrm{~d} v)$. As $A$ is smooth at the scale of order one, from Proposition 2.5 with $t$ chosen to be $N^{-\varepsilon}$ and $p$ large enough, we obtain

$$
N^{2} \mathbb{E}^{\gamma} \iint \mathcal{A}([z-w]) \tilde{\mu}(\mathrm{d} z) \tilde{\mu}(\mathrm{d} w)=\mathrm{O}\left(\frac{N^{\varepsilon}}{\gamma^{4}}\right) .
$$

It thus remains to estimate $N^{2} \mathbb{E}^{\gamma} \iint H^{t}([z-w]) \tilde{\mu}(\mathrm{d} z) \tilde{\mu}(\mathrm{d} w)$ where $H^{t}=E^{t}-\mathcal{A}$. To understand the regularity properties of $H^{t}$, assume first that the distortion vanishes, i.e., $t=0$. We have $H^{0}=\mathcal{T}_{L}(1-\chi)-A$, so that $H^{0}$ is smooth on $\left\{h_{1} \neq 0\right\} \cap\left\{h_{2} \neq 0\right\}$, vanishes on $\left\{h_{1}=0\right\} \cup\left\{h_{2}=0\right\}$ (this is the purpose of removing the contribution from $A$ ) and satisfies (on the smoothness domain $\left.\left\{h_{1} \neq 0\right\} \cap\left\{h_{2} \neq 0\right\}\right)$

$$
\sup _{k_{1}+k_{2}=k}\left|\partial_{h_{1}}^{k_{1}} \partial_{h_{2}}^{k_{2}} H^{0}(h)\right| \leqslant C_{k}
$$

The distortion $t$ smooths the singularities on $\left\{h_{1}=0\right\} \cup\left\{h_{2}=0\right\}$ as follows:

$$
\left|H^{t}(h)\right| \leqslant C\left(t+\min \left(\left|h_{1}\right|,\left|h_{2}\right|\right)\right), \quad\left|\partial_{h_{1}}^{k_{1}} \partial_{h_{2}}^{k_{2}} H^{t}(h)\right| \leqslant C_{k_{1}, k_{2}}\left(1+\frac{\mathbb{1}_{\left|h_{2}\right|<t}}{t^{k_{2}-1}}+\frac{\mathbb{1}_{\left|h_{1}\right|<t}}{t^{k_{1}-1}}\right) .
$$

The above bounds are elementary after writing $H^{t}$ explicitly in terms of $g_{1}, g_{2}, g_{3}, g_{4}, \chi$ and $\tilde{\chi}$. It amounts to the observation that the function $r_{t}(x)=\mathbb{E}_{X}|x+t X|$ satisfies $\left|r_{t}^{(k)}(x)\right| \leqslant$ $C_{k}\left(1+\mathbb{1}_{|x|<2 t} t^{1-k}\right)$. Intuitively, $r_{t}(x)$ is a regularized absolute value function which is smooth at the scale $t$ and $r_{t}(x)=|x|$ for $|x| \geq 2 t$.

Let $\Omega_{t}=\left\{\left|h_{1}\right|<t\right\} \cup\left\{\left|h_{2}\right|<t\right\}$. Consider a partition of unity $1=\sum \chi_{i}$ on the torus with $\mathrm{O}(\log N)$ summands, $\chi_{0}$ with support on $\Omega_{t}, \chi_{i}(i>0)$ supported on $\left(2^{i+1} \Omega_{t}\right) \backslash\left(2^{i-1} \Omega_{t}\right)$, and the derivative bound $\left\|\chi_{i}^{(n)}\right\|_{\infty} \leqslant C_{n}\left(2^{i} t\right)^{-n}$ for all integer $n$. Note that for $H=H^{t} \chi_{i}$ we have $\left|\nabla^{j} H(x, y)\right|=\mathrm{O}\left(\left(2^{i} t\right)^{-j+1}\right)$, and the same estimate holds for $H_{s}^{(j)}$ when $s=\left(2^{i} t\right) N^{-\varepsilon}$. Moreover, $\left(2^{i+1} \Omega_{t}\right) \backslash\left(2^{i-1} \Omega_{t}\right)$ has area $\mathrm{O}\left(2^{i} t\right)$, so that Proposition 2.5 gives (take $p=\lfloor 10 / \varepsilon\rfloor$ )

$$
N^{2} \mathbb{E}^{\gamma} \iint H^{t}([z-w]) \chi_{i}([z-w]) \tilde{\mu}(\mathrm{d} z) \tilde{\mu}(\mathrm{d} w)=\mathrm{O}\left(N^{\varepsilon}\right)\left(\frac{1}{\left(2^{i} t\right)^{3}}+\frac{2^{i} t}{\gamma^{4}}\right), \quad \text { for } 2^{i} t<10 .
$$

Summation of the above equations over $i$ gives

$$
N^{2} \mathbb{E}^{\gamma} \iint H^{t}([z-w])(1-\chi)([z-w]) \tilde{\mu}(\mathrm{d} z) \tilde{\mu}(\mathrm{d} w)=\mathrm{O}\left(N^{\varepsilon}\right)\left(\frac{1}{t^{3}}+\frac{1}{\gamma^{4}}\right) .
$$

Equations 6.38 and 6.40 prove 6.37. 
Second step. In this paragraph we prove that the contribution of the short range is

$$
N^{2} \mathbb{E}^{\gamma} \iint U^{t}([z-w]) \tilde{\mu}(\mathrm{d} z) \tilde{\mu}(\mathrm{d} w)=\mathrm{O}\left(N^{\varepsilon}\right)\left(\frac{1}{\gamma^{4}}+\frac{N^{1 / 2}}{t}+\frac{N^{4 / 5}}{\gamma^{2 / 5}}\right),
$$

where $U^{t}(h)=\mathbb{E}^{\Phi} \int \mathcal{T}_{L}\left(\Delta_{v}^{[v+h]}\right) \chi\left(\Delta_{v}^{[v+h]}\right) m(\mathrm{~d} v)$. From our expression 6.11 for $\mathcal{T}_{L}$, we only need to bound $N^{2} \mathbb{E}^{\gamma} \iint U_{j}^{t}([z-w]) \tilde{\mu}(\mathrm{d} z) \tilde{\mu}(\mathrm{d} w)(1 \leqslant j \leqslant 3)$ where

$$
U_{j}^{t}(h)=\mathbb{E}^{\Phi} \int u_{j}\left(\Delta_{v}^{[v+h]}\right) m(\mathrm{~d} v),
$$

with

$$
u_{1}(h)=L(h) \chi(h), \quad u_{2}(h)=\left|h_{1}\right| L(h) \chi(h), \quad u_{3}(h)=\left|h_{1} h_{2}\right| L(h) \chi(h) .
$$

The terms above all involves $g_{1}$; the other ones involving $g_{2}, g_{3}, g_{4}$ can be bounded in an easier way, because $g_{2}, g_{3}, g_{4}$ are smooth on scale 1 with no singularity at 0 .

We first consider $U_{1}^{t}$. Let $N^{-1 / 2} \ll u \ll \gamma$ be some intermediate scale. Let $\chi$ be as before and define $\tilde{L}(h)=f_{1}(h) \chi(h / u)$. Then the local law, Theorem 2.2 and the bound 6.35 give

$$
N^{2} \mathbb{E}^{\gamma} \iint \tilde{U}_{1}^{t}([z-w]) \tilde{\mu}(\mathrm{d} z) \tilde{\mu}(\mathrm{d} w)=\mathrm{O}\left(\frac{N^{2+\varepsilon} u^{3}}{\gamma}\right), \quad \text { where } \tilde{U}_{1}^{t}(h)=\mathbb{E}^{\Phi} \int \tilde{L}\left(\Delta_{v}^{[v+h]}\right) m(\mathrm{~d} v) .
$$

On the other hand, the same reasoning as the paragraph from 6.20 to 6.21 gives

$$
N^{2} \mathbb{E}^{\gamma} \iint\left(U_{1}^{t}-\tilde{U}_{1}^{t}\right)([z-w]) \tilde{\mu}(\mathrm{d} z) \tilde{\mu}(\mathrm{d} w)=\mathrm{O}\left(\frac{N^{\varepsilon}}{u^{2}}\right)
$$

Optimization the parameter $u$ in both previous estimates shows that the contribution from $U_{1}^{t}$ is

$$
N^{2} \mathbb{E}^{\gamma} \iint U_{1}^{t}([z-w]) \tilde{\mu}(\mathrm{d} z) \tilde{\mu}(\mathrm{d} w)=\mathrm{O}\left(\frac{N^{4 / 5+\varepsilon}}{\gamma^{2 / 5}}\right) .
$$

We now consider the most delicate terms $U_{2}^{t}$ and $U_{3}^{t}$. We decompose $L_{R}^{\gamma}=Y^{R}-Y^{\gamma}+\mathrm{O}\left(e^{-N^{c}}\right)$, we can just replicate the proof for $F_{1}^{t}$ and $F_{3}^{t}$ and get the same estimates as (6.33) (note that, as for $U_{1}^{t}$, we could also have used the short range bound 6.35 for an improved but unnecessary estimate). This concludes the proof.

\section{Proof of Proposition 4.6: free energy lower bound}

In this section, we construct a trial state to give a correct lower bound for the free energy, and thus prove Proposition 4.6. Recall the assumptions of the proposition

$$
1 \gg \ell / b \gg\left(N b^{2}\right)^{-1 / 4}, \quad N^{-1 / 2+c} \ll \ell \ll b \ll N^{-\tau}, \quad \ell<R, \quad \tau=2 c / 5,
$$

which we will assume throughout this section.

7.1. The trial state and embedding of the torus. Throughout the proof of the lower bound the parameter $u \in[-b / 2, b / 2)^{2}$ is fixed arbitrarily, and all estimates will be uniform in the choice of $u$. To obtain a lower bound on the partition function, we first restrict the particle profile to $\overline{\mathbf{n}}$. For this, we define the indicator function

$$
\hat{\chi}(\mathbf{z})=\mathbb{1}\left(n_{B}(z)=\bar{n}_{B}\right) \prod_{\alpha} \mathbb{1}\left(n_{\alpha}(z)=\bar{n}_{\alpha}\right) \prod_{j} \mathbb{1}\left(z_{j} \in D \cup B\right)
$$


where $\mathbf{n}(\mathbf{z})$ is the particle profile of the configuration $\mathbf{z} \in \mathbb{C}^{N}$, i.e., $\mathbf{n}(\mathbf{z})=\left(n_{\alpha}(\mathbf{z})\right)$ where $n_{\alpha}(\mathbf{z})$ is the number of particles $z_{j} \in \alpha$ (with $\alpha$ either a bulk square or the boundary region $B$ ).

We then start with the trivial bound

$$
\frac{1}{\beta} \log \int \mathrm{e}^{-\beta H_{V}^{R}(\mathbf{z})} m(\mathrm{~d} \mathbf{z}) \geq \frac{1}{\beta} \log \int \mathrm{e}^{-\beta H_{V}^{R}(\mathbf{z})} \hat{\chi}(\mathbf{z}) m(\mathrm{~d} \mathbf{z}) .
$$

Next we break the permutation symmetry of the particles. We order the squares $\alpha$ arbitrarily as $\alpha_{1}, \alpha_{2}, \ldots$ and write $\tilde{\chi}(\mathbf{z})$ for $\hat{\chi}(\mathbf{z})$ multiplied with the indicator function of the event in which the particles $z_{1}, \ldots, z_{\bar{n}_{\alpha_{1}}}$ are in $\alpha_{1}$, the particles $z_{\bar{n}_{\alpha_{1}}+1}, \ldots, z_{\bar{n}_{\alpha_{1}}+\bar{n}_{\alpha_{2}}}$ are in $\alpha_{2}$, and so on. Then

$$
\frac{1}{\beta} \log \int \mathrm{e}^{-\beta H_{V}^{R}(\mathbf{z})} \hat{\chi}(\mathbf{z}) m(\mathrm{~d} \mathbf{z})=\frac{1}{\beta} \log \left(\begin{array}{c}
N \\
\mathbf{n}
\end{array}\right)+\frac{1}{\beta} \log \int \mathrm{e}^{-\beta H_{V}^{R}(\mathbf{z})} \tilde{\chi}(\mathbf{z}) m(\mathrm{~d} \mathbf{z})
$$

As in the lower bound for the torus in Section 3.6, to each bulk square $\alpha$, we associate a map

$$
\Psi_{\alpha}: \mathbb{T}^{(b)} \rightarrow \alpha .
$$

The main difference between these two settings is the choice of the embeddings $\Psi_{\alpha}$. We now choose $\Psi_{\alpha}$ as the re-centered version of the map defined by Corollary 6.2.

$$
\Psi_{\alpha}(v)=c(\alpha)+\Psi^{(b)}(v)=c(\alpha)+b \Psi(v / b) \quad\left(v \in \mathbb{T}^{(b)}\right)
$$

This choice of the maps will enter in this section only through the estimates given by Corollary 6.2 and the fact that $|\mathrm{d} \Psi|=1$.

The remaining set-up is parallel to that for the torus in Section 3.6. Let $\omega_{\alpha}$ be the measure of the Yukawa gas on $\mathbb{T}_{\alpha}^{n_{\alpha}}$, with density

$$
\omega_{\alpha}\left(\mathrm{d} \mathbf{v}^{\alpha}\right)=\frac{1}{Z_{\alpha}} \mathrm{e}^{-\beta \hat{H}_{\alpha}\left(\mathbf{v}^{\alpha}\right)} m\left(\mathrm{~d} \mathbf{v}^{\alpha}\right)
$$

where $\hat{H}_{\alpha}$ was defined in (4.14) as the energy of a torus Yukawa gas in $\mathbb{T}_{\alpha}$ with range $\ell$ (in principle, there is an external potential $Q(\alpha)$. Since it is a constant, we remove it). For the boundary, we take $\omega_{B}$ to be the measure under which the particles are independently distributed according to the equilibrium measure, i.e., $\omega_{B}=\left.\mu_{V}\right|_{B} ^{\otimes n_{B}}$ on $B$ and $\Psi_{B}: B \rightarrow B$ to be the identity map. With the fixed particle profile $\mathbf{n}=\overline{\mathbf{n}}$, the quasi-free approximation is the product measure $\boldsymbol{\omega}=\prod_{\alpha} \omega_{\alpha}$ (where the product also includes $\alpha=B$ ). Given the maps $\Psi_{\alpha}$, define $\Psi$ by

$$
\Psi: \prod_{\alpha} \mathbb{T}_{\alpha}^{n_{\alpha}} \times B^{n_{B}} \rightarrow \mathbb{C}^{N}, \quad \Psi(\{\mathbf{v}\})=\left(\left\{\Psi_{\alpha} \mathbf{v}^{\alpha}\right\}\right) \in \mathbb{C}^{N}
$$

In particular, $\Psi^{*} \boldsymbol{\omega}=\prod_{\alpha} \Psi_{\alpha}^{*} \boldsymbol{\omega}_{\alpha}$ is a measure on configurations of $N$ particles in $\mathbb{C}$. Under the map $\Psi_{\alpha}$, using that $\left|\mathrm{d} \Psi_{\alpha}\right|=1$, the measure $\omega_{\alpha}$ transforms to

$$
\frac{1}{Z_{\alpha}} \mathrm{e}^{-\beta \hat{H}_{\alpha}\left(\Psi_{\alpha}^{-1}(\mathbf{z})\right)} \prod_{i} \mathrm{~d} \Psi_{\alpha}^{-1}\left(z_{i}\right)=\frac{1}{Z_{\alpha}} \mathrm{e}^{-\beta \hat{H}_{\alpha}\left(\Psi_{\alpha}^{-1}(\mathbf{z})\right)} \prod_{i} \mathrm{~d} z_{i}
$$

where we write $\Psi_{\alpha}^{-1}(\mathbf{z})=\left(\Psi_{\alpha}^{-1}\left(z_{1}\right), \Psi_{\alpha}^{-1}\left(z_{2}\right), \ldots\right)$ if $\mathbf{z}=\left(z_{1}, z_{2}, \ldots\right)$. Thus defining $\hat{H}_{\Psi}(\mathbf{z})=$ $\sum_{\alpha} \hat{H}_{\alpha} \circ \Psi_{\alpha}^{-1}\left(\mathbf{z}^{\alpha}\right)$, by Jensen's inequality,

$$
\frac{1}{\beta} \log \int \mathrm{e}^{-\beta H_{V}^{R}(\mathbf{z})} \tilde{\chi}(\mathbf{z}) m(\mathrm{~d} \mathbf{z}) \geq \frac{1}{\beta} \log \int \mathrm{e}^{-\beta \hat{H}_{\Psi}(\mathbf{z})} \tilde{\chi}(\mathbf{z}) m(\mathrm{~d} \mathbf{z})+\mathbb{E}^{\Psi^{*} \boldsymbol{\omega}}\left(\hat{H}_{\Psi}-H_{V}^{R}\right) .
$$


Reversing the change of variables and averaging over the distribution of maps $\Psi$ with $|\mathrm{d} \Psi|=1$, whose expectation is denoted by $\mathbb{E}^{\Psi}$,

$$
\frac{1}{\beta} \log \int \mathrm{e}^{-\beta H_{V}^{R}} \tilde{\chi} \geq \frac{1}{\beta} \log \int \mathrm{e}^{-\beta \hat{H}_{\alpha}\left(\mathbf{v}^{\alpha}\right)} \prod_{\alpha} \mathrm{d} \mathbf{v}^{\alpha}+\Omega
$$

where $\Omega=\mathbb{E}^{\Psi} \mathbb{E}^{\boldsymbol{\omega}}\left(\hat{H}(\mathbf{v})-H_{V}^{R}(\Psi \mathbf{v})\right)$ with $\hat{H}(\mathbf{v})=\sum_{\alpha} \hat{H}_{\alpha}\left(\mathbf{v}^{\alpha}\right)$. We abbreviate by $\mathbb{E}^{\Psi} \mathbb{E}^{\boldsymbol{\omega}}$ the expectation $\mathbb{E}^{\Psi} \mathbb{E}^{\boldsymbol{\omega}}$ with $\boldsymbol{\omega}=\prod_{\alpha} \boldsymbol{\omega}_{\alpha}$, where $\boldsymbol{\omega}_{\alpha}$ is the measure of a Yukawa gas of range $\ell$ defined in the previous paragraph. Then, in summary, we need to estimate $\Omega=\Omega_{1}+\Omega_{2}$ where

$$
\Omega_{1}:=\mathbb{E}^{\Psi} \mathbb{E}^{\boldsymbol{\omega}}\left(\hat{H}(\mathbf{v})-H_{Q}^{\ell}(\Psi \mathbf{v})\right), \quad \Omega_{2}:=\mathbb{E}^{\Psi} \mathbb{E}^{\boldsymbol{\omega}}\left(H_{Q}^{\ell}(\Psi \mathbf{v})-H_{V}^{R}(\Psi \mathbf{v})\right)
$$

and we recall that $H_{Q}^{\ell}$ is the Yukawa energy of range $\ell$ with potential $Q$. Thus $\Omega_{1}$ is the error of a short range Yukawa gas in the quasifree approximation and is similar to that in (3.48) for the torus. The second term was absent for the torus because it was essentially handled by Lemma 3.3 at an earlier stage; the choice of $\ell \ll b$ in $(3.48)$ is the key reason that this term is much simpler on the torus than the current general setting. The control of this term requires a more careful choice of the maps $\Psi$.

7.2. Lower bound I: the short-range term $\Omega_{1}$. In the next two lemmas we estimate the shortrange contribution $\Omega_{1}$. These lemmas are analogous to Lemmas 3.123 .13 for the torus setting. Besides the density of the equilibrium measure is not constant, that there is a small contribution from the boundary, we also need precise estimate on $\Omega_{1}$ in the dependence of the parameters $\ell$ and $b$. With the current more sophisticated choice of the map $\Psi$ and the decoupling estimate, Corollary 6.2, we will be able to estimate $\Omega_{1}$ effectively.

First recall notation similar to that discussed around (3.49). As previously, $U_{b}^{\ell}$ is the Yukawa potential on the torus $\mathbb{T}^{(b)} \cong \mathbb{T}_{\alpha}$. Also, $\tilde{\mu}_{\alpha}=\hat{\mu}_{\alpha}-\mu_{\alpha}$ where $\mu_{\alpha}$ is the normalized uniform measure on $\mathbb{T}_{\alpha}$ and where $\hat{\mu}_{\alpha}$ is defined in $(3.49)$. We observe that, by construction, the expected empirical measure $\hat{\mu}$ under $\mathbb{E}^{\Psi} \mathbb{E}^{\boldsymbol{\omega}}$ in each square $\alpha$ is uniform with total mass $n_{\alpha} / N$ :

$$
N \mathbb{E}^{\Psi} \mathbb{E}^{\omega}\left(\left.\hat{\mu}\right|_{\alpha}\right)=n_{\alpha} \mu_{\alpha}, \quad \text { where } \mu_{\alpha}(d z)=b^{-2} \mathbb{1}_{z \in \alpha} m(\mathrm{~d} z) .
$$

The next lemma replaces Lemma 3.12 for the torus. Similarly as in 3.50 , we define

$$
E=\sum_{\alpha \subset D} n_{\alpha}^{2} \mathbb{E}^{\Psi} \mathbb{E}^{\omega} \iint_{\mathbb{T}_{\alpha} \times \mathbb{T}_{\alpha}}\left(U_{b}^{\ell}(v-w)-Y^{\ell}\left(\Psi_{\alpha}(v)-\Psi_{\alpha}(w)\right)\right) \tilde{\mu}_{\alpha}(\mathrm{d} v) \tilde{\mu}_{\alpha}(\mathrm{d} w) .
$$

We also write $\hat{H}_{D}=\sum_{\alpha \subset D} \hat{H}_{\alpha}$ and decompose $H_{Q}^{\ell}(\mathbf{z})$ into bulk and boundary contributions as

$$
H_{Q, D}^{\ell}(\mathbf{z})=N \sum_{j} Q\left(z_{j}\right) \mathbb{1}_{z_{j} \in D}+\sum_{j \neq k} Y^{\ell}\left(z_{j}-z_{k}\right) \mathbb{1}_{z_{j}, z_{k} \in D}, \quad H_{Q, B}^{\ell}(\mathbf{z})=H_{Q}^{\ell}(\mathbf{z})-H_{Q, D}^{\ell}(\mathbf{z})
$$

Lemma 7.1. Assume $1 \ll \ell \ll b$ and recall that $E$ is defined in $(7.12)$. Then

$$
\begin{aligned}
\mathbb{E}^{\Psi} \mathbb{E}^{\omega}\left(\hat{H}_{D}(\mathbf{u})-H_{Q, D}^{\ell}(\Psi \mathbf{u})\right) & =E+N^{\varepsilon} \mathrm{O}\left(N^{2}\left(\ell^{3}+b^{2} \ell^{2}\right)\right)\left\|\rho_{V}\right\|_{\infty, 2}^{2}, \\
N^{2} I_{B, Q}-\mathbb{E}^{\Psi} \mathbb{E}^{\omega}\left(H_{Q, B}^{\ell}(\mathbf{u})\right) & =N^{\varepsilon} \mathrm{O}\left(N^{2} \ell^{2} b\right)\left\|\nabla \rho_{V}\right\|_{\infty}+\mathrm{O}\left(\bar{n}_{B} \log N\right) .
\end{aligned}
$$

The proof of the above lemma occupies the remainder of this subsection. Before proceeding with the proof, we state the estimate for $E$ in the following lemma. 
Lemma 7.2. Assume the parameters $b$ and $\ell$ satisfy the condition (7.1). Then $E$ defined in 7.12 satisfies

$E=N^{\varepsilon} \mathrm{O}\left(b^{-2}\left(\left(N b^{2}\right)^{4 / 5} /(\ell / b)^{2 / 5}+(\ell / b)^{-4}\right)\right)=N^{\varepsilon} \mathrm{O}\left(N^{4 / 5} / \ell^{2 / 5}+b^{2} \ell^{-4}\right)=\mathrm{O}\left(N^{1-\tau}+N^{\varepsilon} b^{2} \ell^{-4}\right)$.

Proof. This is (6.4) of Corollary 6.2 and the fact that there are $\mathrm{O}\left(b^{-2}\right)$ bulk squares $\alpha$ according to 4.17 .

We now prove Lemma 7.1. The main error in 7.14$)$ is the one with the factor $N^{2}\left(\ell^{3}+b^{2} \ell^{2}\right)$, which is of order $b$ smaller than the main error term in the upper bound (5.4). The reason we gain an additional factor $b$ here, roughly speaking, is due to the fact that the leading error from the left side of a square is canceled by that from the right side provided that the densities of the two neighboring squares are the same. Since the density variation is of order $b$, the next order error carries an additional factor $b$. (A similar cancelation could have been obtained also in the upper bound (5.4). Since this refined estimate is not needed in this paper, we chose not to present it for the sake of simplicity.)

Proof of (7.14). Estimating $Q$ by $(4.9)$ and $\bar{n}_{\alpha}$ by (4.17), the difference of the contributions of the external potential is

$$
\begin{aligned}
& \left|\mathbb{E}^{\Psi} \mathbb{E}^{\boldsymbol{\omega}}\left[N \sum_{\alpha \subset D} \sum_{i}\left(Q\left(z_{i}\right)-Q(\alpha) \mathbb{1}_{z_{i} \in \alpha}\right)\right]\right|=\left|N \sum_{\alpha \subset D} n_{\alpha} \int(Q(z)-Q(\alpha)) \mu_{\alpha}(\mathrm{d} z)\right| \\
& \leqslant 4 \pi \ell^{2} N \sum_{\alpha \subset D} n_{\alpha}\left|\int\left(\rho_{V}(\alpha)-\rho_{V}(z)\right) \mu_{\alpha}(\mathrm{d} z)\right|+N \sum_{\alpha \subset D} \mathrm{O}\left(N b^{2}\left\|\rho_{V}\right\|_{\infty}\right) \mathrm{O}\left(N^{\varepsilon} \ell^{4}\left\|\nabla^{2} \rho_{V}\right\|_{\infty}\right) \\
& \leqslant \mathrm{O}\left(N^{2} b^{2} \ell^{2}\left\|\nabla \rho_{V}\right\|_{\infty}^{2}\right)+\mathrm{O}\left(N^{\varepsilon} N^{2} \ell^{4}\left\|\rho_{V}\right\|_{\infty}\left\|\nabla^{2} \rho_{V}\right\|_{\infty}\right) .
\end{aligned}
$$

To estimate the two-particle interactions, it suffices to show that

$$
\begin{aligned}
& \sum_{\alpha, \beta \subset D} \mathbb{E}^{\Psi} \mathbb{E}^{\omega}\left[\sum_{i \neq j} \mathbf{1}_{v_{i} \in \mathbb{T}_{\alpha}} \mathbf{1}_{v_{j} \in \mathbb{T}_{\beta}}\left(U_{\alpha}^{\ell}\left(v_{i}-v_{j}\right) \mathbb{1}_{\alpha=\beta}-Y^{\ell}\left(\Psi_{\alpha}\left(v_{i}\right)-\Psi_{\beta}\left(v_{j}\right)\right)\right)\right] \\
&=E+\mathrm{O}\left(N^{2} \ell^{3}\right)\left(\left\|\rho_{V}\right\|+\left\|\nabla \rho_{V}\right\|_{\infty}\right)^{2}
\end{aligned}
$$

The outline of the proof is analogous to that of (3.54) for the torus. Again, the contribution of the nonadjacent pairs of squares on the left-hand side is bounded by $\mathrm{O}\left(\mathrm{e}^{-c \ell / b}\right)=\mathrm{O}\left(N^{-\infty}\right)$. For any squares $\alpha, \beta$, define

$$
\bar{Y}_{\alpha \beta}=\iint_{\mathbb{T}_{\alpha} \times \mathbb{T}_{\beta}} Y^{\ell}\left(\Psi_{\alpha}(u)-\Psi_{\beta}(v)\right) \mu_{\alpha}(\mathrm{d} u) \mu_{\beta}(\mathrm{d} v)=\iint_{\alpha \times \beta} Y^{\ell}(u-v) \mu_{\alpha}(\mathrm{d} u) \mu_{\beta}(\mathrm{d} v) .
$$

Denoting by $\alpha \sim \beta$ that the squares $\alpha$ and $\beta$ are adjacent, exactly as in $(3.56)$, therefore

$$
\begin{gathered}
\sum_{\alpha, \beta \subset D} \mathbb{E}^{\Psi} \mathbb{E}^{\boldsymbol{\omega}}\left[\sum_{i \neq j} \mathbf{1}_{v_{i} \in \mathbb{T}_{\alpha}} \mathbf{1}_{v_{j} \in \mathbb{T}_{\beta}}\left(U_{b}^{\ell}\left(v_{i}-v_{j}\right) \mathbb{1}_{\alpha=\beta}-Y^{\ell}\left(\Psi_{\alpha}\left(v_{i}\right)-\Psi_{\beta}\left(v_{j}\right)\right)\right)\right] \\
=\sum_{\alpha \subset D} \bar{n}_{\alpha}^{2} \mathbb{E}^{\Psi} \mathbb{E}^{\boldsymbol{\omega}}\left[\iint_{\mathbb{T}_{\alpha} \times \mathbb{T}_{\alpha}}\left(U_{b}^{\ell}(v-w)-Y^{\ell}\left(\Psi_{\alpha}(v)-\Psi_{\alpha}(w)\right)\right) \hat{\mu}_{\alpha}(\mathrm{d} v) \hat{\mu}_{\alpha}(\mathrm{d} w)\right] \\
\quad-\sum_{\alpha \sim \beta} \bar{n}_{\alpha} \bar{n}_{\beta} \bar{Y}_{\alpha \beta}+\mathrm{O}\left(N^{-\infty}\right) .
\end{gathered}
$$


The difference between $E$ and the first term on the right-hand side of the above equation is

$$
\sum_{\alpha \subset D} \bar{n}_{\alpha}^{2}\left[\iint_{\mathbb{T}_{\alpha}^{2}} U_{b}^{\ell}(v-w) \mu_{\alpha}(\mathrm{d} v) \mu_{\alpha}(\mathrm{d} w)-\iint_{\alpha^{2}} Y^{\ell}(v-w) \mu_{\alpha}(\mathrm{d} v) \mu_{\alpha}(\mathrm{d} w)\right],
$$

where we have used that $\tilde{\mu}_{\alpha}=\hat{\mu}_{\alpha}-\mu_{\alpha}$. For the squares $\alpha \subset D$ not touching the boundary, we use the cancellation (7.21) below and for the squares touching the boundary instead the weaker estimate 7.22 . By these estimates, and summing over $\alpha$ using that there $\mathrm{O}\left(b^{-2}\right)$ squares $\alpha$ not touching a boundary square and $\mathrm{O}\left(b^{-1}\right)$ squares touching the boundary, it follows that the last display equals

$$
\sum_{\alpha \subset D} \sum_{\beta: \beta \sim \alpha} \bar{n}_{\alpha} \bar{n}_{\beta} \bar{Y}_{\alpha \beta}+\mathrm{O}\left(N^{2} \ell^{3}\right)\left(\left\|\rho_{V}\right\|_{\infty}+\left\|\nabla \rho_{V}\right\|_{\infty}\right)^{2}
$$

This proves (7.17).

The following lemma replaces Lemma 3.14 for the torus. The argument requires more care since we here do not have $\bar{n}_{\alpha}=\bar{n}_{\beta}$.

Lemma 7.3. Assume that $b \gg \ell$. For any bulk square $\alpha$ whose neighboring squares do not touch the boundary region $B$,

$$
\begin{array}{r}
\left.\bar{n}_{\alpha}^{2}\left[\iint_{\mathbb{T}_{\alpha} \times \mathbb{T}_{\alpha}} U^{\ell}(v-w) \mu_{\alpha}(\mathrm{d} v) \mu_{\alpha}(\mathrm{d} w)-\iint_{\alpha \times \alpha} Y^{\ell}(v-w)\right) \mu_{\alpha}(\mathrm{d} v) \mu_{\alpha}(\mathrm{d} w)\right]-\sum_{\beta \sim \alpha} \bar{n}_{\alpha} \bar{n}_{\beta} \bar{Y}_{\alpha \beta} \\
=\mathrm{O}\left(N^{2} b^{2} \ell^{3}\left\|\rho_{V}\right\|_{\infty}\left\|\nabla \rho_{V}\right\|_{\infty}\right)+\mathrm{O}\left(N^{-\infty}\right) .
\end{array}
$$

For all other squares $\alpha$, we still have

$$
\begin{array}{r}
\left.\bar{n}_{\alpha}^{2}\left[\iint_{\mathbb{T}_{\alpha} \times \mathbb{T}_{\alpha}} U^{\ell}(v-w) \mu_{\alpha}(\mathrm{d} v) \mu_{\alpha}(\mathrm{d} w)-\iint_{\alpha \times \alpha} Y^{\ell}(v-w)\right) \mu_{\alpha}(\mathrm{d} v) \mu_{\alpha}(\mathrm{d} w)\right]-\sum_{\beta \sim \alpha} \bar{n}_{\alpha} \bar{n}_{\beta} \bar{Y}_{\alpha \beta} \\
=\mathrm{O}\left(N^{2} b \ell^{3}\right)\left\|\rho_{V}\right\|_{\infty}^{2}
\end{array}
$$

Proof. For any fixed square $\alpha$ of side length $b \gg l$, using that contributions for distances $\gg \ell$ are negligible, by unfolding the periodized interaction we have

$$
\int_{\alpha} \int_{\alpha} U^{\ell}(u-v) m(\mathrm{~d} u) m(\mathrm{~d} v)=\int_{\alpha} \int_{\cup_{\beta \sim \alpha} \beta \cup \alpha} Y^{\ell}(z-w) m(\mathrm{~d} u) m(\mathrm{~d} v)+\mathrm{O}\left(N^{-\infty}\right),
$$

and thus

$$
\iint_{\alpha^{2}}\left(U^{\ell}(u-v)-Y^{\ell}(u-v)\right) \mu_{\alpha}(\mathrm{d} u) \mu_{\alpha}(\mathrm{d} v)=\sum_{\beta \sim \alpha} \iint_{\alpha \times \beta} Y^{\ell}(z-w) \mu_{\alpha}(\mathrm{d} z) \mu_{\beta}(\mathrm{d} w)+\mathrm{O}\left(N^{-\infty}\right) .
$$

Therefore the left-hand side of (7.21) equals

$$
\bar{n}_{\alpha} \sum_{\beta: \beta \sim \alpha}\left(\bar{n}_{\alpha}-\bar{n}_{\beta}\right) \iint_{\alpha \times \beta} Y^{\ell}(z-w) \mu_{\alpha}(\mathrm{d} z) \mu_{\beta}(\mathrm{d} w)+\mathrm{O}\left(N^{-\infty}\right) .
$$

Note that for $\alpha \neq \beta$,

$$
\iint_{\alpha \times \beta} Y^{\ell}(z-w) \mu_{\alpha}(\mathrm{d} z) \mu_{\beta}(\mathrm{d} w)=\mathrm{O}\left(b^{-2}\right) \mathrm{O}\left(\ell b^{-1}\right) \sup _{z \in \alpha} \int Y^{\ell}(z-w) m(\mathrm{~d} w)=\mathrm{O}\left(b^{-3} \ell^{3}\right) .
$$


Using $\left|\bar{n}_{\alpha}-\bar{n}_{\beta}\right|=\mathrm{O}\left(N b^{3}\right)\left\|\nabla \rho_{V}\right\|_{\infty}$ and $\bar{n}_{\alpha}=\mathrm{O}\left(N b^{2}\right)\left\|\rho_{V}\right\|_{\infty}$, the claim (7.21) follows.

For the boundary squares, we do not use any cancellation between the difference of $U^{\ell}$ and $Y^{\ell}$ and $\bar{Y}$ in (7.21), but we still use the cancellation between $U^{\ell}$ and $Y^{\ell}$. Analogously to the above, the difference between $U^{\ell}$ and $Y^{\ell}$ and the $\bar{Y}$ terms are each bounded by

$$
\mathrm{O}\left(N b^{2}\right)^{2} \mathrm{O}\left(b^{-3} \ell^{3}\right)\left\|\rho_{V}\right\|_{\infty}^{2}=\mathrm{O}\left(N^{2} b \ell^{3}\right)\left\|\rho_{V}\right\|_{\infty}^{2} .
$$

This completes the proof.

Proof of 7.15 . By definition, we have

$$
\mathbb{E}^{\Psi} \mathbb{E}^{\boldsymbol{\omega}}\left[H_{Q, B}^{\ell}\right]=\mathbb{E}^{\Psi} \mathbb{E}^{\boldsymbol{\omega}}\left[\sum_{i \neq j} Y^{\ell}\left(z_{i}-z_{j}\right) \mathbb{1}_{z_{i}, z_{j} \in B}+N \sum_{z_{i} \in B} Q\left(z_{j}\right)+2 \sum_{z_{i} \in D} \sum_{z_{j} \in B} Y^{\ell}\left(z_{i}-z_{j}\right)\right] .
$$

Moreover, by definition of the expectation $\mathbb{E}^{\Psi} \mathbb{E}^{\omega}$, the particles in $B$ are distributed independently according to the restriction of the equilibrium measure $\mu_{V}$. If the particles in $D$ were also distributed independently according to the equilibrium measure, the above right-hand side would be $N^{2} I_{Q, B}+\mathrm{O}\left(\bar{n}_{B} \log N\right)$, with the error term $\mathrm{O}\left(\bar{n}_{B} \log m(B)\right)=\mathrm{O}\left(\bar{n}_{B} \log N\right)$ resulting from the inclusion of the diagonal $i=j$ in the first sum. In reality, the particles in $D$ are distributed according to the periodic Yukawa gas in the squares $\alpha$; under this measure the expected empirical measure is uniform on the squares $\alpha$ with constant density $\bar{n}_{\alpha} / N$; we may replace this constant density in the bulk squares by the density of the equilibrium measure with an error $\mathrm{O}\left(N n_{B} \ell^{2} b b^{\prime}\right)\left\|\nabla \rho_{V}\right\|_{\infty}=\mathrm{O}\left(N n_{B} \ell^{2} b\right)\left\|\nabla \rho_{V}\right\|_{\infty}$. In summary, we have

$$
\mathbb{E}^{\Psi} \mathbb{E}^{\boldsymbol{\omega}}\left[H_{Q, B}^{\ell}\right]=N^{2} I_{Q, B}+\mathrm{O}\left(N^{2} \ell^{2} b\right)\left\|\nabla \rho_{V}\right\|_{\infty}+\mathrm{O}\left(\bar{n}_{B} \log N\right)
$$

as claimed.

7.3. Lower bound II: the long-range term $\Omega_{2}$ and conclusion. In the next lemma we estimate the term $\Omega_{2}$. It is in this estimate where the randomness of the $\Psi_{\alpha}$ enters in an essential way through the decoupling estimate, 6.5 of Corollary 6.2. We recall the decomposition $H_{Q}^{\ell}=H_{Q, D}^{\ell}+H_{Q, B}^{\ell}$ of the energy into bulk and boundary part from $(7.13)$ and decompose $H_{V}^{R}$ analogously.

Lemma 7.4. Assume the parameters $b$ and $\ell$ satisfy the condition (7.1) and recall $K_{R}^{\ell}$ from 4.5. Then

$\Omega_{2}=N \log (R / \ell)+N^{2} K_{R}^{\ell}+N^{\varepsilon} \mathrm{O}\left(N^{1-\tau}+b^{2} \ell^{-4}\right)+\mathrm{O}\left(N^{2} b^{4}\right)\|\nabla \rho\|_{\infty, 2}^{2}+\mathrm{O}\left((\log N) b^{-2}+n_{B} \log N\right)$.

More precisely, with $\mathrm{O}\left(N^{1-\tau}\right)=N^{\varepsilon} \mathrm{O}\left(N^{4 / 5} / \ell^{2 / 5}\right)$, we have

$$
\begin{aligned}
& \mathbb{E}^{\Psi} \mathbb{E}^{\boldsymbol{\omega}}\left(H_{Q, D}^{\ell}(\Psi \mathbf{v})\right.\left.-H_{V, D}^{R}(\Psi \mathbf{v})\right)-N \log (R / \ell)-N^{2} K_{R}^{\ell} \\
&=N^{\varepsilon} \mathrm{O}\left(N^{1-\tau}+b^{2} \ell^{-4}\right)+N^{\varepsilon} \mathrm{O}\left(N^{2} b^{4}\right)\|\nabla \rho\|_{\infty, 2}^{2}+\mathrm{O}\left((\log N) b^{-2}\right), \\
& \mathbb{E}^{\Psi} \mathbb{E}^{\boldsymbol{\omega}}\left(H_{Q, B}^{\ell}(\Psi \mathbf{v})-H_{V, B}^{R}(\Psi \mathbf{v})\right)=\mathrm{O}\left((\log N) b^{-2}+n_{B} \log N\right) .
\end{aligned}
$$

Assuming this Lemma, we can now prove Proposition 4.6.

Proof of Proposition 4.6. By 7.3-7.10,

$$
\begin{aligned}
& \frac{1}{\beta} \log \int \mathrm{e}^{-\beta H_{V}^{R}(\mathbf{z})} m(\mathrm{~d} \mathbf{z}) \\
& \geqslant \frac{1}{\beta} \log \left(\begin{array}{l}
N \\
\mathbf{n}
\end{array}\right)+\sum_{\alpha \subset D} \frac{1}{\beta} \log \int \mathrm{e}^{-\beta \hat{H}_{\alpha}\left(\mathbf{u}^{\alpha}\right)} \mathrm{d} \mathbf{u}^{\alpha}+\frac{1}{\beta} \log \int \mathrm{e}^{-\beta \hat{H}_{B}\left(\mathbf{u}^{B}\right)} \mathrm{d} \mathbf{u}^{B}+\Omega_{1}+\Omega_{2}
\end{aligned}
$$


where the combinatorial factor $\frac{1}{\beta} \log \left(\begin{array}{l}N \\ \mathbf{n}\end{array}\right)$ arises analogously as in $(3.41)$. By definition, the first three terms on the right-hand side give $F(\overline{\mathbf{n}})$. By Lemmas 7.1 and 7.4 the last two terms on the right-hand side, $\Omega_{1}+\Omega_{2}$, contribute the explicit terms $N \log (R / \ell)+N^{2} K_{R}^{\ell}$ as well as the error terms in the statement of the proposition.

The rest of this section is devoted to a proof of Lemma 7.4. We start with a proof of 7.24). Proof of (7.24). We start with (4.7), which implies that $\mathbb{E}^{\Psi} \mathbb{E}^{\omega}\left(H_{Q}^{\ell} \circ \Psi-H_{V}^{R} \circ \Psi\right)-N \log (R / \ell)-N^{2} K_{R}^{\ell}=-N^{2} \mathbb{E}^{\Psi} \mathbb{E}^{\omega}\left(\mathbf{L}_{R}^{\ell} \circ \Psi\right)=-N^{2} \mathbb{E}^{\Psi} \mathbb{E}^{\omega} \sum_{\alpha, \beta}\left(\Omega_{\alpha \beta} \circ \Psi\right)$,

where we define (note that $\Omega_{\alpha \beta}$ should not be confused with $\Omega_{1}$ and $\Omega_{2}$ )

$$
\Omega_{\alpha \beta}(\mathbf{z})=\iint_{\alpha \times \beta} L_{R}^{\ell}(v-w) \tilde{\mu}^{\mathbf{z}}(\mathrm{d} v) \tilde{\mu}^{\mathbf{z}}(\mathrm{d} w) ;
$$

here $\tilde{\mu}^{\mathbf{z}}=\hat{\mu}^{\mathbf{z}}-\mu_{V}^{R}$ and we have made its dependence on $\mathbf{z} \in \mathbb{C}^{N}$ through the empirical measure $\hat{\mu}=\hat{\mu}^{\mathbf{z}}$ explicit. Recall $\Psi$ from (7.7) and note that $\Omega_{\alpha \beta} \circ \Psi$ is a function on $\prod_{\alpha} \mathbb{T}_{\alpha}^{n_{\alpha}}$. For any $\mathbf{v} \in \prod_{\alpha} \mathbb{T}_{\alpha}^{n_{\alpha}}$, denote by $\hat{\mu}_{\alpha}^{\mathbf{v}}(\mathrm{d} v)=\bar{n}_{\alpha}^{-1} \sum_{j: v_{j} \in \mathbb{T}_{\alpha}} \delta_{v_{j}}(\mathrm{~d} v)$ the normalized empirical measure on $\mathbb{T}_{\alpha}$ as in (3.49). We also denote by $\mu_{\alpha}(\mathrm{d} v)=b^{-2} m(\mathrm{~d} v)$ the normalized uniform measure on $\mathbb{T}_{\alpha}$, and set $\tilde{\mu}_{\alpha}=\hat{\mu}_{\alpha}-\mu_{\alpha}$. We rewrite $\Omega_{\alpha \beta} \circ \Psi$ as

$$
\begin{aligned}
\Omega_{\alpha \beta}(\Psi(\mathbf{v}))=\iint_{\mathbb{T}_{\alpha} \times \mathbb{T}_{\beta}} L_{R}^{\ell}\left(\Psi_{\alpha}(v)-\Psi_{\beta}(w)\right) & {\left[\frac{\bar{n}_{\alpha}}{N} \hat{\mu}_{\alpha}^{\mathbf{v}}(\mathrm{d} v)-b^{2} \rho_{V}\left(\Psi_{\alpha}(v)\right) \mu_{\alpha}(\mathrm{d} v)\right] } \\
\times & {\left[\frac{\bar{n}_{\beta}}{N} \hat{\mu}_{\beta}^{\mathbf{v}}(\mathrm{d} w)-b^{2} \rho_{V}\left(\Psi_{\beta}(w)\right) \mu_{\beta}(\mathrm{d} w)\right], }
\end{aligned}
$$

where we changed variables $v \rightarrow \Psi_{\alpha}(v)$ and used $(6.9)$. We then rewrite

$$
\left[\bar{n}_{\alpha} \hat{\mu}_{\alpha}^{\mathbf{v}}(\mathrm{d} v)-N b^{2} \rho_{V}\left(\Psi_{\alpha}(v)\right) \mu_{\alpha}(\mathrm{d} v)\right]=\bar{n}_{\alpha} \tilde{\mu}_{\alpha}^{\mathbf{v}}(\mathrm{d} v)-\left[N b^{2} \rho_{V}\left(\Psi_{\alpha}(v)\right)-\bar{n}_{\alpha}\right] \mu_{\alpha}(\mathrm{d} v) .
$$

Using that $\mathbb{E}^{\omega} \tilde{\mu}_{\alpha}^{\mathbf{v}}(\mathrm{d} v)=0$ to see that the cross terms between the $\tilde{\mu}_{\alpha}^{\mathbf{v}}$ and $\mu_{\beta}$ or $\tilde{\mu}_{\beta}^{\mathrm{v}}$ terms vanish. This gives

$$
\begin{aligned}
& N^{2} \mathbb{E}^{\Psi} \mathbb{E}^{\omega} \Omega_{\alpha \beta}=\mathbf{1}_{\alpha=\beta} \bar{n}_{\alpha} \bar{n}_{\beta} \mathbb{E}^{\Psi} \mathbb{E}^{\omega} \iint_{\mathbb{T}_{\alpha} \times \mathbb{T}_{\beta}} L_{R}^{\ell}\left(\Psi_{\alpha}(v)-\Psi_{\beta}(w)\right) \tilde{\mu}_{\alpha}^{\mathrm{v}}(\mathrm{d} v) \tilde{\mu}_{\beta}^{\mathrm{v}}(\mathrm{d} w) \\
+ & N^{2} \mathbb{E}^{\Psi} \mathbb{E}^{\omega} \iint_{\mathbb{T}_{\alpha} \times \mathbb{T}_{\beta}} L_{R}^{\ell}\left(\Psi_{\alpha}(v)-\Psi_{\beta}(w)\right)\left[\rho_{V}\left(\Psi_{\alpha}(v)\right)-\frac{\bar{n}_{\alpha}}{N b^{2}}\right] m(\mathrm{~d} v)\left[\rho_{V}\left(\Psi_{\beta}(w)\right)-\frac{\bar{n}_{\beta}}{N b^{2}}\right] m(\mathrm{~d} w) .
\end{aligned}
$$

The proof is completed by bounding the sums of the two term in (7.31) over $\alpha, \beta$. The first term with $\alpha=\beta$ is the key difficulty requiring the sophisticated averaging over $\Psi$. Indeed, by 6.5) of Corollary 6.2 and using that there are $\mathrm{O}\left(b^{-2}\right)$ bulk squares $\alpha$, we have

$$
\begin{aligned}
& \sum_{\alpha \subset D} \bar{n}_{\alpha}^{2} \mathbb{E}^{\Psi} \mathbb{E}^{\omega} \iint_{\mathbb{T}_{\alpha} \times \mathbb{T}_{\alpha}} L_{R}^{\ell}\left(\Psi_{\alpha}(v)-\Psi_{\alpha}(w)\right) \tilde{\mu}_{\alpha}^{\mathbf{v}}(\mathrm{d} v) \tilde{\mu}_{\alpha}^{\mathbf{v}}(\mathrm{d} w) \\
&=N^{\varepsilon} b^{-2} \mathrm{O}\left(\left(N b^{2}\right)^{4 / 5} /(\ell / b)^{2 / 5}+b^{4} \ell^{-4}\right)=N^{\varepsilon} \mathrm{O}\left(N^{4 / 5} / \ell^{2 / 5}+b^{2} \ell^{-4}\right)
\end{aligned}
$$

as needed. The sum over $\alpha, \beta$ of the second term on the right-hand side of $(7.31)$ is bounded as needed in Lemma 7.5 stated below. 
In the statement of the following lemma, a naive bound of the left-hand side is $N^{2} b^{2}$. We gain an extra factor $b$ for each integration variable due to the cancellation of the integrand, and thus obtain the resulting stronger estimate.

Lemma 7.5. Assume the parameters $b$ and $\ell$ satisfy the condition (7.1). Then we have

$$
\begin{array}{r}
N^{2} \sum_{\alpha, \beta} \mathbb{E}^{\Psi} \mathbb{E}^{\omega} \iint_{\mathbb{T}_{\alpha} \times \mathbb{T}_{\beta}} L_{R}^{\ell}\left(\Psi_{\alpha}(v)-\Psi_{\beta}(w)\right)\left[\rho_{V}\left(\Psi_{\alpha}(v)\right)-\frac{\bar{n}_{\alpha}}{N b^{2}}\right] m(\mathrm{~d} v)\left[\rho_{V}\left(\Psi_{\beta}(w)\right)-\frac{\bar{n}_{\beta}}{N b^{2}}\right] m(\mathrm{~d} w) \\
=N^{\varepsilon} \mathrm{O}\left(N^{2} b^{4}\right)\left\|\nabla \rho_{V}\right\|_{\infty, 3}^{2}+\mathrm{O}\left((\log N) b^{-2}\right)
\end{array}
$$

where the sum is over bulk squares $\alpha, \beta$.

Proof. By changing variables, the claim is equivalent to

$$
\begin{array}{r}
N^{2} \sum_{\alpha, \beta \subset D} \mathbb{E}^{\Psi} \mathbb{E}^{\omega} \iint_{\alpha \times \beta} L_{R}^{\ell}(z-w)\left[\rho_{V}(z)-\frac{\bar{n}_{\alpha}}{N b^{2}}\right] m(\mathrm{~d} z)\left[\rho_{V}(w)-\frac{\bar{n}_{\beta}}{N b^{2}}\right] m(\mathrm{~d} w) \\
=N^{\varepsilon} \mathrm{O}\left(N^{2} b^{4}\right)\left\|\nabla \rho_{V}\right\|_{\infty, 3}^{2}+\mathrm{O}\left((\log N) b^{-2}\right) .
\end{array}
$$

By definition of $\bar{n}_{\alpha}$ we have $\left|\bar{n}_{\alpha}-N \mu_{V}(\alpha)\right| \leqslant 1$, and thus (recalling that $\mu_{V}$ has density $\rho_{V}$ )

$$
\rho_{V}(z)-\frac{\bar{n}_{\alpha}}{N b^{2}}=\rho_{V}(z)-\frac{\mu_{V}(\alpha)}{b^{2}}+O\left(\frac{1}{N b^{2}}\right)=\frac{1}{b^{2}} \int_{\alpha} m(\mathrm{~d} \zeta)\left(\rho_{V}(z)-\rho_{V}(\zeta)\right) \mathrm{d} \zeta+O\left(\frac{1}{N b^{2}}\right)
$$

as well as

$$
\rho_{V}(w)-\frac{\bar{n}_{\alpha}}{N b^{2}}=\frac{1}{b^{2}} \int_{\alpha} m(\mathrm{~d} \xi)\left(\rho_{V}(w)-\rho_{V}(\xi)\right) \mathrm{d} \xi+O\left(\frac{1}{N b^{2}}\right) .
$$

We will use these bounds below and also bound $L_{R}^{\ell}(z-w)$ by $\mathrm{O}(\log \ell)=\mathrm{O}(\log N)$ in the integral.

We first consider the diagonal terms $\alpha=\beta$ on the left-hand side of (7.34). We claim that the contribution of each such term is $\mathrm{O}(\log N)\left[N^{2} b^{6}\left\|\nabla \rho_{V}\right\|_{\infty}^{2}+1\right]$. To see this, note that the factor $\log N$ is due to $L_{R}^{\ell}$, and a factor $b^{2}$ arises from each of the integration of $z$ and $w$. The first terms on the right-hand sides of (7.35)-(7.36) contribute a factor $b\left\|\nabla \rho_{V}\right\|_{\infty}$ each from bounding $\left[\rho_{V}(z)-\rho(\zeta)\right]$ respectively $\left[\rho_{V}(w)-\rho(\xi)\right]$, while the error term are of order $1 /\left(N b^{2}\right)$. Since there are $\mathrm{O}\left(b^{-2}\right)$ many bulk squares $\alpha$, this bounds the sum over the terms $\alpha=\beta$ as claimed.

Next we consider the off-diagonal terms $\alpha \neq \beta$; we drop sub- and superscripts from $L$ and $\rho$ to shorten notations. We use a Taylor expansion to find that the sum of these terms is bounded, up to remainder terms, by

$$
\begin{aligned}
& N^{2} \sum_{\alpha \neq \beta} \iint_{\alpha \times \beta} \iint_{\alpha \times \beta}\left[\nabla \rho(\alpha)(z-\zeta)+\nabla^{2} \rho(\alpha)(z-\zeta)^{2}\right]\left[\nabla \rho(\beta)(w-\xi)+\nabla^{2} \rho(\beta)(w-\xi)^{2}\right] \\
\times & {\left[L(\alpha-\zeta)+\nabla L(\zeta-\xi)(z-\zeta-w+\xi)+\nabla^{2} L(\alpha-\xi)(z-\zeta-w+\xi)^{2}\right] m(\mathrm{~d} z) m(\mathrm{~d} w) m(\mathrm{~d} \zeta) m(\mathrm{~d} \xi) . }
\end{aligned}
$$

The remainder term are bounded similarly without using symmetry and produce the error terms depending on $\left\|\nabla^{3} \rho\right\|_{\infty}$. We return to the main terms. By symmetry, the odd terms in $(z-\xi)$ and $(w-\zeta)$ do not contribute. The leading terms are therefore the quartic terms. These terms are bounded by

$$
N^{2} b^{4}\left(\|\nabla \rho\|_{\infty}+\left\|\nabla^{2} \rho\right\|_{\infty}\right)^{2}
$$

The factor $b^{4}$ comes from $b^{-4} b^{4} b^{4}$ with the factor $b^{-4}$ coming from the summation over squares; the $b^{4}$ factor coming from the volume of the integration of $z$ and $w$, and the last $b^{4}$ factor comes from the size of products of $(z-\zeta)$ and $(w-\xi)$ in the formula. This concludes the proof. 
Proof of 7.25. We now bound $\mathbb{E}^{\Psi} \mathbb{E}^{\omega} \Omega_{\alpha \beta}$ for $\beta=B$ and $\alpha \subset D$. Since $\tilde{\mu}_{V}=\hat{\mu}-\mu_{V}$ and $\left.\mathbb{E}^{\Psi} \mathbb{E}^{\omega} \hat{\mu}\right|_{\alpha}$ is the uniform measure on $\alpha$ with total mass $\bar{n}_{\alpha} / N$, we have

$$
\begin{aligned}
\mathbb{E}^{\Psi} \mathbb{E}^{\omega}\left(\left.\tilde{\mu}_{V}\right|_{\alpha}(\mathrm{d} z)\right) & =\left.\left(\frac{\bar{n}_{\alpha}}{N b^{2} \rho_{V}(z)}-1\right) \mu_{V}\right|_{\alpha}(\mathrm{d} z), \\
\mathbb{E}^{\Psi} \mathbb{E}^{\boldsymbol{\omega}}\left(\left.\tilde{\mu}_{V}\right|_{B}(\mathrm{~d} z)\right) & =\left.\left(\frac{\bar{n}_{B}}{N \mu_{V}(B)}-1\right) \mu_{V}\right|_{B}(\mathrm{~d} z) .
\end{aligned}
$$

Since $\left.\hat{\mu}\right|_{B}$ and the $\left.\hat{\mu}\right|_{\alpha}$ are independent under $\mathbb{E}^{\Psi} \mathbb{E}^{\omega}$, and since the number of squares $\alpha$ is $\mathrm{O}\left(b^{-2}\right)$ and bounding $L_{R}^{\ell}$ by $\mathrm{O}(\log N)$, therefore

$$
\begin{aligned}
& N^{2} \sum_{\alpha} \mathbb{E}^{\Psi} \mathbb{E}^{\omega} \int_{z \in B} \int_{w \in \alpha} L_{R}^{\ell}\left(\Psi_{B}(z)-\Psi_{\alpha}(w)\right) \tilde{\mu}_{V}(\mathrm{~d} z) \tilde{\mu}_{V}(\mathrm{~d} w) \\
& =\left(\frac{\bar{n}_{B}}{\mu_{V}(B)}-N\right) \sum_{\alpha} \int_{z \in B} \int_{w \in \alpha} L_{R}^{\ell}(z-w)\left(\frac{\bar{n}_{\alpha}}{b^{2} \rho_{V}(z)}-N\right) \mu_{V}(\mathrm{~d} z) \mu_{V}(\mathrm{~d} w) \\
& =\mathrm{O}\left((\log N)\left|\bar{n}_{B}-N \mu_{V}(B)\right| \sum_{\alpha}\left|\bar{n}_{\alpha}-N \mu_{V}(\alpha)\right|\right)=\mathrm{O}\left((\log N) b^{-2}\right) .
\end{aligned}
$$

Similarly, for $\alpha=\beta=B$, we have

$$
\begin{aligned}
& N^{2} \mathbb{E}^{\Psi} \mathbb{E}^{\omega} \int_{z \in B} \int_{w \in B} L_{R}^{\ell}(z-w) \mathbb{1}_{z \neq w} \tilde{\mu}(\mathrm{d} z) \tilde{\mu}(\mathrm{d} w) \\
& =\left(\frac{\bar{n}_{B}}{\mu_{V}(B)}-N\right)^{2} \iint_{B \times B} L_{R}^{\ell}(z-w) \mu_{V}(\mathrm{~d} z) \mu_{V}(\mathrm{~d} w) \\
& =\left(\frac{\bar{n}_{B}}{\mu_{V}(B)}-N\right)^{2} \mathrm{O}(\log N) \mu_{V}(B)^{2}=\mathrm{O}\left(\bar{n}_{B} \log N\right) .
\end{aligned}
$$

This completes the proof.

7.4. Summary. In order to prove the lower bound for the partition function of the Coulomb gas, we used a quasi-local approximation whose main building blocks are Yukawa gases on torus. Due to the natural that the lower bound is proved via a variational trial state, all estimates needed are with respect to a Yukawa gas on tori. In particular, the rigidity estimate needed in the lower bound is with respect to a Yukawa gas on a torus. This rigidity estimate is done in Appendix A

\section{Proof of Theorem 1.2; central limit theorem}

In this section, we prove Theorem 1.2. Our proof uses a modification of the loop equation, which is a relation between one- and two-point correlations. It allows to obtain the moment generating function for linear statistics of the Coulomb plasma in terms of expectations of terms involving one-point and two-point correlations with respect to a tilted measure. The density estimates of Section 2 provide sufficient control on the one-point terms in the loop equation. The twopoint correlation term in the loop equation is singular and can be decomposed into short- and long-range contributions. The long-range part can be decomposed further into scales which can then be bounded using local density estimates for all scales. Thus the short-range contribution, which we call the local angle term, is the main difficulty to obtain the CLT.

In [5], the loop equation was used to prove a central limit theorem for $\beta=1$, by bounding the two-point contribution using the determinantal structure of the microscopic point process 
(which holds only for $\beta=1$ ). In [6], we used the loop equation approach for Coulomb plasma for any $\beta>0$ to obtain rigidity estimates, by introducing the local density estimates to estimate the long-range part of the two-point contribution and bounding the angle term by a trivial bound. In this section, we obtain an effective estimate for the angle term for general $\beta>0$. We deduce this estimate from Theorem 1.1 and the fact that the estimates for the remaining terms in the loop equation can be obtained also for version of the Coulomb plasma that is tilted by a small two-body interaction.

We remind the reader that all estimates in this section are with respect to a Coulomb gas with or without an angle correction term; the Yukawa gas is used only in the approximation of the free energy of the Coulomb gas, in Sections 4. 7. In particular, the estimate of the angle term, to be presented in this section, is with respect to a Coulomb gas. This estimate requires not just the local density bound, but the sophisticated rigidity estimate which is a consequence of the loop equation. The rigidity estimate will also be needed for Yukawa gases on a torus, to be presented in the Appendix A.

8.1. CLT for macroscopic test functions. We first prove Theorem 1.2 for macroscopic test functions $f$. For this, we first prove that a version of Theorem 1.2 holds up to certain random shift, the local angle term $\hat{A}_{V}^{f}$ defined by

$$
\hat{A}_{V}^{f}=\frac{N}{2} \operatorname{Re} \iint_{z \neq w} \frac{h(z)-h(w)}{z-w} \mathrm{e}^{-\frac{|z-w|^{2}}{2 \theta^{2}}} \tilde{\mu}_{V}(\mathrm{~d} z) \tilde{\mu}_{V}(\mathrm{~d} w), \quad h(z)=\frac{\bar{\partial} f(z)}{\partial \bar{\partial} V(z)},
$$

where $\theta=N^{-1 / 2+\sigma}$. Note the integrand is singular at $z=w$ since

$$
\frac{h(z)-h(w)}{z-w}=\partial h(z)+\bar{\partial} h(z) \frac{\bar{z}-\bar{w}}{z-w}+\mathrm{O}(|z-w|) .
$$

We recall the definitions of $X_{V}^{f}$ and $Y_{V}^{f}$ from 1.11) and (1.12), as well as the norms from (1.7), and that we write $\|f\|_{\infty, k}$ for $\|f\|_{\infty, k, b}$ with $b=1$.

In the proof of [6, Theorem 1.2], more precisely in [6, Lemma 7.5], we showed that (8.1) is bounded by $\mathrm{O}\left(N^{\varepsilon}\right)$ with very high probability. Assuming this term was $\ll 1$ instead of $\mathrm{O}\left(N^{\varepsilon}\right)$, a small modification of the argument in [6, Section 7] would already imply Theorem 1.2. A similar strategy was used in [4,5], where a version of (8.1) was shown to be approximately equal to $-\frac{1}{2} Y_{V}^{f}$ for $\beta=1$, by using the exactly known correlation kernel for the microscopic correlation functions in this integrable case. Our strategy now is to first prove a version of Theorem 1.2 in which the contribution of the angle term (8.1) has been removed (in Proposition 8.1 below), and then subsequently, by combining this argument with Theorem 1.1. prove that the angle term 8.1) is in fact negligible up to the constant $-\frac{1}{2} Y_{V}^{f}$ (in Proposition 8.2 .

Proposition 8.1. Suppose that $V$ satisfies conditions $(1.4)$ and 1.10 , or more generally the conditions stated in Remark 4.2. Then for any small $\sigma$, the following holds. For any function $f$ satisfying the same assumptions as in Theorem 1.2 (in particular the support of $f$ has distance of order 1 to $\left.\partial S_{V}\right)$, for small $\varepsilon$ and $t b^{-2} N^{2 \sigma}+t b^{-2}\|f\|_{\infty, 4, b} \ll 1$, we have for any $0 \leq|u| \leq \mathrm{O}(t)$

$$
\begin{aligned}
& \quad \frac{1}{t \beta N} \log \mathbb{E}_{V} \mathrm{e}^{-\beta N t\left(X_{V}^{f}-\hat{A}_{V+u f}^{f}\right)}=\frac{t N}{8 \pi} \int|\nabla f(z)|^{2} m(\mathrm{~d} z)-\frac{1}{\beta} Y_{V}^{f} \\
& +\mathrm{O}\left(N^{-1 / 2+3 \sigma+\varepsilon} b^{-1}+N^{-\sigma+\varepsilon}\right)\|f\|_{\infty, 3, b}+\mathrm{O}\left(t N^{2 \sigma+\varepsilon} b^{-2}\right)\|f\|_{\infty, 3, b}^{2}+\mathrm{O}\left(N^{-1 / 2+\varepsilon} b\|f\|_{4, b}\right) .
\end{aligned}
$$

Proposition 8.2. There exists $\kappa>0$ such that if $\sigma=\kappa / 6$ and $0 \leq|u|, t \leqslant N^{-2 \kappa / 3}$,

$$
\frac{1}{t \beta N} \log \mathbb{E}_{V} \mathrm{e}^{\beta N t \hat{A}_{V+u f}^{f}}=-\frac{1}{2} Y_{V}^{f}+\mathrm{O}\left(N^{-\kappa / 3}\right)\left(1+\|f\|_{\infty, 5}\right)^{2} .
$$


The above two propositions will be proved in Sections 8.28 .5 below. We first note that the estimate 8.2 given by Proposition 8.1 without the angle term $\hat{A}_{V+u f}^{f}$ on the left-hand side would imply a CLT for $X_{V}^{f}$. This angle term is controlled by $(8.3)$ of Proposition 8.2 . By combining the two estimates, we can complete the proof of Theorem 1.2 for macroscopic test functions. For mesoscopic test functions, a similar argument applies after conditioning (see Section 8.6).

Proof of Theorem 1.2 for macroscopic test functions. By assumption, $f$ is a macroscopic test function with $\|f\|_{\infty, 5}$ bounded. Let $\sigma$ and $\kappa$ be as in Proposition 8.2. Then, with $\lambda=N t$ in the identity

$$
\frac{1}{t \beta N} \log \mathbb{E}_{V} \mathrm{e}^{-\beta N t X_{V}^{f}}=\frac{1}{t \beta N}\left(\log \mathbb{E}_{V} \mathrm{e}^{-\beta N t\left(X_{V}^{f}-\hat{A}_{V}^{f}\right)}-\log \mathbb{E}_{V+t f} \mathrm{e}^{\beta N t \hat{A}_{V}^{f}}\right),
$$

the claim follows by using the estimates $(8.2),(8.3)$ for the two terms on the right-hand side of (8.4), and finally replacing $\kappa$ by $3 \kappa$.

8.2. Loop equation with angle term. We start the proof with an integration by parts formula. Consider a smooth bounded function $v: \mathbb{C} \rightarrow \mathbb{C}$, and $G$ smooth, defined on $z_{1} \neq z_{2}$ such that $G\left(z_{1}, z_{2}\right)=G\left(z_{2}, z_{1}\right)$, and

$$
\limsup _{\left|z_{2}\right| \rightarrow \infty}\left(\left|G\left(z_{1}, z_{2}\right)\right| / \log \left|z_{2}\right|\right) \leqslant 1
$$

for any fixed $z_{1}$. For any $\mathbf{z} \in \mathbb{C}^{N}$ we define

$$
W_{V}^{G, v}(\mathbf{z})=-\sum_{j \neq k}\left(v\left(z_{j}\right)-v\left(z_{k}\right)\right) \partial_{z_{j}} G\left(z_{j}, z_{k}\right)+\frac{1}{\beta} \sum_{j} \partial_{j} v\left(z_{j}\right)-N \sum_{j} v\left(z_{j}\right) \partial V\left(z_{j}\right) .
$$

The following elementary lemma is often referred to as Ward identity or loop equation. For example, it was used in [5] to study fluctuations of the empirical measure when $\beta=1$, and in 6 to prove rigidity for all $\beta>0$, with in both cases the interaction $G$ being the Coulomb potential $\mathcal{C}$. Its relation to Conformal Field Theory is discussed in [29]. In this work we need a perturbation $G$ of the Coulomb interaction by the local angle term.

Lemma 8.3. Under the above assumptions, we have

$$
\mathbb{E}_{V}^{G}\left(W_{V}^{G, v}\right)=\frac{1}{2} \mathbb{E}_{V}^{G}\left(\sum_{j \neq k}\left(v\left(z_{j}\right)+v\left(z_{k}\right)\right)\left(\partial_{z_{k}}+\partial_{z_{j}}\right) G\left(z_{j}, z_{k}\right)\right),
$$

where the expectation is with respect to $P_{N, V}^{G}$ defined in 1.3 .

Proof. The proof is a classical simple integration by parts: for any $j \in \llbracket 1, N \rrbracket$, we have

$$
\mathbb{E}\left(\partial_{z_{j}} v\left(z_{j}\right)\right)=\beta \mathbb{E}\left(v\left(z_{j}\right) \partial_{z_{j}} H(\mathbf{z})\right),
$$

where both terms are absolutely summable and the boundary terms vanishes because (i) with probability 1 , no two $z_{i}$ 's have the same real or imaginary part, (ii) $v$ is bounded, $G$ satisfies the growth condition (8.5), $V$ satisfies the growth condition (1.4). Summation of the above equation over all $j \in \llbracket 1, N \rrbracket$ therefore gives

$$
\begin{aligned}
& \frac{1}{\beta N} \sum_{j=1}^{N} \mathbb{E}\left(\partial_{z_{j}} v\left(z_{j}\right)\right)=\mathbb{E}\left(\sum_{j=1}^{N} v\left(z_{j}\right)\left(\partial_{z_{j}} V\left(z_{j}\right)+\sum_{k \neq j}\left(\partial z_{j} G\left(z_{j}, z_{k}\right)+\partial z_{j} G\left(z_{k}, z_{j}\right)\right)\right)\right) \\
& =\mathbb{E}\left(\sum_{j=1}^{N} v\left(z_{j}\right)\left(\partial_{z_{j}} V\left(z_{j}\right)+\sum_{k \neq j}\left(\partial z_{j}-\partial z_{k}\right) G\left(z_{j}, z_{k}\right)\right)\right)+\mathbb{E}\left(\sum_{j=1} \sum_{k \neq j} v\left(z_{j}\right)\left(\partial z_{j}+\partial z_{k}\right) G\left(z_{j}, z_{k}\right)\right) .
\end{aligned}
$$


Using $G\left(z_{j}, z_{k}\right)=G\left(z_{k}, z_{j}\right)$, we can continue the equation with

$$
\begin{aligned}
& =\mathbb{E}\left(\sum_{j=1}^{N} v\left(z_{j}\right) \partial_{z_{j}} V\left(z_{j}\right)+\frac{1}{2} \sum_{j \neq k}\left(v\left(z_{j}\right)\left(\partial z_{j}-\partial z_{k}\right) G\left(z_{j}, z_{k}\right)+v\left(z_{k}\right)\left(\partial z_{k}-\partial z_{j}\right) G\left(z_{k}, z_{j}\right)\right)\right) \\
& \quad+\frac{1}{2} \mathbb{E}\left(\sum_{j \neq k}\left(v\left(z_{j}\right)+v\left(z_{k}\right)\right)\left(\partial z_{j}+\partial z_{k}\right) G\left(z_{j}, z_{k}\right)\right) \\
& =\mathbb{E}\left(\sum_{j=1}^{N} v\left(z_{j}\right) \partial_{z_{j}} V\left(z_{j}\right)+\frac{1}{2} \sum_{j \neq k}\left(v\left(z_{j}\right)-v\left(z_{k}\right)\right)\left(\partial z_{j}-\partial z_{k}\right) G\left(z_{j}, z_{k}\right)\right) \\
& \quad+\frac{1}{2} \mathbb{E}\left(\sum_{j \neq k}\left(v\left(z_{j}\right)+v\left(z_{k}\right)\right)\left(\partial z_{j}+\partial z_{k}\right) G\left(z_{j}, z_{k}\right)\right) .
\end{aligned}
$$

This concludes the proof.

Before considering the interaction $G$ with additional angle term, we temporarily restrict our attention to the Coulomb case, where $\partial_{z_{j}} \mathcal{C}\left(z_{j}-z_{k}\right)=-\frac{1}{2}\left(z_{j}-z_{k}\right)^{-1}$.

Lemma 8.4. For any $f: \mathbb{C} \rightarrow \mathbb{R}$ of class $\mathscr{C}^{2}$ supported on $S_{V}$ and $\mathbf{z} \in \mathbb{C}^{N}$, recall $X_{V}^{f}$ defined in (1.11) and $h$, depending on $f$ and $V$, defined in (8.1). With these notations, we have

$$
X_{V}^{f}=-\frac{1}{N} W_{V}^{h}(\mathbf{z})+\frac{1}{N \beta} \sum_{k} \partial h\left(z_{k}\right)+\frac{N}{2} \iint_{z \neq w} \frac{h(z)-h(w)}{z-w} \tilde{\mu}_{V}(\mathrm{~d} z) \tilde{\mu}_{V}(\mathrm{~d} w),
$$

where $\tilde{\mu}_{V}=\hat{\mu}-\mu_{V}$ and we used the notation $W_{V}^{h}=W_{V}^{\mathcal{C}, h}$.

Proof. First remember the following two identities:

$$
\int \frac{\mu_{V}(\mathrm{~d} w)}{z-w}=\partial V(z), \quad f(z)=\frac{1}{\pi} \int \frac{\bar{\partial} f(w)}{z-w} m(\mathrm{~d} w)
$$

The first equation holds for $z \in S_{V}$ and is obtained by the Euler-Lagrange equation, the second equation is a simple integration by parts. We therefore can write

$$
\begin{aligned}
X_{V}^{f} & =\sum_{j} \int \frac{h(w)}{z_{j}-w} \mu_{V}(\mathrm{~d} w)-N \iint \frac{h(w)}{z-w} \mu_{V}(\mathrm{~d} w) \mu_{V}(\mathrm{~d} z) \\
& =N \iint \frac{h(w)-h(z)}{z-w} \hat{\mu}_{V}(\mathrm{~d} z) \mu_{V}(\mathrm{~d} w)+\sum_{j} h\left(z_{j}\right) \partial V\left(z_{j}\right)-\frac{N}{2} \iint \frac{h(w)-h(z)}{z-w} \mu_{V}(\mathrm{~d} w) \mu_{V}(\mathrm{~d} z) \\
& =-\frac{1}{2 N} \sum_{j \neq k} \frac{h\left(z_{j}\right)-h\left(z_{k}\right)}{z_{j}-z_{k}}+\sum_{j} h\left(z_{j}\right) \partial V\left(z_{j}\right)+\frac{N}{2} \iint_{z \neq w} \frac{h(z)-h(w)}{z-w} \tilde{\mu}_{V}(\mathrm{~d} z) \tilde{\mu}_{V}(\mathrm{~d} w),
\end{aligned}
$$

which is equivalent to 8.7 . In the first equation we used (1.6) and (8.8), and in the second equation we used 8.8 .

We now decompose the last term in 8.7 into a sum of the long-range and short-range terms. 
For this purpose, let $\varphi(z)=\mathrm{e}^{-|z|^{2}}$ and, given a mesoscopic scale $\theta=N^{-\frac{1}{2}+\sigma}$, we define

$$
\begin{aligned}
\Phi(z-w, r) & =\frac{2}{\pi} \int \varphi\left(\frac{|z-\xi|}{r}\right) \varphi\left(\frac{|\xi-w|}{r}\right) \mathrm{d} m(\xi)=r^{2} \mathrm{e}^{-\frac{|z-w|^{2}}{2 r^{2}}}, \\
\Phi_{\theta}^{-}(z-w) & =\int_{0}^{\theta} \Phi(z-w, r) \frac{\mathrm{d} r}{r^{5}}=\frac{\mathrm{e}^{-\frac{|z-w|^{2}}{2 \theta^{2}}}}{|z-w|^{2}}, \\
\Phi_{\theta}^{+}(z-w) & =\int_{\theta}^{\infty} \Phi(z-w, r) \frac{\mathrm{d} r}{r^{5}}=\frac{1-\mathrm{e}^{-\frac{|z-w|^{2}}{2 \theta^{2}}}}{|z-w|^{2}}, \\
\Psi_{h}^{ \pm}(z, w) & =\Phi_{\theta}^{ \pm}(z-w)(\bar{z}-\bar{w})(h(z)-h(w)), \quad \Psi^{h}(z, w)=\Psi_{h}^{+}(z, w)+\Psi_{h}^{-}(z, w) .
\end{aligned}
$$

As in the proof of [6, Lemma 7.5] (see also [20]), we have decomposed the last term in (8.7) into a relatively long range part and, essentially, a local angle term:

$$
\frac{N}{2} \iint_{z \neq w} \frac{h(z)-h(w)}{z-w} \tilde{\mu}_{V}(\mathrm{~d} z) \tilde{\mu}_{V}(\mathrm{~d} w)=A_{V}^{h,+}+A_{V}^{h,-},
$$

where

$$
\begin{aligned}
& A_{V}^{h,+}=\frac{N}{2} \iint_{z \neq w} \Psi_{h}^{+}(z, w) \tilde{\mu}_{V}(\mathrm{~d} z) \tilde{\mu}_{V}(\mathrm{~d} w), \\
& A_{V}^{h,-}=\frac{N}{2} \iint_{z \neq w} \Psi_{h}^{-}(z, w) \tilde{\mu}_{V}(\mathrm{~d} z) \tilde{\mu}_{V}(\mathrm{~d} w) .
\end{aligned}
$$

By definition 8.1), we also have

$$
\hat{A}_{V}^{f}=\frac{N}{2} \operatorname{Re} \iint_{z \neq w} \frac{h(z)-h(w)}{z-w} \mathrm{e}^{-\frac{|z-w|^{2}}{2 \theta^{2}}} \tilde{\mu}_{V}(\mathrm{~d} z) \tilde{\mu}_{V}(\mathrm{~d} w)=\operatorname{Re} A_{V}^{h,-},
$$

i.e., $\hat{A}_{V}^{f}$ is just $\operatorname{Re} A_{V}^{h,-}$ with $h$ chosen according to 8.1).

Note that, in the above decomposition, we could have considered any fixed non-negative function $\varphi \in C^{\infty}(\mathbb{C})$ with compact support or fast decay at infinity, as in [6, Lemma 7.5]. We here chose the Gaussian scale function for the sake of concreteness and some convenient simplifications. Compared with $[6]$, we also write the mesoscopic scale as $\theta$ rather than $N^{-1 / 2} \theta$.

8.3. Coulomb gas with angle perturbation. We now define the perturbed Coulomb gas. The Coulomb gas, exponentially tilted by the real-part of the local angle term, is defined to have pair interaction and potential given by

$$
G_{t}=\mathcal{C}-\frac{t}{2} \operatorname{Re} \Psi_{h}^{-}, \quad V_{t}=V+t f+t F, \quad F=\operatorname{Re} \int \Psi_{h}^{-}(\cdot, w) \mu_{V}(\mathrm{~d} w),
$$

where $h=\frac{\bar{\partial} f}{\partial \bar{\partial} V}$ (we will see that $h=h_{0}$ defined in 8.17 below). We also include a $t$-dependent constant in the perturbed Hamiltonian and define

$$
H_{t}:=H_{V_{t}}^{G_{t}}-\frac{t}{2} N^{2} \operatorname{Re} \iint \Psi_{h}^{-}(z, w) \mu_{V}(\mathrm{~d} z) \mu_{V}(\mathrm{~d} w)=H_{V+t f}^{\mathcal{C}}-N t \hat{A}_{V}^{f} .
$$

Notice that the interaction term involving $\operatorname{Re} \Psi_{h}^{-}$, the potential term involving $t F$ and the constant term in 8.15) were recombined into $\hat{A}_{V}^{f}$ which was defined in 8.1). Notice further that the subscript $V$ is different from the subscript $V+t f$ in the Hamiltonian in 8.15).

For the proof of Proposition 8.1, we require the following Proposition 8.5 regarding a local density estimate for this interaction. 
Proposition 8.5. Consider the Coulomb gas with Hamiltonian (8.15), with $V, f \in \mathscr{C}^{2}$ and $t N^{2 \sigma} \leqslant$ 1 and $\|\nabla h\|_{\infty} \leqslant 1$ and $t \in[0,1]$. For all $s \in\left(0, \frac{1}{2}\right)$, for all $f$ supported in ball of radius $b=N^{-s}$ contained in $S_{V}$ with distance of order 1 to the boundary, we have the local density estimate

$$
X_{f}^{V_{t}} \prec \sqrt{N b^{2}}\|f\|_{\infty, 2, b}
$$

with respect to the measure $P_{V_{t}}^{G_{t}}$. In particular, for any ball as above, the number of particles in that ball is bounded by $\mathrm{O}\left(N b^{2}\right)$ with high probability.

Proof. The proposition is a direct consequence of Theorem 2.3. Indeed, 8.15 corresponds to the choice $\tilde{G}(z, w)=\frac{h(z)-h(w)}{z-w} \mathrm{e}^{-|z-w|^{2} /\left(2 \theta^{2}\right)}$ in 2.8 which satisfies 2.9 since $\|\nabla h\|_{\infty} \leqslant 1$.

For $0 \leq t \ll 1$, we define

$$
h_{t}(z)=\frac{\bar{\partial} f(z)}{\partial \bar{\partial}(V(z)+t f(z))}, \quad h=h_{0} .
$$

In the next lemma, we collect some elementary estimates for $h_{t}$ and $F_{t}$. Recall that $V$ satisfies the growth condition (1.4) and the regularity assumption 1.10 .

Lemma 8.6. Assume that the support of $f$ has distance $\gg N^{-1 / 2+\sigma}$ to $\partial S_{V}$, and that

$$
t b^{-2}\|f\|_{\infty, 4, b} \ll 1 .
$$

Recall $\theta=N^{-1 / 2+\sigma}$. Then the following estimates hold:

$$
\begin{aligned}
\left\|h_{t}\right\|_{k, b} & \leq b^{-1}\|f\|_{\infty, k+1, b}\left[1+t b^{-2}\|f\|_{\infty, k+2, b}\right] \\
t F(z) & =\mathrm{O}\left(N^{-1+2 \sigma}\right) t b^{-2}\|f\|_{\infty, 2, b} \\
t \Delta F(z) & =\mathrm{O}\left(N^{-1 / 2+\sigma}\right) t b^{-3}\|f\|_{\infty, 4, b}
\end{aligned}
$$

Proof. Using that $t\|\Delta f\|_{\infty} \ll 1$ and (8.18), we have

$$
\left\|\nabla h_{t}\right\|_{\infty} \leq \frac{\|\nabla \bar{\partial} f\|_{\infty}}{\|\partial \bar{\partial}(V+t f)\|_{\infty}}+\frac{\|\bar{\partial} f \nabla(\partial \bar{\partial}(V+t f))\|_{\infty}}{\|\partial \bar{\partial}(V+t f)\|_{\infty}^{2}} \leq b^{-2}\|f\|_{\infty, 2, b}\left[1+t b^{-2}\|f\|_{\infty, 3, b}\right] .
$$

Similar estimates hold for higher derivatives and we get in general (8.19). We can bound $t F$ by

$$
t F(z)=t \int \frac{h(z)-h(w)}{z-w} \mathrm{e}^{-\frac{|z-w|^{2}}{2 \theta^{2}}} \mu_{V}(\mathrm{~d} w)=\mathrm{O}\left(N^{-1+2 \sigma}\right) t\|\nabla h\|_{\infty}=\mathrm{O}\left(N^{-1+2 \sigma}\right) t b^{-2}\|f\|_{\infty, 2, b},
$$

which is a small correction to $V+t f$. Similarly, we have

$$
\begin{gathered}
t \Delta F(z)=t \Delta h(z) \int \frac{\mathrm{e}^{-\frac{|z-w|^{2}}{2 \theta^{2}}}}{z-w} \mu_{V}(\mathrm{~d} w)-2 t \nabla h(z) \int\left(\nabla_{w} \frac{\mathrm{e}^{-\frac{|z-w|^{2}}{2 \theta^{2}}}}{z-w}\right) \mu_{V}(\mathrm{~d} w) \\
+t h(z) \int\left(\Delta_{w} \frac{\mathrm{e}^{-\frac{|z-w|^{2}}{2 \theta^{2}}}}{z-w}\right) \mu_{V}(\mathrm{~d} w)=\mathrm{O}\left(N^{-1 / 2+\sigma+\varepsilon}\right),
\end{gathered}
$$

where for the last estimate we integrated $w$ by parts to avoid the singularity.

By using the local law of Proposition 8.5 in the loop equation, as in [6, Section 7], we obtain the following estimate. Recall that $\hat{A}_{V}^{f}$ was defined in 8.1 and satisfies 8.13 . 
Lemma 8.7. Suppose that the assumption (8.18) holds. Recall $\sigma$ is the parameter in the definition (8.1). Then for any $0 \leq|u| \leq \mathrm{O}(t)$

$$
\begin{aligned}
\frac{1}{t \beta N} \log \mathbb{E}_{V} \mathrm{e}^{-t \beta N\left(X_{V}^{f}-\hat{A}_{V+u f}^{f}\right)}=\frac{t N}{8 \pi} \int|\nabla f(z)|^{2} m(\mathrm{~d} z)-\frac{1}{\beta} Y_{V}^{f}+\frac{1}{t} \operatorname{Re} \int_{0}^{t} \mathbb{E}_{V_{s}}^{G_{s}}\left(A_{V_{s}}^{h_{s},+}\right) \mathrm{d} s \\
+\mathrm{O}\left(N^{-1 / 2+3 \sigma+\varepsilon} b^{-1}\|f\|_{3, b}\right)+\mathrm{O}\left(t N^{\varepsilon} b^{-2} N^{2 \sigma}\|f\|_{3, b}^{2}\right)+\mathrm{O}\left(N^{-1 / 2+\varepsilon} b\|f\|_{4, b}\right), \quad(8.22) \\
\frac{1}{t \beta N} \log \mathbb{E}_{V} \mathrm{e}^{-t \beta N X_{V}^{f}}=\frac{t N}{8 \pi} \int|\nabla f(z)|^{2} m(\mathrm{~d} z)-\frac{1}{\beta} Y_{V}^{f}+\frac{1}{t} \operatorname{Re} \int_{0}^{t} \mathbb{E}_{V+s f}^{\mathcal{C}}\left(A_{V+s f}^{h_{s},-}+A_{V+s f}^{h_{s},+}\right) \mathrm{d} s \\
+\mathrm{O}\left(N^{-1 / 2+3 \sigma+\varepsilon} b^{-1}\|f\|_{3, b}\right)+\mathrm{O}\left(t N^{\varepsilon} b^{-2} N^{2 \sigma}\|f\|_{3, b}^{2}\right)+\mathrm{O}\left(N^{-1 / 2+\varepsilon} b\|f\|_{4, b}\right) .
\end{aligned}
$$

Proof. We focus on 8.22 ; the second bound 8.23 can be proved in a similar way. Note that the expectation on the right-hand side of 8.23 is with respect to the standard Coulomb gas without local angle term, and that the terms $A_{V+s f}^{h_{s}, \pm}$ are with respect to the external potential $V+s f$. The estimate (8.23) was essentially obtained in [6, Section 7] already. The short range angle term, $A_{V+s f}^{h_{s},-}$, was difficult to estimate in $[6$. Section 7]. In 8.22 , we added an angle term in the Hamiltonian so that there is no such short range angle term on the right side of 8.22 . The following proof is written for $u=0$ for the simplicity of notations; we will remark on the modification needed for the general case in the proof. Furthermore, the error $A_{V_{t}}^{h_{t},-}-A_{V+u f}^{h,-}$ will be estimated in Lemma 8.8.

We denote by $Z_{t}$ the partition function corresponding to the Hamiltonian (8.15). Then the left-hand side of 8.22 can be written as

$$
\frac{1}{t \beta N}\left(\log Z_{t}-\log Z_{0}\right)+N \int f \mathrm{~d} \mu_{V}=\frac{1}{t} \int_{0}^{t}\left[\partial_{s} \frac{1}{\beta N} \log Z_{s}+N \int f \mathrm{~d} \mu_{V}\right] \mathrm{d} s .
$$

Using the definition 8.15 of $G_{t}$, we get

$$
\partial_{t} \frac{1}{\beta N} \log Z_{t}+N \int f \mathrm{~d} \mu_{V}=N \int f\left(\mathrm{~d} \mu_{V}-\mathrm{d} \mu_{V_{t}}\right)+\operatorname{Re} \mathbb{E}_{V_{t}}^{G_{t}}\left(-X_{V_{t}}^{f}+\hat{A}_{V}^{f}\right) .
$$

As $t \ll 1$ and $\Delta f$ is bounded and supported in $S_{V}$, the supports $S_{V}$ and $S_{V_{t}}$ coincide. Together with the explicit formula for the equilibrium measure (1.6) and with (8.20), we have

$$
\begin{aligned}
N \int f\left(\mathrm{~d} \mu_{V}-\mathrm{d} \mu_{V_{t}}\right) & =\frac{N t}{4 \pi} \int|\nabla f|^{2} \mathrm{~d} m+\frac{N}{4 \pi} \int|f t \Delta F| \mathrm{d} m \\
& =\frac{N t}{4 \pi} \int|\nabla f|^{2} \mathrm{~d} m+\mathrm{O}\left(t b^{-2} N^{2 \sigma}\right)\|f\|_{\infty, 2, b}^{2},
\end{aligned}
$$

where we have integrated by parts twice in getting the last inequality and also used that the support of the integrand has area $\mathrm{O}\left(b^{2}\right)$. Using (8.7) with the choice $V_{t}$ for the external potential (and the unperturbed Coulomb pair interaction), we have

$$
\mathbb{E}_{V_{t}}^{G_{t}}\left(-X_{V_{t}}^{f}+A_{V}^{h,-}\right)=\mathbb{E}_{V_{t}}^{G_{t}}\left(\frac{1}{N} W_{V_{t}}^{h_{t}}-\frac{1}{N \beta} \sum_{k} \partial h_{t}\left(z_{k}\right)-A_{V_{t}}^{h_{t},+}-A_{V_{t}}^{h_{t},-}+A_{V}^{h,-}\right) .
$$

The perturbed interaction $G_{t}$ satisfies $G_{t}\left(z_{j}, z_{k}\right)=G_{t}\left(z_{k}, z_{j}\right)$ and the growth assumption (8.5), so Lemma 8.3 applies. Together with the definition of $G_{t}$ and recalling $W_{V}^{h}=W_{V}^{\mathcal{C}, h}$, we have

$$
\begin{aligned}
\mathbb{E}_{V_{t}}^{G_{t}}\left(W_{V_{t}}^{h_{t}}+t \sum_{j \neq k}\left(h_{t}\left(z_{j}\right)-h_{t}\left(z_{k}\right)\right) \partial_{z_{j}} \operatorname{Re} \Psi_{h_{t}}^{-}\left(z_{j}, z_{k}\right)\right)=\mathbb{E}_{V_{t}}^{G_{t}}\left(W_{V_{t}}^{G_{t}, h_{t}}\right) \\
=\frac{1}{2} \mathbb{E}_{V_{t}}^{G_{t}}\left(\sum_{j \neq k}\left(h_{t}\left(z_{j}\right)+h_{t}\left(z_{k}\right)\right)\left(\partial_{z_{k}}+\partial_{z_{j}}\right) G_{t}\left(z_{j}, z_{k}\right)\right) .
\end{aligned}
$$


In summary, equations 8.24 and 8.25 give

$$
\begin{array}{r}
\partial_{t} \frac{1}{\beta N} \log Z_{t}+N \int f \mathrm{~d} \mu_{V}=\frac{N t}{4 \pi} \int|\nabla f|^{2} \mathrm{~d} m+\operatorname{Re} \mathbb{E}_{V_{t}}^{G_{t}}\left(-\frac{1}{N \beta} \sum_{k} \partial h_{t}\left(z_{k}\right)-A_{V_{t}}^{h_{t},+}-A_{V_{t}}^{h_{t},-}+A_{V}^{h,-}\right. \\
\left.-\frac{t}{N} \sum_{j \neq k}\left(h_{t}\left(z_{j}\right)-h_{t}\left(z_{k}\right)\right) \partial_{z_{j}} \operatorname{Re} \Psi_{h_{t}}^{-}\left(z_{j}, z_{k}\right)+\frac{1}{2 N} \sum_{j \neq k}\left(h_{t}\left(z_{j}\right)+h_{t}\left(z_{k}\right)\right)\left(\partial_{z_{k}}+\partial_{z_{j}}\right) G_{t}\left(z_{j}, z_{k}\right)\right) \\
+\mathrm{O}\left(t b^{-2} N^{2 \sigma}\right)\|f\|_{\infty, 2, b}^{2} . \quad(8.26)
\end{array}
$$

We now evaluate all terms in the above expectation. The difference $A_{V_{t}}^{h_{t},-}-A_{V}^{h,-}$ is bounded in Lemma 8.8 below. For the general cases with $u \neq 0, A_{V}^{h,-}$ should be replaced by $A_{V+u f}^{h,-}$. Notice that Lemma 8.8 is valid for all $0 \leq|u| \leq \mathrm{O}(t)$.

The other terms are bounded as follows. By 8.16,

$$
\operatorname{Re} \mathbb{E}_{V_{t}}^{G_{t}}\left(-\frac{1}{N \beta} \sum_{k} \partial h_{t}\left(z_{k}\right)\right)=-\frac{1}{\beta} \operatorname{Re} \int \partial h_{t} \mathrm{~d} \mu_{V_{t}}+\mathrm{O}\left(N^{-1 / 2+\varepsilon} b\right)\left\|\nabla h_{t}\right\|_{\infty, 2, b}
$$

To compute the main term on the right-hand side, recall that $V_{t}=(V+t f)+t F$. By integration by parts and the explicit formula for the equilibrium density,

$$
\begin{aligned}
-\frac{1}{\beta} \operatorname{Re} \int \partial h_{t} \mathrm{~d} \mu_{V_{t}} & =-\frac{1}{4 \pi \beta} \operatorname{Re} \int \partial\left(\frac{\bar{\partial} f}{\partial \bar{\partial}(V+t f)}\right) \Delta(V+t f) \mathrm{d} m+\mathrm{O}\left(t \int \partial h_{t} \Delta F \mathrm{~d} m\right) \\
& =-\frac{1}{4 \pi \beta} \int \Delta f \log \Delta(V+t f) \mathrm{d} m+\mathrm{O}\left(t \int \partial h_{t} \Delta F \mathrm{~d} m\right) \\
& =-\frac{1}{\beta} Y_{V}^{f}+\mathrm{O}\left(t \int|\Delta f|^{2} \mathrm{~d} m\right)+\mathrm{O}\left(t b^{-2} N^{2 \sigma}\right)\|f\|_{\infty, 3, b}^{2} \\
& =-\frac{1}{\beta} Y_{V}^{f}+\mathrm{O}\left(t b^{-2} N^{2 \sigma}\right)\|f\|_{\infty, 3, b}^{2}
\end{aligned}
$$

Finally, differentiating $\Psi$ and using 8.9 give

$$
\left|\frac{t}{N} \sum_{j \neq k}\left(h_{t}\left(z_{j}\right)-h_{t}\left(z_{k}\right)\right) \partial_{z_{j}} \operatorname{Re} \Psi_{h_{t}}^{-}\left(z_{j}, z_{k}\right)\right| \leqslant C \frac{t}{N}\left\|\nabla h_{t}\right\|_{\infty}^{2} \sum_{j \neq k: z_{j} \in \Omega} \mathrm{e}^{-\frac{\left|z_{j}-z_{k}\right|^{2}}{2 \theta^{2}}}\left(1+\frac{\left|z_{j}-z_{k}\right|^{2}}{\theta^{2}}\right)+\mathrm{e}^{-N^{\varepsilon}},
$$

where $\Omega$ is the $N^{\varepsilon} \theta$-neighborhood of the support of $h$. Using the boundedness of the local density, implied by (8.16), we have, under the assumption (8.18), that

$$
\begin{aligned}
\operatorname{Re} \mathbb{E}_{V_{t}}^{G_{t}}\left(\frac{t}{N} \sum_{j \neq k}\left(h_{t}\left(z_{j}\right)-h_{t}\left(z_{k}\right)\right) \partial_{z_{j}} \operatorname{Re} \Psi_{h_{t}}^{-}\left(z_{j}, z_{k}\right)\right) & =\mathrm{O}\left(t N^{2 \sigma+\varepsilon} b^{2}\right)\left\|\nabla h_{t}\right\|_{\infty}^{2} \\
& =\mathrm{O}\left(t b^{-2} N^{2 \sigma+\varepsilon}\right)\|f\|_{\infty, 2, b}^{2}
\end{aligned}
$$


Similarly, 8.9 yields

$$
\begin{aligned}
& \frac{1}{N} \sum_{j \neq k}\left(h_{t}\left(z_{j}\right)+h_{t}\left(z_{k}\right)\right)\left(\partial_{z_{j}}+\partial_{z_{k}}\right) G_{t}\left(z_{j}, z_{k}\right) \\
& =\frac{t}{N} \sum_{j \neq k}\left(h_{t}\left(z_{j}\right)+h_{t}\left(z_{k}\right)\right) \frac{\partial h\left(z_{j}\right)-\partial h\left(z_{k}\right)}{z_{j}-z_{j}} \mathrm{e}^{-\frac{\left|z_{k}-z_{j}\right|^{2}}{2 \theta^{2}}} \\
& =\mathrm{O}\left(\frac{t}{N}\left\|h_{t}\right\|_{\infty}\left\|\nabla^{2} h\right\|_{\infty} \sum_{j \neq k: z_{j} \in \Omega} \mathrm{e}^{-\frac{\left|z_{j}-z_{k}\right|^{2}}{2 \theta^{2}}}\right)+\mathrm{O}\left(\mathrm{e}^{-N^{\varepsilon}}\right) \\
& =\mathrm{O}\left(\frac{t}{N} b^{-4}\|f\|_{\infty, 3, b}^{2} \sum_{j \neq k: z_{j} \in \Omega} \mathrm{e}^{-\frac{\left|z_{j}-z_{k}\right|^{2}}{2 \theta^{2}}}\right)+\mathrm{O}\left(\mathrm{e}^{-N^{\varepsilon}}\right) .
\end{aligned}
$$

The local density estimate 8.16 then again gives

$$
\begin{aligned}
\operatorname{Re} \mathbb{E}_{V_{t}}^{G_{t}}\left(\frac{1}{N} \sum_{j \neq k}\left(h_{t}\left(z_{j}\right)+h_{t}\left(z_{k}\right)\right)\left(\partial_{z_{j}}+\partial_{z_{k}}\right) G_{t}\left(z_{j}, z_{k}\right)\right) & =\mathrm{O}\left(\frac{t}{N} b^{-4} N^{2 \sigma+\varepsilon}\right)\|f\|_{\infty, 3, b}^{2} \\
& =\mathrm{O}\left(t b^{-2} N^{2 \sigma+\varepsilon}\right)\|f\|_{\infty, 3, b}^{2}
\end{aligned}
$$

Collecting the error terms and using 8.32 and $b \geq \theta$, we get the error terms

$$
N^{-1 / 2+3 \sigma+\varepsilon} b^{-1}\|f\|_{3, b}+t N^{\varepsilon} b^{-2} N^{2 \sigma}\|f\|_{3, b}^{2}+N^{-1 / 2+\varepsilon} b\|f\|_{4, b} .
$$

This concludes the proof.

Lemma 8.8. Recall assumption (8.18) and that $h_{t}$ is defined in 8.17). For any $0 \leq|u| \leq \mathrm{O}(t)$ we have the estimate

$$
\mathbb{E}_{V_{t}}^{G_{t}}\left(A_{V_{t}}^{h_{t},-}-A_{V+u f}^{h,-}\right)=\mathrm{O}\left(N^{-1 / 2+3 \sigma+\varepsilon} b^{-1}\right)\|f\|_{\infty, 3, b}+\mathrm{O}\left(t N^{2 \sigma+\varepsilon} b^{-2}\right)\|f\|_{\infty, 3, b}^{2} .
$$

An analogous estimate holds with $\mathbb{E}_{V_{t}}^{G_{t}}$ replaced by $\mathbb{E}_{V}^{\mathcal{C}}$.

Proof. To simplify notation, we set $u=0$ in the following proof as the general case is proved in the same way. By definition,

$$
A_{V_{t}}^{h_{t},-}-A_{V}^{h,-}=\frac{N}{2} \iint_{z \neq w}\left[\Psi_{h_{t}}^{-}(z, w) \tilde{\mu}_{V_{t}}(\mathrm{~d} z) \tilde{\mu}_{V_{t}}(\mathrm{~d} w)-\Psi_{h}^{-}(z, w) \tilde{\mu}_{V}(\mathrm{~d} z) \tilde{\mu}_{V}(\mathrm{~d} w)\right] .
$$

Decompose the integrand into

$$
\left[\Psi_{h_{t}}^{-}-\Psi_{h}^{-}\right](z, w) \tilde{\mu}_{V_{t}}(\mathrm{~d} z) \tilde{\mu}_{V_{t}}(\mathrm{~d} w)+\Psi_{h}^{-}(z, w)\left[\tilde{\mu}_{V_{t}}(\mathrm{~d} z) \tilde{\mu}_{V_{t}}(\mathrm{~d} w)-\tilde{\mu}_{V}(\mathrm{~d} z) \tilde{\mu}_{V}(\mathrm{~d} w)\right] .
$$

To estimate the first term, using that

$$
\partial_{s} \partial h_{s}(z)=\mathrm{O}\left(\|\nabla f\|_{\infty}\left\|\nabla^{3} f\right\|_{\infty}+\left\|\nabla^{2} f\right\|_{\infty}^{2}\right)=\mathrm{O}\left(b^{-4}\right)\|f\|_{\infty, 3, b}^{2},
$$

with high probability with respect to the measure $P_{V_{t}}^{G_{t}}$ we have

$$
\begin{aligned}
& N \iint_{z \neq w}\left[\Psi_{h_{t}}^{-}-\Psi_{h}^{-}\right](z, w) \tilde{\mu}_{V_{t}}(\mathrm{~d} z) \tilde{\mu}_{V_{t}}(\mathrm{~d} w) \\
& \leq N \int_{0}^{t} \mathrm{~d} s \iint_{z \neq w}\left|\partial_{s} \partial h_{s}(z)\right| \mathbb{1}(|z-w| \leq \theta) \tilde{\mu}_{V_{t}}(\mathrm{~d} z) \tilde{\mu}_{V_{t}}(\mathrm{~d} w) \\
& \leqslant \mathrm{O}\left(t N^{2 \sigma+\varepsilon} b^{-2}\right)\|f\|_{\infty, 3, b}^{2}
\end{aligned}
$$


where we used the local density estimate Proposition 8.5, and the factor $\theta^{2} b^{2}$ comes from the integration restriction that $z$ is in the support of $f$ and $|w-z| \lesssim \theta$.

Similarly we can estimate the second term in (8.34). We start with the bound that, with high probability,

$$
\begin{aligned}
& N \iint \Psi_{h_{t}}^{-}(z, w)\left[\tilde{\mu}_{V_{t}}(\mathrm{~d} z)-\tilde{\mu}_{V+t f}(\mathrm{~d} z)\right] \tilde{\mu}_{V_{t}}(\mathrm{~d} w) \\
& =\mathrm{O}(N) \iint \Psi_{h_{t}}^{-}(z, w) t \Delta F(z) m(\mathrm{~d} z) \tilde{\mu}_{V_{t}}(\mathrm{~d} w)=\mathrm{O}\left(N^{1+\varepsilon} \theta^{2} b^{2}\right)\left\|\nabla h_{t}\right\|_{\infty}\|t \Delta F\|_{\infty} \\
& =\mathrm{O}\left(N^{-1 / 2+3 \sigma+\varepsilon}\right)\|f\|_{\infty, 2, b} t b^{-3}\|f\|_{\infty, 4, b}=\mathrm{O}\left(N^{-1 / 2+3 \sigma+\varepsilon}\right) b^{-1}\|f\|_{\infty, 2, b}
\end{aligned}
$$

where we have used Lemma 8.6 to bound $\left\|\nabla h_{t}\right\|_{\infty}\|t \Delta F\|_{\infty}$ and assumption (8.18) in the last step. Similar argument also leads to

$$
\begin{array}{r}
N \iint \Psi_{h_{t}}^{-}(z, w)\left[\tilde{\mu}_{V}(\mathrm{~d} z)-\tilde{\mu}_{V+t f}(\mathrm{~d} z)\right] \tilde{\mu}_{V_{t}}(\mathrm{~d} w)=\mathrm{O}(N t) \iint \Psi_{h_{t}}^{-}(z, w) \Delta f(z) m(\mathrm{~d} z) \tilde{\mu}_{V_{t}}(\mathrm{~d} w) \\
=\mathrm{O}\left(N^{1+\varepsilon} t \theta^{2} b^{2}\right)\left\|\nabla h_{t}\right\|_{\infty}\|\Delta f\|_{\infty}=\mathrm{O}\left(N^{2 \sigma+\varepsilon} t b^{-2}\right)\|f\|_{\infty, 2, b}^{2} .
\end{array}
$$

Collecting all these bounds and using $\|f\|_{\infty, 2, b}^{2} \leq\|f\|_{\infty, 3, b}^{2}$, we have proved Lemma 8.8. Notice that we have used assumption (8.18) in the proof so that the right side of 8.32 does not involve $\|f\|_{4, b}$. This completes the proof.

8.4. Proof of Proposition 8.1. The proof of Proposition 8.1 follows the strategy in [6] by first estimating the sum of the long and short range angle terms with the local law Proposition 8.5 .

Lemma 8.9. For any $\varepsilon>0$, uniformly in $0 \leqslant t \ll 1$ with $t\|\Delta f\|_{\infty} \ll 1$, we have

$$
\mathbb{E}_{V_{t}}^{G_{t}}\left(A_{V_{t}}^{g,+}+A_{V_{t}}^{g,-}\right)=\mathrm{O}\left(N^{\varepsilon}\right) b\|g\|_{\infty, 2, b}
$$

Proof. The proof is exactly the same as that of [6. Lemma 7.5], using the local density estimate Proposition 8.5. Here $A^{-}$corresponds to $t \leqslant N^{-1 / 2+\delta}$ in that proof and $A^{+}$to $t \geqslant N^{-1 / 2+\delta}$.

Inserting these bounds into (8.23), we obtain the following rigidity estimate. This estimate is essentially the same as the rigidity estimate for the Coulomb gas, i.e., [6, Theorem 1.2]. The only difference is that the estimate is with respect to the Coulomb gas with an angle term, i.e., the measure $\mathbb{P}_{V_{t}}^{G_{t}}$.

Proposition 8.10. Assume the same conditions as in Proposition 8.5. For any $\varepsilon>0, s \in(0,1 / 2)$, for any $f$ supported in a ball of radius $b=N^{-s}$ contained in $S_{V}$ with distance of order 1 to $\partial S_{V}$,

$$
X_{f} \prec\|f\|_{\infty, 4, b}
$$

with respect to the measure $\mathbb{P}_{V_{t}}^{G_{t}}$.

Proof. The proof is exactly the same as the proof of [6, Theorem 1.2].

Finally, using this rigidity estimate instead of the local law of Proposition 8.5, we obtain the following improved bound on $A^{+}$, which consists of correlations at range longer than $N^{-1 / 2+\sigma}$. The proof of Lemma 8.11 uses a loop equation and will be given in Section A.4, where a systematical treatment of loop equation will be presented. We remark that a similar estimate for Coulomb gas was already proved in [6]. 
Lemma 8.11 (Refined estimate on the long range angle term). For any $\varepsilon>0$, uniformly in $0 \leqslant t \ll 1$ with $t\|\Delta f\|_{\infty} \ll 1$ and for any function $g$, we have

$$
\mathbb{E}_{V_{t}}^{G_{t}}\left(A_{V_{t}}^{g,+}\right)=\mathrm{O}\left(N^{-\sigma+\varepsilon}\right) b\|g\|_{\infty, 2, b} .
$$

In particular, when $g=h_{t}$, the last term is bounded by $\mathrm{O}\left(N^{-\sigma+\varepsilon}\right)\|f\|_{3, b}$. For a Coulomb gas satisfying (2.32) a similar estimate holds, i.e.,

$$
\mathbb{E}_{V+t f}^{\mathcal{C}}\left(A_{V+t f}^{g,+}\right)=\mathrm{O}\left(N^{-\sigma+\varepsilon}\right) b\|g\|_{\infty, 2, b} .
$$

Proof of Proposition 8.1. Proposition 8.1 follows immediately from 8.22 and Lemma 8.11.

8.5. Concentration of the angle term (macroscopic case): proof of Proposition 8.2, In this subsection, we assume $b$ is of order 1 . The main input of the proof of Proposition 8.2 is the following estimate of large deviations type, which is a direct consequence of Theorem 1.1 .

Corollary 8.12. Assume that $V$ satisfies the conditions of Remark 4.2. Let $0<t \ll 1$ and $\kappa<1 / 24$. Then for any $f \in \mathscr{C}^{5}$ whose support is contained in $S_{V}$ and has distance of order 1 to the boundary of $S_{V}$, assuming that $t\|\Delta f\|_{\infty} \ll 1$, we have

$\frac{1}{t \beta N} \log \mathbb{E}_{V} \mathrm{e}^{-\beta t N X_{V}^{f}}=\frac{t N}{8 \pi} \int|\nabla f|^{2} \mathrm{~d} m+\left(\frac{1}{2}-\frac{1}{\beta}\right) Y_{V}^{f}+\mathrm{O}\left(N^{-\kappa} / t\right)\left(1+\|\Delta f\|_{\infty, 3}\right)^{2}+\mathrm{O}(t)\|\Delta f\|_{\infty}^{2}$.

Proof. By Theorem 1.1, we have

$$
\begin{aligned}
\frac{1}{t \beta N} \log \mathbb{E}_{V} \mathrm{e}^{-\beta t N X_{V}^{f}} & =N \int f \mathrm{~d} \mu_{V}-\frac{N}{t}\left(I_{V+t f}-I_{V}\right) \\
& +\left(\frac{1}{2}-\frac{1}{\beta}\right) \frac{1}{t}\left(\int \rho_{V+t f} \log \rho_{V+t f}-\int \rho_{V} \log \rho_{V}\right)+\mathrm{O}\left(t^{-1} N^{-\kappa}\right),
\end{aligned}
$$

with an $f$-dependent error term. More precisely, by Remark 4.2 , with $V$ fixed, the $f$-dependence of the error term can be taken to be $\mathrm{O}\left(t^{-1} N^{-\kappa}\right)\left(1+\|\Delta f\|_{\infty, 3}\right)^{2}$.

Using that $\rho_{V}=\frac{1}{4 \pi} \Delta V \mathbb{1}_{S_{V}}$ and $\rho_{V+t f}=\frac{1}{4 \pi}(\Delta V+\Delta f) \mathbb{1}_{S_{V}}$ for $f$ with compact support contained in $S_{V}$ such that $t \Delta f<\Delta V$ in its support, an explicit calculation (see, e.g., 6 , Proposition 3.1]) shows that

$$
I_{V+t f}-I_{V}=t \int f \mathrm{~d} \mu_{V}-\frac{t^{2}}{8 \pi} \int|\nabla f|^{2} \mathrm{~d} m
$$

and that

$$
\begin{aligned}
\frac{1}{t}\left(\int \rho_{V+t f} \log \rho_{V+t f}-\int \rho_{V} \log \rho_{V}\right) & =\frac{1}{4 \pi} \int \Delta f \log \rho_{V}+\frac{1}{t} \int \rho_{V+t f} \log \left(\frac{\rho_{V+t f}}{\rho_{V}}\right) \\
& =\frac{1}{4 \pi} \int \Delta f \log \rho_{V}+\mathrm{O}\left(t \int(\Delta f)^{2}\right),
\end{aligned}
$$

where for the last equality we expanded $\log (1+t \Delta f / \Delta V)$ to first order and used $\int \Delta f=0$. Equations 8.44 and 8.45 in 8.43 conclude the proof.

Proof of Proposition 8.2. Let $\kappa$ be as in Corollary 8.12 and write $W=V-t f$. By an elementary identity as in (8.4), we have

$$
\frac{1}{t \beta N} \log \mathbb{E}_{V} \mathrm{e}^{t \beta N \hat{A}_{V+u f}^{f}}=\frac{1}{t \beta N}\left(\log \mathbb{E}_{W} \mathrm{e}^{-\beta N t\left(X_{V}^{f}-\hat{A}_{V+u f}^{f}\right)}-\log \mathbb{E}_{W} \mathrm{e}^{-\beta N t X_{V}^{f}}\right) .
$$


We can replace $X_{V}^{f}$ by $X_{W}^{f}$ in the two exponents in the above equation since $X_{V}^{f}-X_{W}^{f}$ is a constant which cancels in the above expression. Also, $\hat{A}_{V+u f}^{f}=\hat{A}_{W+(t+u) f}^{f}$. By Proposition 8.1.

$$
\frac{1}{t \beta N} \log \mathbb{E}_{W} \mathrm{e}^{-\beta N t\left(X_{W}^{f}-\hat{A}_{W+(t+u) f}^{f}\right)}=-\frac{1}{2} Y_{W}^{f}+N^{\varepsilon} \mathrm{O}\left(t N^{2 \sigma}+N^{-\sigma}+N^{-1 / 2+3 \sigma}\right)\left(1+\|f\|_{\infty, 4}\right)^{2} .
$$

By Corollary 8.12 with $V$ replaced by $W$, we can estimate the last term $\log \mathbb{E}_{W} \mathrm{e}^{-\beta N t X_{W}^{f}}$. Recall from (8.28) that $Y_{W}^{f}=Y_{V}^{f}+\mathrm{O}\left(t \int|\Delta f|^{2} \mathrm{~d} m\right)$. Putting all these bounds together, we have arrived at

$$
\frac{1}{t \beta N} \log \mathbb{E}_{V} \mathrm{e}^{t \beta N \hat{A}_{V+u f}^{f}}=-\frac{1}{2} Y_{V}^{f}+N^{\varepsilon} \mathrm{O}\left(t N^{2 \sigma}+N^{-\sigma}+t^{-1} N^{-\kappa}+N^{-1 / 2+3 \sigma}\right)\left(1+\|f\|_{\infty, 5}\right)^{2} .
$$

This proves (8.3) in the specific case $t=N^{-4 \sigma}=N^{-2 / 3 \kappa}$. Moreover, the bound also holds as claimed for smaller $t$ by the monotonicity of $t \mapsto t^{-1} \log \mathbb{E}\left(\mathrm{e}^{t X}\right)$ applied with the choice $X=\beta N\left(\hat{A}_{V+u f}^{f}+\frac{1}{2} Y_{V}^{f}\right)$.

8.6. CLT for mesoscopic test functions. To extend the proof of the central limit theorem to test functions on mesoscopic scales, it suffices to prove the following estimate for the local angle term. Recall that $\hat{A}_{V}^{f}$ was defined in (8.1) and satisfies 8.13).

Proposition 8.13. Suppose that $V$ satisfies the conditions 1.4 and 1.10 . Let $s \in\left(0, \frac{1}{2}\right)$ and assume that $f$ is supported in a ball of radius $b=N^{-s}$ contained in $S_{V}$ with distance of order 1 to the boundary $\partial S_{V}$. Then there exists $\tau=\tau(s)>0$ such that with high probability under the measure $P_{V}^{\mathcal{C}}$,

$$
\left|\hat{A}_{V}^{f}+\frac{1}{2} Y_{V}^{f}\right| \prec\left(N b^{2}\right)^{-\tau / 3}\|f\|_{\infty, 5, b} .
$$

This proposition can be proved by following the strategy used in the proof of Proposition 8.2 , after conditioning on the particles outside a mesoscopic ball with radius of order $b$ containing the support of $f$. Before implementing this, we complete the proof of Theorem 1.2 using (8.46).

Proof of Theorem 1.2 for mesoscopic test functions. We apply 8.23 and we need to estimate the term $\frac{1}{t} \operatorname{Re} \int_{0}^{t} \mathbb{E}_{V+s f}^{\mathcal{C}}\left(A_{V+s f}^{h_{s},-}+A_{V+s f}^{h_{s},+}\right) \mathrm{d} s$ on right hand side of $(8.23)$. The term $A^{+}$is again bounded by Lemma 8.11. To estimate the expectation of $A^{-}$, we now use 8.46) which implies that with high probability

$$
A_{V+s f}^{h_{s},-}=-\frac{1}{2} Y_{V+s f}^{f}+\mathrm{O}\left(M^{-\tau / 3+\varepsilon}\right)\|f\|_{\infty, 5, b}=-\frac{1}{2} Y_{V}^{f}+\mathrm{O}\left(M^{-\tau / 3+\varepsilon}+s b^{-2}\right)\|f\|_{\infty, 5, b}
$$

where we have used $Y_{V+s f}^{f}=Y_{V}^{f}+\mathrm{O}\left(s \int|\Delta f|^{2} \mathrm{~d} m\right)$ as in 8.45$)$. Clearly, the high probability estimate immediately implies the same estimate under expectation. Integrating $s$ from 0 to $t$, this implies an estimate on the term $\frac{1}{t} \operatorname{Re} \int_{0}^{t} \mathbb{E}_{V+s f}^{\mathcal{C}}\left(A_{V+s f}^{h_{s},-}+A_{V+s f}^{h_{s},+}\right) \mathrm{d} s$. Inserting this estimate into 8.23 , we have completed the proof of Theorem 1.2 .

In the remainder of this section, we prove Proposition 8.13 . For this, we use the approach of local conditioning of [6] and then proceed as in the proof of Proposition 8.2. The local conditioning and its properties are given in Section 2.6. Relative to the conditioned measure, for $f$ compactly supported in $S_{W} \subset S_{V}$, the definitions 1.11), 1.12) translate to

$$
X_{N, V}^{f}=X_{M, W}^{f}=\sum_{j} f\left(\tilde{z}_{j}\right)-M \int f \mathrm{~d} \mu_{W}, \quad Y_{V}^{f}=Y_{W}^{f}=\frac{1}{4 \pi} \int \Delta f \log \rho_{W} \mathrm{~d} m,
$$


where $\rho_{W}$ is the density of the absolutely continuous part of $\mu_{W}$; inside the support of $f$, this density equals that of $\mu_{V}$ up to rescaling. The angle term relative to the conditioned measure is

$$
\hat{A}_{V}^{f}=\hat{A}_{W}^{f}=\frac{M}{2} \operatorname{Re} \iint_{z \neq w} \Psi_{h_{W}}^{-}(z, w) \tilde{\mu}_{W}(\mathrm{~d} z) \tilde{\mu}_{W}(\mathrm{~d} w), \quad h_{W}=\frac{\bar{\partial} f(z)}{\partial \bar{\partial} W(z)} .
$$

The following proposition is a conditioned version of Proposition 8.2 . Note that Lemma 2.6 implies that the assumptions of this proposition holds with high probability. Thus by the Markov inequality, the following Proposition 8.14 immediately implies Proposition 8.13 .

Proposition 8.14. Let $W$ be the conditional potential defined above and assume that it satisfies the conclusions of Lemma 2.6. Choosing the local angles cutoff $\theta=M^{-1 / 2+\sigma}$ with $\sigma=\tau / 6$, for any $0 \leq t \leq M^{-2 \tau / 3}$ we have

$$
\frac{1}{t \beta M} \log \mathbb{E}_{W} \mathrm{e}^{t \beta M\left(\hat{A}_{W}^{f}+\frac{1}{2} Y_{W}^{f}\right)}=\mathrm{O}\left(M^{-\tau / 3}\right)\left(1+\|f\|_{\infty, 5, b}\right)^{2}
$$

To prove Proposition 8.14, we need a version of Theorem 1.1 for the conditioned measure. Recall that $\mu_{W}$ denotes the unique minimizer of the energy functional

$$
\mathcal{I}_{W}(\mu)=\iint \log \frac{1}{|z-w|} \mu(\mathrm{d} z) \mu(\mathrm{d} w)+\int W(z) \mu(\mathrm{d} z)
$$

defined for probability measures supported in $B$, and that its minimum value is $I_{W}=\mathcal{I}_{W}\left(\mu_{W}\right)$.

Theorem 8.15. Let $W$ be the conditional potential defined above and assume that it satisfies the conclusions of Lemma 2.6. Then there exists $\tau>0$ (depending on the constant $\tau$ in Lemma 2.6 but possibly smaller; here we have abused the notation and use the same symbol $\tau$ ) such that with $\zeta^{\mathcal{C}, \beta} \in \mathbb{R}$ defined in Theorem 1.1 .

$$
\begin{aligned}
\frac{1}{\beta M} \log \int_{B^{M}} \mathrm{e}^{-\beta H_{W}^{\mathcal{C}}(\mathbf{z})} m^{\otimes M}(\mathrm{~d} \mathbf{z}) & \\
& =-M I_{W}+\zeta^{\mathcal{C}, \beta}+\frac{1}{2} \log M+\left(\frac{1}{2}-\frac{1}{\beta}\right) \int_{B} \rho_{W}(z) \log \rho_{W}(z) m(\mathrm{~d} z)+\mathrm{O}\left(M^{-\tau}\right),
\end{aligned}
$$

where $\rho_{W}$ is the density of the absolutely continuous part of $\mu_{W}$.

Proof. We apply the local version of Theorem 1.1, i.e., Theorem 4.2, to the conditional Coulomb gas satisfying the properties stated in Lemma 2.6. To apply Theorem 4.2, we first rescale and center the domain $B$, which is a disk of radius $b$, to the unit disk $\mathbb{D}$ with center at 0 . Since the translation is trivial, we will assume that the center of $B$ is already at the origin. Denote the rescaling by $\mathbf{z}=b \mathbf{u}$ and define the new Hamiltonian $\hat{H}_{W}^{\mathcal{C}}(\mathbf{u})$ through the identity

$$
\int_{B^{M}} \mathrm{e}^{-\beta H_{W}^{\mathcal{C}}(\mathbf{z})} m^{\otimes M}(\mathrm{~d} \mathbf{z})=\int_{\mathbb{D}^{M}} \mathrm{e}^{-\beta \hat{H}_{W}^{\mathcal{C}}(\mathbf{u})} m^{\otimes M}(\mathrm{~d} \mathbf{u}) .
$$

Hence $\hat{H}_{W}^{\mathcal{C}}(\mathbf{u})$ is a Coulomb gas with external potential $\tilde{W}(u)=W(b u)$ up to a constant. More precisely,

$$
\hat{H}_{W}^{\mathcal{C}}(\mathbf{u})=H_{W}^{\mathcal{C}}(\mathbf{u} / b)-2 M \beta^{-1} \log b=H_{\tilde{W}}^{\mathcal{C}}(\mathbf{u})-M(M-1) \log b-\frac{1}{\beta} M \log b^{2} .
$$


By Theorem 4.2, there exists $\tau>0$ such that

$$
\begin{aligned}
& \frac{1}{\beta M} \log \int_{B^{M}} \mathrm{e}^{-\beta H_{W}^{\mathcal{C}}(\mathbf{z})} m^{\otimes M}(\mathrm{~d} \mathbf{z})=\frac{1}{\beta M} \log \int_{\mathbb{D}^{M}} \mathrm{e}^{-\beta H_{M, \tilde{W}}(\mathbf{u})} m^{\otimes M}(\mathrm{~d} \mathbf{u})+M \log b-\left(\frac{1}{2}-\frac{1}{\beta}\right) \log b^{2} \\
& =-M\left(I_{\tilde{W}}-\log b\right)+\frac{1}{2} \log M+\left(\frac{1}{2}-\frac{1}{\beta}\right)\left[\int_{\mathbb{D}} \rho_{\tilde{W}}(u) \log \rho_{\tilde{W}}(u) m(\mathrm{~d} u)-\log b^{2}\right]+\mathrm{O}\left(M^{-\tau}\right) .
\end{aligned}
$$

Recall the normalization conditions for the densities $\int \rho_{\tilde{W}}(u) m(\mathrm{~d} u)=1=\int \rho_{W}(z) m(\mathrm{~d} z)$. Hence $\rho_{\tilde{W}}(u)=\rho_{W}(b u) b^{2}$ and we have

$$
\int_{\mathbb{D}} \rho_{\tilde{W}}(u) \log \rho_{\tilde{W}}(u) m(\mathrm{~d} u)-\log b^{2}=\int_{B} \rho_{W}(z) \log \rho_{\tilde{W}}(z) m(\mathrm{~d} z) .
$$

A similar argument shows that $\left(I_{\tilde{W}}-\log b\right)=I_{W}$. We have thus proved Theorem 8.15 .

Proof of Proposition 8.14. By assumption, the potential $W$ satisfies the conditions of Theorem 4.2, and therefore the assumptions of Proposition 8.1. Together with using Proposition 8.15 to replace Theorem 1.1, the proof of Proposition 8.14 follows in exactly the same way as that of Proposition 8.2 .

As in the proof of Theorem 8.15, one can also derive a conditioned version of the CLT, stated below; we omit the details of the proof.

Theorem 8.16. Suppose $W$ is the conditional potential defined above and assume that it satisfies the conclusions of Lemma 2.6. Then for any $\beta>0, c \in(0,1)$ and large $C>0$, there a positive constant $\tau>0$ such that the following holds. For any $f: \mathbb{C} \rightarrow \mathbb{R}$ supported in the ball with same center as $B$ and radius $b(1-c)$ and satisfying $\|f\|_{4, b}<C$, and for any $0 \leqslant \lambda \leq M^{1-2 \tau}$, we have

$$
\frac{1}{\beta \lambda} \log \left(\mathbb{E}_{M, W, \beta}^{\mathcal{C}} \mathrm{e}^{-\lambda \beta\left(X_{W}^{f}-\left(\frac{1}{\beta}-\frac{1}{2}\right) Y_{W}^{f}\right)}\right)=\frac{\lambda}{8 \pi} \int|\nabla f(z)|^{2} m(\mathrm{~d} z)+\mathrm{O}\left(M^{-\tau}\right) .
$$

Note that the measure associated to the external potential $W+\frac{\lambda}{M} f$ is a perturbation of the original measure provided that

$$
|\lambda \Delta f| \ll|M \Delta W|=|N \Delta V| .
$$

Our assumptions $\|f\|_{4, b}<C$ and $\lambda \leq M^{1-2 \tau}$ guarantee this condition. Also note that, in the context of the above Theorem 8.16, our test function has shrinking support so that

$Y_{W}^{f}=\frac{1}{4 \pi} \int \Delta f(z) \log \rho_{W}(z) \mathrm{d} m(z)=\frac{1}{4 \pi} \int \Delta f(z) \log \frac{\Delta V(z)}{\Delta V\left(z_{0}\right)} \mathrm{d} m(z)=\mathrm{O}(b)\|f\|_{\infty, b, 2}\|V\|_{S_{V}, \infty, 3}=\mathrm{o}(1)$,

where we used (2.30) and denoted the center of $\mathcal{J}$ by $z_{0}$. Thus Theorem 8.16, with $\lambda$ of order 1 , implies there is no shift of the mean in the convergence to the Gaussian free field for mesoscopic observables:

$$
X_{W}^{f} \underset{N \rightarrow \infty}{\stackrel{(\mathrm{d})}{\longrightarrow}} \mathscr{N}\left(0, \frac{1}{4 \pi \beta} \int|\nabla f|^{2}\right)
$$

\section{Appendix A Rigidity estimates for Yukawa gas on torus}

In this appendix, we prove Theorem 2.4 and Proposition 2.5. The proofs use the same ideas as that of [6, Theorem 1.2]. We will also prove Lemma 8.11 by the same argument. 
A.1. Loop equation. As the first step, we state the loop equation for the Yukawa gas on the torus. Given a function $v: \mathbb{T} \rightarrow \mathbb{R}$, the function $W_{V}^{U^{\ell}, v}: \mathbb{T}^{N} \rightarrow \mathbb{C}$ was defined by (8.6). For simplicity of notation, we denote it by $W_{V}^{v}(\mathbf{z})$ in this section, i.e.,

$$
W_{V}^{v}(\mathbf{z})=-\sum_{j \neq k}\left(v\left(z_{j}\right)-v\left(z_{k}\right)\right) \partial U^{\ell}\left(z_{j}-z_{k}\right)+\frac{1}{\beta} \sum_{j} \partial_{j} v\left(z_{j}\right)-N \sum_{j} v\left(z_{j}\right) \partial V\left(z_{j}\right) .
$$

By Lemma 8.3. $\mathbb{E}_{V}^{U^{\ell}}\left(W_{V}^{v}\right)=0$ since the Yukawa interaction $Y^{\ell}$ (and therefore $U^{\ell}$ ) are functions of $z_{j}-z_{k}$. Given $q: \mathbb{C} \rightarrow \mathbb{R}$ supported in $S_{V}$, further abbreviate

$$
h(z)=\frac{1}{\pi} \frac{\bar{\partial} q(z)}{\rho_{V}(z)}, \quad g(z)=\frac{1}{\pi} \frac{q(z)}{\rho_{V}(z)},
$$

where $\rho_{V}$ denotes the density of $\mu_{V}(2.14)$ with respect to the Lebesgue measure. The following lemma extends Lemma 8.4 from the Coulomb gas to the Yukawa gas.

Lemma A.1. For any $q: \mathbb{T} \rightarrow \mathbb{R}$ of class $\mathscr{C}^{2}$ supported on $S_{V}$ and $\mathbf{z} \in \mathbb{T}^{N}$, recall $X_{V}^{q}$ defined in (1.11). Then we have

$$
\begin{aligned}
X_{V}^{q}=- & \frac{1}{N} W_{V}^{h}(\mathbf{z})+\frac{1}{N \beta} \sum_{k} \partial h\left(z_{k}\right)+N \iint_{z \neq w}(h(z)-h(w)) \partial U^{\ell}(z-w) \tilde{\mu}_{V}(\mathrm{~d} z) \tilde{\mu}_{V}(\mathrm{~d} w) \\
& +\frac{N m^{2}}{2} \iint_{z \neq w} g(w) U^{\ell}(z-w) \tilde{\mu}_{V}(\mathrm{~d} z) \mu_{V}(\mathrm{~d} w),
\end{aligned}
$$

where $m=\ell^{-1}$ an $h$ is defined in A.2. Thus for any smooth enough $f: \mathbb{T} \rightarrow \mathbb{R}$ with

$$
q=f-\int_{\mathbb{T}} f \mathrm{~d} m-m^{2} \Delta^{-1}\left(f-\int_{\mathbb{T}} f \mathrm{~d} m\right)
$$

supported in $S_{V}$, where $\Delta$ is the Laplacian on the torus, we have

$$
X_{V}^{f}=-\frac{1}{N} W_{V}^{h}(\mathbf{z})+\frac{1}{N \beta} \sum_{k} \partial h\left(z_{k}\right)+N \iint_{z \neq w}(h(z)-h(w)) \partial U^{\ell}(z-w) \tilde{\mu}_{V}(\mathrm{~d} z) \tilde{\mu}_{V}(\mathrm{~d} w) .
$$

Proof. As in the proof of Lemma 8.4, we have

$$
\begin{aligned}
& 2 \int \partial U^{\ell}(z-w) \mu_{V}(\mathrm{~d} w)=\partial V(z) \\
& q(z)=\frac{1}{2 \pi} \int\left(-4 \partial \bar{\partial} q(w)+m^{2} q(w)\right) U^{\ell}(z-w) m(\mathrm{~d} w) \\
& \quad=\frac{1}{2 \pi} \int\left(4 \bar{\partial} q(w) \partial U^{\ell}(z-w)+m^{2} q(w) U^{\ell}(z-w)\right) m(\mathrm{~d} w),
\end{aligned}
$$

where again the first equation holds for $z \in S_{V}$ by the Euler-Lagrange equation, the second equation holds by the definition of the Yukawa potential as the Green's function of $-\Delta+m^{2}$ and integration by parts - the boundary term vanishes by periodicity. We therefore have

$$
\begin{aligned}
X_{V}^{q} & =2 \sum_{j} \int h(w) \partial U^{\ell}\left(z_{j}-w\right) \mu_{V}(\mathrm{~d} w)+\frac{m^{2}}{2} \sum_{j} \int g(w) U^{\ell}\left(z_{j}-w\right) \mu_{V}(\mathrm{~d} w) \\
& -2 N \iint h(w) \partial U^{\ell}(z-w) \mu_{V}(\mathrm{~d} w) \mu_{V}(\mathrm{~d} z)-\frac{N m^{2}}{2} \iint g(w) U^{\ell}(z-w) \mu_{V}(\mathrm{~d} w) \mu_{V}(\mathrm{~d} z) \\
& =2 N \iint(h(w)-h(z)) \partial U^{\ell}(z-w) \hat{\mu}(\mathrm{d} z) \mu_{V}(\mathrm{~d} w)+\sum_{j} h\left(z_{j}\right) \partial V\left(z_{j}\right) \\
& -N \iint(h(w)-h(z)) \partial U^{\ell}(z-w) \mu_{V}(\mathrm{~d} w) \mu_{V}(\mathrm{~d} z)+\frac{N m^{2}}{2} \iint g(w) U^{\ell}(z-w) \tilde{\mu}_{V}(\mathrm{~d} z) \mu_{V}(\mathrm{~d} w) .
\end{aligned}
$$


In the first equation we used (A.2) and A.7), and in the second equation we used (A.6). Since the integrands in the double integrals are symmetric, we arrive at

$$
\begin{array}{r}
X_{V}^{q}=-\frac{1}{N} \sum_{j \neq k}\left(h\left(z_{j}\right)-h\left(z_{k}\right)\right) \partial U^{\ell}\left(z_{j}-z_{k}\right)+N \iint_{z \neq w}(h(z)-h(w)) \partial U^{\ell}(z-w) \tilde{\mu}_{V}(\mathrm{~d} z) \tilde{\mu}_{V}(\mathrm{~d} w) \\
+\sum_{j} h\left(z_{j}\right) \partial V\left(z_{j}\right)+\frac{N m^{2}}{2} \iint_{z \neq w} g(w) U^{\ell}(z-w) \tilde{\mu}_{V}(\mathrm{~d} z) \mu_{V}(\mathrm{~d} w),
\end{array}
$$

which is equivalent to A.3.

For the consequence, note that moving the last term on the right-hand side to the left-hand side, the left-hand side becomes $X^{f}$ with

$$
f(z)=q(z)-\frac{m^{2}}{2 \pi} \int q(w) U^{\ell}(z-w) m(\mathrm{~d} w)=((1-K) q)(z),
$$

where

$$
1-K=1-\left(1-\ell^{2} \Delta\right)^{-1}=\frac{\ell^{2} \Delta}{\ell^{2} \Delta-1}, \quad(1-K)^{-1}=1-m^{2} \Delta^{-1} .
$$

Therefore, given $f$ as in the assumption, we can choose $q=f-m^{2} \Delta^{-1} f$.

Finally, since $\int \mathrm{d} \mu_{V}=1$, we have $X_{V}^{f}=X_{V}^{f-c}$ for any constant $c$. Hence the assumption $\int_{\mathbb{T}} f \mathrm{~d} m=0$ is trivial to remove.

A.2. Estimate on two-point correlations. For the analysis of the loop equation, we need weak decorrelation estimates for two-point observables. The following simple estimate based on Taylor expansion and the boundedness of the local density. Let $\omega_{t}$ be a nonnegative mollifier such that $\int \omega_{t}(z) \mathrm{d} z=1, \omega_{t}$ has support in a square of side length $t$, and $\left\|\omega_{t}^{(n)}\right\|_{\infty} \leqslant C_{n} t^{-2-n}$ for all $n \geqslant 0$. In the lemma below $g$ is an arbitrary function on $\mathbb{T}^{2}$, unrelated to the normalization A.2.

Lemma A.2. Consider the Yukawa gas on the unit torus with range $N^{-1 / 2+\sigma} \leqslant \ell \leqslant 1$. Recall the definition (2.24) and the notations of Proposition 2.5. Fix a scale $t$ with $N^{-1 / 2+\sigma} \leqslant t \leqslant N^{-\sigma}$. Then for any fixed $p \in \mathbb{N}$ and $\varepsilon>0$, there exist functions $\left|F^{(j, \mathbf{k})}(x, y)\right|=\mathrm{O}\left(\left|\nabla^{j} g(x, y)\right|\right)$ such that the following bound holds with high probability:

$$
\begin{aligned}
& \iint g(z, w) \tilde{\mu}(\mathrm{d} z) \tilde{\mu}(\mathrm{d} w)=\sum_{j=1}^{p} \sum_{\sum k_{i}=j} \iint F^{(j, \mathbf{k})}(x, y) m(\mathrm{~d} x) m(\mathrm{~d} y) \\
& \times\left(\iint \varphi_{\mathbf{k}}(x, y, z, w) \omega_{t}(z-x) \omega_{t}(w-y) \tilde{\mu}(\mathrm{d} z) \tilde{\mu}(\mathrm{d} w)\right)+\mathrm{O}\left(t^{p}\left\|g_{\mathrm{B}_{t}}^{(p)}\right\|_{1}\right),
\end{aligned}
$$

where $\|\cdot\|_{1}$ is the $L^{1}$-norm over $\mathbb{T} \times \mathbb{T}$, and

$$
\varphi_{\mathbf{k}}(x, y, z, w)=c_{\mathbf{k}}(x-z)^{k_{1}}(\bar{x}-\bar{z})^{k_{2}}(y-w)^{k_{3}}(\bar{y}-\bar{w})^{k_{4}} .
$$

Remark A.3. This lemma uses only that the density is locally bounded w.r.t. the Yukawa gas. In its application, we choose $t$ such that $t^{p}\left\|g_{t}^{(p)}\right\|_{L^{1}(\mathbb{T} \times \mathbb{T})} \leq N^{-\varepsilon p}$. If $g$ is a function smooth at the scale $W$, say, then $t=W N^{-\varepsilon}$ is such a choice.

Proof. By Taylor expansion, for any $(x, y) \in \mathrm{B}(z, t) \times \mathrm{B}(w, t)$ (defined in 2.24) we can write

$$
g(z, w)=\sum_{j=0}^{p-1} \sum_{\sum k_{i}=j, a_{i} \in\{x, \bar{x}, y, \bar{y}\}}\left(\prod_{i=1}^{4}\left(\partial_{a_{i}}\right)^{k_{i}} g(x, y)\right) \varphi_{\mathbf{k}}(x, y, z, w)+R_{p}(z, w: x, y),
$$


where

$$
R_{p}(z, w: x, y)=C \int_{0}^{1}(1-s)^{p} \nabla^{\mathbf{p}} g(x+s(z-x), y+s(w-y)) \mathrm{d} s \varphi_{\mathbf{p}}(x, y, z, w)
$$

is the remainder term. Here $\nabla^{\mathbf{p}}$ is understood as a multi-indices differentiation operators with total degree $p$ and the right-hand side of $R_{p}$ is understood as a sum over all indices with $|\mathbf{p}|=p$. We now rewrite

$$
\iint g(z, w) \tilde{\mu}(\mathrm{d} z) \tilde{\mu}(\mathrm{d} w)=\iint g(z, w) \tilde{\mu}(\mathrm{d} z) \tilde{\mu}(\mathrm{d} w) \omega_{t}(z-x) \omega_{t}(w-y) m(\mathrm{~d} x) m(\mathrm{~d} y),
$$

and insert the equation A.9 into this identity. This gives the sum in A.8 with

$$
\left|F^{(j, \mathbf{k})}(x, y)\right|=\sum_{a_{i} \in\{x, \bar{x}, y, \bar{y}\}}\left(\prod_{i=1}^{4}\left(\partial_{a_{i}}\right)^{k_{i}} g(x, y)\right)=\mathrm{O}\left(\left|\nabla^{j} g(x, y)\right|\right) .
$$

To complete the proof, it remains to bound the remainder term

$$
\begin{aligned}
& \left|\iint R_{p}(z, w: x, y) \tilde{\mu}(\mathrm{d} z) \tilde{\mu}(\mathrm{d} w)\right|=C \mid \iiint_{0}^{1}(1-s)^{p} \nabla^{\mathbf{p}} g(x+s(z-x), y+s(w-y)) \mathrm{d} s \\
& \times\left[\iint \varphi_{\mathbf{p}}(x, y, z, w) \tilde{\mu}(\mathrm{d} z) \tilde{\mu}(\mathrm{d} w) \omega_{t}(z-x) \omega_{t}(w-y)\right] m(\mathrm{~d} x) m(\mathrm{~d} y) \mid \\
& \leq C \iint \mathrm{O}\left(g_{\mathrm{B}_{t}}^{(p)}(x, y)\right) m(\mathrm{~d} x) m(\mathrm{~d} y) \sup _{x, y}\left|\iint \varphi_{\mathbf{p}}(x, y, z, w) \omega_{t}(z-x) \omega_{t}(w-y) \tilde{\mu}(\mathrm{d} z) \tilde{\mu}(\mathrm{d} w)\right| .
\end{aligned}
$$

By the local law, Theorem 2.2, i.e., that the empirical density is bounded with high probability, for any function $k$ supported in a square of size $w \gg N^{-1 / 2}$, we have

$$
\int|k(z)| \hat{\mu}(\mathrm{d} z) \leq C w^{2}\|k\|_{\infty}
$$

and the same estimate holds with $\hat{\mu}$ replaced by $\tilde{\mu}$ since it is trivially true for $\mu_{V}$. Hence

$$
\sup _{x, y}\left|\iint \varphi_{\mathbf{p}}(x, y, z, w) \omega_{t}(z-x) \omega_{t}(w-y) \tilde{\mu}(\mathrm{d} z) \tilde{\mu}(\mathrm{d} w)\right|=\mathrm{O}\left(t^{p}\right) .
$$

This proves the the bound on the error term and completes the proof of the lemma.

A.3. Analysis of loop equation and proof of Theorem 2.4. We next analyze the terms in the loop equation.

Lemma A.4. For any $A>0$, there is a constant $C$ such that for any smooth $f: \mathbb{T} \rightarrow \mathbb{R}$ supported in a ball of radius $b$ with $b \geq N^{-1 / 2}$, there exists $f_{s}$ support in a ball of radius $b_{s}:=b+C s \log N$ for $0 \leq s \leq \log N$ such that $h(z)=\frac{1}{\pi \rho_{V}(z)} \bar{\partial}\left(1-m^{2} \Delta^{-1}\right) f(z)$ can be written as

$$
\begin{gathered}
h(z)=\frac{1}{\pi \rho_{V}(z)}\left(\bar{\partial} f(z)-m^{2} \int_{0}^{\log N} \frac{\mathrm{d} s}{s} \bar{\partial} f_{s}(z)\right)+\mathrm{O}\left(N^{-A}\|f\|_{\infty, 1, b}\right), \\
\left\|f_{s}\right\|_{\infty, k, b_{s}} \leq C(b \wedge s)^{2} N^{\varepsilon}\|f\|_{\infty, k, b} .
\end{gathered}
$$

It is useful to keep in mind that if $f$ is dimensionless then $f_{s}$ has a linear dimension 2. 
Proof. We write

$$
\bar{\partial} \Delta^{-1} f(z)=\int_{0}^{\infty} \mathrm{d} t \mathrm{e}^{t \Delta} \bar{\partial} f(z)=\int_{0}^{M} \mathrm{~d} t \mathrm{e}^{t \Delta} \bar{\partial} f(z)+\int_{M}^{\infty} \mathrm{d} t \mathrm{e}^{-t \Delta} \bar{\partial} f(z) .
$$

Since $\Delta$ has a spectral gap of order one w.r.t mean zero function and $\bar{\partial} f$ is mean zero for any $f$ with compact support, there is $c>0$ such that

$$
\int_{M}^{\infty} \mathrm{d} t \mathrm{e}^{t \Delta} \bar{\partial} f(z) \leq \int_{M}^{\infty} \mathrm{d} t \mathrm{e}^{-c t}\|\bar{\partial} f(z)\|_{2} \leq\|f\|_{\infty, 1, b} \mathrm{e}^{-c M}
$$

We choose $M=(\log N)^{2}$ so that this term is an error term of the form $N^{-A}\|f\|_{1, b}$ for any $A>0$.

The heat kernel on the unit circle is given by

$$
g_{t}(x)=2 \pi \sum_{k \in \mathbb{Z}} k_{t}(x+2 \pi k)=\sqrt{\frac{\pi}{t}} \mathrm{e}^{-x^{2} / 4 t}\left[1+2 \sum_{k \geq 1} e^{-\pi^{2} k^{2} / t} \cosh (\pi k x / t)\right]
$$

where $k_{t}(x)=(4 \pi t)^{-1 / 2} \mathrm{e}^{-x^{2} / 4 t}$. The heat kernel on the two dimensional unit torus is given by $G_{t}(z):=g_{t}(x) g_{t}(y)$. Now change variables $s^{2}=t$. Clearly, the function $G_{s^{2}}$ decays exponentially at scale $s$. Rewrite $G_{s^{2}}=G_{s}^{1}+G_{s}^{2}$ with $G_{s}^{1}(z)=G_{s^{2}}(z) \eta_{s C \log N}(z)$ where $C$ is a large constant and $\eta_{a}(z)$ is a mollifier in a ball of radius $a$ with $\eta_{a}(z)=1$ if $|z| \leq a / 2$ (note that we have changed the subscript from $s^{2}$ to $s$ in $G^{i}$ so that the subscript indicates the scale of the support for the functions $G^{i}$ ). Define

$$
f_{s}(z)=s^{2} \int G_{s}^{1}(z-w) f(w) m(\mathrm{~d} w) .
$$

Clearly, $f_{s}$ is supported in a ball of radius $b_{s}$ and satisfies the bound (A.14). Certainly, when $b_{s} \geq 1, f_{s}$ is supported on the entire torus. The error term involving $G_{s}^{2}$ can be trivially bounded and the constant $A$ can be arbitrary large by choosing $C$ large depending on $A$.

In next lemma, which is parallel to [6, Lemma 7.5], we estimate the last term in A.5. The proof of this lemma uses only the local law (2.18) (In the later application, we only need $V=f / N$.)

Lemma A.5. For any $f: \mathbb{T} \rightarrow \mathbb{R}$, define $h$ as in Lemma A.4, and $G(z, w)=(h(z)-h(w)) \partial U^{\ell}(z-$ $w)$. Then, for the Yukawa gas on the unit torus, we have

$$
\mathbb{E}_{V}^{U^{\ell}}\left(N \iint_{z \neq w} G(z, w) \tilde{\mu}_{V}(\mathrm{~d} z) \tilde{\mu}_{V}(\mathrm{~d} w)\right)=\mathrm{O}\left(N^{\varepsilon}\left(1+\frac{b^{2}}{\ell^{2}}\right)\right)\|f\|_{\infty, 3, b} .
$$

Proof. We first write

$$
\partial U^{\ell}(z)=\sum_{i \leq m} U_{i}(z)+U^{(m)}(z)
$$

where $U_{i}$ is supported in $\ell_{i} / 2 \leq|z| \leq 2 \ell_{i}$ with $\ell_{i}=2^{-i} \ell$ and $U^{(m)}$ is supported in $|z| \leq 2 \ell_{m}=$ $N^{-1 / 2+\varepsilon}$.

Case 1: $U^{(m)}$. For any function $k$ supported in a ball of radius $b$, using the fact that the empirical density is locally bounded up to a factor $N^{\varepsilon}$, with high probability we have

$$
\left|N \iint(\bar{\partial} k(z)-\bar{\partial} k(w)) U^{(m)}(z-w) \tilde{\mu}(\mathrm{d} z) \tilde{\mu}(\mathrm{d} w)\right| \leq N^{1+\varepsilon} \ell_{m}^{2} b^{2}\left\|\nabla^{2} k\right\|_{\infty} \leq N^{1+\varepsilon} \ell_{m}^{2}\|k\|_{\infty, 2, b},
$$


where $\ell_{m}^{2} b^{2}$ comes from the volume in the integration and we have used $|(z-w)| U^{(m)}(z-w) \mid \leq C$.

Recall that $h$ is defined in Lemma A.4. We can apply the previous inequality to $k=f$. To bound the other contribution due to the integral of $\ell^{-2} \bar{\partial} f_{s}$, we use that $f_{s}$ is supported in a ball of size $b_{s}$ and apply A.18 and A.14 to obtain (ignoring the small error $N^{-A}$ from A.13)

$$
\begin{aligned}
& \mid \frac{N}{\ell^{2}} \int_{0}^{\infty} \frac{\mathrm{d} s}{s} \iint\left(\bar{\partial} f_{s}(z)-\right.\left.\bar{\partial} f_{s}(w)\right) U^{(m)}(z-w) \tilde{\mu}(\mathrm{d} z) \tilde{\mu}(\mathrm{d} w) \mid \leq \frac{N^{1+\varepsilon} \ell_{m}^{2}}{\ell^{2}} \int_{0}^{\log N} \frac{\mathrm{d} s}{s}(b \wedge s)^{2}\|f\|_{\infty, 2, b} \\
& \leq N^{2 \varepsilon}\left[1+\left(\sqrt{N} \ell_{m}\right)^{-1}\right]^{2} b\|h\|_{\infty, 2, b} \leq N^{2 \varepsilon}\left[1+\left(\sqrt{N} \ell_{m}\right)^{-1}\right]^{2}\|f\|_{\infty, 3, b} .
\end{aligned}
$$

Case 2: $U_{i}$ for a scale $\ell_{i}:=q \leq \ell$ (notice that $q$ is also used to denote the function in Lemma A.1 and (A.2)). Suppose that $k$ is a function supported in a ball of radius $r$ (note that $r$ can be either smaller or bigger than $\ell$ ). We will prove

$$
\mathbb{E}_{V}^{U^{\ell}}\left(N \iint_{z \neq w}(k(z)-k(w)) U_{i}(z-w) \tilde{\mu}_{V}(\mathrm{~d} z) \tilde{\mu}_{V}(\mathrm{~d} w)\right)=\mathrm{O}\left(N^{2 \varepsilon}\right) A(q)^{2} r\|k\|_{\infty, 2, r}
$$

where $A(q):=1+q \ell^{-1}+(\sqrt{N} q)^{-1}$. Summation over $i \leqslant m$ will give an appropriate bound. Let $M_{i}(z, w)=(k(z)-k(w)) U_{i}(z-w)$. Our goal is to bound $N \iint_{z \neq w} M_{i}(z, w) \tilde{\mu}_{V}(\mathrm{~d} z) \tilde{\mu}_{V}(\mathrm{~d} w)$. Treating $k(z)-k(w)$ as a multiplicative factor, we can apply Lemma A.2 to the function $U_{i}(z-w)$ with the scale $s$ in the lemma replaced by $q N^{-\varepsilon}$. By applying the decomposition (A.8) for fixed and large enough $p$, for each $1 \leqslant j \leqslant p$ and $\mathbf{k}$ we need to estimate

$$
\begin{aligned}
& \iint F^{(j, \mathbf{k})}(x, y) \Omega_{x, y} m(\mathrm{~d} x) m(\mathrm{~d} y) \\
& \Omega_{x, y}=\iint(k(z)-k(w)) \varphi_{\mathbf{k}}(x, y, z, w) \omega_{q}(z-x) \omega_{q}(w-y) \tilde{\mu}(\mathrm{d} z) \tilde{\mu}(\mathrm{d} w),
\end{aligned}
$$

where $\omega_{q}$ is a smooth mollifier at scale $q$ (more precisely, $q N^{-\varepsilon}$ as mentioned in Remark A.3 The reader can follow through this minor change in the following proof).

We now prove that the contribution from $j=0$ in the decomposition of the left hand side of A.19 is bounded by the right hand side of A.19). Recall that when $j=0, \varphi_{\mathbf{k}}(x, y, z, w)=1$ in (A.20). Rewrite $k(z)-k(w)=(k(z)-k(x))+(k(y)-k(w))+(k(x)-k(y))$ and we consider the term involving $k(z)-k(x)$. The other two terms can be estimated in a similar way. Let $R_{x}(z)=(k(z)-k(x)) \omega_{q}(z-x)$. To prove (A.19), we apply the local law (2.18) to have

$$
\left|\int(k(z)-k(x)) \omega_{q}(z-x) \tilde{\mu}(\mathrm{d} z)\right| \leq q N^{-\frac{1}{2}+\varepsilon}\left[\left\|\nabla_{z} R_{x}(z)\right\|_{L_{2}(z)}+\left\|R_{x}(z)\right\|_{L_{2}(z)} \ell^{-1}+N^{-\frac{1}{2}} q\left\|\Delta_{z} R_{x}(z)\right\|_{\infty}\right],
$$

and

$$
\begin{gathered}
\left|\int \omega_{q}(w-y) \tilde{\mu}(\mathrm{d} w)\right| \leq q N^{-\frac{1}{2}+\varepsilon}\left[\left\|\nabla_{w} \omega_{q}(w-y)\right\|_{L_{2}(w)}+\left\|\omega_{q}(w-y)\right\|_{L_{2}(w)} \ell^{-1}+N^{-\frac{1}{2}} q\left\|\Delta_{w} \omega_{q}(w-y)\right\|_{\infty}\right] \\
\leq q^{-1} N^{-\frac{1}{2}+\varepsilon} A(q) . \quad(\text { A. } 22)
\end{gathered}
$$

We now claim that

$$
\begin{gathered}
\left|\iint U_{i}(x, y) m(\mathrm{~d} x) m(\mathrm{~d} y)\left[\left\|\nabla_{z} R_{x}(z)\right\|_{L_{2}(z)}+\left\|R_{x}(z)\right\|_{L_{2}(z)} \ell^{-1}+N^{-1 / 2} q\left\|\Delta_{z} R_{x}(z)\right\|_{\infty}\right]\right| \\
\leq q^{2} r^{2} q^{-1}\left[q^{-1}+\ell^{-1}+N^{-1 / 2} q^{-2}\right] r^{-1}\|k\|_{\infty, 2, r}=A(q) r\|k\|_{\infty, 2, r}
\end{gathered}
$$


If this holds, then the last three inequalities imply the $j=0$ case of $\mathrm{A.19}$.

To prove A.23), we first consider the case $r \geq q$. Recall $\left|U_{i}(x, y)\right| \leq q^{-1} \mathbb{1}(|x-y| \leq q)$. Furthermore,

$$
\left\|\nabla_{z} R_{x}(z)\right\|_{L_{2}(z)} \leq\left\|(k(z)-k(x)) \nabla \omega_{q}(z-x)\right\|_{L_{2}(z)}+\left\|\omega_{q}(z-x) \nabla k(z)\right\|_{L_{2}(z)} .
$$

Clearly, the contribution of this first term involving $\left\|\nabla_{z} R_{x}(z)\right\|_{L_{2}(z)}$ on the left hand side of A.23 is bounded by

$$
\begin{aligned}
& \left|\iint U_{i}(x, y) m(\mathrm{~d} x) m(\mathrm{~d} y)\left\|(k(z)-k(x)) \nabla \omega_{q}(z-x)\right\|_{L_{2}(z)}\right| \\
& \leq q\|\nabla k\|_{\infty} \int m(\mathrm{~d} x) \mathbb{1}(\operatorname{dist}(x, \operatorname{supp} k) \leq q)\left\|q \nabla \omega_{q}(z-x)\right\|_{L_{2}(z)} \leq r^{2}\|\nabla k\|_{\infty} \leq r\|k\|_{\infty, 2, r} .
\end{aligned}
$$

All other terms are bounded similarly and we obtain $($ A.23 in this case $r \geq q$.

We now assume that $r \leq q$. In this case, we view $k(z) \omega_{q}(z-x) \sim k(z) \mathbb{1}_{\operatorname{dist}(x, \operatorname{supp} k) \leq q} q^{-2}$ and obtain

$$
\begin{gathered}
\left|\int k(z) \omega_{q}(z-x) \tilde{\mu}(\mathrm{d} z)\right| \leq \mathbb{1}_{\operatorname{dist}(x, \operatorname{supp} k) \leq q} \frac{r N^{\varepsilon}}{\sqrt{N} q^{2}}\left[\|\nabla k(z)\|_{L_{2}(z)}+\frac{\|k(z)\|_{L_{2}(z)}}{\ell}+\frac{r}{\sqrt{N}}\left\|\Delta_{z} k(z)\right\|_{\infty}\right] \\
\leq \mathbb{1}_{\operatorname{dist}(x, \operatorname{supp} k) \leq q} q^{-2} r N^{-\frac{1}{2}+\varepsilon}\|k\|_{\infty, 2, r},
\end{gathered}
$$

where we have used $r \leq q \leq \ell$ and $N^{-1 / 2} \leq r$. This implies that

$$
\iint U_{i}(x, y) m(\mathrm{~d} x) m(\mathrm{~d} y) \int k(z) \omega_{q}(z-x) \tilde{\mu}(\mathrm{d} z)=\mathrm{O}\left(q r N^{-\frac{1}{2}+\varepsilon}\|k\|_{\infty, 2, r}\right) .
$$

Similar inequality holds with $k(z)$ replaced by $k(x)$. This concludes the proof of (A.23), and therefore the contribution from $j=0$ in (A.19).

One can check in a similar way that the same bound holds for any $j$ since the factor $q^{-j}$ induced by the derivatives on $U_{i}$ is compensated by the size of the function $\varphi_{\mathbf{k}}$. Notice that for all $j$, we need at most two derivatives on $k$; all other derivatives will apply to explicit functions depending on $U_{i}$. Summing over all $j$ and $i$ and using $q \leq C \ell$, we have thus proved

$$
\left|\sum_{i \leq m} N \iint_{z \neq w} M_{i}(z, w) \tilde{\mu}_{V}(\mathrm{~d} z) \tilde{\mu}_{V}(\mathrm{~d} w)\right| \leq N^{2 \varepsilon} \sum_{i \leq m} A\left(\ell_{i}\right)^{2} r\|k\|_{\infty, 2, r} \leq N^{2 \varepsilon}\left[1+\left(\sqrt{N} \ell_{m}\right)^{-1}\right]^{2} r\|k\|_{\infty, 2, r} .
$$

From the definition of $h$ in Lemma A.4, we need to consider two contributions of $h$ : one is $k=\bar{\partial} f$, the other involves the $s$ integration. Since $f$ is supported in a ball of radius $b$, the contribution from $\bar{\partial} f$ can be trivially bounded by

$$
N^{2 \varepsilon}\left[1+\left(\sqrt{N} \ell_{m}\right)^{-1}\right]^{2} b\|h\|_{\infty, 2, b} \leq N^{2 \varepsilon}\left[1+\left(\sqrt{N} \ell_{m}\right)^{-1}\right]^{2}\|f\|_{\infty, 3, b}
$$

where we have replaced $r$ in (A.24) by $b$.

Applying now A.24 to $k=\vec{\partial} f_{s}$, we bound the other term of $h$ involving $s$ integration by

$$
\begin{aligned}
& N^{2 \varepsilon}\left[1+\left(\sqrt{N} \ell_{m}\right)^{-1}\right]^{2} \ell^{-2} \int_{0}^{\log N} \frac{\mathrm{d} s}{s} b_{s}\left\|\partial f_{s}\right\|_{\infty, 2, b_{s}}+N^{-A}\|f\|_{\infty, 1, b} \\
& \leq N^{2 \varepsilon}\left[1+\left(\sqrt{N} \ell_{m}\right)^{-1}\right]^{2} \ell^{-2} \int_{0}^{\log N} \frac{\mathrm{d} s}{s}(b \wedge s)^{2}\|f\|_{\infty, 3, b}+N^{-A}\|f\|_{\infty, 1, b} \\
& \leq N^{2 \varepsilon}\left[1+\left(\sqrt{N} \ell_{m}\right)^{-1}\right]^{2} \frac{b^{2}}{\ell^{2}}\|f\|_{\infty, 3, b}+N^{-A}\|f\|_{\infty, 1, b},
\end{aligned}
$$


where we have again used A.14.

Combining Case 1 and 2, we have bounded (A.16) by

$$
N^{2 \varepsilon}\left[\left(1+\left(\sqrt{N} \ell_{m}\right)^{-1}\right)^{2}+N \ell_{m}^{2}\right]\left[1+\frac{b^{2}}{\ell^{2}}\right]\|f\|_{\infty, 3, b}+N^{-A}\|f\|_{\infty, 3, b},
$$

where the term $N \ell_{m}^{2}\left[1+\frac{b^{2}}{\ell^{2}}\right]$ comes from the Case 1 and the other terms come from Case 2 . Recalling $\ell_{m}=N^{-1 / 2+\varepsilon}$, we have proved A.16.

Proof of Theorem 2.4. We will assume $V=0$; the general case can be proved in a similar way. We again employ the loop equation and calculate

$$
\begin{aligned}
& \frac{1}{\beta} \log \mathbb{E}_{0}^{U^{\ell}} \mathrm{e}^{-t \beta X_{0}^{f}}=\frac{1}{\beta} \int_{0}^{t} \mathrm{~d} s \frac{\partial}{\partial s} \log \mathbb{E}_{0}^{U^{\ell}} \mathrm{e}^{-s \beta X_{0}^{f}}=\int_{0}^{t} \mathrm{~d} s\left(-\mathbb{E}_{s f / N}^{U^{\ell}} X_{s f / N}^{f}+N \int f\left(\mu_{0}-\mu_{s f / N}\right)\right) \\
& =\int_{0}^{t} \mathrm{~d} s \mathbb{E}_{s f / N}^{U^{\ell}}\left(\frac{1}{N} W_{s f / N}^{h_{s}}-\frac{1}{N \beta} \sum_{j} \partial h_{s}\left(z_{j}\right)\right. \\
& \left.-N \iint\left(h_{s}(z)-h_{s}(w)\right) \partial U^{\ell}(z-w) \tilde{\mu}_{s f / N}(\mathrm{~d} z) \tilde{\mu}_{s f / N}(\mathrm{~d} w)+N \int f\left(\mu_{0}-\mu_{s f / N}\right)\right),
\end{aligned}
$$

where $h_{s}(z)=\frac{1}{\pi \rho_{s f / N}(z)} \bar{\partial}\left(1-m^{2} \Delta^{-1}\right) f(z)$ and $\mu_{s f / N}$ as in A.2. By Lemma A.5.

$$
N \mathbb{E}_{s f / N}^{U^{\ell}} \iint\left(h_{s}(z)-h_{s}(w)\right) \partial U^{\ell}(z-w) \tilde{\mu}_{s f / N}(\mathrm{~d} z) \tilde{\mu}_{s f / N}(\mathrm{~d} w)=\mathrm{O}\left(N^{\varepsilon}\left(1+\frac{b^{2}}{\ell^{2}}\right)\right)\|f\|_{\infty, 3, b} .
$$

By Lemma 8.3. $\mathbb{E}_{s f / N}^{U^{\ell}} W_{s f / N}^{h_{s}}=0$. Using $m=1 / \ell$, we have

$$
\begin{aligned}
\int_{0}^{t} \mathrm{~d} s N \int f\left(\mu_{0}-\mu_{s f / N}\right)= & -N \int_{0}^{t} \mathrm{~d} s \int f(z)\left[\frac{s}{4 \pi N}\left(\Delta-m^{2}\right) f(z) m(\mathrm{~d} z)+\frac{m^{2}}{2 \pi}\left(c_{0}-c_{s f / N}\right)\right] \\
& =\mathrm{O}\left(\int_{0}^{t} \mathrm{~d} s \frac{s}{4 \pi} \int f\left(-\Delta+m^{2}\right) f\right)=\mathrm{O}\left(t^{2}\left(1+b^{2} \ell^{-2}\right)\right)\|f\|_{\infty, 2, b}^{2},
\end{aligned}
$$

where we have used $\left(c_{0}-c_{s f / N}\right)=\frac{s}{2 N} \int f(z) m(\mathrm{~d} z)$ which is a consequence of 2.14 and the normalization condition $\int \mu_{V}^{\ell}=1$. Finally, using the fact that the local density is bounded and A.14, we have

$$
\int_{0}^{t} \mathrm{~d} s \frac{1}{N \beta} \mathbb{E}_{s f / N}^{U^{\ell}} \sum_{j} \partial h_{s}\left(z_{j}\right)=\mathrm{O}(t)\left\|\partial \rho_{s f / N}^{-1} \bar{\partial}\left(f-m^{2} \Delta^{-1}\right) f\right\|_{1}=\mathrm{O}(t)\left(1+\frac{b^{2}}{\ell^{2}}\right) b^{2}\|f\|_{\infty, 2, b} .
$$

Collecting these estimates gives

$$
\frac{1}{\beta} \log \mathbb{E}_{0}^{U^{\ell}} \mathrm{e}^{-t \beta X_{0}^{f}}=\mathrm{O}\left(N^{\varepsilon}\left(1+\frac{b^{2}}{\ell^{2}}\right)\right)\left[t\|f\|_{\infty, 3, b}+t^{2}\|f\|_{\infty, 3, b}^{2}\right]
$$

By Markov's inequality with $t=1 /\|f\|_{\infty, 3, b}$ this implies $X_{0}^{f}=\mathrm{O}\left(N^{\varepsilon}\right)\left(1+b^{2} / \ell^{2}\right)\|f\|_{\infty, 3, b}$ with probability at least $1-\mathrm{e}^{-N^{\varepsilon}}$, which proves Theorem 2.4 .

With Theorem 2.4 proved, we can now prove Proposition 2.5. 
Proof of Proposition 2.5. We apply the identity (A.9) which expands the left hand side of 2.25) into a Taylor series with error term. To prove (2.25), we only have to estimate each term in the summation in A.8. From the rigidity estimate Theorem 2.4. we have

$$
N \int(x-z)^{k_{1}}(\bar{x}-\bar{z})^{k_{2}} \omega_{s}(z-x) \tilde{\mu}(\mathrm{d} z)=\mathrm{O}\left(N^{\varepsilon}\right) s^{k_{1}+k_{2}-2}\left(1+\frac{s}{\ell}\right)^{2},
$$

and a similar estimate holds around $y$. These two bounds together imply that each term in the summation in A.8 is bounded by $\mathrm{O}\left(N^{\varepsilon}\right)\left(\frac{1}{s^{4}}+\frac{1}{\ell^{4}}\right) s^{j}\left\|\nabla^{j} g\right\|_{1}$ and this completes the proof of the proposition.

A.4. Proof of Lemma 8.11. In this subsection, we prove Lemma 8.11 which is an estimate with respect to the Coulomb gas with an angle correction term. Recall the definition of the long range interaction in (8.11), given by

$$
A_{V}^{h,+}=\frac{N}{2} \iint_{z \neq w} \Psi_{h}^{+}(z, w) \tilde{\mu}_{V}(\mathrm{~d} z) \tilde{\mu}_{V}(\mathrm{~d} w),
$$

where

$\Psi_{h}^{+}(z, w)=\Phi_{\theta}^{+}(z-w)(\bar{z}-\bar{w})(h(z)-h(w)), \quad \Phi_{\theta}^{+}(z-w)=\int_{\theta}^{\infty} \Phi(z-w, r) \frac{\mathrm{d} r}{r^{5}}=\frac{1-\mathrm{e}^{-\frac{|z-w|^{2}}{2 \theta^{2}}}}{|z-w|^{2}}$.

Our proof of Lemma 8.11 is based on transporting a proof for the Yukawa gas to the Coulomb setting. For this purpose, recall that the long range part in the decomposition (A.17) for the Yukawa gas is of the following form

$$
G_{\sigma}(z, w)=(h(z)-h(w)) \sum_{i \leq m} U_{i}(z-w)
$$

with $\ell_{m} \sim N^{-1 / 2+\sigma}$ for some $\sigma>0$ fixed. Using the rigidity estimate 2.23) for the Yukawa gas, we claim that

$$
\left|\mathbb{E}_{V}^{U^{\ell}}\left(N \iint_{z \neq w} G_{\sigma}(z, w) \tilde{\mu}_{V}(\mathrm{~d} z) \tilde{\mu}_{V}(\mathrm{~d} w)\right)\right| \leqslant N^{-\sigma+\varepsilon} r\|h\|_{\infty, 2, r} .
$$

To prove this bound, we keep the estimate (A.21) unchanged but for A.22), instead of the local law, we apply the rigidity estimate 2.23

$$
\left|\int \omega_{q}(w-y) \tilde{\mu}(\mathrm{d} w)\right| \leq N^{-1+\varepsilon}\left(1+q^{2} / \ell^{2}\right)\left\|\omega_{q}(\cdot-y)\right\|_{\infty, 3, q} .
$$

Using $\left\|\omega_{q}(\cdot-y)\right\|_{\infty, 3, q} \leq q^{-2}$, we therefore improved A.24 to

$$
\left|\sum_{i \leq m} N \iint_{z \neq w} M_{i}(z, w) \tilde{\mu}_{V}(\mathrm{~d} z) \tilde{\mu}_{V}(\mathrm{~d} w)\right| \leqslant \frac{N^{2 \varepsilon}}{\sqrt{N} \ell_{m}}\left(1+\frac{1}{\sqrt{N} \ell_{m}}\right)^{2} r\|h\|_{\infty, 2, r},
$$

gaining a factor $\left(\sqrt{N} \ell_{m}\right)^{-1}$ over A.24). This proves A.29). Notice that the extra derivative required in applying the rigidity estimate is performed on the test function $\omega$, so the number of derivatives required on $h$ remains the same when compared with the earlier results relying on the local law. 
We return to estimating $A^{+}$, ie.e the proof of Lemma 8.11 for Coulomb gases with or without an angle correction term. Notice that $\Psi_{h}^{+}(z, w)$ is of the form $k(z-w)(h(z)-h(w))$ with $k(z-w)=\Phi_{\theta}^{+}(z-w)(\bar{z}-\bar{w})$. Hence we can apply the decomposition (A.17) and express $A^{+}$ similarly to $G$ in A.28). Due to the short range cutoff by $\theta$ in the definition of $\Phi_{\theta}^{+}$, we effectively have a cutoff at the scale $\theta=N^{-1 / 2+\sigma}$. This is consistent with the choice of $\ell_{m} \sim N^{-1 / 2+\sigma}$ in A.28). Instead of the rigidity estimate (2.23) for the Yukawa gas, we apply the one with respect to the Coulomb gas with an angle correction term, i.e., 8.40). Notice that in 8.40, $\left\|\omega_{q}(\cdot-y)\right\|_{\infty, 3, q}$ in A.30 was replaced by $\left\|\omega_{q}(\cdot-y)\right\|_{\infty, 4, q}$. Since $\omega$ is a smooth mollifier, both norms are of the same order. Following the argument in the proof of (A.29), we have therefore proved Lemma 8.11 .

The key observation in this proof is that the application of the rigidity estimate yields an improvement over the local law for all functions of scales bigger than $N^{-1 / 2+\varepsilon}$. So any estimates based on the local laws can be improved by a factor $N^{-\sigma}$ for functions at scales greater than $N^{-1 / 2+\sigma}$.

\section{Appendix B Local law for the Yukawa and Coulomb gas}

In this appendix, we prove Theorems 2.22 .3 . Our presentation follows closely that of 6 and we therefore mainly present the differences. The interaction in Theorem 2.2 is a Yukawa potential instead of the Coulomb potential in [6. Theorem 1.1]. To allow for this change, we first develop generalizations of the basic potential estimates used in [6] to the Yukawa potential. Once these estimates are given, the rest of the proof is parallel to that of [6. Theorem 1.1]. The proof of Theorem 2.3 is essentially the as same as that of [6, Theorem 1.1] under slightly generalized assumptions. Its proof requires only minor adjustment to the original proof which we will comment on later in this appendix.

B.1. Some potential theory for the Yukawa potential. We start with properties of the Yukawa potential. They are parallel to those of the Coulomb potential used in [6].

The following proposition characterizes the Yukawa potential of the equilibrium measure in terms of an obstacle problem. The proposition is similar to the analogous result for the Coulomb case, but requires a slightly different characterization of the admissible potentials than the one stated for the Coulomb case in [24], for example. We give a proof for completeness, as we were unable to locate a suitable reference.

Proposition B.1. Under the assumptions of Theorem 2.1, the following holds. Define

$$
u_{V, \ell}(z)=\sup _{\nu, c}\left\{-U_{\nu}^{\ell}(z)+c:-U_{\nu}^{\ell}+c \leqslant \frac{1}{2} V, \nu \geqslant 0, \nu(\mathbb{C}) \leqslant 1\right\}
$$

where the supremum is over measures $\nu$ and constants $c$. Then $u_{V, \ell}=-U_{\mu_{V}}^{\ell}+c_{V}$ where $c_{V}$ is the constant in 2.13.

Proof. By definition, $u_{V, \ell} \geqslant-U_{\mu_{V}}^{\ell}+c_{V}$ since the right-hand side is a subsolution of the same form as inside the supremum in (B.1). To prove that in fact equality holds, suppose otherwise that $u_{V, \ell}\left(z_{0}\right)>-U_{\mu_{V}}^{\ell}\left(z_{0}\right)+c_{V}$ for some $z_{0} \in \mathbb{C}$. Then there exists some positive measure $\tilde{\eta}$ with $\tilde{\eta}(\mathbb{C}) \leqslant 1$ and constant $c \in \mathbb{R}$ for which $-U_{\tilde{\eta}}^{\ell}\left(z_{0}\right)+c>-U_{\mu_{V}}^{\ell}\left(z_{0}\right)+c_{V}$. By considering $\left.\tilde{\eta}\right|_{B_{R}}$ for $R>0$ large enough we may suppose that $\tilde{\eta}$ is compactly supported, and by convolving with a smooth mollifier we may suppose $\tilde{\eta}$ has a smooth density. Consider the function

$$
g(z)=\max \left(-U_{\tilde{\eta}}^{\ell}(z)+\tilde{c},-U_{\mu_{V}}^{\ell}(z)+c_{V}\right) .
$$


By writing $\max (a, b)=\frac{a+b}{2}+\frac{|a-b|}{2}$ and convolving the absolute value by a smooth, compactly supported, symmetric mollifier, we may check that $g(z)=-U_{\eta}^{\ell}(z)+c$ for some positive measure $\eta$, and necessarily $c=\max \left(\tilde{c}, c_{V}\right)$. To show that $g$ is a subsolution of the form in (B.1) we need to show that $\eta(\mathbb{C}) \leqslant 1$. For this, suppose without loss of generality that $c=\tilde{c}$. Denote $D=\left\{z:-U_{\tilde{\eta}}^{\ell}(z)+\tilde{c}<-U_{\mu_{V}}^{\ell}(z)+c_{V}\right\}$. Then

$$
\begin{aligned}
& \eta(\partial D)=\int_{\partial D} \partial_{n}\left(-U_{\tilde{\eta}}^{\ell}-\left(-U_{\mu_{V}}^{\ell}\right)\right)=\int_{D} \Delta\left(-U_{\tilde{\eta}}^{\ell}-\left(-U_{\mu_{V}}^{\ell}\right)\right) \\
& =\int_{D}\left(\tilde{\eta}-\mu_{V}\right)+m^{2} \int_{D}\left(-U_{\tilde{\eta}}^{\ell}-\left(-U_{\mu_{V}}^{\ell}\right)\right) \\
& =\int_{D}\left(\tilde{\eta}-\mu_{V}\right)+m^{2} \int_{D}\left(-U_{\tilde{\eta}}^{\ell}+\tilde{c}-\left(-U_{\mu_{V}}^{\ell}+c_{V}\right)\right)+m^{2} \int_{D}\left(c_{V}-\tilde{c}\right) \leqslant \tilde{\eta}(D)-\mu_{V}(D) .
\end{aligned}
$$

Thus $\eta(D \cup \partial D)=\eta(\partial D)+\mu_{V}(D) \leqslant \tilde{\eta}(D)$. Since clearly $\eta(\mathbb{C} \backslash(\partial D \cup D))=\tilde{\eta}(\mathbb{C} \backslash(\partial D \cup D))$, we have $\eta(\mathbb{C}) \leqslant \tilde{\eta}(C) \leqslant 1$. Now,

$$
\begin{gathered}
g-\left(-U_{\mu_{V}}^{\ell}+c_{V}\right) \geqslant 0, \\
\left(\Delta-m^{2}\right)\left(-U_{\eta}^{\ell}-\left(-U_{\mu_{V}}^{\ell}\right)=\eta-\mu \geqslant m^{2}\left(c-c_{V}\right) \geqslant 0 .\right.
\end{gathered}
$$

Since strict inequality holds in the first inequality for $z_{0}$ and the functions involved are continuous, equality (as distributions) cannot hold on the second line. But this implies $\eta(\mathbb{C})>\mu_{V}(\mathbb{C})=$ 1 , a contradiction.

We also require the following properties of the Yukawa potential 2.1). Recall that

$$
Y^{\ell}(z)=g(a), \quad a=\frac{|z|}{2 \ell}, \quad \text { where } g(a)=\int_{1}^{\infty} \mathrm{e}^{-a(s+1 / s)} \frac{\mathrm{d} s}{s} .
$$

In fact, $g(a)=K_{0}(2 a)$ where $K_{0}$ is a modified Bessel function of the second kind. In particular, the gradient of the Yukawa potential has the expression:

$$
\nabla Y^{\ell}(z)=g^{\prime}\left(\frac{|z|}{2 \ell}\right) \frac{\nabla|z|}{2 \ell}=g^{\prime}\left(\frac{|z|}{2 \ell}\right) \frac{|z|}{2 \ell} \frac{1}{\bar{z}}=-\frac{1}{\bar{z}} f(a), \quad a=\frac{|z|}{2 \ell},
$$

where

$$
f(a)=\int_{1}^{\infty} a(s+1 / s) \mathrm{e}^{-a(s+1 / s)} \frac{\mathrm{d} s}{s}=\int_{a}^{\infty}\left(1+a^{2} / s^{2}\right) \mathrm{e}^{-\left(s+a^{2} / s\right)} \mathrm{d} s .
$$

The function $f$ is smooth in $a>0$, satisfies $f(0)=1$, and is positive and decreasing. As a consequence we have $\left|\nabla Y^{\ell}(2 r)\right| \leqslant\left|\nabla Y^{\ell}(r)\right| / 2$.

Since $\nabla Y^{\ell}(z) \sim \nabla \log \frac{1}{|z|}$ for $z \rightarrow 0$, the following formula (B.4) follows as in [6, (3.21)]. Let $\gamma \subset \mathbb{C}$ be a $C^{1}$ curve and $\eta$ a measure supported on $\gamma$ for which the potential $U_{\eta}^{\ell}$ is continuous on $\mathbb{C}$. Then for $z \in \gamma$ we have

$$
\partial_{n}^{-} U_{\eta}^{\ell}(z)=\pi \lim _{r \rightarrow 0^{+}} \frac{\eta\left(B_{r}(z)\right)}{s\left(B_{r}(z)\right)}+\int_{\gamma} \nabla Y^{\ell}(z-w) \cdot \bar{n} \eta(d w),
$$

where $\partial_{n}^{-}$denotes a one-sided derivative in the normal direction $\bar{n}=\bar{n}(z)$ and $s$ denotes the arclength measure of $\gamma$, if the limit on the right-hand side exists.

The formula (B.4) implies the following estimate for the density of a measure supported on $\partial \mathbb{D}$. For the statement, define

$$
I^{\ell}:=\frac{1}{2 \pi} \int_{\partial \mathbb{D}} f\left(\frac{|1-w|}{2 \ell}\right) s(\mathrm{~d} w) \in(0,1)
$$


and note that $I^{\ell}$ is increasing in $\ell$ with $I^{\ell}=1+O(1 / \ell)$ as $\ell \rightarrow \infty$ and $I^{\ell}=O(\ell)$ as $\ell \rightarrow 0$. The proofs of the following Lemma B.2 and B.3 are based on elementary potential theory.

Lemma B.2. For any (signed) measure $\omega$ supported on $\partial \mathbb{D}$, denote by $\bar{\omega}=\frac{1}{2 \pi} \int \mathrm{d} \omega$ the constant part of $\omega$. Then

$$
\begin{aligned}
\left\|\frac{d \omega}{d s}-\bar{\omega}\right\|_{\infty} & \leqslant \frac{2}{\pi I^{\ell}}\left\|\partial_{n}^{-} U_{\omega}^{\ell}\right\|_{\infty, \partial \mathbb{D}} \\
\left\|\frac{d \omega}{d s}\right\|_{\infty} & \leqslant \frac{1}{\pi\left(1-I^{\ell}\right)}\left\|\partial_{n}^{-} U_{\omega}^{\ell}\right\|_{\infty, \partial \mathbb{D}}
\end{aligned}
$$

and

$$
\partial_{n}^{-} U_{\bar{\omega}}^{\ell}(1)=\frac{1}{2 \pi} \int_{\partial \mathbb{D}} \partial_{n}^{-} U_{\omega}^{\ell}(z) s(\mathrm{~d} z) \leqslant\left\|\partial_{n}^{-} U_{\omega}^{\ell}\right\|_{\infty, \partial \mathbb{D}} .
$$

Proof. By (B.4), we have

$$
\frac{d \omega}{d s}(z)=\frac{1}{2 \pi}\left(2 \partial_{n}^{-} U_{\omega}^{\ell}(z)-2 \int \nabla Y^{\ell}(z-w) \cdot \bar{n}(z) \omega(\mathrm{d} w)\right) .
$$

For $z, w$ with $|z|=|w|=1$ and $z \neq w$,

$$
\frac{z-w}{|z-w|^{2}} \cdot \frac{z}{|z|}=\operatorname{Re}\left(\frac{z-w}{|z-w|^{2}} \bar{z}\right)=\operatorname{Re}\left(\frac{1-w / z}{|1-w / z|^{2}}\right)=\frac{1}{2},
$$

and, by (B.3), therefore

$$
-2 \nabla Y^{\ell}(z-w) \cdot n(z)=f\left(\frac{|z-w|}{2 \ell}\right) .
$$

It follows that

$$
\frac{d \omega}{d s}(z)=\frac{1}{2 \pi}\left(2 \partial_{n}^{-} U_{\omega}^{\ell}(z)+\int f\left(\frac{|z-w|}{2 \ell}\right) \omega(\mathrm{d} w)\right) .
$$

Integrating $(\overline{\mathrm{B} .12})$, we obtain the identity

$$
\left(1-I^{\ell}\right) \int \omega=\frac{2}{2 \pi} \int_{\partial \mathbb{D}} \partial_{n}^{-} U_{\omega}^{\ell}(z) s(\mathrm{~d} z) .
$$

Applying this identity to $\bar{\omega}$, since $\int \mathrm{d} \omega=\int \mathrm{d} \bar{\omega}$, we obtain

$$
\partial_{n}^{-} U_{\bar{\omega}}^{\ell}(1)=\frac{1}{2 \pi} \int_{\partial \mathbb{D}} \partial_{n}^{-} U_{\bar{\omega}}^{\ell}(z) s(\mathrm{~d} z)=\frac{1}{2 \pi} \int_{\partial \mathbb{D}} \partial_{n}^{-} U_{\omega}^{\ell}(z) s(\mathrm{~d} z) .
$$

This shows (B.8). Similarly, from (B.12), we obtain

$$
\left(1-I^{\ell}\right)\left\|\frac{d \omega}{d s}\right\|_{\infty} \leqslant \frac{2}{2 \pi}\left\|\partial_{n}^{-} U_{\omega}^{\ell}\right\|_{\infty}
$$

which shows (B.7), and also similarly,

$$
\left\|\frac{d \omega}{d s}-\frac{1}{2 \pi} \int f\left(\frac{|\cdot-w|}{2 \ell}\right) \omega(\mathrm{d} w)\right\|_{\infty} \leqslant \frac{2}{2 \pi}\left\|\partial_{n}^{-} U_{\omega}^{\ell}\right\|_{\infty} .
$$

To show (B.6), i.e.,

$$
I^{\ell}\left\|\frac{d \omega}{d s}-\frac{1}{2 \pi} \int d \omega\right\|_{\infty} \leqslant \frac{4}{2 \pi}\left\|\partial_{n}^{-} U_{\omega}^{\ell}\right\|_{\infty}
$$


write

$$
\begin{aligned}
& \frac{d \omega}{d s}-\frac{1}{2 \pi} \int f\left(\frac{|\cdot-w|}{2 \ell}\right) \omega(d w) \\
& =\frac{d \omega}{d s}-\frac{1}{2 \pi} \int d \omega+\frac{1}{2 \pi} \int\left(1-f\left(\frac{|\cdot-w|}{2 \ell}\right)\right) \omega(d w) \\
& =\frac{d \omega}{d s}-\frac{1}{2 \pi} \int d \omega+\frac{1}{2 \pi} \int\left(1-f\left(\frac{|\cdot-w|}{2 \ell}\right)\right)\left(\frac{d \omega}{d s}(w)-\frac{1}{2 \pi} \int d \omega\right) s(d w) \\
& \quad+\frac{1}{2 \pi} \int\left(1-f\left(\frac{|\cdot-w|}{2 \ell}\right)\right) s(d w) \cdot \frac{1}{2 \pi} \int d \omega .
\end{aligned}
$$

Taking absolute values on the supremum over $\partial \mathbb{D}$, and using (B.15), therefore

$$
\begin{aligned}
& \left\|\frac{d \omega}{d s}-\frac{1}{2 \pi} \int f\left(\frac{|\cdot-w|}{2 \ell}\right) \omega(d w)\right\|_{\infty} \\
& \geqslant\left\|\frac{d \omega}{d s}-\frac{1}{2 \pi} \int d \omega\right\|_{\infty}-\left(1-I^{\ell}\right)\left\|\frac{d \omega}{d s}-\frac{1}{2 \pi} \int d \omega\right\|_{\infty}-\left(1-I^{\ell}\right)\left|\frac{1}{2 \pi} \int d \omega\right|, \\
& \geqslant I^{\ell}\left\|\frac{d \omega}{d s}-\frac{1}{2 \pi} \int d \omega\right\|_{\infty}-\frac{2}{2 \pi}\left\|\partial_{n}^{-} U_{\omega}^{\ell}\right\|_{\infty} .
\end{aligned}
$$

Together with (B.16), we obtain (B.17).

We will also need the following properties of the function

$$
l_{r}(z)=\left(Y^{\ell} * \frac{1}{\pi r^{2}} 1_{B(0, r)}\right)(z)
$$

Clearly, $l_{r}(z)$ is radial, so we can define $h_{r}$ through $\nabla l_{r}(z)=-(z /|z|) h_{r}(|z|)$ for $z \neq 0$.

Lemma B.3. For any $\ell>0$, the function $h_{r}(t)$ is positive, increasing for $t \leqslant r$, decreasing for $t \geqslant r$, and

$$
h_{r}(t) \geqslant\left|\nabla Y^{\ell}(t)\right| \quad \text { for } t \geqslant r .
$$

Proof. That $h_{r}(t)$ is increasing for $t<r$ can be seen as follows. For $t>0$, since $Y^{\ell}$ is symmetric,

$$
\nabla l_{r}(t)=\int_{|z| \leqslant r} \nabla Y^{\ell}(z-t) m(\mathrm{~d} z)=\int_{U_{r}(t)} \operatorname{Re} \nabla Y^{\ell}(z-t) m(\mathrm{~d} z)=\int_{U_{r}(t)-t} \operatorname{Re} \nabla Y^{\ell}(z) m(\mathrm{~d} z)
$$

where $U_{r}(t)$ is $\{|z| \leqslant r\}$ minus the region $\{|z| \leqslant r: \operatorname{Re} z>t\}$ and the reflection of the latter region about the axis $\operatorname{Re} z=t$. In particular, the region $U_{r}(t)-t$ is increasing in $t$.

To prove (B.19) and that $h_{r}(t)$ is decreasing for $t>r$, we use the Yukawa version of Newton's shell theorem: there is $M^{\ell}(r) \geqslant 1$ such that for $t \geqslant r$,

$$
\frac{1}{2 \pi r} \int_{|z|=r} Y^{\ell}(t-z) s(\mathrm{~d} z)=M^{\ell}(r) Y^{\ell}(t)
$$

Denote the left-hand side by $f(t)$. Then $f$ is a bounded and radially symmetric solution to $\left(-\Delta+1 / \ell^{2}\right) f(z)=0$ for $|z|>r$. Therefore, for $t>r$,

$$
f^{\prime \prime}(t)+\frac{1}{t} f^{\prime}(t)-\frac{1}{\ell^{2}} f(t)=0,
$$


and the solutions to this ODE are of the form

$$
f(t)=A I_{0}(t / \ell)+B K_{0}(t / \ell),
$$

where the $I_{n}$ are the modified Bessel functions of the first kind and the $K_{n}$ are the modified Bessel functions of the second kind, and $A, B$ are constants depending on $r$. The Yukawa potential equals $Y^{\ell}(z)=K_{0}(|z| / \ell)$. Since $I_{0}(t) \rightarrow \infty$ as $t \rightarrow \infty$, therefore $A=0$ and thus $f(t)=B K_{0}(t / \ell)=B Y^{\ell}(t)$ for some constant $B=M^{\ell}(r)$.

To see that $B \geqslant 1$, we assume that $r=1$ and $\ell=1 / 2$ to simplify the notation (the general case is analogous). Denote by $\theta$ the angle of $z$ with respect to the real axis so that $|t-z|^{2}=t^{2}-2 t \cos \theta+1$. Recall (2.1) and note that the function $\tilde{g}(x)=\int_{1}^{\infty} \mathrm{e}^{-\sqrt{x}(s+1 / s)} \frac{\mathrm{d} s}{s}$ is convex for $x \geqslant 1$. With $x=t^{2}-2 t \cos \theta+1$ and using the Jensen inequality, we have

$$
f(t)=\mathbb{E} \tilde{g}\left(t^{2}-2 t \cos \theta+1\right) \geq \tilde{g}\left(t^{2}-2 t \mathbb{E} \cos \theta+1\right)=\tilde{g}\left(t^{2}+1\right), \quad \mathbb{E}=(2 \pi)^{-1} \int \mathrm{d} \theta .
$$

It is elementary to check that

$$
\lim _{t \rightarrow \infty} \frac{\tilde{g}\left(t^{2}+1\right)}{\tilde{g}\left(t^{2}\right)}=1
$$

Hence we have proved that $B \geq 1$. (In fact, $B>1$ for any $r, \ell$ fixed, but we will not need this.)

In particular, for $t \geqslant r$,

$$
l_{r}(t)=\frac{1}{\pi r^{2}} \int_{|z| \leqslant r} Y^{\ell}(z-t)=\frac{1}{\pi r^{2}} \int_{0}^{r} \int_{|z|=s} Y^{\ell}(z-t) s(\mathrm{~d} z) \mathrm{d} r=\tilde{M}^{\ell}(t) Y^{\ell}(t)
$$

with $\tilde{M}^{\ell}(t)=\frac{1}{\pi r^{2}} \int_{0}^{r}(2 \pi r) M^{\ell}(r) \mathrm{d} r \geqslant 1$. Thus, for $t \geqslant r$,

$$
\left|\nabla l_{r}(t)\right|=\tilde{M}^{\ell}(r)\left|\nabla Y^{\ell}(t)\right| \geqslant\left|\nabla Y^{\ell}(t)\right| \text {. }
$$

The first equality implies that $\left|\nabla l_{r}(t)\right|$ is decreasing for $t>r$ since $\left|\nabla Y^{\ell}(t)\right|$ is decreasing. The inequality implies that $(\mathrm{B} .19)$ holds.

In Section B.2 below, we require the following two technical lemmas to locate the bulk of the support of a perturbed equilibrium measure. Lemma B.4 is a small adaption of [6, Lemma 3.6] to the Yukawa case; Lemma B.5 is a similar statement that applies to a radially symmetric potential on the boundary of a disk instead of a point charge outside a disk.

Lemma B.4. For any $z_{0} \in \mathbb{C}, w \in \mathbb{C}, \sigma>\frac{1}{2}$, and $r \in(0,1)$ such that that $\left|z_{0}-w\right| \geqslant 2 r$, there exist $\tilde{z} \in \mathbb{C}$ and $k \in \mathbb{R}$ such that

$$
\sigma\left(l_{r}\left(z_{0}-\tilde{z}\right)+k\right)=\frac{1}{2} Y^{\ell}\left(z_{0}-w\right) \quad \text { and } \quad \sigma\left(l_{r}(z-\tilde{z})+k\right) \leqslant \frac{1}{2} Y^{\ell}(z-w) \text { for all } z \in \mathbb{C} .
$$

Moreover, the point $\tilde{z}$ lies on the line passing through $z_{0}$ and $w$ at distance at most $r$ from $z_{0}$ between $z_{0}$ and $w$.

Proof. By (B.19) and since $\sigma \geqslant \frac{1}{2}$, the map $z \mapsto \sigma \nabla l_{r}\left(z_{0}-z\right)$ takes $B_{r}\left(z_{0}\right)$ onto $B_{\sigma\left|\nabla l_{r}(r)\right|}(0) \supset$ $B_{\sigma\left|\nabla Y^{\ell}(r)\right|} \supset B_{\left|\nabla Y^{\ell}(2 r)\right|}(0)$, where we also used $\left|\nabla Y^{\ell}(2 r)\right| \leqslant \frac{1}{2}\left|\nabla Y^{\ell}(r)\right|$. Therefore, as in 6 , Lemma 3.6], it follows there exists a unique choice of $\tilde{z} \in B_{r}\left(z_{0}\right)$ so that the gradients of $\sigma l_{r}(\cdot-\tilde{z})$ and $\frac{1}{2} Y^{\ell}(\cdot-w)$ match at $z_{0}$. By choice of $k$, we can in addition arrange

$$
\sigma\left(l_{r}\left(z_{0}-\tilde{z}\right)+k\right)=\frac{1}{2} Y^{\ell}\left(z_{0}-w\right) .
$$


It remains to be shown that with the above choice it is in fact true that

$$
\sigma\left(l_{r}(z-\tilde{z})+k\right) \leqslant \frac{1}{2} Y^{\ell}(z-w) \quad \text { for all } z \in \mathbb{C} .
$$

As in the Coulomb case, the point must $\tilde{z}$ lie on the line between the points $z_{0}$ and $w$, and it suffices to show the inequality on this line (by the same argument as in the Coulomb case, 6 Lemma 3.6]). Moreover, without loss of generality, we can assume that $w=0, z_{0}>0, \tilde{z}>0$, so that this line is $\mathbb{R}$. Thus it needs to be shown that

$$
f(x):=\frac{1}{2} Y^{\ell}(x) \geqslant \sigma\left(l_{r}(x-\tilde{z})+k\right)=: g(x), \quad x \in \mathbb{R} .
$$

As in the Coulomb case, denote by $h$ the common tangent of the graphs of $f$ and $g$ drawn at $x=z_{0}$. Since $f$ is convex and $g$ is concave on $[\tilde{z}-r, \tilde{z}+r]$, the graph of $f$ lies above $h$ and the graph of $g$ lies below $h$ on this interval. Especially $g(x) \leqslant f(x)$ on $[\tilde{z}-r, \tilde{z}+r]$. Moreover, since $f^{\prime}(x)<0$ and $g^{\prime}(x)>0$ for $x \in(0, \tilde{z})$, the inequality $g(x) \leqslant f(x)$ holds by these observations for $x \in(0, \tilde{z}+r]$.

To prove the inequality for $x \in[\tilde{z}+r, \infty)$, we have $g^{\prime}(t) \leqslant f^{\prime}(t+\tilde{z}) \leqslant f^{\prime}(t)$ by $(\overline{\mathrm{B} .19})$, for $t \in[\tilde{z}+r, \infty)$. It follows that

$$
g(x)-g(\tilde{z}+r)=\int_{\tilde{z}+r}^{x} g^{\prime}(t) d t \leqslant \int_{\tilde{z}+r}^{x} f^{\prime}(t) d t=f(x)-f(\tilde{z}+r),
$$

which by $g(\tilde{z}+r) \leqslant f(\tilde{z}+r)$ implies the desired inequality $g(x) \leqslant f(x)$, now proven for $x \in(0, \infty)$. The case $x<0$ is actually not required for the application, but true. Indeed, for $x \in(-\infty, 0)$ it also holds that $g^{\prime}(x) \leqslant f^{\prime}(x)$ and it is clear that $f(x) \geqslant g(x)$ as $x \rightarrow 0^{-}$, so it remains to check the inequality as $x \rightarrow-\infty$. As in the Coulomb case, this follows from $k<0$, which follows from

$$
\sigma k=\frac{1}{2} Y^{\ell}\left(z_{0}\right)-\sigma l_{r}\left(z_{0}-\tilde{z}\right)<\frac{1}{2} Y^{\ell}(2 r)-\sigma l_{r}(r)<0 .
$$

This completes the proof.

Lemma B.5. Let $r \in\left(0, \frac{1}{2}\right)$ and $\sigma \geqslant \sigma_{0}$ and $\ell \geqslant \ell_{0}$, where $\sigma_{0}$ and $\ell_{0}$ are sufficiently large absolute constants. Then for any $z_{0} \in \mathbb{C}$ with $\left|z_{0}\right|<1-2 r$, there exists a constant $k \in \mathbb{R}$ and $\tilde{z} \in \mathbb{C}$ with $|\tilde{z}|<1-r$ on the line through 0 and $z_{0}$ such that

$$
\sigma\left(l_{r}\left(z_{0}-\tilde{z}\right)+k\right)= \pm \ell^{2} I_{0}\left(\left|z_{0}\right| / \ell\right) \quad \text { and } \quad \sigma\left(l_{r}(z-\tilde{z})+k\right) \leqslant \pm \ell^{2} I_{0}(|z| / \ell) \text { for all } z \in \mathbb{D}
$$

where \pm is either always + or always - , and $I_{0}$ is a modified Bessel function of the first kind.

Proof. Throughout the proof, $x \gg 1$ means that $x$ is larger than a large absolute constant. Let

$$
I(z)=\ell^{2}\left(I_{0}(|z| / \ell)-1\right) .
$$

Replacing $k$ by $k-\ell^{2} / \sigma$, the claim $(\mathrm{B} .30)$ is equivalent to the claim

$$
\sigma\left(l_{r}\left(z_{0}-\tilde{z}\right)+k\right)=I\left(z_{0}\right) \quad \text { and } \quad \sigma\left(l_{r}(z-\tilde{z})+k\right) \leqslant I(z) \text { for all } z \in \mathbb{D} \text {. }
$$

For the right-hand side, for $\ell \gg 1$, we have

$$
I(z)=\frac{1}{4}|z|^{2}(1+\mathrm{O}(|z| / \ell)), \quad \nabla I(z)=\left(\frac{1}{2}+O(1 / \ell)\right) z, \quad \nabla^{2} I(z)=\frac{1}{2} \mathbf{1}_{2 \times 2}+\mathrm{O}(1 / \ell) .
$$


For $\ell \gg 1$, the map $z \mapsto \sigma \nabla l_{r}(z)$ takes $B_{r}(0)$ onto $B_{\sigma\left|\nabla Y^{\ell}(r)\right|}(0) \supset B_{\sigma(1-\varepsilon) / r}(0) \supset B_{1}(0)$. Thus, by appropriate choice of $\tilde{z}$ and $k$, the derivatives of $\sigma l_{r}(z-\tilde{z})$ and $\pm I$ can be matched at any $\left|z_{0}\right|<1$. It remains to show the inequality in $(\mathrm{B} .30)$. By definition of $l_{r}$ and since, by $(\overline{\mathrm{B} .3})$, the derivatives of $Y^{\ell}(z)$ are well approximated by those of $-\log |z|$ for $\ell \gg 1$, we have

$$
\nabla^{2} l_{r}(z)=-\frac{1}{r^{2}}\left(\mathbf{1}_{2 \times 2}+\mathrm{O}(1 / \ell)\right) \text { for }|z|<r .
$$

Together with (B.33), using that $1 / r^{2}>1>1 / 2$, it follows that the function $l_{r}(z-\tilde{z})+k$ stays below $\pm I(z)$ for $|z-\tilde{z}|<r$, provided that $\ell \gg 1$. Using further that $l_{r}(0)-l_{r}(r)=\frac{1}{2}+\mathrm{O}(1 / \ell)$, we can choose $\sigma \geqslant \sigma_{0}$ and $\ell \geqslant \ell_{0}$ large enough that

$$
\sigma\left(l_{r}(0)-l_{r}(r)\right)>\frac{1}{4}(1+\mathrm{O}(1 / \ell))=\sup _{\mathbb{D}}( \pm I)-\inf _{\mathbb{D}}( \pm I) .
$$

Since $\sigma\left(l_{r}(0)+k\right) \leqslant \sup _{\mathbb{D}}( \pm I)$, it follows that $\sigma\left(l_{r}(z-\tilde{z})+k\right) \leqslant \inf _{\mathbb{D}}( \pm I)$ for $|z-\tilde{z}|=r$. Since $l_{r}(z-\tilde{z})$ is decreasing in $|z-\tilde{z}|$ the inequality then holds on all of $\mathbb{D}$.

B.2. Perturbed Yukawa equilibrium measure. As in $[6]$, to prove the local law, we will condition on the particles outside small disks. To handle this conditioning, we next state adaptations of the results of [6, Section 3.3] to the Yukawa case. As in [6, Section 3.3], we can assume here that $S_{V}=\rho \overline{\mathbb{D}}$ for some $\rho>0$, where $\mathbb{D} \subset \mathbb{C}$ is the open unit disk. Furthermore, we assume the density of $\mu_{V}$ is bounded below by $\frac{1}{4 \pi} \alpha$ in $\rho \mathbb{D}$ for some parameter $\alpha>0$. The class of perturbed potentials $W$ that we consider is as follows. Let $\nu$ be a positive measure with $\operatorname{supp} \nu \cap \rho \mathbb{D}=\varnothing$, $t>0$ and let $R \in \mathscr{C}(\rho \overline{\mathbb{D}})$ satisfy $\left(\Delta-m^{2}\right) R=0$ in $\rho \mathbb{D}$. Then $W$ is given by

$$
W(z)= \begin{cases}t V(z)+2 U_{\nu}^{\ell}(z),+2 R(z), & z \in \rho \overline{\mathbb{D}} \\ \infty, & z \in \rho \mathbb{D}^{*}\end{cases}
$$

where we write $\mathbb{D}^{*}=\mathbb{C} \backslash \overline{\mathbb{D}}$ for the open complement of the unit disk. Both perturbations $U_{\nu}^{\ell}$ and $R$ are $m$-harmonic inside $\rho \mathbb{D}$, i.e., $\left(\Delta-m^{2}\right) R=0$ and analogously for $U_{\nu}^{\ell}$. In particular, by (2.13), this implies that the density of $\mu_{W}$ is equal to $t \mu_{V}+$ constant in $S_{W}$. For $z \in \partial(\rho \mathbb{D})$ we write $\bar{n}=\bar{n}(z)=z /|z|$ for the outer unit normal, and we write $\partial_{n}^{-} f(z)=\lim _{\varepsilon \downarrow 0} \frac{f(z)-f(z-\varepsilon \bar{n})}{\varepsilon}$ for the derivative in the direction $\bar{n}$ taken from inside $\rho \mathbb{D}$.

The next two propositions show that the bulk of the equilibrium measure $\mu_{V}$ is stable under suitable perturbations $W$ of the form (B.35), and that the density of $\mu_{W}$ on the boundary remains bounded. To prove the stability of the bulk we use the obstacle problem characterization (B.1) of the support.

Proposition B.6. Suppose that $V$ and $W$ are as above (B.35). Then, for any $\ell>0$, the support $S_{W}$ of the equilibrium measure with Yukawa interaction of range $\ell$ and potential $W$ satisfies

$$
S_{W} \supset\left\{z \in \rho \mathbb{D}: \operatorname{dist}\left(z, \rho \mathbb{D}^{*}\right) \geqslant \kappa\right\}, \quad \text { where } \kappa=C \sqrt{\frac{\max \left(\|\nu\|, \rho\left\|\partial_{n}^{-} R\right\|_{\infty, \partial \rho \mathbb{D}}+(t-1)\right)}{\alpha t}} .
$$

Proof. As in the proof of [6. Proposition 3.3], except that we must now replace $\ell$ by $\ell / \rho$, we may assume that $\rho=1$, and we define $D=\left\{z \in \mathbb{D}: \operatorname{dist}\left(z, \mathbb{D}^{*}\right) \geqslant \kappa\right\}$. The replacement of $\ell$ does not matter since the estimate is uniform in $\ell$. By Proposition B.1, to prove the proposition, it suffices to exhibit, for any $z_{0} \in D$, a test function $v_{z_{0}}=v=-U_{\nu}^{\ell}(z)+c$ with $v\left(z_{0}\right)=\frac{1}{2} W\left(z_{0}\right)$ and satisfying the requirements for the potential in (B.1) with $W$ instead of $V$. 
This test function is chosen almost exactly as in the Coulomb case, with the small difference in the handling of the perturbation $R$. Indeed, recall that by assumption $R=U_{\mu}^{\ell}$ for a (signed) charge distribution $\mu$ supported in $\mathbb{D}^{*}$. Up to an additive constant, we may replace $\mu$ by its balayage $\omega$ onto $\partial \mathbb{D}$, i.e., we choose the measure $\omega$ supported on $\partial \mathbb{D}$ such that $R=U_{\omega}^{\ell}+c$ in $\mathbb{D}$. The existence of $\omega$ follows as in the Coulomb case; see e.g. [43]. We choose $\ell_{0}$ to be the sufficiently large absolute constant from Lemma B.5. For $\ell \geqslant \ell_{0}$, we decompose $\omega=\omega_{0}+\omega_{+}-\omega_{-}$ with $\omega_{0}$ a measure of constant density with respect to the arclength measure on $\partial \mathbb{D}$ such that $\int \mathrm{d} \omega=\int \mathrm{d} \omega_{0}$ and with $\omega_{ \pm}$positive measures. For $\ell<\ell_{0}$, we simply decompose $\omega=\omega_{+}-\omega_{-}$ with $\omega_{ \pm}$positive measures and set $\omega_{0}=0$. In both cases, Lemma B.2 implies that the total charge of $\omega_{ \pm}$is estimated by

$$
\left\|\omega_{ \pm}\right\|=\mathrm{O}(1)\left\|\partial_{n}^{-} R\right\|_{\infty, \partial \mathbb{D}}
$$

Then, similarly as in [6, Proposition 3.3], we will choose the function $v$ of the form

$$
v(z)=t u_{V, \ell}(z)+\sigma L(z)+\gamma L_{0}(z)-U^{\omega_{-}}(z), \quad L(z)=\int\left(l_{r}(z-\tilde{z}(w))+k(w)\right)\left(\nu+\omega_{+}\right)(\mathrm{d} w),
$$

where $\sigma>0, r>0, k: \operatorname{supp} \nu \rightarrow \mathbb{R}$ and $\tilde{z}: \operatorname{supp} \nu \rightarrow \mathbb{D}$ are parameters, and the function $l_{r}$ is now defined by (B.18), and $L_{0}(z)$ is chosen of the form

$$
L_{0}(z)=l_{r}\left(z-\tilde{z}_{0}\right)-k_{0}
$$

for some $\tilde{z}_{0} \in \mathbb{C}$ and $k_{0} \in \mathbb{R}$ to be chosen later.

Step 1. With the choice

$$
\gamma=\mathrm{O}(1)\left\|\partial_{n}^{-} R\right\|_{\infty, \partial \mathbb{D}}, \quad \sigma=\max \left(\frac{1}{2}, \frac{(t-1)-\gamma+\left\|\omega_{-}\right\|}{\left\|\nu+\omega_{+}\right\|}\right), \quad r=2 \sqrt{\frac{\left\|\nu+\omega_{+}\right\| \sigma+\gamma}{\alpha t}}=\frac{1}{2} \kappa,
$$

the function $v$ is of the form $-U_{\mu}^{\ell}+c$ for a positive measure $\mu$ of total mass at most $t+\left\|\omega_{-}\right\|-\gamma-$ $\sigma\left\|\nu+\omega_{+}\right\| \leqslant 1$. Indeed, by definition, $-t u_{V, \ell}+U_{\omega_{-}}$is the potential of a positive measure of mass $t+\left\|\omega_{-}\right\|$and $-\sigma L-\gamma L_{0}$ is the potential of a negative measure of total mass $-\sigma\left\|\nu+\omega_{+}\right\|-\gamma$. Their sum is the potential of a positive measure since

$$
\left(\Delta-m^{2}\right)\left(t u_{V, \ell}-U^{\omega_{-}}+\sigma L+\gamma L_{0}\right) \geqslant 2 \pi t \rho_{V, \ell}+2 \pi \omega_{-}-\frac{2 \sigma}{r^{2}}\left\|\nu+\omega_{+}\right\|-\frac{2 \gamma}{r^{2}} \geqslant 0,
$$

where we used the assumption $\rho_{V, \ell} \geqslant \alpha /(4 \pi)$.

Step 2. For appropriate choice of the parameters $\tilde{z}$ and $k$ (depending on $z_{0}$ ), we have $v\left(z_{0}\right)=$ $\frac{1}{2} W\left(z_{0}\right)$ and $v \leqslant \frac{1}{2} W$ in $\overline{\mathbb{D}}$. Indeed, replacing [6, Lemma 3.6] by Lemma B.4 stated below the proof, we choose the parameters $\tilde{z}$ and $k$ exactly as in the proof of [6, Proposition 3.3] to achieve

$$
\begin{aligned}
\sigma L(z) & \leqslant \frac{1}{2} \int Y^{\ell}(z-w)\left(\nu+\omega_{+}\right)(d w) \quad \text { for all } z \in \overline{\mathbb{D}} \\
\sigma L\left(z_{0}\right) & =\frac{1}{2} \int Y^{\ell}\left(z_{0}-w\right)\left(\nu+\omega_{+}\right)(d w) .
\end{aligned}
$$

This concludes the proof for $\ell<\ell_{0}$. For $\ell \geqslant \ell_{0}$, it remains to handle the remaining part of the perturbation, which is the potential $U_{\bar{\omega}}^{\ell}$ generated by the constant part $\bar{\omega}$ of $\omega$. Since the Yukawa potential of $\bar{\omega}$ is $m$-harmonic in $|z|<1$, radially symmetric and bounded as $|z| \rightarrow 0$, as in $\mathrm{B.22}$, it is explicitly given inside $\mathbb{D}$ by

$$
U_{\bar{\omega}}^{\ell}(z)= \pm A \ell^{2} I_{0}(|z| / \ell) \quad(|z|<1),
$$


for some constant $A>0$ depending on $\ell$ and $\bar{\omega}$, where $I_{n}$ are the modified Bessel functions of the first kind. Using that $I_{0}^{\prime}=I_{1}$ by general relations between Bessel functions,

$$
\nabla U_{\bar{\omega}}^{\ell}(z)= \pm A 2 \ell I_{1}(|z| / \ell) \frac{z}{|z|}=\partial_{n}^{-} U_{\bar{\omega}}^{\ell}(1) \frac{I_{1}(|z| / \ell)}{I_{1}(1 / \ell)} \frac{z}{|z|} .
$$

The modified Bessel functions satisfy the asymptotics

$$
I_{0}(t) \sim 1+\frac{1}{4} t^{2}, \quad I_{1}(t) \sim \frac{1}{2} t, \quad \text { as } t \rightarrow 0 .
$$

Therefore, with (B.8), the constant $A$ is given by

$$
A= \pm \frac{\partial_{n}^{-} U_{\bar{\omega}}^{\ell}(1)}{2 \ell I_{1}(1 / \ell)}= \pm(1+\mathrm{O}(1 / \ell)) \partial_{n}^{-} U_{\bar{\omega}}^{\ell}(1) \leqslant(1+\mathrm{O}(1 / \ell))\left\|\partial_{n}^{-} U_{\omega}^{\ell}\right\|_{\infty, \partial \mathbb{D}}=\mathrm{O}(1)\left\|\partial_{n}^{-} R\right\|_{\infty, \partial \mathbb{D}}
$$

By Lemma B.5, there exists a large constant $\sigma$ such that we can choose $k_{0}$ and $\tilde{z}_{0}$ and $\gamma=$ $\mathrm{O}(1)\left\|\partial_{n}^{-} R\right\|_{\infty, \partial \mathbb{D}}$ such that with $\gamma=\sigma A$,

$$
\gamma L_{0}\left(z_{0}\right)=U_{\bar{\omega}}^{\ell}\left(z_{0}\right), \quad \gamma L_{0}(z) \leqslant U_{\bar{\omega}}^{\ell}(z) \quad \text { for all } z \in \mathbb{D} .
$$

This concludes the proof.

Proposition B.7. Suppose that $V$ and $W$ are as above (B.35) and assume in addition that $\mu_{V}$ is absolutely continuous with respect to the 2-dimensional Lebesgue measure. Then $\mu_{W}=\mu+\eta$, where $\mu$ is absolutely continuous with respect to $\mu_{V}$, and $\eta$ absolutely continuous with respect to the arclength measure $s$ on $\partial \rho \mathbb{D}$ with the Radon-Nikodym derivative bounded by

$$
\rho\left\|\frac{d \eta}{d s}\right\|_{\infty} \leqslant C\left(\|\eta\|+\|\nu\|+2 \rho\left\|\partial_{n}^{-} R\right\|_{\infty, \partial \rho \mathbb{D}}+|1-t| \rho\left\|\partial_{n}^{-} V\right\|_{\infty, \partial \rho \mathbb{D}}\right) .
$$

Proof. The only change in the proof of Proposition B.7 compared to [6] is the change of the logarithmic potentials to Yukawa potentials. In particular, the formula (B.4) holds and $\nabla Y^{\ell}(z)$ is proportional to $\nabla \log \frac{1}{|z|}$.

B.3. One-step estimate for the Yukawa interaction. As in [6. Proposition 4.1], we use a simple mean-field partition function estimate to obtain a bound on the fluctuations of smooth linear statistics. In the following, $\mathrm{d} m$ denotes the Lebesgue measure and is not related to the mass $m$.

Proposition B.8. Let $\Sigma=\Sigma_{W}$ be a smooth domain with boundary $\partial \Sigma$ or $\Sigma=\mathbb{T}$ (with $\partial \Sigma=\emptyset$ ). Given a potential $W \in C_{l o c}^{1,1}\left(\Sigma_{W}\right)$ possibly depending on the number of particles $M$, assume that there exist $u: \Sigma_{W} \rightarrow \mathbb{R}_{+}$and $v: \partial \Sigma_{W} \rightarrow \mathbb{R}_{+} \quad\left(\right.$ if $\partial \Sigma_{W} \neq \emptyset$ ) such that $d \mu_{W}=u \mathrm{~d} m+v \mathrm{~d} s$, where $\mathrm{d} m$ is the 2-dimensional Lebesgue measure and $\mathrm{d} s$ is the arclength measure on $\partial \Sigma_{W}$ (if $\left.\partial \Sigma_{W} \neq \emptyset\right)$. Assume the conditions (i)-(iv) as stated in [6. Proposition 4.1] but replace the bounds on $\frac{1}{4 \pi} \Delta W$ (which is the density in the Coulomb case) more generally by the same bound on the density of the equilibrium measure $u$ and also modify the assumption (iv) by replacing $\zeta$ by $\zeta^{\ell}=U_{\mu_{W}}^{\ell}+\frac{1}{2} V-c_{V}$, where the constant $c_{V}$ is the one in (2.13). Then, for any constant $A$, for any bounded $f \in C^{2}(\mathbb{C})$ with compactly supported $\left(\Delta-m^{2}\right) f$,

$$
\begin{aligned}
\log \int \mathrm{e}^{-\beta H_{M, W}(\mathbf{z})+\sum f\left(z_{j}\right)} m(\mathrm{~d} \mathbf{z}) \leqslant & -\beta M^{2} I_{W}^{\ell}\left(\mu_{V}\right)+M\left(f, \mu_{W}\right)+\frac{1}{8 \pi \beta}\left(f,-\left(\Delta-m^{2}\right) f\right) \\
& +\mathrm{O}\left(M^{-A}\right)\|\Delta f\|_{\infty}+\mathrm{O}(M \log M), \\
\log \int \mathrm{e}^{-\beta H_{M, W}(\mathbf{z})} m(\mathrm{~d} \mathbf{z}) \geqslant- & \beta M^{2} I_{W}^{\ell}\left(\mu_{V}\right)+\mathrm{O}(M \log M),
\end{aligned}
$$


and consequently for any $\xi \geqslant 1+1 / \beta$,

$$
\left|\sum_{j} f\left(z_{j}\right)-M \int f \mathrm{~d} \mu_{W}^{\ell}\right|=\mathrm{O}(\xi)\left(\sqrt{M \log M}\left(f,\left(-\Delta+m^{2}\right) f\right)^{1 / 2}+M^{-A}\|\Delta f\|_{\infty}\right),
$$

with probability at least $1-\mathrm{e}^{-\xi \beta M \log M}$, with the implicit constant depending only on the numbers $A$ in the assumptions (i)-(iv).

Proof. The probability estimate is obtained as in [6] from the partition function bounds $($ B.46) and (B.47), which are analogous to 6 , Lemmas 4.3 and 4.4] except that $\|\nabla f\|_{2}=(f,(-\Delta) f)^{1 / 2}$ is replaced by $\left(f,\left(-\Delta+m^{2}\right) f\right)^{1 / 2}$. The lower bound can be proved exactly the same way; for the upper bound we may bound the energy slightly differently from below, as follows, avoiding the need that the support of $\left(\Delta-m^{2}\right) f$ is contained in $S_{V}$.

All the properties of the Coulomb potential used in the proof of [6, Lemmas 4.3] also hold for the Yukawa potential and on the torus. Replacing the point charges by charged disks of radius $\varepsilon$, and denoting by $D^{\ell}(\cdot, \cdot)$ the Yukawa analog of $D(\cdot, \cdot)$, we get the bound

$$
\begin{aligned}
H_{M}^{\ell}(\mathbf{z})- & \frac{1}{\beta M} \sum_{j} f\left(z_{j}\right) \geqslant M^{2} D^{\ell}\left(\hat{\mu}^{(\varepsilon)}, \hat{\mu}^{(\varepsilon)}\right)+M^{2}\left(W-\frac{1}{\beta M} f, \hat{\mu}\right)+\mathrm{O}\left(M \log \frac{1}{\varepsilon}\right) \\
& =M^{2}\left(D^{\ell}\left(\hat{\mu}^{(\varepsilon)}, \hat{\mu}^{(\varepsilon)}\right)+(W, \hat{\mu})-\left(\frac{1}{\beta M} f, \hat{\mu}^{(\varepsilon)}\right)\right)+M^{2}\left(\frac{1}{\beta M} f, \hat{\mu}^{(\varepsilon)}-\hat{\mu}\right)+\mathrm{O}\left(M \log \frac{1}{\varepsilon}\right) .
\end{aligned}
$$

Writing

$$
D^{\ell}\left(\hat{\mu}^{(\varepsilon)}, \hat{\mu}^{(\varepsilon)}\right)=D^{\ell}\left(\mu_{W}, \mu_{W}\right)+2 D^{\ell}\left(\mu_{W}, \hat{\mu}^{(\varepsilon)}-\mu_{W}\right)+D^{\ell}\left(\hat{\mu}^{(\varepsilon)}-\mu_{W}, \hat{\mu}^{(\varepsilon)}-\mu_{W}\right)
$$

and further using the Euler-Lagrange equation (2.13) to write

$$
2 D^{\ell}\left(\mu_{W}, \hat{\mu}^{(\varepsilon)}-\mu_{W}\right)+(W, \hat{\mu})=\left(W, \mu_{W}\right)+2\left(\zeta^{\ell}, \hat{\mu}-\mu_{W}\right)+2\left(U_{\mu_{W}}^{\ell}, \hat{\mu}^{(\varepsilon)}-\hat{\mu}\right),
$$

where $\zeta^{\ell}=U_{\mu_{W}}^{\ell}+\frac{1}{2} W-c_{W}=0$ on $S_{W}$, we therefore can the bound $H_{M}^{\ell}(\mathbf{z})-\frac{1}{\beta M} \sum_{j} f\left(z_{j}\right)$ by

$$
\begin{aligned}
M^{2}\left(I_{W}^{\ell}\left(\mu_{W}\right)+D^{\ell}\left(\hat{\mu}^{(\varepsilon)}-\mu_{W},\right.\right. & \left.\left.\hat{\mu}^{(\varepsilon)}-\mu_{W}\right)-\left(\frac{1}{\beta M} f, \hat{\mu}^{(\varepsilon)}\right)\right)+2 M^{2}\left(\zeta^{\ell}, \hat{\mu}-\mu_{W}\right) \\
& +M^{2}\left(\frac{1}{\beta M} f, \hat{\mu}^{(\varepsilon)}-\hat{\mu}\right)+2 M^{2}\left(U_{\mu_{W}}^{\ell}, \hat{\mu}^{(\varepsilon)}-\hat{\mu}\right)+\mathrm{O}\left(M \log \varepsilon^{-1}\right) .
\end{aligned}
$$

We write

$$
D^{\ell}\left(\hat{\mu}^{(\varepsilon)}-\mu_{W}, \hat{\mu}^{(\varepsilon)}-\mu_{W}\right)-\left(\frac{1}{\beta M} f, \hat{\mu}^{(\varepsilon)}\right)=\frac{1}{2 \pi}\left(\frac{1}{\beta M} f+U_{\hat{\mu}^{(\varepsilon)}-\mu_{W}}^{\ell},-\left(\Delta-m^{2}\right) U_{\hat{\mu}^{(\varepsilon)}-\mu_{W}}^{\ell}\right)-\left(\frac{1}{\beta M} f, \mu_{W}\right) .
$$

The Yukawa potentials decay exponentially at infinity, so we may integrate by parts and use the elementary inequality $-|a b|+|b|^{2} \geqslant-|a|^{2} / 4$ to get

$$
\begin{aligned}
\frac{1}{2 \pi}\left(\frac{1}{\beta M} f+U_{\hat{\mu}^{(\varepsilon)}-\mu_{W}}^{\ell},(-\Delta) U_{\hat{\mu}^{(\varepsilon)}-\mu_{W}}^{\ell}\right) & =\frac{1}{2 \pi}\left(\frac{1}{\beta M} \nabla f+\nabla U_{\hat{\mu}^{(\varepsilon)}-\mu_{W}}^{\ell}, \nabla U_{\hat{\mu}^{(\varepsilon)}-\mu_{W}}^{\ell}\right) \\
& \geqslant-\frac{1}{8 \pi \beta^{2} M^{2}}(\nabla f, \nabla f)=-\frac{1}{8 \pi \beta^{2} M^{2}}(f,(-\Delta) f) .
\end{aligned}
$$

By the same inequality we have

$$
\frac{1}{2 \pi}\left(\frac{1}{\beta M} f+U_{\hat{\mu}^{(\varepsilon)}-\mu_{W}}^{\ell}, m^{2} U_{\hat{\mu}^{(\varepsilon)}-\mu_{W}}^{\ell}\right) \geqslant-\frac{m^{2}}{8 \pi \beta^{2} M^{2}}(f, f) .
$$


In conclusion,

$$
\begin{aligned}
& M^{2} D^{\ell}\left(\hat{\mu}^{(\varepsilon)}, \hat{\mu}^{(\varepsilon)}\right)+M^{2}\left(W-\frac{1}{\beta M} f, \hat{\mu}\right) \\
& \geqslant M^{2}\left(I_{W}^{\ell}\left(\mu_{W}\right)-\frac{1}{\beta M}\left(f, \mu_{W}\right)-\frac{1}{8 \pi \beta^{2} M^{2}}\left(f,-\left(\Delta-m^{2}\right) f\right)\right) \\
& \quad+2 M^{2}\left(\zeta^{\ell}, \hat{\mu}-\mu_{W}\right)+M^{2}\left(\frac{1}{\beta M} f, \hat{\mu}^{(\varepsilon)}-\hat{\mu}\right)+2 M^{2}\left(U_{\mu_{W}}^{\ell}, \hat{\mu}^{(\varepsilon)}-\hat{\mu}\right)+\mathrm{O}\left(M \log \frac{1}{\varepsilon}\right) .
\end{aligned}
$$

In the same way as in [6, for the error terms on the last line,

$$
\frac{M}{\beta}\left|\left(f^{(\varepsilon)}-f, \hat{\mu}\right)\right| \leqslant \frac{M}{\beta} C \varepsilon^{2}\|\Delta f\|_{\infty} \leqslant M^{-A}\|\Delta f\|_{\infty}
$$

and

$$
2 M^{2}\left|\left(U_{\mu_{W}^{\ell}}, \hat{\mu}^{(\varepsilon)}-\hat{\mu}\right)\right| \leqslant C \varepsilon^{2} M^{A_{u}}+C \sqrt{\varepsilon} M^{A_{v}} \leqslant 1,
$$

by choosing $\varepsilon$ sufficiently small depending on $A$ and such that $\log \frac{1}{\varepsilon}=\mathrm{O}(\log M)$. Finally, we use that $2 M^{2}\left(\zeta^{\ell}, \hat{\mu}-\mu_{W}\right) \geqslant 0$ by the Euler-Lagrange equation to conclude the proof.

Remark B.9. For test functions $f$ supported in $S_{V}^{\ell}$ and satisfying the condition $\int f \mathrm{~d} m=0$,

$$
\int f \mathrm{~d} \mu_{V}^{\ell}=\int f \frac{1}{4 \pi}\left(\Delta V-m^{2} V\right) \mathrm{d} m .
$$

(We recall that $\mathrm{d} m$ is the Lebesgue measure and not related to the mass $m$.) Consequently, if $V$ is replaced by $V+R$ with $\left(\Delta-m^{2}\right) R=0$, and assuming that $f$ is supported in the intersection of the supports of the equilibrium measures of $V$ and $V+R$, and that $\int f \mathrm{~d} m=0$, we have

$$
\int f \mathrm{~d} \mu_{V}^{\ell}=\int f \mathrm{~d} \mu_{V+R}^{\ell}
$$

Since we are ultimately interested in test functions without the condition $\int f \mathrm{~d} m=0$, some additional care is required. (The condition was not necessary in the Coulomb case in [6].) This problem will be addressed at the beginning of the proof of Proposition B.10.

B.4. Yukawa gas on the torus: proof of Theorem 2.2, We follow the proof of 6 , Theorem 1.1] to improve the estimate of Proposition B.8 to the stronger one asserted by Theorem 2.2 by using local conditioning. Compared with [6, Theorem 1.1], there are two main changes in Theorem 2.2. (i) the domain is now a torus rather than the plane, (ii) the interaction is the Yukawa potential rather than the Coulomb potential. The domain change is only visible in the first step of the induction in the proof; it does not have any effect after the first step when we take local conditioning. The change from the Coulomb potential to the Yukawa potential does require changes in the local conditioning; it will be taken into account by replacing the potential theory estimates in 6 by their generalizations in Sections B.1 B.2.

First, we note that [6. Section 5] applies without changes except that the Coulomb potential $\log 1 /|z|$ is replaced by the Yukawa potential $Y^{\ell}(z)$ in all expressions, and with the additional condition that $\int f \mathrm{~d} m=0$ in the assumption of [6, Proposition 5.3]. This condition is necessary because, with the $m$-harmonic perturbation $V_{o}$, inside the support of $\mu_{W}$ we now have

$$
\mu_{W}=\frac{N}{M} \mu_{V}+\text { const. }
$$

by (2.14). As explained in Remark B.9, the additional constant has no effect if both sides are integrated against a test function $f$ with support in the support of $\mu_{W}$ that satisfies $\int f \mathrm{~d} m=0$. 
Next, we adapt [6, Section 6] to the Yukawa case. Here two modifications are required. First, the scaling of the Yukawa gas is different, which leads to a different recursion of scales. Second, in the case of the Yukawa gas, as noted above, the density of the equilibrium is only stable under $m$-harmonic perturbations up to a constant, and thus a small extra argument is required to remove the mean zero condition.

As previously, we write $\ell=N^{-1 / 2+\delta}$ for the range of the Yukawa potential. Given $\varepsilon>0$ (and assuming $\varepsilon<\delta$ ), we set $s_{0}=0$ and

$$
s_{j+1}=\left(\left(\frac{1}{4}+\frac{s_{j}}{2}\right) \wedge\left(s_{j}+\delta\right)\right)-\varepsilon,
$$

for $\varepsilon>0$ fixed sufficiently small. As long as the second term in the minimum above dominates, the sequence $s_{j}$ grows linearly as $j(\delta-\varepsilon)$ until the scale $s=\frac{1}{2}-2 \delta$ is reached. After that, the first term dominates. Then $s_{j}$ evolves according to $\frac{1}{2}-\delta-\varepsilon$; then $\frac{1}{2}-\frac{1}{2} \delta$; then $\frac{1}{2}-\frac{1}{4} \delta-\frac{3}{2} \varepsilon$ and converges geometrically to $\frac{1}{2}-2 \varepsilon$. In particular, given $s \in\left(0, \frac{1}{2}\right)$, we can fix $\varepsilon>0$ and $n<\infty$ such that $s_{n}=s$, and we will assume such a choice from now on.

The induction assumption $\left(\mathrm{A}_{r}\right)$ is modified as follows (as a formal remark, note that compared to [6], we changed the index of the condition $A_{t}$ into $A_{r}$ as, in the current paper, $t$ refers to the argument of the Laplace transform).

Assumption $\left(\mathbf{A}_{r}\right)$. For any bounded $f \in C^{2}(\mathbb{T})$ with $\operatorname{supp}\left(\Delta-m^{2}\right) f \subset B_{r}^{\circ} \cap S_{V}$, we have

$$
\left|\frac{1}{N} \sum_{j} f\left(z_{j}\right)-\int f \mathrm{~d} \mu_{V}\right| \prec N^{-\frac{1}{2}-r}\left(f,\left(-\Delta+m^{2}\right) f\right)^{\frac{1}{2}}+N^{-1-2 r}\|\Delta f\|_{\infty} .
$$

Proposition B.10. For arbitrary $\varepsilon>0,\left(A_{r}\right)$ implies $\left(A_{s}\right)$ for any $0 \leqslant r \leqslant s \leqslant\left(\frac{1}{4}+\frac{1}{2} r\right) \wedge(r+\delta)-\varepsilon$ (with the implicit constants depending on $\varepsilon$ ).

Proof. First, we show that, for any $s$ as asserted in the proposition, it suffices to prove that $\left(\mathrm{A}_{r}\right)$ implies $\left(\mathrm{A}_{s}^{\prime}\right)$, where $\left(\mathrm{A}_{s}^{\prime}\right)$ is defined exactly as $\left(\mathrm{A}_{s}\right)$ except that the test functions $f$ are required to obey the additional mean zero condition $\int f \mathrm{~d} m=0$. Indeed, assume $\left(\mathrm{A}_{r}\right)$ and that we have proved $\left(\mathrm{A}_{s}^{\prime}\right)$ for all $s$ as in the statement of the proposition. Recall from above that $B=B_{s}$ is a disk of radius $N^{-s}$ and that $B_{s}^{\circ}$ the disk with the same center and half the radius. For any test function $f$ supported on $B_{s}^{\circ}$ we define $f_{i}(z)=2^{-2 i} f\left(2^{-i} z\right)$, and write

$$
f=f_{k}+\sum_{i=0}^{k-1}\left(f_{i}-f_{i+1}\right),
$$

where $k$ is the largest integer such that $2^{k} N^{-s} \leqslant N^{-t}$. Then

$$
\left\|\Delta f_{i}\right\|_{\infty}=2^{-4 i}\|\Delta f\|_{\infty}, \quad\left(f_{i},\left(-\Delta+m^{2}\right) f_{i}\right) \leqslant 2^{-2 i}\left(f,\left(-\Delta+m^{2}\right) f\right) .
$$

Therefore, with $s_{i}=s-(i+1) / \log _{2} N$ for $i=0,1, \ldots, k-1$, applying $\left(\mathrm{A}_{s_{i}}^{\prime}\right)$ to the mean zero function $f_{i}-f_{i+1}$, we obtain

$$
\begin{aligned}
& \frac{1}{N} \sum_{j}\left(f_{i}\left(z_{j}\right)-f_{i+1}\left(z_{j}\right)\right)-\int\left(f_{i}-f_{i+1}\right) \mathrm{d} \mu_{V} \\
& \prec 2^{2 i} N^{-1-2 s}\left\|\Delta\left(f_{i}-f_{i+1}\right)\right\|_{\infty}+2^{i} N^{-\frac{1}{2}-s}\left(f_{i}-f_{i+1},\left(-\Delta+m^{2}\right)\left(f_{i}-f_{i+1}\right)\right)^{1 / 2} \\
& \prec 2^{-2 i} N^{-1-2 s}\|\Delta f\|_{\infty}+N^{-\frac{1}{2}-s}\left(f,\left(-\Delta+m^{2}\right) f\right)^{1 / 2} .
\end{aligned}
$$


Similarly, applying $\left(\mathrm{A}_{r}\right)$ to $f_{k}$, we have

$$
\begin{aligned}
\frac{1}{N} \sum_{j} f_{k}\left(z_{j}\right)-\int f_{k} \mathrm{~d} \mu_{V} & \prec\left(N^{-1-2 r}\left\|\Delta f_{k}\right\|_{\infty}+N^{-\frac{1}{2}-r}\left(f_{k},\left(-\Delta+m^{2}\right) f_{k}\right)^{1 / 2}\right) \\
& \prec\left(2^{-4 k} N^{-1-2 r}\|\Delta f\|_{\infty}+2^{-k} N^{-\frac{1}{2}-r}\left(f,\left(-\Delta+m^{2}\right) f\right)^{1 / 2}\right) \\
& \prec\left(2^{-4 k} N^{-1-2 s}\|\Delta f\|_{\infty}+2^{-k} N^{-\frac{1}{2}-s}\left(f,\left(-\Delta+m^{2}\right) f\right)^{1 / 2}\right) .
\end{aligned}
$$

Then

$$
\begin{aligned}
\frac{1}{N} \sum_{j} f\left(z_{j}\right)-\int f & =\frac{1}{N} \sum_{j}\left(f_{k}\left(z_{j}\right)+\sum_{i=0}^{k-1}\left(f_{i}\left(z_{j}\right)-f_{i+1}\left(z_{j}\right)\right)\right)-\int\left(f_{k}+\sum_{i=0}^{k-1}\left(f_{i}-f_{i+1}\right)\right) \\
& \prec \sum_{i=0}^{k}\left(2^{-2 i} N^{-1-2 s}\|\Delta f\|_{\infty}+N^{-\frac{1}{2}-s}\left(f,\left(-\Delta+m^{2}\right) f\right)^{1 / 2}\right) \\
& \prec N^{-1-2 s}\|\Delta f\|_{\infty}+N^{-\frac{1}{2}-s}\left(f,\left(-\Delta+m^{2}\right) f\right)^{1 / 2} .
\end{aligned}
$$

It remains to prove that $\left(\mathrm{A}_{r}\right)$ implies $\left(\mathrm{A}_{s}^{\prime}\right)$ for $s$ as in the statement of the proposition. This proof proceeds exactly as in [6, Section 6.1$]$, with the only essential changes in $[6$, Lemmas $6.2-$ 6.3], since now $m^{2}>0$ in $(B .49$. Indeed, the required properties of the conditional equilibrium measure follow from Propositions B.6 B.7, as soon as [6, Lemmas 6.2-6.3] are adapted.

In 6, Lemma 6.2], which states that $\tau=1+\mathrm{O}\left(N^{-c \varepsilon}\right)$ (where we recall that $\tau=\frac{N}{M} \mu_{V}(B)$ ) and $\nu(\widetilde{\mathbb{C}})=\mathrm{O}\left(N^{-c \varepsilon}\right)$, with high probability, the following changes are necessary. Recall that $\chi_{ \pm}$are smooth cutoff functions with

$$
\left.\chi_{+}\right|_{B}=1,\left.\quad \chi_{+}\right|_{B_{+}^{c}}=0,\left.\quad \chi_{-}\right|_{B^{c}}=0,\left.\quad \chi_{-}\right|_{B_{-}}=1,
$$

obeying $\left\|\nabla^{k} \chi_{ \pm}\right\|_{\infty}=\mathrm{O}\left(N^{k s} / \eta^{k}\right)$ for $k=0,1,2$ (see 6 for the definitions of the expressions). We replace the estimates on $\left(\chi_{ \pm},-\Delta \chi_{ \pm}\right)$by

$$
\left(\chi_{ \pm},\left(-\Delta+m^{2}\right) \chi_{ \pm}\right)=\mathrm{O}\left(\eta N^{-2 s} N^{2 s} / \eta^{2}\right)+\mathrm{O}\left(N^{1-2 \delta} N^{-2 s}\right)=\mathrm{O}(1 / \eta)+\mathrm{O}\left(N^{1-2 \delta-2 s}\right),
$$

and thus

$$
N^{-1-2 r}\left(\chi_{ \pm},\left(-\Delta+m^{2}\right) \chi_{ \pm}\right)=\mathrm{O}\left(N^{-4 s-4 \varepsilon} / \eta\right)+\mathrm{O}\left(N^{-4 s-2 \varepsilon}\right)=\mathrm{O}\left(N^{-4 s-c \varepsilon}\right)
$$

Using this, the rest of the proof of [6, Lemma 6.2] proceeds as in [6].

In [6, Lemma 6.3], which states the estimate $N^{-s}\|\nabla \hat{R}\|_{L^{\infty}(B)}=\mathrm{O}\left(N^{-c \varepsilon}\right)$, with high probability, we make the following changes. We change the definition of $f$ from $f(w)=N^{-s} \nabla\left(\psi(w) \log \frac{1}{|z-w|}\right)$ to $f(w)=N^{-s} \nabla\left(\psi(w) Y^{\ell}(z-w)\right)$. In particular, the property that $\Delta f=0$ on $A^{c}$ is replaced by $\left(\Delta-m^{2}\right) f=0$ on $A^{c}$, and using this, the estimate on $(f,-\Delta f)$ is replaced by (here again we use a notation from $[6]$, namely $\left.a=N^{-c \varepsilon}\right)$,

$$
\begin{aligned}
& N^{-1-2 r}\left(f,\left(-\Delta+m^{2}\right) f\right) \\
& =N^{-1-2 r} \mathrm{O}\left(N^{-2 s} N^{2 s}|\log a| / a^{2}\right)+N^{-1-2 r} \mathrm{O}\left(N^{1-2 \delta} N^{2 s}|\log a|^{2}\right)=\mathrm{O}\left(N^{-4 s-c \varepsilon}\right),
\end{aligned}
$$

so that, again, the rest of the proof of [6, Lemma 6.3] proceeds as in [6]. 
Proof of Theorem 2.2. Proposition B.8 applied to the torus $\Sigma=\mathbb{T}$ and with $M=N$ verifies Assumption $\left(\mathrm{A}_{0}\right)$. We then apply local conditioning, exactly as in the proof of [6, Theorem 1.1]. For the conditioned measure, since $\ell \leqslant N^{-c}$, we may replace the torus Yukawa potential by the full plane Yukawa potential since

$$
H_{N, 0}(z)=\sum_{j \neq k} U^{\ell}\left(z_{j}-z_{k}\right)+\mathrm{O}\left(N^{-\infty}\right)=\sum_{j \neq k} Y^{\ell}\left(z_{j}-z_{k}\right)+\mathrm{O}\left(N^{-\infty}\right)
$$

with error bound uniform in $z \in \mathbb{T}^{N}$. By inductive application of Proposition B.10, the assumption $\left(\mathrm{A}_{s}\right)$ is verified for all $s \in\left(0, \frac{1}{2}\right)$. This completes the proof.

B.5. Coulomb gas on the plane: proof of Theorem 2.3. Theorem 2.3 is generalization of 6 , Theorem 1.1] in the following three ways: (i) the distance of the support of the test function to the boundary of the support of the equilibrium measure can be $\gg N^{-1 / 4}+t^{1 / 4}$ rather than order 1; (ii) the Coulomb potential can be replaced by the perturbed Coulomb potential; (iii) or replaced by the Yukawa potential $Y^{\ell}$ with $\ell \geqslant N^{2}$. We will show that all these changes have only minor effects on the proof. The condition $\gg N^{-1 / 4}+t^{1 / 4}$ arises because $N^{-1 / 4}+t^{1 / 4}$ is the scale that can be controlled without induction. Indeed, the requirement of distance $\gg N^{-1 / 4}$ was already implicit in [6], but the distance requirement was simply estimated crudely by order 1 there. When the perturbation is present, i.e., $t \neq 0$, there is an additional error term which leads to the condition $\gg t^{1 / 4}$; see below.

We begin with the condition on the distance to the boundary. In the proof of [6, Theorem 1.1], in [6. Section 6], by replacing $V(z)$ by $V\left(z-z_{0}\right)$ for some fixed $z_{0} \in S_{V}$, it was sufficient to restrict the induction to functions supported in the centered balls $B_{s}^{o}=B\left(0, \frac{1}{2} N^{-s}\right) \subset B_{s}=B\left(0, N^{-s}\right)$. For test functions whose support has distance $\gg N^{-1 / 4}+t^{1 / 4}$ to the boundary of the support of the equilibrium measure, we now choose $z_{0}$ to be $N$-dependent points with $\operatorname{dist}\left(z_{0}, S_{V}^{c}\right) \gg$ $N^{-1 / 4}+t^{1 / 4}$. This requires no changes in the proof because the initial estimate (here given by Proposition B.12 below) has no restriction on the support of the test function $f$. Writing $t=N^{-2 a}$, in the first inductive step, we can choose the scale as $N^{-s_{1}}$ with $s_{1}=(1 / 4 \wedge a / 2)-\varepsilon$. By assumption the ball of this radius centered at $z_{0}$ is contained in the support of the equilibrium measure and has density bounded below there. Hence there is no change in the remaining steps. Thus the condition $\gg N^{-1 / 4}+t^{1 / 4}$ arises because $N^{-1 / 4}+t^{1 / 4}$ is the scale that the density in that scale can be controlled without induction.

As a preliminary step towards Theorem 2.3 , we prove the following estimate, which provides a weaker fluctuation bound than asserted in Theorem 2.3. However, once this bound is established for all scales, Theorem 2.3 then follows from the same estimates.

Proposition B.11. Assume the same conditions as in Theorem 2.3. Write $t=N^{-2 a}$ and suppose that supp $f$ has diameter at most $N^{-s}$. Then

$$
\frac{X_{f}}{N} \prec\left(N^{-\frac{1}{2}-s}+N^{-a-2 s}\right)\|\nabla f\|_{2}+\left(N^{-1-2 s}+N^{-2 a-4 s}\right)\|\Delta f\|_{\infty} .
$$

To prove this bound, we proceed as in the proof of [6, Theorem 1.1]. The first ingredient is the following generalization of the one-step estimate [6, Proposition 4.1].

Proposition B.12. Assume that the potential $W$ and the number of particles $M$ satisfy the assumptions of [6, Proposition 4.1]. Consider the probability measure on $\Sigma_{W}^{M}$ with density proportional to $\mathrm{e}^{-\beta H(\mathbf{z})}$ where we assume that for some constant $K$ the Hamiltonian $H: \Sigma_{W}^{M} \rightarrow \mathbb{R}$ satisfies the uniform estimate

$$
\left|H(\mathbf{z})-H_{M, W}^{\mathcal{C}}(\mathbf{z})\right| \leqslant t M K
$$


Then for any bounded $f \in C^{2}(\mathbb{C})$ with supp $\Delta f$ compact,

$$
\sum_{j} f\left(z_{j}\right)-M \int f \mathrm{~d} \mu_{W}=\mathrm{O}(\xi)\left((t M K+M \log M)^{1 / 2}\|\nabla f\|_{2}+M^{-A}\|\Delta f\|_{\infty}\right)
$$

with probability at least $1-\mathrm{e}^{-\xi \beta(t M K+M \log M)}$ for any $\xi \geqslant 1+1 / \beta$. The same estimate holds for the Yukawa gas with $\ell \geqslant M^{2}$.

Proof of Proposition B.12. The proof of the proposition is completely parallel to the one without the perturbation $\tilde{G}$, only with an additional error term from (B.51). Namely, by the assumption (B.51), we may trivially estimate

$$
\log \int \mathrm{e}^{-\beta H_{M, W}^{\mathcal{C}}} m^{\otimes M}(\mathrm{~d} \mathbf{z})-t M K \leqslant \log \int \mathrm{e}^{-\beta H} m^{\otimes M}(\mathrm{~d} \mathbf{z}) \leqslant \log \int \mathrm{e}^{-\beta H_{M, W}^{\mathcal{C}} m^{\otimes M}}(\mathrm{~d} \mathbf{z})+t M K .
$$

By [6, Lemmas 4.3-4.4], the partition function of the Coulomb Hamiltonian (without perturbation term) can be estimated as

$$
\begin{aligned}
& \frac{1}{\beta} \log \int \mathrm{e}^{-\beta H_{W}^{\mathcal{C}}} m^{\otimes M}(\mathrm{~d} \mathbf{z}) \geqslant M^{2} I_{W}+\mathrm{O}(M \log M), \\
& \frac{1}{\beta} \log \int \mathrm{e}^{-\beta H_{W+f}^{\mathcal{C}}} m^{\otimes M}(\mathrm{~d} \mathbf{z}) \leqslant M^{2} I_{W}+\frac{1}{8 \pi \beta^{2}}(f,-\Delta f)+\mathrm{O}\left(M^{-A}\right)\|\Delta f\|_{\infty}+\mathrm{O}(M \log M) .
\end{aligned}
$$

Here we have used the improvement commented in the proof of Proposition B.8, which gives the improved factor for the error term proportional to $\|\Delta f\|_{\infty}$ and avoids the restriction on $\|\Delta f\|_{\infty}$ that was assumed in 6 , Lemmas 4.3-4.4]. From this and with $f$ replaced by $f / s$, we obtain the estimate

$$
\frac{1}{\beta} \log \mathbb{E}_{V_{t}}^{G_{t}} \mathrm{e}^{X_{f / s}}=\frac{1}{8 \pi s^{2} \beta^{2}}(f,-\Delta f)+\mathrm{O}\left(M^{-A}\right) \frac{1}{s}\|\Delta f\|_{\infty}+\mathrm{O}(E),
$$

with $E=t M K+M \log M$. As in the proof of [6, Lemmas 4.1], choosing

$$
s=E^{-\frac{1}{2}}\|\nabla f\|_{2}+M^{-A} E^{-1}\|\Delta f\|_{\infty},
$$

this implies

$$
\frac{1}{\beta} \log \mathbb{E}_{V_{t}}^{G_{t}} \mathrm{e}^{X_{f} / s}=\mathrm{O}(E) .
$$

By Markov's inequality, $\mathbb{P}\left(X_{f} \geqslant \mathrm{O}(s E)\right) \leqslant \mathrm{e}^{-E}$, and since the same estimate also holds with $f$ replaced by $-f$, we have

$$
\mathbb{P}\left(X_{f}=\mathrm{O}\left(E^{1 / 2}\|\nabla f\|_{2}+M^{-A}\|\Delta f\|_{\infty}\right)\right) \geqslant 1-2 \mathrm{e}^{-E},
$$

which implies the claim (B.52).

Finally, we note that for the Yukawa gas with $\ell \geqslant M^{2}$ we have $Y^{\ell}(z)+\log |z|=$ constant + $O\left(|z| / M^{2}\right)$ by $(2.2)$. The constant part of the energy does not affect the measure and the error term $O\left(1 / M^{2}\right)$ is uniformly bounded by $O(1)$ when summed over all $M^{2}$ pairs of particles (which we may assume to be at distance of order 1 due to the growth of the external potential) and therefore does not affect the estimate either.

As in the proof of [6, Theorem 1.1], the proof of Proposition B.11 now follows from iterated applications of Proposition B.12 to the conditioned measures associated to increasingly small balls. This induction proceeds almost exactly as in [6, Section 6], with the additional element that, in each step, we improve also the bound $K$ for the conditioned measure. We first give an outline of this induction now. Recall that we write $t=N^{-2 a}$. 
First step. In the first step, using 2.9 , the difference $H-H_{W, N}^{\mathcal{C}}$ is bounded uniformly by

$$
\sum_{j, k: j \neq k}\left|\tilde{G}\left(z_{j}, z_{k}\right)\right| \leqslant t \sum_{j \neq k} \mathrm{e}^{-\left|z_{j}-z_{k}\right|^{2} /\left(2 \theta^{2}\right)} \leqslant t M K
$$

with $M=N$ and $K=N$. From Proposition B.12, we therefore get the high probability estimate

$$
\frac{X_{f}}{N} \prec N^{-A-1}\|\Delta f\|_{\infty}+\left(N^{-a}+N^{-\frac{1}{2}}\right)\|\nabla f\|_{2} .
$$

This estimate proves an effective estimate on the number of particles on scales $N^{-s}$ for $s<$ $1 / 4 \wedge a / 2$, i.e., $\gg N^{1 / 4}+t^{1 / 4}$.

Induction. By induction, supposing we can control particle numbers on the distance scale $N^{-r}$, in Proposition B.12 applied to the conditional measure in a ball of the former scale, we have $M \approx N^{1-2 r}$ and $\alpha=N / M \approx N^{2 r}$. With the range of the perturbation in the interaction given by $\theta=N^{-1 / 2+\sigma}$, it follows that (B.51) holds (see Lemma B.14 below) with

$$
K=\mathrm{O}\left(M \vee N^{2 \sigma}\right) .
$$

Using this estimate, by conditioning exactly as in the proof of [6. Theorem 1.1], for any $f$ whose support has diameter at most $N^{-r}$, we obtain from Proposition B.12 the estimate

$$
\begin{aligned}
\frac{X_{f}}{N} & \prec\left(t \frac{M \vee N^{2 \sigma}}{\alpha N}+\alpha^{-1} N^{-1}\right)\|\Delta f\|_{\infty}+\left(t^{\frac{1}{2}} \frac{M \vee\left(M N^{2 \sigma}\right)^{\frac{1}{2}}}{N}+M^{\frac{1}{2}} N^{-1}\right)\|\nabla f\|_{2} \\
& \prec\left(N^{-2 a-4 r}+N^{-1-2 r}\right)\|\Delta f\|_{\infty}+\left(N^{-a-2 r}+N^{-\frac{1}{2}-r}\right)\|\nabla f\|_{2} .
\end{aligned}
$$

This is an effective estimate on particle numbers on scales $N^{-s}$ for $s<\left(r+\frac{a}{2}\right) \vee\left(\frac{1}{4}+\frac{r}{2}\right)+\varepsilon$, improving the assumed estimate. We remark that, as far as the scales are concerned, this is the same recursion as in the case of the Yukawa gas, with $\delta$ replaced by $a / 2$.

To set up the induction formally, we replace the assumption $\left(\mathrm{A}_{r}\right)$ of $[6]$ by the following one. (Note also that as before we changed the index $t$ from condition $\mathrm{A}_{t}$ from [6] into $\mathrm{A}_{r}$ as, in the current paper, $t$ refers to the argument of the Laplace transform).

Assumption $\left(\mathbf{A}_{r}\right)$. For any bounded $f \in C^{2}(\mathbb{C})$ with supp $\Delta f \subset B_{r}^{\circ} \cap S_{V}$, we have

$$
\frac{X_{f}}{N} \prec\left(N^{-1-2 r}+N^{-2 a-4 r}\right)\|\Delta f\|_{\infty}+\left(N^{-\frac{1}{2}-r}+N^{-a-2 r}\right)\|\nabla f\|_{2} .
$$

As shown above, for $r=0$ this is $(\mathrm{B} .52)$ applied with $M=N$ and $V=W$ and the trivial estimate $K=N$. To prove Proposition B.11, it is enough to prove the next proposition.

Proposition B.13. For arbitrary $\varepsilon>0,\left(A_{r}\right)$ implies $\left(A_{s}\right)$ for any

$$
0 \leqslant r<s \leqslant\left(\frac{1}{4}+\frac{r}{2}\right) \wedge\left(\frac{a}{2}+r\right)-\varepsilon
$$

with the implicit constant in (B.54) depending only on $\varepsilon$.

To prove Proposition B.13, exactly as in [6, Sections 5-6], we condition on the outside of a ball $B_{s}$ on scale $s$ and replace the Coulomb potential of the outside charges with the Coulomb potential of the equilibrium measure. To ensure that the equilibrium measure of the conditional system inside $B_{s}$ does not move much under this replacement, we use 6 , Propositions 3.3 and $3.4]$ and the analogues of [6, Lemmas 6.2 and 6.3], where the input assumption is replaced by our new assumption $\left(\mathrm{A}_{r}\right)$; the lemmas are checked exactly as in the case of the Yukawa gas. The additional required estimate is the bound $K$ on (B.51), which is given by the following lemma. 
Lemma B.14. Assume $\left(A_{r}\right)$. Then, with high probability, uniformly for all configurations of the $M$ charges inside $B_{s}$, the estimate (B.51) holds with

$$
K=\mathrm{O}\left(N^{1-2 r} \vee N \theta^{2}\right) .
$$

In particular, if $B$ is at scale $N^{-r}$ and $\theta=N^{-1 / 2+\sigma}$ then the right-hand side is $\mathrm{O}\left(M \vee N^{2 \sigma}\right)$.

Proof. Recall that the perturbation term in the Hamiltonian is bounded by $\sum_{j \neq k} \mathrm{e}^{-\left|z_{j}-z_{k}\right|^{2} /\left(2 \theta^{2}\right)}$.

We split this term into the three contributions: (1) both particles are inside $B,(2)$ one particle is in $B$ and one outside $B$, and (3) both particles are outside $B$. The contribution (3) with both particles outside $B$ is a constant for the conditioned measure and thus irrelevant for the estimate on the conditioned measure. Contribution (1) is trivially estimated by $M^{2}$. Contribution (2) is bounded by $\mathrm{O}\left(M\left(N \theta^{2}+N^{1-2 r}\right)\right)$ by the local density estimate, with $r$-HP for the configurations outside $B$. This gives the claimed estimate.

Proof of Theorem 2.3. As in the proof of Proposition B.13, we condition on the particles outside a ball $B$ of radius $N^{-s}$ and assume that $f$ is supported in the ball with the same center and half of the radius. However, since $\left(\mathrm{A}_{1 / 2-\sigma}\right)$ has already been proved, by Lemma B.14, we now have the optimal estimate $K=\mathrm{O}\left(N^{2 \sigma}\right)$. The theorem then follows directly from the one-step bound (B.52) on any scale $b$ as in the assumption of the theorem using this bound on $K$, implying that $t K=t \mathrm{O}\left(N^{2 \sigma}\right)=\mathrm{O}(1)$.

B.6. Conditioned versions: proof of Theorems 2.8 2.9. The proofs of the conditioned versions of the local density estimates are analogous to the original (unconditioned) versions. Namely, we prove the unconditioned versions by inductive conditioning on increasing small balls. The assumptions of the conditioned versions are exactly such that the inductive assumption is satisfied for the conditional measure. We omit the details.

\section{Notation index}

\section{Interaction}

$\mathcal{C} \quad 2 \mathrm{~d}$ Coulomb interaction, page 2

$G \quad$ generic two-body interaction, page 1

$\tilde{G} \quad$ generic perturbation of the interaction, page 7

$L_{\omega}^{\nu} \quad$ difference between Yukawa interactions $U^{\ell}$ with ranges $\ell=\omega$ and $\ell=\nu$, page 13

$\mathcal{T}_{G} \quad$ Interaction $G$ averaged over translations of the unit torus, page 42

$U^{\ell} \quad$ periodic Yukawa interaction on $\mathbb{T}$ with range $\ell$, page 6

$U_{\alpha}^{\ell} \quad$ periodic Yukawa interaction on the torus $\mathbb{T}_{\alpha}$ with range $\ell$, page 17

$U_{b}^{\ell} \quad$ periodic Yukawa interaction on $\mathbb{T}^{(b)}$ with range $\ell$, page 15

$\bar{Y} \quad$ interaction $Y_{u}^{\ell}$ averaged over $u$, page 18

$Y^{\ell} \quad$ Yukawa interaction on $\mathbb{C}$ with range $\ell$, page 6

$\tilde{Y}_{u}^{\ell} \quad$ sum of periodic Yukawa interaction on tori $\mathbb{T}_{\alpha}$, with origin $u$, page 17

\section{Potential}

$Q=Q_{R}^{\ell}$ effective potential for the Yukawa gaz with range $\ell$, page 28

$U_{\mu}^{\ell} \quad$ Yukawa potential with range $\ell$ associated to a measure $\mu$, on the torus, page 8

$V \quad$ external potential, for the Coulomb of Yukawa gas, on the plane or torus, page 1

$Y_{\mu}^{\ell} \quad$ Yukawa potential with range $\ell$ associated to a measure $\mu$, on the plane, page 8 


\section{Hamiltonian, energy}

$\hat{A}_{V}^{f} \quad$ local angle term for the test function $f$, page 60

$A_{V}^{h,+} \quad$ long-range angular term, page 63

$A_{V}^{h,-} \quad$ short-range angular term, page 63

$H_{N, V}^{G} \quad$ Hamiltonian for interaction $G$ and external potential $V$, page 1

$H_{N, V}^{\ell} \quad$ Hamiltonian for the Yukawa interaction on $\mathbb{T}$ with range $\ell$ and external potential $V$, page 7

$\hat{H}_{\alpha} \quad$ Hamiltonian for the interaction $U_{\alpha}^{\ell}$, page 17

$\tilde{H}_{u}^{\ell} \quad$ Hamiltonian associated to interaction $\tilde{Y}_{u}^{\ell}$, page 17

$I_{V} \quad$ minimum of $\mathcal{I}_{V}$, page 2

$\mathcal{I}_{V} \quad$ energy functional with Coulomb interation and external potential $V$, page 2

$\mathcal{I}_{V}^{\ell} \quad$ energy functional with Yukawa interaction with range $\ell$ and external potential $V$, page 7

$K_{R}^{\ell} \quad$ equilibrium energy of the Yukawa potential from scale $\ell$ to $R$, page 28

$\mathbf{L}_{\omega}^{\nu} \quad \mathbf{L}_{\omega}^{\nu}=\int L_{\omega}^{\nu}(z-w) \tilde{\mu}(\mathrm{d} w) \tilde{\mu}(\mathrm{d} z)$, page 13

$W_{V}^{G, v} \quad$ evaluated Hamiltonian in the Ward identity, page 61

\section{Measure}

$\mathbb{E}^{A} \quad$ expectation for the Gibbs measure associated to a Hamiltonian $A$, page 12

$m \quad$ Lebesgue measure on $\mathbb{C}$ or on the torus, page 2

$\mu_{V} \quad$ equilibrium measure for external potential $V$ and Coulomb interaction, minimizer of $\mathcal{I}_{V}$, page 2

$\mu_{V}^{\ell} \quad$ equilibrium meaure for external potential $V$ and Yukawa interaction with range $\ell$, minimizer of $\mathcal{I}_{V}^{\ell}$, page 8

$\hat{\mu} \quad$ empirical measure, page 2

$\tilde{\mu} \quad$ difference between empirical measure and equilibrium measure, page 9

$P_{N, V, \beta}^{G} \quad$ Gibbs measure for interaction $G$, external potential $V$, inverse temperature $\beta$, page 2

$\rho_{V} \quad$ density of $\mu_{V}$, page 2

\section{Partition function}

$\xi_{b}^{(\gamma)}(n) \quad$ a normalized version of $\log Z_{b, n}^{(\gamma)}$, page 15

$\xi^{(\ell)}(N) \quad$ a normalized version of $\log Z_{N}^{(\ell)}$, page 12

$\zeta^{(\ell)}(N) \quad$ torus residual free energy, a normalized version of $\log Z_{N}^{(\ell)}$, page 12

$F(\mathbf{n}) \quad$ quasi-free free energy for particle profile $\mathbf{n}$, page 17

$Z_{N}^{(\ell)} \quad$ associated to Hamiltonian $H_{N}^{(\ell)}$, at inverse temperature $\beta$, page 12

$Z_{b, n}^{(\gamma)} \quad$ associated to Hamiltonian with interaction $U_{b}^{\ell}$, at inverse temperature $\beta(\gamma=\ell / b)$, page 15

$Z_{N, V, \beta}^{G} \quad$ associated to Hamiltonian $H_{N, V}^{G}$, at inverse temperature $\beta$, page 2

\section{Other Symbols}

$\alpha \quad$ index of the squares, page 16

$b \quad$ mesoscopic scale for test function, also noted $N^{-s}$, page 3

$b \quad$ torus side length, page 15 


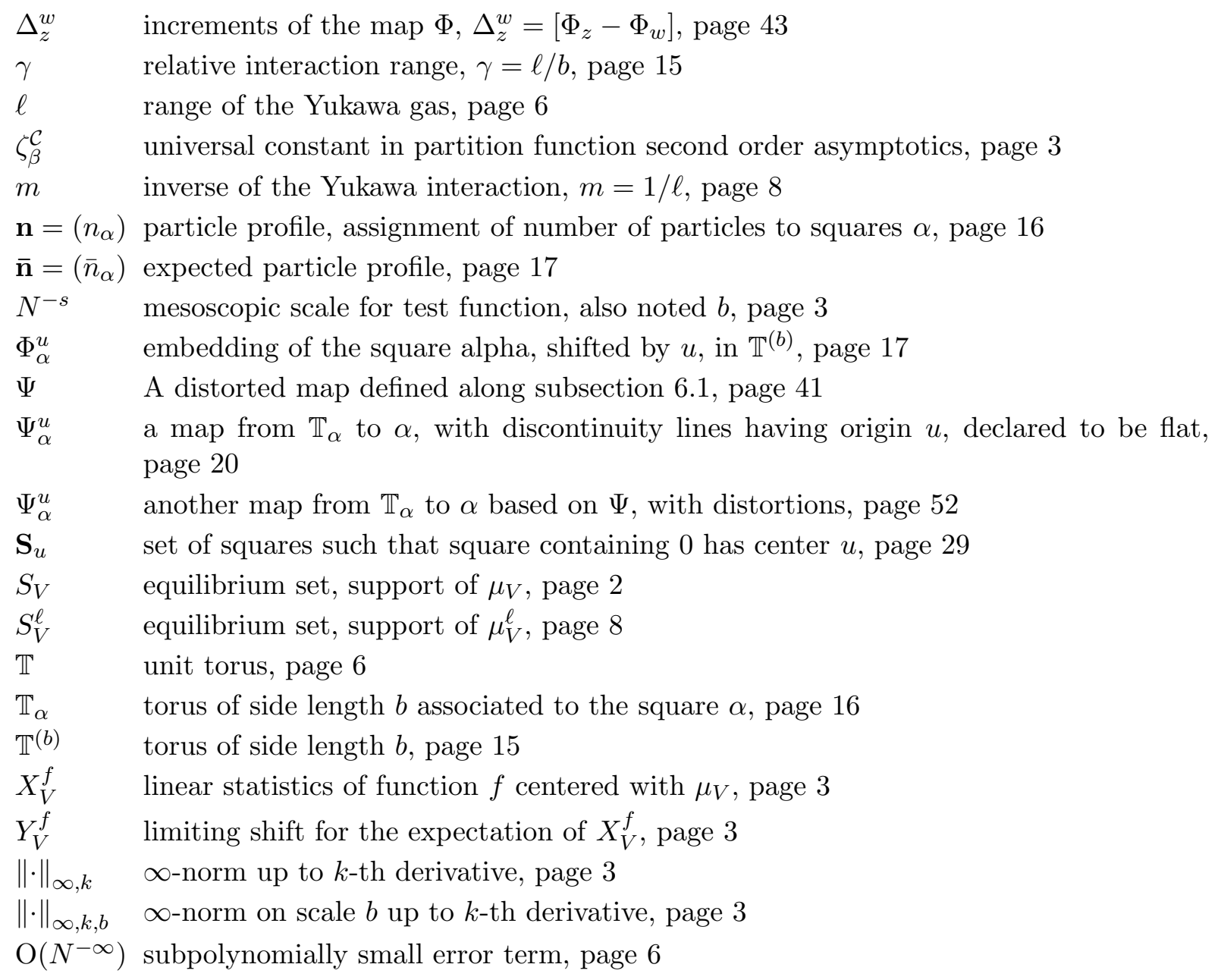

\section{References}

[1] M. Aizenman and P.A. Martin. Structure of Gibbs states of one-dimensional Coulomb systems. Comm. Math. Phys., 78(1):99-116, 1980/81.

[2] A. Alastuey and B. Jancovici. On the classical two-dimensional one-component Coulomb plasma. J. Physique, 42(1):1-12, 1981.

[3] Y. Ameur. A density theorem for weighted Fekete sets. Int. Math. Res. Not. IMRN, (16):5010-5046, 2017.

[4] Y. Ameur, H. Hedenmalm, and N. Makarov. Fluctuations of eigenvalues of random normal matrices. Duke Math. J., 159(1):31-81, 2011.

[5] Y. Ameur, H. Hedenmalm, and N. Makarov. Random normal matrices and Ward identities. Ann. Probab., 43(3):1157-1201, 2015.

[6] R. Bauerschmidt, P. Bourgade, M. Nikula, and H.-T. Yau. Local density for two-dimensional one-component plasma. Comm. Math. Phys., 356(1):189-230, 2017.

[7] F. Bekerman and A. Lodhia. Mesoscopic central limit theorem for general $\beta$-ensembles. Ann. Inst. Henri Poincaré Probab. Stat., 54(4):1917-1938, 2018. 
[8] G. Borot and A. Guionnet. Asymptotic expansion of beta matrix models in the multi-cut regime, 2013. Preprint, arXiv:1303.1045.

[9] G. Borot and A. Guionnet. Asymptotic expansion of $\beta$ matrix models in the one-cut regime. Comm. Math. Phys., 317(2):447-483, 2013.

[10] P. Bourgade, L. Erdös, and H.-T. Yau. Universality of general $\beta$-ensembles. Duke Math. J., 163(6):1127-1190, 2014.

[11] P. Bourgade, L. Erdős, H.-T. Yau, and J. Yin. Fixed energy universality for generalized Wigner matrices. Comm. Pure Appl. Math., 69(10):1815-1881, 2016.

[12] H.J. Brascamp and E.H. Lieb. On Extensions of the Brunn-Minkowski and Prékopa-Leindler Theorems, Including Inequalities for Log Concave Functions, and with an Application to the Diffusion Equation, pages 441-464. Springer Berlin Heidelberg, Berlin, Heidelberg, 2002.

[13] D.C. Brydges and P. Federbush. Debye screening. Comm. Math. Phys., 73(3):197-246, 1980.

[14] D.C. Brydges and P.A. Martin. Coulomb systems at low density: a review. J. Statist. Phys., 96(5-6):1163-1330, 1999.

[15] J.M. Caillol, D. Levesque, J.J. Weis, and J.P. Hansen. A Monte Carlo study of the classical two-dimensional one-component plasma. Journal of Statistical Physics, 28(2):325-349, 1982 .

[16] L.-L. Chau and Y. Yu. Unitary polynomials in normal matrix models and wave functions for the fractional quantum Hall effects. Phys. Lett. A, 167(5-6):452-458, 1992.

[17] J.G. Conlon, E.H. Lieb, and H.-T. Yau. The $N^{7 / 5}$ law for charged bosons. Comm. Math. Phys., 116(3):417-448, 1988.

[18] J.G. Conlon, E.H. Lieb, and H.-T. Yau. The Coulomb gas at low temperature and low density. Comm. Math. Phys., 125(1):153-180, 1989.

[19] B. Eynard. Topological expansion for the 1-Hermitian matrix model correlation functions. J. High Energy Phys., (11):031, 35 pp. (electronic) (2005), 2004.

[20] C. Fefferman and R. de la Llave. Relativistic stability of matter. I. Rev. Mat. Iberoamericana, 2(1-2):119-213, 1986.

[21] P.J. Forrester. Log-gases and random matrices, volume 34 of London Mathematical Society Monographs Series. Princeton University Press, Princeton, NJ, 2010.

[22] J. Ginibre. Statistical ensembles of complex, quaternion, and real matrices. J. Mathematical Phys., 6:440-449, 1965.

[23] D.P. Hardin, E.B. Saff, B.Z. Simanek, and Y. Su. Next order energy asymptotics for Riesz potentials on flat tori. Int. Math. Res. Not. IMRN, (12):3529-3556, 2017.

[24] H. Hedenmalm and N. Makarov. Coulomb gas ensembles and Laplacian growth. Proc. Lond. Math. Soc. (3), 106(4):859-907, 2013.

[25] J.Z. Imbrie. Debye screening for jellium and other Coulomb systems. Comm. Math. Phys., 87(4):515-565, 1982/83. 
[26] B. Jancovici, J.L. Lebowitz, and G. Manificat. Large charge fluctuations in classical Coulomb systems. J. Statist. Phys., 72(3-4):773-787, 1993.

[27] K. Johansson. On Szegö's asymptotic formula for Toeplitz determinants and generalizations. Bull. Sci. Math. (2), 112(3):257-304, 1988.

[28] K. Johansson. On fluctuations of eigenvalues of random Hermitian matrices. Duke Math. J., 91(1):151-204, 1998.

[29] N.-G. Kang and N.G. Makarov. Gaussian free field and conformal field theory. Astérisque, (353):viii+136, 2013.

[30] H. Kunz. The one-dimensional classical electron gas. Ann. Phys., 85(2):303-335, 1974.

[31] R.B. Laughlin. Anomalous quantum hall effect: An incompressible quantum fluid with fractionally charged excitations. Phys. Rev. Lett., 50:1395-1398, May 1983.

[32] T. Leblé. Local microscopic behavior for 2D Coulomb gases. Probab. Theory Related Fields, 169(3-4):931-976, 2017.

[33] T. Leblé and S. Serfaty. Large deviation principle for empirical fields of log and Riesz gases. Invent. Math., 210(3):645-757, 2017.

[34] T. Leblé and S. Serfaty. Fluctuations of two dimensional Coulomb gases. Geom. Funct. Anal., 28(2):443-508, 2018.

[35] E.H. Lieb and J.L. Lebowitz. The constitution of matter: Existence of thermodynamics for systems composed of electrons and nuclei. Advances in Math., 9:316-398, 1972.

[36] E.H. Lieb, N. Rougerie, and J. Yngvason. Rigidity of the Laughlin liquid. J. Stat. Phys., $172(2): 544-554,2018$.

[37] E.H. Lieb, N. Rougerie, and J. Yngvason. Local incompressibility estimates for the Laughlin phase. Comm. Math. Phys., 365(2):431-470, 2019.

[38] M. Petrache and S. Rota Nodari. Equidistribution of jellium energy for Coulomb and Riesz interactions. Constr. Approx., 47(1):163-210, 2018.

[39] B. Rider. Deviations from the circular law. Probab. Theory Related Fields, 130(3):337-367, 2004.

[40] B. Rider and B. Virág. The noise in the circular law and the Gaussian free field. Int. Math. Res. Not. IMRN, (2):Art. ID rnm006, 33, 2007.

[41] N. Rougerie and S. Serfaty. Higher-dimensional Coulomb gases and renormalized energy functionals. Comm. Pure Appl. Math., 69(3):519-605, 2016.

[42] N. Rougerie and J. Yngvason. Incompressibility estimates for the Laughlin phase. Comm. Math. Phys., 336(3):1109-1140, 2015.

[43] E.B. Saff and V. Totik. Logarithmic potentials with external fields, volume 316 of Grundlehren der Mathematischen Wissenschaften [Fundamental Principles of Mathematical Sciences]. Springer-Verlag, Berlin, 1997. Appendix B by Thomas Bloom. 
[44] E. Sandier and S. Serfaty. 2D Coulomb gases and the renormalized energy. Ann. Probab., 43(4):2026-2083, 2015.

[45] S. Serfaty. Coulomb gases and Ginzburg-Landau vortices. Zurich Lectures in Advanced Mathematics. European Mathematical Society (EMS), Zürich, 2015.

[46] M. Shcherbina. Fluctuations of linear eigenvalue statistics of $\beta$ matrix models in the multicut regime. J. Stat. Phys., 151(6):1004-1034, 2013.

[47] A. Soshnikov. The central limit theorem for local linear statistics in classical compact groups and related combinatorial identities. Ann. Probab., 28(3):1353-1370, 2000.

[48] P. Wiegmann and A. Zabrodin. Large scale correlations in normal non-Hermitian matrix ensembles. J. Phys. A, 36(12):3411-3424, 2003. Random matrix theory.

[49] A. Zabrodin. Random matrices and Laplacian growth. In The Oxford handbook of random matrix theory, pages 802-823. Oxford Univ. Press, Oxford, 2011.

[50] A. Zabrodin and P. Wiegmann. Large- $N$ expansion for the 2D Dyson gas. J. Phys. A, 39(28):8933-8963, 2006. 